Michell Macedo Alves

\title{
MÉTODO DA PARTIÇÃo NA ANÁLISE DE MÚLTIPLAS FISSURAS
}

Tese apresentada à Escola de Engenharia de São Carlos, Universidade de São Paulo, como parte dos requisitos para obtenção do título de Doutor em Engenharia de Estruturas.

Orientador: Prof. Tit. Sergio Persival Baroncini Proença

São Carlos - SP

2010 
AUTORIZO A REPRODUÇÃO E DIVULGAÇÃO TOTAL OU PARCIAL DESTE TRABALHO, POR QUALQUER MEIO CONVENCIONAL OU ELETRÔNICO, PARA FINS DE ESTUDO E PESQUISA, DESDE QUE CITADA A FONTE.

Ficha catalográfica preparada pela Seção de Tratamento da Informação do Serviço de Biblioteca - EESC/USP

\footnotetext{
A474mes, Michell Macedo
Método da partição na análise de múltiplas fissuras / Michell Macedo Alves ; orientador Sergio Persival Baroncini Proença. -- São Carlos, 2010.

Tese (Doutorado-Programa de Pós-Graduação e Área de Concentração em Engenharia de Estruturas) -- Escola de Engenharia de São Carlos da Universidade de São Paulo, 2010 .

1. Método sem malha. 2. Método dos elementos finitos. 3. Método da partição. 4. Mecânica da fratura. 5. Fator de intensidade de tensão. I. Título.
} 
FOLHA DE JULGAMENTO

Candidato: Engenheiro MICHELL MACEDO ALVES.

Tese defendida e julgada em 03/09/2010 perante a Comissão Julgadora:

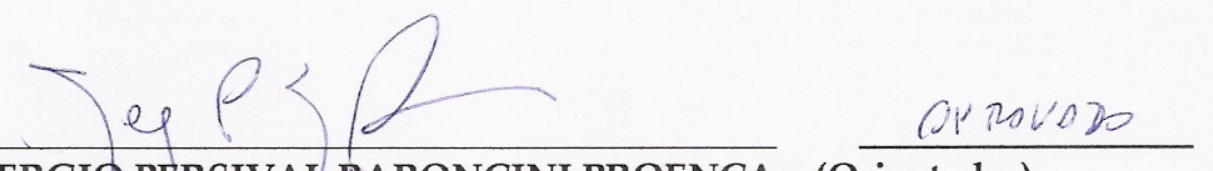

Prof. Titular SERGIO PERSIVAL BARONCINI PROENÇA - (Orientador) (Escola de Engenharịa de São Cartọs/USP)

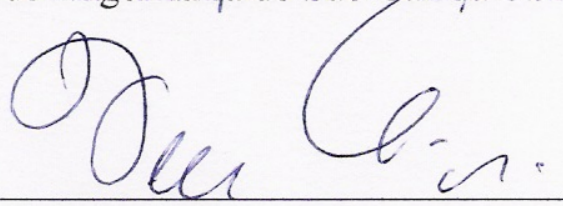

ARPO TADO

Prof. Dr. WALTER LIBARDI

(Universidade Federal de São Carlos/UFSCar)

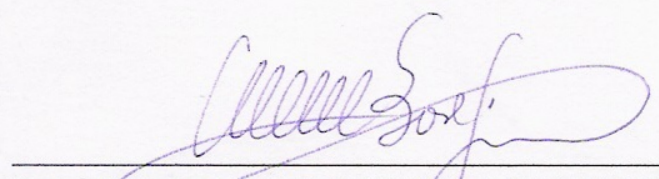

ApPOVADO.

Prof. Titular WALDEK WLADIMIR BOSE FILHO (Escola de Engenharia de São Carlos/USP)

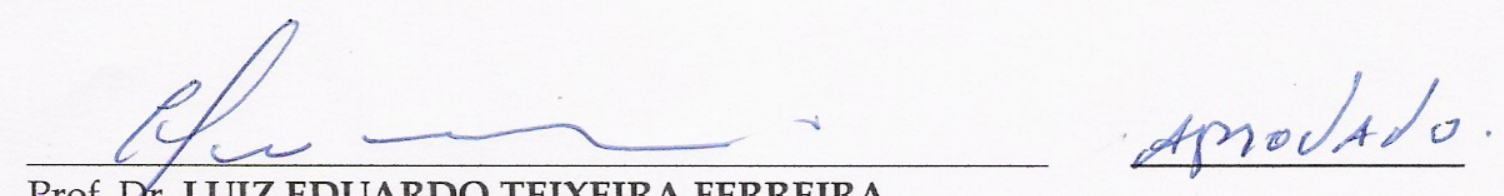

Prof. Dr. LUIZ EDUARDO TEIXEIRA FERREIRA

(Centro Universitário das Faculdades de Ensino FAE de São João da Boa Vista/UNIFAE)

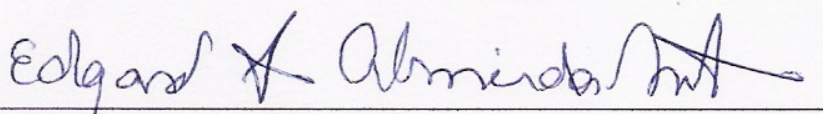

Aprovado

Prof. Dr. EDGARD SANT'ANNA DE ALMEIDA NETO (Escola Politécnica/USP)

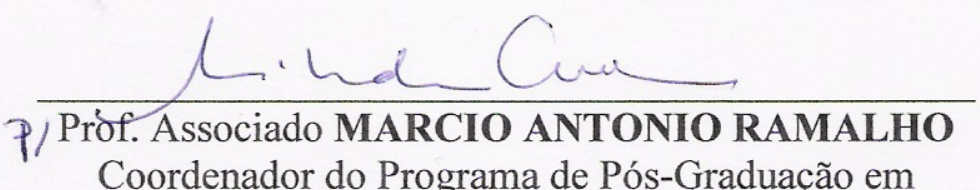

Coordenador do Programa de Pós-Graduação em Engenharia Civil (Engenharia de Estruturas)

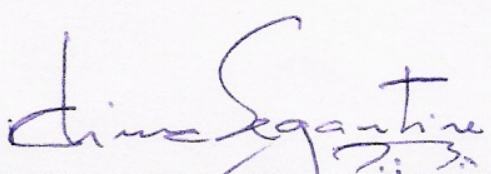

Prof. Associado GERALDO ROBERTO MARTINS DA COSTA

Presidente da Comissão de Pós-Graduação

Prof. Associado Pauto César Lima Segantine

Vice-Presidente da CPG 
Dedico este trabalho a toda minha família e ao meu Mestre Aloizio de Assis Paniago (In Memoriam), um homem cujo amor, a paciência e a bondade mudaram minha vida. 
“Combati o bom combate,

Percorri o meu caminho, Guardei a fé."

Paulo - II Timóteo 4:7 


\section{AGRADECIMENTOS}

Primeiramente a Deus por ter me dado esta oportunidade de crescimento assim como a força necessária para que eu chegasse ao fim da jornada.

À Escola de Engenharia de São Carlos pela oportunidade e por todo o conhecimento que me foi concedido.

À Capes pela bolsa de estudo concedida.

Ao Professor Sergio Persival Baroncini Proença, pela maravilhosa orientação que sempre se fez de forma paciente e atenciosa. Agradeço também pela amizade criada nestes últimos anos, assim como pelo constante incentivo em vencer os obstáculos do caminho. Aos professores Jorge Munaiar e José Samuel Giongo pelo companheirismo e amizade. Ao Professor Felício Bruzzi Barros pela imensa ajuda e boa vontade no auxílio em parte da pesquisa.

Aos funcionários do Departamento de Estruturas: Rosi, Nadir, Toninho, Eli, Silvia e Paccola pelos auxílios prestados que sempre vieram de forma muito gentil.

Aos colegas do Departamento de Estruturas: Marlos Mangini, Dorival Piedade, Edmar Theóphilo, Ronaldo Rigobello, Walter Oliveira, Wesley Góis, Eduardo Toledo, Edson Leonel, Saulo José, Manoel Dênis, Raimundo Amorim, Érica Kimura e Higor Argolo pelos auxílios prestados e principalmente pela amizade.

Aos colegas de República: Robson Caldo, Thiago Galante, Davi Santos, Marcos Bêdo, Daniel Ferreira, Rodrigo Girckus, William Jandoza, Uziel Paulo e Rafaela Fernandes pelo auxílio e amizade criados neste período de convivência.

À Dona Júlia, Dona Eva e Sr. Donizetti pela amizade e por todo o auxílio prestado, tendo me acolhido como um filho em São Carlos.

Aos "mais que amigos" e "mais que irmãos" Marcelo Manzato e Carolina Ferraz que iluminaram meu caminho nos momentos difíceis e trouxeram muitas alegrias neste período de convivência em que juntos lutamos e servimos ao Cristo.

Aos meus familiares: meus pais, meus irmãos, meu vovô Chico, vovó Dilma, vovó Luzia, aos primos Cacá, Marussa, Norton e Sueli, aos tios Carlinhos, Maria Lúcia, Norma, Fátima, Jerlos, Júnia, Abadia, Valério e Jerominho e ao nosso amigo do mundo invisível "Zé Uberaba" que foram meus alicerces e que me apoiaram em cada instante para que eu chegasse 
até a vitória. Agradeço também à benção constante de nossa Senhora Aparecida em todos os momentos desta jornada assim como as bençãos do nosso querido amigo Meishu-Sama.

Aos irmãos da Associação Espírita "Francisco Thiesen" e Posto de Assistência "Irmão Áureo" pela convivência, amizade e aprendizado. O bem que plantamos nestes últimos anos permanecerá vivo no meu coração. E aos novos irmãos da Associação Espírita "Chico Xavier" pela amizade, em especial aos meus grandes amigos Onaldo Campos e Yara Souza. A todos que direta ou indiretamente contribuíram para realização desta pesquisa. 


\section{RESUMO}

ALVES, M. M. (2010). Método da Partição na Análise de Múltiplas Fissuras. Tese (Doutorado) - Escola de Engenharia de São Carlos, Universidade de São Paulo, São Carlos.

Neste trabalho apresenta-se a formulação do problema de múltiplas fissuras baseada numa abordagem de superposição utilizada pelo Método da Partição (Splitting Method). Um dos objetivos principais deste trabalho refere-se à aferição da capacidade deste método na obtenção de fatores de intensidade de tensão, tendo em vista o seu desenvolvimento recente e a ausência de outras fontes de pesquisa além daquelas oriundas dos seus próprios autores. Segundo a abordagem do Método da Partição, os fatores de intensidade de tensão finais de uma estrutura podem ser encontrados a partir da sobreposição de três subproblemas. Deste modo, o problema é resolvido mediante imposição de que nas faces das fissuras as tensões que resultam da sobreposição sejam nulas. Sendo assim, apresenta-se a formulação do Método da Partição para uma ou mais fissuras e diversas análises numéricas que contemplam interação entre fissuras submetidas aos modos I e II de abertura. Outra etapa do trabalho refere-se à aplicação do Método dos Elementos Finitos Generalizados (MEFG) num dos subproblemas, dito local, ao invés do emprego do Método dos Elementos Finitos (MEF), que em sua forma convencional pode requerer um refinamento excessivo da malha, particularmente junto à ponta da fissura, aumentando o custo computacional da análise. Exemplos de simulação numérica são apresentados no sentido de comprovar que a utilização do MEFG viabiliza a obtenção de resultados com boa aproximação mesmo com malhas pouco refinadas, reduzindo significativamente o custo computacional de toda a análise. Além disto, é apresentada a formulação do Método da Partição para casos que contemplam também fissuras internas, uma vez que a formulação atual admite somente fissuras de borda.

Palavras-chave: Método dos Elementos Finitos Generalizados, Método da Partição, Mecânica da Fratura, Fator de Intensidade de Tensão. 


\section{ABSTRACT}

ALVES, M. M. (2010). Splitting Method in the Analysis of Multi-Site Cracks. PhD Thesis School of Engineering of São Carlos, University of São Paulo, São Carlos.

This work presents the formulation of the problem of multiple cracks based on an superposition approach used by the Splitting Method. The main goal of this work concerns the verification of the ability of this method of obtaining stress intensity factors, in view of its recent development and the absence of other research sources beyond those derived from their own authors. According to the approach of Splitting Method, the final stress intensity factors of a structure can be found from the superposition of three subproblems. Thus, the problem is solved by superposition and then imposing the nullity of the stresses on the faces of cracks. Thus, the formulation of the Splitting Method is presented to one or more cracks and also several numerical simulations that consider the interaction between cracks subjected to opening mode I and II. Another part of this work concerns the application of the Generalized Finite Element Method (GFEM) in the local subproblem instead of the use of Finite Element Method (FEM), which in its conventional form may require an excessive mesh refinement, particularly near the tip the crack, increasing the computational cost of analysis. Examples of numerical simulation are presented in order to show that the use of GFEM enables to obtain results with good approximation even with little refined meshes, thus significantly reducing the computational cost of the entire analysis. Moreover, the formulation of the Splitting Method is presented for cases which also have internal cracks due to the current formulation admits only boundary cracks.

Keywords: Generalized Finite Element Method, Splitting Method, Fracture Mechanics, Stress Intensity Factor. 


\section{LISTA DE FIGURAS}

Figura 2.1 - Navio Liberty que sofreu ruptura frágil.........................................................6

Figura 2.2 - Navio Liberty partido em dois..................................................................

Figura 2.3 - Plataforma A. L. Keilland após o acidente......................................................

Figura 2.4 - A. L. Keilland. Suporte da base quebrado devido à ruptura na solda.....................8

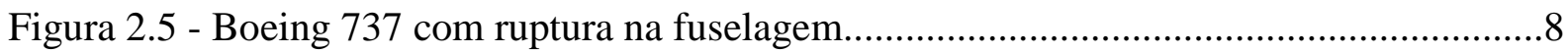

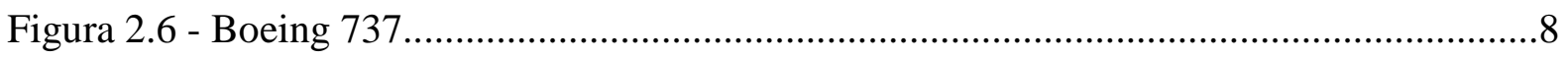

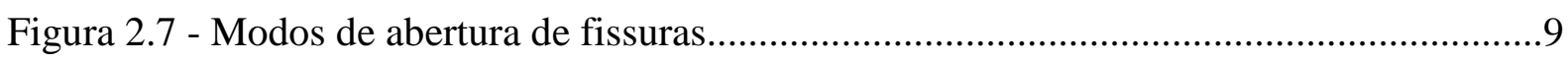

Figura 2.8 - Chapa de tamanho infinito submetida a carregamento biaxial............................11

Figura 2.9 - Sistema de coordenadas do campo de tensões e deslocamentos...........................11

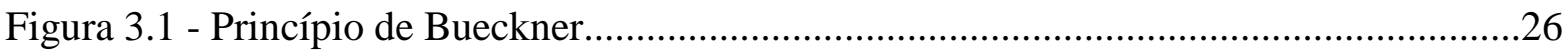

Figura 3.2 - Carregamento na face de uma fissura resolvido com o Princípio

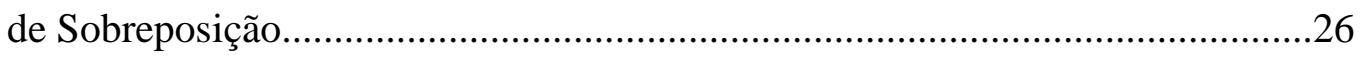

Figura 3.3 - Princípio de Sobreposição e Princípio de Bueckner............................................28

Figura 3.4 - Caso clássico da Mecânica da Fratura dividido em dois subproblemas...............28

Figura 3.5 - Método da Partição para uma fissura baseado no Princípio de

Sobreposição e Princípio de Bueckner.

Figura 3.6 - Método da Partição baseado na extrapolação do Princípio de

Sobreposição e Princípio de Bueckner para duas fissuras..................................29

Figura 3.7 - Divisão do segundo subproblema do Princípio de Sobreposição..........................30

Figura 3.8 - Exemplo de um sólido com múltiplas fissuras....................................................31

Figura 3.9 - Subproblemas segundo o Método da Partição.....................................................32

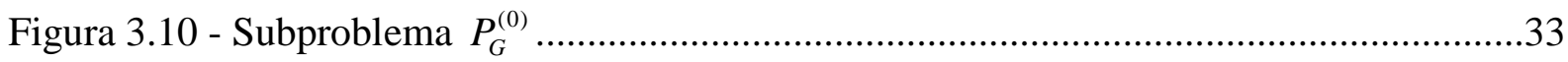




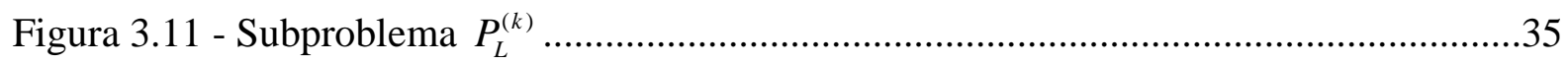

Figura 3.12 - Valores de K calculados em $10 \%$ da face da fissura........................................38

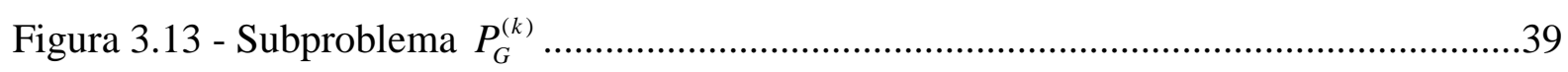

Figura 4.1 - Chapa com 1 fissura e carregamento constante..............................................50

Figura 4.2 - Chapa com 1 fissura e carregamentos variados................................................53

Figura 4.3 - Eixo das coordenadas locais e pontos de coleta das tensões................................55

Figura 4.4 - Chapa com 2 fissuras e carregamento cte+quadrático........................................57

Figura 4.5 - Definição do contorno $\Gamma^{(i)}$ para as linhas de fissura........................................59

Figura 4.6 - Fluxo de tensões $\mathrm{Q}_{\mathrm{j}}$ aplicado nas faces da fissura para obtenção de $\mathrm{K}_{\mathrm{II}} \ldots \ldots \ldots \ldots \ldots . . . . .60$

Figura 4.7 - Chapa com 1 fissura e carregamento cte+quadrático cisalhante..........................61

Figura 4.8 - Chapa com 2 fissuras e carregamento constante unitário cisalhante.....................62

Figura 4.9 - Variação das tensões de cisalhamento nas linhas de fissura

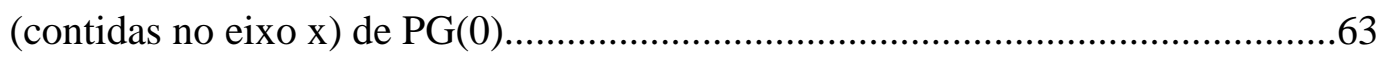

Figura 4.10 - Chapa com 2 fissuras e carregamento cte+quadrático cisalhante......................64

Figura 4.11 - Exemplo de formatação de força nodal aplicada em linguagem LOGFILE......66

Figura 4.12 - Campo “ANSYS Command Prompt”.........................................................66

Figura 4.13 - Exemplo de formatação de deslocamento nodal imposto em linguagem

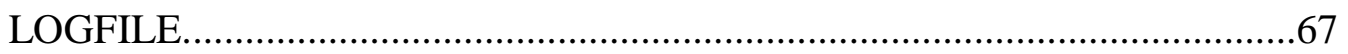

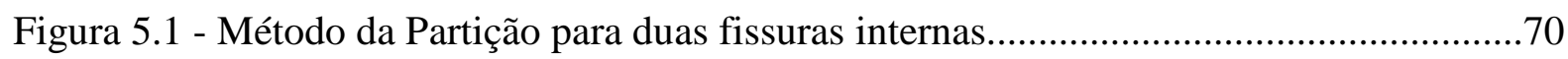

Figura 5.2 - Sistemas de Coordenadas Locais para fissuras internas...................................71

Figura 5.3 - Influências da vizinhança do contorno $\Gamma^{(i)}$ na determinação

dos fatores de intensidade de tensão............................................................. 72

Figura 5.4 - Carregamentos aproximativos Q para fissuras internas.....................................73

Figura 5.5 - Chapa com 1 fissura e carregamento constante................................................87

Figura 5.6 - Subdivisão do problema original pelo Método da Partição.................................88 
Figura 5.7 - Chapa com 1 fissura e carregamento cte+quadrático.........................................90

Figura 5.8 - Pontos utilizados para a coleta de valores de tensão.........................................91

Figura 5.9 - Chapa com 2 fissuras e carregamento cte+quadrático.....................................92

Figura 5.10 - Chapa com 2 fissuras e carregamento cisalhante constante..............................94

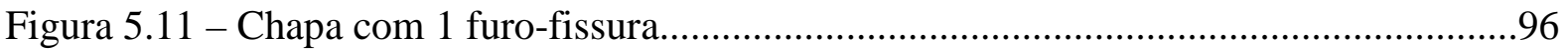

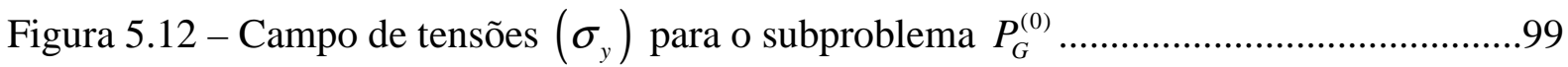

Figura 5.13 - Campo de tensões $\left(\sigma_{y}\right)$ na ponta da fissura - Subproblema $P_{L}^{(k=1)} \ldots \ldots \ldots \ldots \ldots \ldots . . . .99$

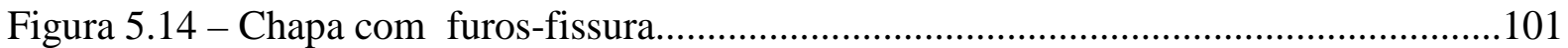

Figura 5.15 - Delimitação do domínio local de $P_{L}^{(k)}$...........................................................102

Figura 5.16 - Chapa com 3 fissuras internas e 2 fissuras de borda com carregamento constante

Figura 5.17 - 1/4 da chapa devido à utilização dos eixos de simetria horizontal e vertical....105

Figura 5.18 - Chapa com 2 fissuras de borda, sendo uma delas na vizinhança de um furo...110

Figura 6.1 - Nuvens de influência dos nós A e B de uma malha regular de elementos finitos quadrilaterais

Figura 6.2 - Enriquecimento da partição da Unidade num domínio global bidimensional....118 Figura 6.3 - Elemento Finito Quadrilateral de 4 nós Regular no

domínio paramétrico e Distorcido no domínio físico.

Figura 6.4 - Orientação dos eixos locais da fissura.

Figura 6.5 - Chapa Contendo Uma Fissura em Modo I

de Abertura - Carregamento Constante. 136

Figura 6.6 - Malha Refinada para o Caso de Uma Fissura..................................................136

Figura 6.7 - Detalhe do Refinamento na Ponta da Fissura.................................................137

Figura 6.8 - Malha Graduada para o Caso de Uma Fissura................................................137

Figura 6.9 - Chapa Contendo Duas Fissuras em Modo I 
de Abertura - Carregamento Constante.........................................................139

Figura 6.10 - Malha Refinada para o Caso de Duas Fissuras...............................................139

Figura 6.11 - Malha Graduada para o Caso de Duas Fissuras............................................140

Figura 6.12 - Detalhe da Malha Graduada na Região da Ponta de Fissura - Modo I.............140

Figura 6.13 - Chapa Contendo Duas Fissuras em Modo I

de Abertura - Carregamento Constante....................................................... 141

Figura 6.14 - Dimensões do Subproblema Local - Modo I de Abertura...............................142

Figura 6.15 - Chapa Contendo Duas Fissuras em Modo I

de Abertura - Carregamento Cte+Quadrático.

Figura 6.16 - Chapa Contendo Duas Fissuras em Modo

II de Abertura - Carregamento Constante. .145

Figura 6.17 - Dimensões do Subproblema Local - Modo II de Abertura..............................146

Figura 6.18 - Malha Refinada para o Subproblema Local - Modo II...................................146

Figura 6.19 - Malha Graduada para o Subproblema Local - Modo II..................................147

Figura 6.20 - Detalhe da Malha Graduada na Região da Ponta de Fissura - Modo II............147

Figura B.1 - Condição de Cauchy-Riemann......................................................................167 


\section{LISTA DE SÍMBOLOS}

a) Letras Romanas Maiúsculas

[B] - Matriz que relaciona deslocamentos nodais com deformações nodais da estrutura

$C$ - Constante dependente das características geométricas da chapa, é utilizada para a determinação do valor do fator de intensidade de tensão

COD - "Crack Open Displacement" a diferença entre os deslocamentos de pontos correspondentes das faces segundo direção perpendicular à linha de fissura.

CSD - "Crack Sliding Displacement" a diferença entre os deslocamentos de pontos correspondentes das faces segundo direção paralela à linha de fissura.

$E(\%)$ - Erro em porcentagem

E - Módulo de Elasticidade Longitudinal (ou de Young)

$\mathfrak{I}_{n}^{p}$ - Família de funções utilizadas pela base de enriquecimento para $n$ número de nós e grau polinomial $p$

$\{F\}$ - Vetor de forças nodais da estrutura

$G$ - Módulo de Elasticidade Transversal (ou de cisalhamento)

H - Altura de uma chapa (sólido bidimensional)

I - Número de fissuras

$\operatorname{Im}[Z]$ - Parte imaginária da função complexa Z

[IG] - Matriz de "influência geral" reunindo os termos que definem a interação entre as fissuras

$[I G]_{L}$ - Matriz que possui as integrais responsáveis por representar as tensões em $P_{L}^{(k)}$ 
$[I G]_{G}$ - Matriz que possui as integrais responsáveis pelo efeito de interação entre as fissuras $\left(P_{G}^{(k)}\right)$

J - Número de termos da aproximação utilizados pelo Método da Partição

K - Fator de Intensidade de Tensão

$\mathrm{K}_{\mathrm{I}}$ - Fator de Intensidade de Tensão para o Modo I de Abertura

$\mathrm{K}_{\mathrm{II}}$ - Fator de Intensidade de Tensão para o Modo II de Abertura

$\mathrm{K}_{\mathrm{III}}$ - Fator de Intensidade de Tensão para o Modo III de Abertura

$\mathrm{K}_{\mathrm{A}}$ - Fator de Intensidade de Tensão para o Subproblema A

$K^{(i)}(J)$ - Fator de Intensidade de tensão da fissura $i$ obtido pelo Método da Partição com a utilização de $\mathrm{J}$ termos de aproximação da base polinomial

$K_{j}^{(i)}$ - Fator de Intensidade de Tensão da fissura $i$ obtido mediante utilização do termo de aproximação $j$ (carregamento aproximativo aplicado nas faces da fissura $i$ )

$K_{R E F}$ - Fator de Intensidade de Tensão de Referência (ou exato para alguns casos)

$K_{A P R O X}$ - Fator de Intensidade de Tensão Aproximado

$[\mathrm{K}]$ - Matriz de rigidez da estrutura

$L_{\alpha, \beta}($ fiss $)$ - Espaço das funções para a base aproximativa do campo de tensões de uma dada fissura, dependente das variáveis $\alpha$ e $\beta$

M - Valor máximo do índice $k$ utilizado pelo Método da Partição na sobreposição dos subproblemas

$P_{G}^{(0)}$ - Subproblema Global Zero do Método da Partição

$P_{L}^{(k)}$ - Subproblema Local $k$ do Método da Partição

$P_{G}^{(k)}$ - Subproblema Global $k$ do Método da Partição

$P_{G}$ - Problema Original 
$Q_{j}\left(\xi_{i} / a_{i}\right)$ - carregamento aproximativo constituído por uma base de $j$ polinômios

$Q_{1}^{(1)}, Q_{1}^{(2)}$ - Constantes pertencentes às funções enriquecedoras da fratura para os modos I e II de abertura respectivamente.

$R_{j}\left(\xi_{i} / a_{i}\right)$ - carregamento aproximativo constituído por uma base de $j$ funções racionais

$\operatorname{Re}[Z]$ - Parte real da função complexa $Z$

$S$ - Linha de contorno do domínio de um sólido

$S_{i}$ - Superfície do vazio da fissura $i$

$S_{c i}$ - Superfície da fissura $i$ composta por partes $S_{c i}^{+}, S_{c i}^{-}$representando as faces superior e inferior respectivamente

$S_{t}$ - Superfície de um sólido com forças prescritas

$S_{u}$ - Superfície de um sólido com deslocamentos prescritos

$S_{0}^{(i)}$ - Linha de contorno do domínio local $V_{L}^{(i)}$ (reproduzindo condições de meio infinito)

$\underline{\underline{T}}$ - Tensor de Tensão para EPT ou EPD

$V$ - Domínio de um sólido sem a presença de fissuras para os subproblemas $P_{G}^{(0)}$ e $P_{G}^{(k)}$

$V_{L}^{(i)}$ - Domínio local com a presença de fissura para o subproblema $P_{L}^{(k)}$

W - Largura de uma chapa (sólido bidimensional)

$Z_{I}$ - Função de tensão de Westergaard representativa da distribuição de tensão no problema de fratura para o modo I de abertura

$Z_{I I}$ - Função de tensão de Westergaard representativa da distribuição de tensão no problema de fratura para o modo II de abertura

$Z_{I I I}$ - Função de tensão de Westergaard representativa da distribuição de tensão no problema de fratura para o modo III de abertura 
b) Letras Romanas Minúsculas

$a_{i}$ - Comprimento da fissura i (medido segundo sua projeção em relação a um eixo local $\xi_{i}$ )

$b_{i, j}$ - coeficientes das combinações lineares das tensões do subproblema $P_{G}^{(0)}$ referentes à fissura $i$ e termo de aproximação $j$

$c_{i, j}^{(k)}$ - coeficientes das combinações lineares das tensões do subproblema $P_{G}^{(k)}$ referentes à fissura $i$, termo de aproximação $j$ e subproblema $k$

$f_{j}^{i}$ - Função enriquecedora número $i$ para a direção $j$

$n$ - Normal à linha de contorno de um dado sólido bidimensional

$\underline{n}$ - Vetor normal à linha de contorno de um dado sólido bidimensional

$n f u i$ - Número de funções utilizadas para o enriquecimento na direção $u$ referente ao nó i

$n f v i$ - Número de funções utilizadas para o enriquecimento na direção $v$ referente ao nó i

$p u_{i}^{j}$ - Parâmetro adicional resultante da estratégia de enriquecimento do nó $i$ na direção $u$ pela função número $j$

$p v_{i}^{j}$ - Parâmetro adicional resultante da estratégia de enriquecimento do nó $i$ na direção $v$ pela função número $j$

$r$ - raio (coordenada cilíndrica com origem na ponta da fissura)

$r$ - No item 3.6.2 - Distância da ponta da fissura até o ponto considerado para a coleta de COD ou CSD (na configuração indeformada)

r - Nas figuras $6.5,6.9$ e 6.14 - raio com origem na ponta da fissura que engloba os nós enriquecidos 
$\{r\}$ - Vetor pertencente à formulação do Método da Partição para fissuras de borda que contêm as integrais com valores de tensões referentes ao subproblema $P_{G}^{(0)}$

$\left\{r l^{(i)}\right\}$ - Sub-Vetor pertencente à formulação do Método da Partição para fissuras internas que contêm as integrais com valores de tensões referentes à ponta de fissura $i$ do subproblema $P_{G}^{(0)}$

$t$ - Espessura do elemento finito isoparamétrico

$\underline{t}$ - Vetor de tensão imposto no contorno com normal $n$

$t_{j}^{(i)}$ - Campo de tensões do contorno ${ }^{(i)} \Gamma$ que envolve a fissura $i$ submetida a um carregamento $Q_{j}$ aplicado em suas faces

$t_{G}^{(0)}$ - Vetor contendo os valores de tensões das linhas de fissura do subproblema $P_{G}^{(0)}$

$\underline{t}_{L}^{(k)}$ - Vetor contendo os valores de tensões das linhas de fissura do subproblema $P_{L}^{(k)}$

$t_{G}^{(k)}$ - Vetor contendo os valores de tensões das linhas de fissura do subproblema $P_{G}^{(k)}$

$u$ - Deslocamento horizontal (eixo $x$ )

$\underline{u}$ - Vetor de deslocamento imposto no contorno $S$ de um dado sólido

$u_{j}^{(i)}$ - Campo de deslocamentos do contorno ${ }^{(i)} \Gamma$ que envolve a fissura $i$ submetida a um carregamento $Q_{j}$ aplicado em suas faces

$u_{i}$ - Deslocamento do nó $i$ do elemento finito isoparamétrico na direção $u$

$u_{x^{\prime}}^{(I)}(r, \theta), u_{y^{\prime}}^{(I)}(r, \theta)$ - Solução analítica do campo de deslocamentos nas direções $x^{\prime}$ e $y^{\prime}$ respectivamente, na forma de uma série infinita para problemas da mecânica da fratura em modo $I$ de abertura

$u_{x^{\prime}}^{(I I)}(r, \theta), u_{y^{\prime}}^{(I I)}(r, \theta)$ - Solução analítica do campo de deslocamentos nas direções $x^{\prime}$ e $y^{\prime}$ respectivamente, na forma de uma série infinita para problemas da mecânica da fratura em modo II de abertura 
$v$ - Deslocamento vertical (eixo $y$ )

$v_{i}$ - Deslocamento do nó $i$ do elemento finito isoparamétrico na direção $v$

$w$ - Deslocamento na direção espessura de um sólido bidimensional (eixo z)

z - No cap. 2 - distância do ponto médio de uma fissura interna até outro ponto qualquer

c) Letras Grega Maiúscula

${ }^{(i)} \Gamma$ - Contorno arbitrário envolvendo a fissura $i$

$\Omega_{i}$ - No Cap. 2 - Equação harmônica $i \quad(i=0,1,2)$

$\Omega$ - No Cap. 6 - Domínio da estrutura

d) Letras Gregas Minúsculas

$\alpha_{k}$ ou $\{\alpha\}$ - Fatores de escala $\alpha$ do Método da Partição empregados na determinação dos fatores de intensidade de tensão para cada fissura

$\alpha_{R}$ - Parâmetro pertencente à função que atende ao problema contendo o conjunto furofissura

$\beta_{R}$ - Parâmetro pertencente à função que atende ao problema contendo o conjunto furofissura

$\varepsilon_{x}, \varepsilon_{y}, \gamma_{x y}$ - Componentes planas das deformações

$\theta$ - Coordenada cilíndrica angular 
$\theta$ - No Cap. 6 - Ângulo entre o segmento da fissura (eixo $X^{\prime}$ - fig. 6.4) e a reta formada pelo segmento com origem na ponta da fissura e extremidade em um ponto qualquer

$\theta_{G}$ - No Cap. 6 - Ângulo entre o eixo global X (vide fig. 6.4) e a reta formada pelo segmento com origem na ponta da fissura e extremidade em um ponto qualquer

$\theta_{0}$ - No Cap. 6 - Ângulo entre o eixo global X (vide fig. 6.4) e o eixo $\mathrm{X}^{\prime}$, formado pelo segmento da fissura

$\kappa$ - Constante que leva em conta o tipo de deformação da estrutura. É igual a (3-4 v) se Estado Plano de Deformação e $\frac{3-v}{1+v}$ se Estado Plano de Tensão.

$v$ - Coeficiente de Poisson

$\xi$ - No Cap. 2 e Anexos - Raio com origem na ponta de fissura

$\xi_{i}, \eta_{i}, \varsigma_{i}$ - Sistema cartesiano de coordenadas locais atrelado à linha de fissura e com origem na sua ponta, representando as direções longitudinal, normal e transversal ao comprimento da fissura

$\xi, \eta$ - Coordenadas adimensionais do elemento finito isoparamétrico

$\sigma_{x}, \sigma_{y}, \tau_{x y}$ - Componentes planas das tensões

$\sigma(x)$ - Tensão normal aplicada nas faces de uma dada chapa

$\tau(x)$ - Cisalhamento aplicado nas faces de uma dada chapa

$\phi_{j}(x)$ - Funções de forma do Método dos Elementos Finitos $(M E F)$ referentes aos nós $j=1, \ldots, n$

$\psi$ - Função tensão de Airy para a solução de problemas da elasticidade bidimensional $\omega_{j}$ - região de influência de uma nuvem sobre o nó $j$ (vide Método das Nuvens “ $h p$ ”) 
xxiv

e) Símbolos Matemáticos

$\nabla^{2}$ - Operador Harmônico de Laplace

f) Alfa-Numéricos

1as pontas - Índice que indica as primeiras pontas de fissuras internas envolvidas por um dado contorno ${ }^{(i)} \Gamma$ (Uma vez que cada contorno ${ }^{(i)} \Gamma$ envolve duas pontas de fissura, ou seja, uma fissura interna)

2as pontas - Índice que indica as segundas pontas de fissuras internas envolvidas por um dado contorno ${ }^{(i)} \Gamma$ (Uma vez que cada contorno ${ }^{(i)} \Gamma$ envolve duas pontas de fissura, ou seja, uma fissura interna) 


\section{LISTA DE TABELAS}

Tabela 2.1 - Expressões dos Deslocamentos em Função de Z para Modos I e II de abertura EPT e EPD 18

Tabela 2.2 - Expressões Deslocamento x Tensões em Função de $r$ e $\theta$, para Modos

I e II de abertura - EPT e EPD. .20

Tabela 4.1 - Valores-Referência de $\mathrm{K}_{\mathrm{I}}$ para carregamentos variados 54

Tabela 4.2 - Valores de $\mathrm{K}_{\mathrm{I}}$ aproximado obtidos para carregamento linear. 55

Tabela 4.3 - Valores de $\mathrm{K}_{\mathrm{I}}$ aproximado obtidos para carregamento quadrático. .55

Tabela 4.4 - Valores de $\mathrm{K}_{\mathrm{I}}$ aproximado obtidos para carregamento cte+quadrático. 56

Tabela 4.5 - Valores-Referência de $\mathrm{K}_{\mathrm{I}}$ para carregamento cte+quadrático 58

Tabela 4.6 - Valores de $\mathrm{K}_{\mathrm{I}}$ aproximados obtidos para carregamento cte+quadrático (2 fissuras) 58

Tabela 4.7 - Valores de $\mathrm{K}_{\mathrm{II}}$ aproximado obtidos para carregamento cte+quadr. Cisalhante....61

Tabela 4.8 - Valores-Referência de $\mathrm{K}_{\text {II }}$ para carregamento constante.

Tabela 4.9 - Valores de $\mathrm{K}_{\mathrm{II}}$ aproximados obtidos para carregamento constante (2 fissuras)

Tabela 4.10 - Valores de $\mathrm{K}_{\mathrm{II}}$ aproximados obtidos para carregamento

Cte+quadrático (2 fissuras) 64

Tabela 5.1 - Valores de $\mathrm{K}_{\mathrm{I}}$ exatos obtidos para carregamento cte+quadrático. .90

Tabela 5.2 - Valores de $\mathrm{K}_{\mathrm{I}}$ aproximado obtidos para carregamento cte+quadrático.

Tabela 5.3 - Valores-Referência de $\mathrm{K}_{\mathrm{I}}$ exatos para 2 fissuras internas e carregamento cte+quadrático

Tabela 5.4 - Valores de $\mathrm{K}_{\mathrm{I}}$ aproximados obtidos para 2 fissuras internas e carregamento cte+quadrático 
Tabela 5.5 - Valores-Referência de $\mathrm{K}_{\text {II }}$ para carregamento cisalhante Constante. .94

Tabela 5.6 - Valores de $\mathrm{K}_{\mathrm{II}}$ aproximados obtidos para carregamento

cisalhante constante. .95

Tabela 5.7 - Valor de K para o exemplo contendo 1 furo-fissura. 97

Tabela 5.8 - Valores-Referência de K obtidos para o exemplo contendo 1 furo-fissura........100

Tabela 5.9 - Valores de K para o exemplo com 2 furos-fissura. 102

Tabela 5.10 - Valores-Referência de K para o exemplo contendo 2 furos-fissura. 103

Tabela 5.11 - Valores-Referência de $\mathrm{K}_{\mathrm{I}}$ para chapa contendo 1 fissura interna e 2 de borda para carregamento constante. 106

Tabela 5.12 - Erro da aproximação para os valores de $\mathrm{K}_{\mathrm{I}}$ 109

Tabela 5.13 - Valores de $\mathrm{K}_{\mathrm{I}}$ para chapa com fissura de borda e borda-furo. 110

Tabela 5.14 - Valores de K obtidos para o exemplo contendo fissura de borda-furo e fissura de borda simples.

Tabela 6.1 - Valores de $\mathrm{K}_{\mathrm{I}}$ aproximados obtidos - via MEFG - Problema

Original com 1 fissura. 138

Tabela 6.2 - Valores de $\mathrm{K}_{\mathrm{I}}$ aproximados obtidos - via MEFG -

Problema Original com 2 fissuras 140

Tabela 6.3 - Valores de $\mathrm{K}_{\mathrm{I}}$ aproximados obtidos - via MEFG - Método da Partição com 2 fissuras

Tabela 6.4 - Valores de $\mathrm{K}_{\mathrm{I}}$ aproximados obtidos - via MEFG -

Subproblemas Locais. .144

Tabela 6.5 - Valores de $\mathrm{K}_{\mathrm{I}}$ aproximados obtidos - via MEFG - Método da Partição com 2 fissuras. 145 
Tabela 6.6 - Valores de $\mathrm{K}_{\mathrm{II}}$ aproximados obtidos - via MEFG - Método da

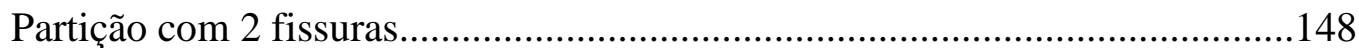

Tabela C.1 - Comparativo entre as Funções-Tensão de Westergaard e as Tensões................175 


\section{ABREVIATURAS}

COD - Crack Open Displacement

CSD - "Crack Sliding Displacement"

Cte - Constante

EPD - Estado Plano de Deformação

EPT - Estado Plano de Tensão

MEF - Método dos Elementos Finitos

MEFG - Método dos Elementos Finitos Generalizados

MEFPU - Método dos Elementos Finitos Partição da Unidade

MMQM - Método dos Mínimos Quadrados Móveis

MP - Método da Partição

NGL - Número de Graus de Liberdade

PU - Partição da Unidade

PVC - Problema de Valor de Contorno 


\section{SUMÁRIO}

RESUMO..................................................................................................................................

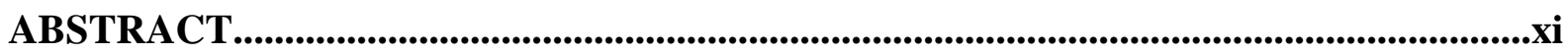

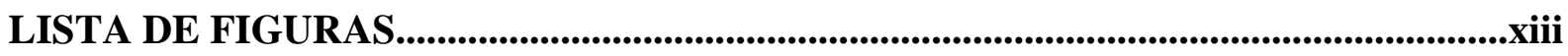

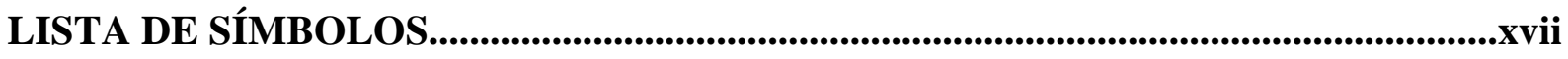

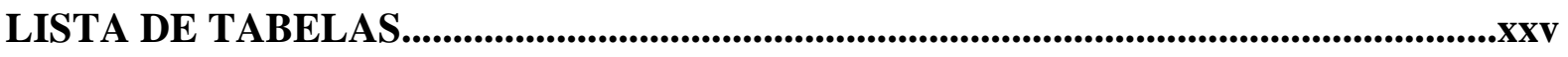

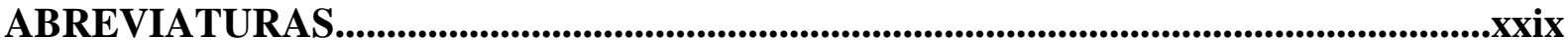

1 INTRODUÇÃ

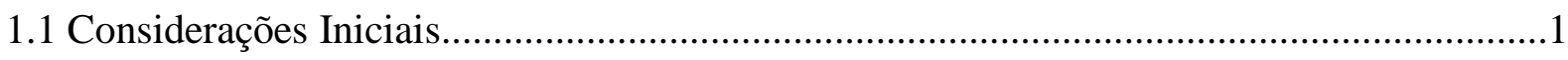

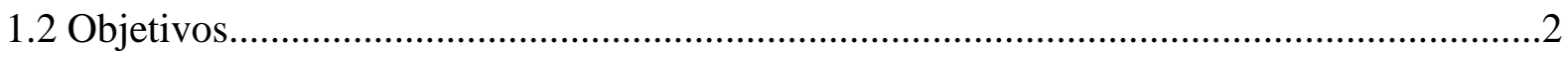

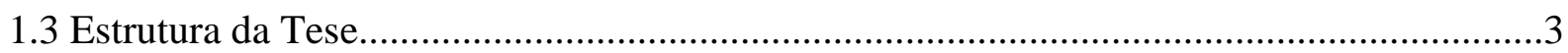

2 MECÂNICA DA FRATURA ELÁSTICO-LINEAR...........................................................5

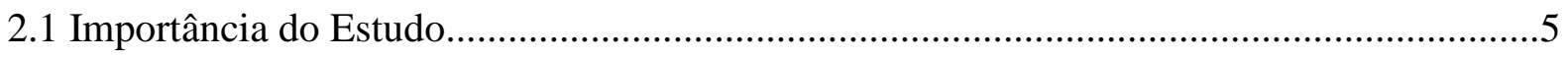

2.2 Mecânica da Fratura: Conceitos Básicos....................................................................

2.2.1 Função Tensão de Airy e as Funções Complexas de Westergaard na Resolução

de Problemas da Mecânica da Fratura Elástico-Linear..................................................10

2.2.2 Equações das Tensões em Função de uma Variável Complexa - Modos

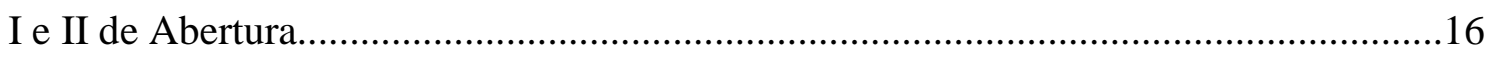

2.2.3 Componentes de Deslocamento em Função de uma Variável Complexa -

Modos I e II de Abertura - EPT e EPD. 
2.2.4 Relações entre os Campos de Tensão e Deslocamento na Vizinhança

da Ponta da Fissura - Modos I e II de Abertura.

2.2.5 Equações que Definem os Campos de Tensão e Deslocamento próximos

à Ponta da Fissura - Modo III de Abertura....................................................................20

2.2.6 O Fator de Intensidade de Tensão

\section{MÉTODO DA PARTIÇÃO NA ANÁLISE DE PROBLEMAS COM MÚLTIPLAS}

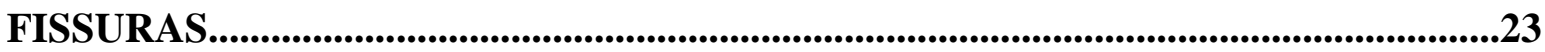

3.1 Breve Histórico Sobre as Análises de Múltiplas Fissuras...............................................23

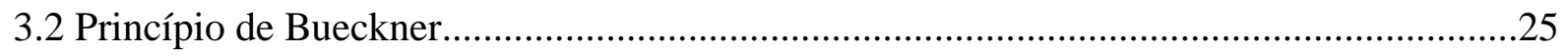

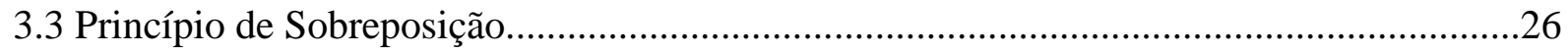

3.4 Princípio de Sobreposição e de Bueckner como Ferramentas do Método da Partição.......27

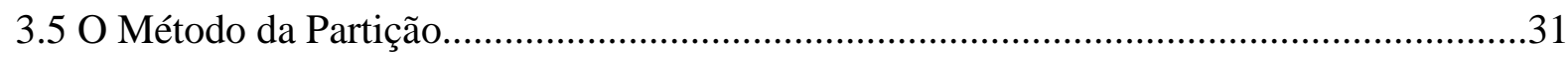

3.6 Os Subproblemas do Método da Partição..........................................................................33

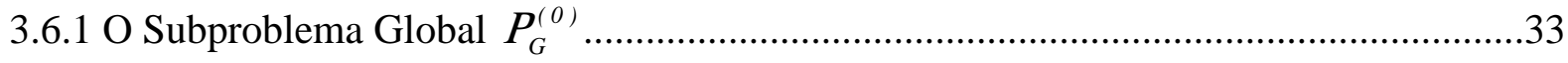

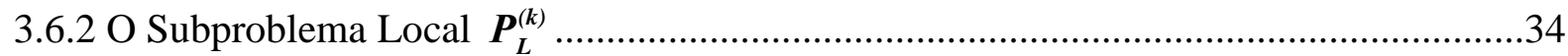

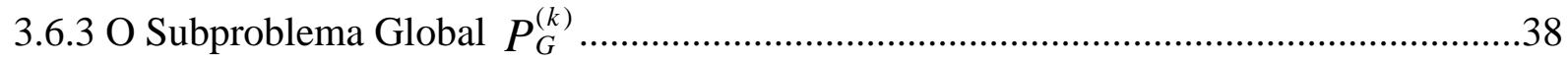

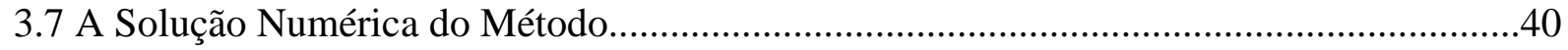

3.7.1 O Sistema Linear $[I G] \cdot\{\alpha\}=\{r\}$ para Uma Fissura...........................................42

3.7.2 O Sistema Linear $[I G] \cdot\{\alpha\}=\{r\}$ Para Várias Fissuras..........................................43

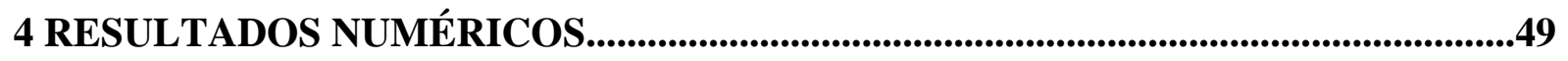

4.1 Chapas Contendo Fissuras de Borda Solicitadas ao Modo I de Abertura.........................49

4.1.1 Exemplo 1: Chapa com uma Fissura - Carregamento Uniformemente Distribuído.......49 
4.1.2 Exemplo 2: Chapa com uma Fissura - Carregamentos Não-Uniformes 53

4.1.3 Exemplo 3: Chapa com Duas Fissuras - Carregamento Não-Uniforme .56

4.2 Chapas Contendo Fissuras de Borda Solicitadas ao Modo II de Abertura .60

4.2.1 Exemplo 1: Chapa com Uma Fissura - Carregamento Não-Uniforme .60

4.2.2 Exemplo 2: Chapa com Duas Fissuras - Carregamento Uniformemente Distribuído.....62

4.2.3 Exemplo 3: Chapa com Duas Fissuras - Carregamento Não-Uniforme .64

4.3 Resolução dos Casos do Método da Partição Utilizando o Programa

ANSYS e a Linguagem de Programação Fortran

\section{COMBINAÇÃO DE FISSURAS INTERNAS, DE BORDA E FISSURA-FURO EM} MODO I DE ABERTURA. 69

5.1 A Formulação Estendida do Método para Casos que Contemplam Fissuras Internas........70

5.1.1 A Solução Numérica para Casos Envolvendo Fissuras Internas .74

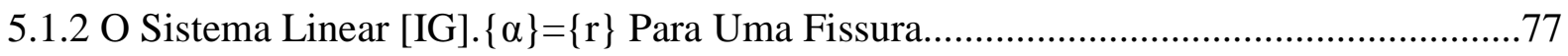

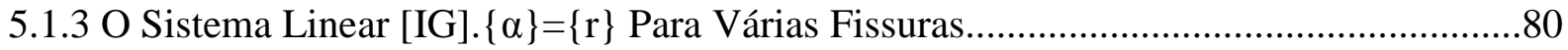

5.1.4 Exemplo 1: Chapa com Uma Fissura - Carregamento Uniformemente Distribuído.......86

5.1.5 Exemplo 2: Chapa com Uma Fissura - Carregamento Não-Uniforme............................90

5.1.6 Exemplo 4: Chapa com Duas Fissuras - Carregamento Não-Uniforme..........................92

5.1.8 Exemplo 6: Chapa com Duas Fissuras - Carregamento Uniformemente Distribuído.....94 5.2 O Emprego de Funções Racionais na Aproximação de Casos Contendo Fissuras-Furo....96

5.2.1 Exemplo 1: Chapa com Uma Fissura-Furo - Carregamento

Uniformemente Distribuído - Modo I de Abertura .96

5.2.2 Exemplo 2: Chapa com Duas Fissuras-Furo - Carregamento

Uniformemente Distribuído - Modo I de Abertura 100

5.3 Combinação de casos envolvendo fissura interna,

de borda e borda-fissura 104 
5.3.1 Exemplo 1: Chapa Contendo Fissuras Internas e de Borda - Carregamento

Uniformemente Distribuído .104

5.3.2 Exemplo 2: Chapa Contendo Fissura de Borda e Fissura-Furo

- Carregamento Uniformemente Distribuído

\section{MÉTODO DOS ELEMENTOS FINITOS GENERALIZADOS NA ANÁLISE DO SUBPROBLEMA LOCAL} 113

6.1 Problemas Envolvendo Singularidades e a Estratégia de Enriquecimento... 113

6.2 Breve Histórico Sobre o MEFG. 114

6.2 A Formulação do Método dos Elementos Finitos Generalizados. 116

6.3 O Elemento Finito Isoparamétrico Enriquecido.

6.4 A Solução Analítica da Fratura como Função Enriquecedora do

Subproblema Local do Método da Partição. 126

6.5 Exemplos Numéricos 135

6.5.1 O Enriquecimento do Problema Original - Modo I de Abertura. 135

6.5.2 Método da Partição com Enriquecimento do Subproblema Local - Modo I de Abertura .141

6.5.3 Método da Partição com Enriquecimento do Subproblema Local - Modo II de abertura. 


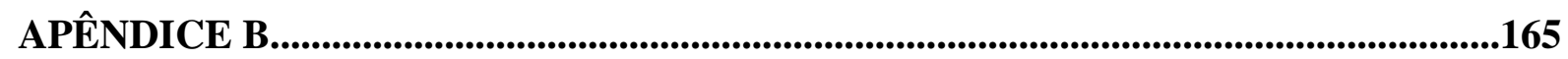

APÊNDICE C.....................................................................................................................................169

APÊNDICE D.........................................................................................................179

APÊNDICE E...................................................................................................................183

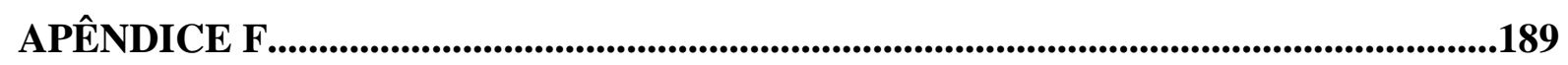




\section{Introdução}

\subsection{Considerações Iniciais}

O presente trabalho trata da aplicação e desenvolvimento do Método da Partição (Splitting Method), de Babuška e Andersson (2005) na análise bidimensional plana de sólidos elástico-lineares contendo múltiplas fissuras. Tal Método que possui suas bases fundamentadas nos Princípios de Sobreposição e de Bueckner, propõe uma decomposição do problema original em três subproblemas que sobrepostos permitem a determinação dos fatores de intensidade de tensão finais do problema original, ou mesmo, campos de tensão e deslocamento, a partir da condição de que nas faces das fissuras os fluxos de tensão resultantes sejam nulos.

Todos os subproblemas do Método da Partição podem ser resolvidos numericamente através do Método dos Elementos Finitos (MEF) em sua formulação clássica. Todavia, quando se realiza a análise de um dos subproblemas, dito local, que leva em conta o efeito local de concentração de tensão na vizinhança da ponta da fissura, o MEF exige elevado grau 
de refinamento da malha, aumentando o custo computacional. Desta maneira, neste trabalho, propõe-se que o Método dos Elementos Finitos Generalizados (MEFG), Duarte, Babuška e Oden (2000), seja utilizado como alternativa de resolução do subproblema local, tendo-se em vista sua capacidade de proporcionar resultados satisfatórios mesmo com malhas pouco refinadas, reduzindo o custo computacional. Tal desempenho do MEFG decorre do recurso do enriquecimento seletivo da aproximação, particularmente das funções Partição da Unidade (PU) atreladas aos nós cujas 'nuvens' contenham a ponta da fissura. $\mathrm{O}$ enriquecimento pode ser realizado mediante funções que melhor possam representar os campos de deslocamento e de tensão naquela região.

Outro aspecto importante deste trabalho refere-se à extensão do Método da Partição para a consideração de fissuras internas, uma vez que a formulação atual admite somente fissuras de borda. Desta maneira, viabilizando uma análise mais diversificada de um componente estrutural no que diz respeito à presença de múltiplas fissuras.

\subsection{Objetivos}

O desenvolvimento de metodologias de abordagem de problemas da Mecânica da Fratura que ofereçam resultados precisos e com baixo custo computacional vem sendo tema de muitos trabalhos publicados nos últimos anos, Owen e Fawkes (1983); Edwalds e Wanhill (1986); Alliabadi e Rooke (1991); Wang e Atluri (1996); Duarte, Babuska e Oden (2000) dentre outros. A maior parte das abordagens faz uso do MEF, que possui uma ampla experimentação e validação de resultados. Todavia, para determinadas aplicações como os problemas de múltiplas fissuras, o custo computacional do MEF se torna demasiadamente alto ou mesmo inviável. Neste contexto, um dos objetivos deste trabalho consiste em desenvolver uma avaliação crítica do procedimento de análise de múltiplas fissuras proposto pelo Método da Partição, que pela sua característica de divisão do problema mais complexo em subproblemas mais simples.

Uma contribuição dada neste trabalho para a formulação do Método da Partição, originalmente restrita somente às fissuras de borda, é a sua extensão para o caso de fissuras internas, possibilitando a análise de um componente estrutural com fissuras de diversas características geométricas e de posicionamento. 
Entre os subproblemas do Método da Partição destaca-se o subproblema local que tem por objetivo principal a determinação dos fatores de intensidade de tensão. Neste subproblema os elevados gradientes de tensão exigem uma discretização bastante refinada da malha de elementos finitos, sobretudo para a boa representação dos campos de tensão nas vizinhanças da singularidade. Com o objetivo de minorar o custo computacional e o refinamento excessivo da malha de elementos finitos desta etapa, propõe-se neste trabalho a utilização de formulações não-convencionais do MEF, mais precisamente o Método dos Elementos Finitos Generalizados (MEFG). Desta maneira, acredita-se que o presente trabalho contribui significativamente para a viabilização de uma metodologia eficiente para o estudo do problema de múltiplas fissuras, sem a necessidade de refinamento excessivo de malha em nenhuma etapa, além de poder ser aplicado à combinação de fissuras com diversas características geométricas.

\subsection{Estrutura da Tese}

No capítulo 2, apresenta-se uma breve revisão dos conceitos fundamentais da Mecânica da Fratura. Em particular destaca-se a dedução do fator de intensidade de tensão, uma vez que este desenvolvimento em sua forma completa e detalhada não possui muitas fontes na literatura atual.

O capítulo 3 faz-se um breve histórico sobre a evolução dos métodos de análise de múltiplas fissuras. Na seqüência são apresentados os princípios básicos que dão suporte ao Método da Partição, assim como a sua formulação e abordagem dos subproblemas que o compõem.

Exemplos de simulação numérica são apresentados no capítulo 4 com o objetivo de demonstrar a eficiência do método na obtenção de fatores de intensidade de tensão. Sendo apresentados vários casos com diferentes tipos de carregamentos tanto no modo I quanto no modo II de abertura.

O capítulo 5 trata da extensão da formulação do Método da Partição para a consideração de fissuras internas, sendo apresentados, também, exemplos numéricos que comprovam a eficiência desta análise nos modos I e II de abertura. Em seguida descreve-se a formulação mais específica para casos que contemplam fissuras de borda originadas em regiões que contenham furos. A avaliação da capacidade das funções racionais empregadas na 
aproximação dos campos de tensão em problemas desta natureza é objeto de estudo. Posteriormente, descrevem-se os procedimentos necessários para análise de casos que contemplam fissuras internas, de borda e fissura-borda. Exemplos desta natureza são apresentados neste capítulo.

O capítulo 6 trata da técnica de enriquecimento do MEFG para elementos finitos quadrilaterais isoparamétricos e apresenta as funções utilizadas para o enriquecimento da aproximação em problemas da Mecânica da Fratura. Neste capítulo são apresentados exemplos de casos resolvidos nos capítulos anteriores com o Método da Partição, porém com os subproblemas locais tratados com o MEFG. Comparações em relação ao MEF convencional são realizadas com o objetivo de demonstrar a eficácia do MEFG em se obter resultados satisfatórios com malhas não tão refinadas.

O capítulo 7 refere-se às considerações finais, conclusões e propostas para trabalhos futuros. 


\section{Mecânica da Fratura Elástico-Linear}

\subsection{Importância do Estudo}

Anteriormente ao uso dos critérios vindos da Mecânica da Fratura, na maioria dos problemas em engenharia, a resistência de um determinado componente estrutural era definida como sendo o valor de sua tensão de escoamento minorada por um coeficiente de segurança. Os principais fatores que justificam a adoção deste parâmetro referiam-se à existência de imperfeições macroscópicas, dentre outros. Mas, os estudos de Inglis (1913), Griffith (1924), Westergaard (1939) e Irwin (1957) mostraram que a presença de agentes concentradores de tensões (micro-fissuras, cantos, etc) poderiam influenciar a resistência de um elemento estrutural de maneira considerável. Sendo assim, muitos componentes estruturais passaram a ser projetados não somente considerando a tensão crítica, mas também 
controlando características geométricas que favorecem a concentração de tensão e uma limitação sobre o comprimento máximo de micro-fissurações.

As pesquisas sobre fissuras como agentes concentradores de tensões iniciaram-se na década de vinte com os trabalhos de Griffith, os quais permaneceram sem frutos durante alguns anos até que na década de cinqüenta foram lançadas as bases da Mecânica da Fratura. Uma década depois esta ciência passava a ser aplicada extensivamente na indústria de materiais de alta resistência (aeronáutica) e na indústria de aço para projetos de reservatórios de pressão, mais especificamente aqueles utilizados em centrais nucleares de produção de energia elétrica, plataformas de petróleo, etc. (Branco, Fernandes e Castro (1999)).

A presença de fissuras como fator preponderante, no que diz respeito à ruptura de grandes estruturas, foi evidenciada em grandes catástrofes que ocorreram no último século. Dentre estas se encontram:

a) Navios Liberty: entre os anos de 1939 e 1945, durante a segunda Guerra Mundial, 2708 navios Liberty foram construídos e 1031 sofreram rupturas frágeis ou se acidentaram devido à propagação de fissuras. Mais de 200 navios apresentaram rupturas sem qualquer esperança de reparo e em 7 navios, a propagação das fissuras partiu os navios ao meio. Estes acidentes foram os maiores e mais caros experimentos em série que ocorreram ao longo do século e praticamente marcaram o nascimento da Mecânica da Fratura.

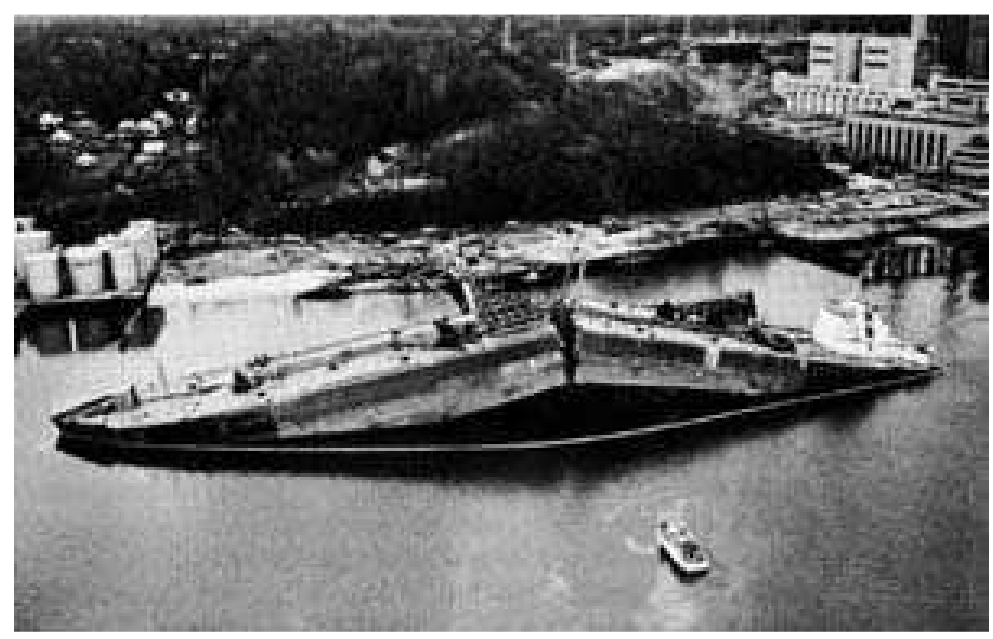

Figura 2.1: Navio Liberty que sofreu ruptura frágil 


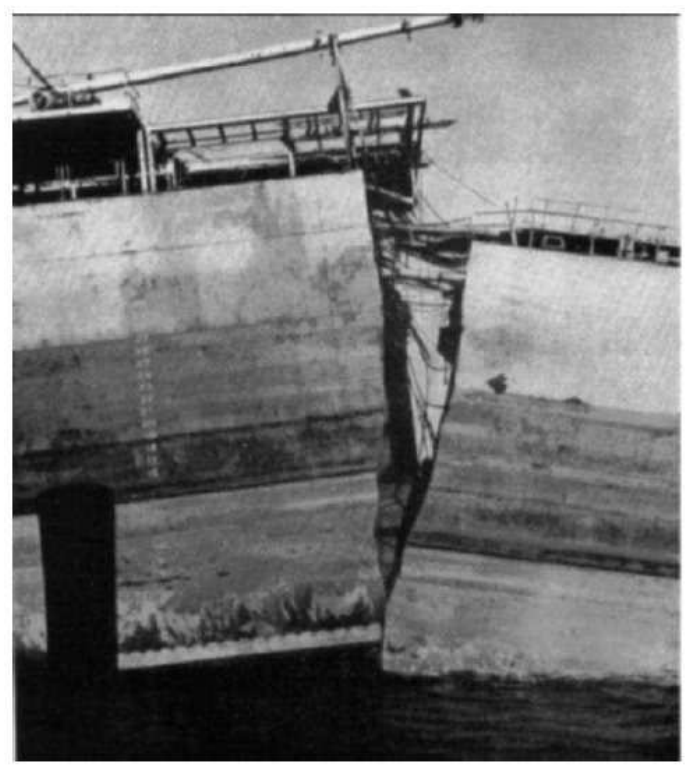

Figura 2.2: Navio Liberty partido em dois

b) Em Março de 1980 afunda a plataforma A. L. Keilland no mar da Noruega devido a uma propagação de fissura oriunda da solda em um componente de sua base. Neste acidente 123 pessoas perderem suas vidas.

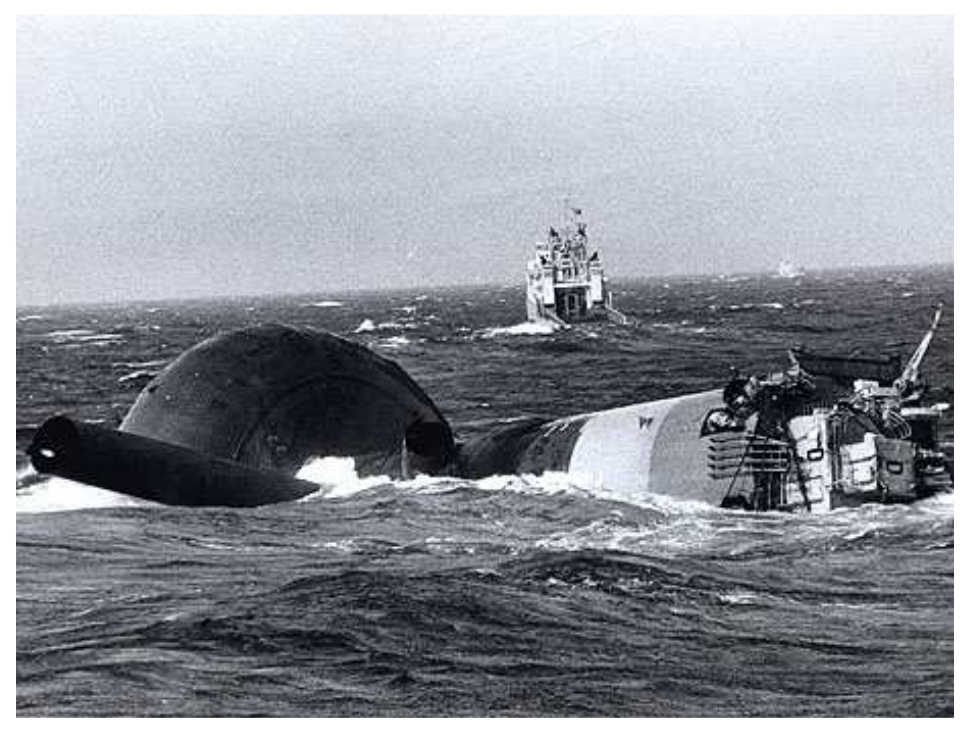

Figura 2.3: Plataforma A. L. Keilland após o acidente 


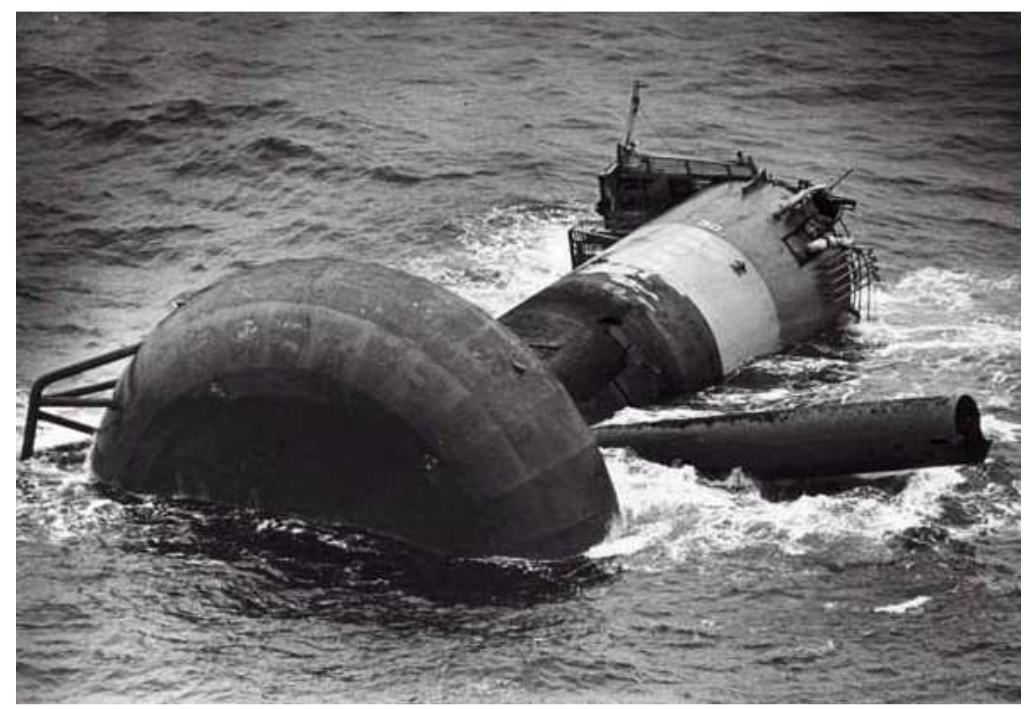

Figura 2.4: A. L. Keilland. Suporte da base quebrado devido à ruptura na solda

c) Em 28 de Abril de 1988 parte da fuselagem de um Boeing se rompeu após 19 anos de serviço. A ruptura foi causada por fadiga e a presença de múltiplas fissuras.

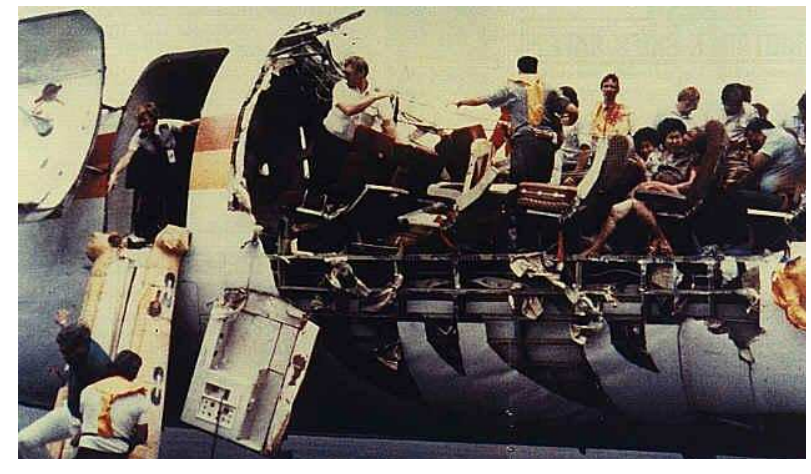

Figura 2.5: Boeing 737 com ruptura na fuselagem

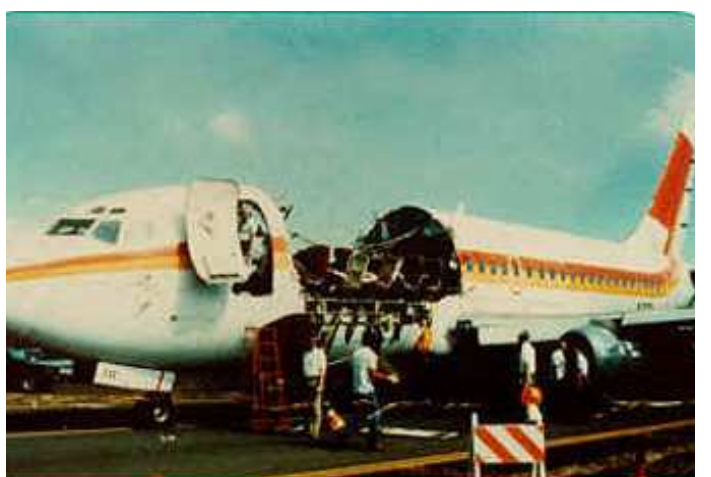

Figura 2.6: Boeing 737

Os acontecimentos dessa natureza ressaltaram a probabilidade de fissuras ocasionarem fratura catastrófica em diversas estruturas. Com o advento da Mecânica da Fratura, originouse então uma nova filosofia de projeto, na qual uma estrutura não é necessariamente um meio contínuo, podendo a mesma conter defeitos (devido ao processo de fabricação), fissuras, vazios, etc. que comprometem a resistência do material. 


\subsection{Mecânica da Fratura: Conceitos Básicos}

Entre os conceitos importantes da Mecânica da Fratura destaca-se a descrição do comportamento das fissuras no que diz respeito ao tipo de deformação de sua superfície. Notou-se então que existiam três movimentos independentes entre as faces de uma determinada fissura, definindo-se estes como sendo "modos de abertura". A figura abaixo retrata estes três modos:

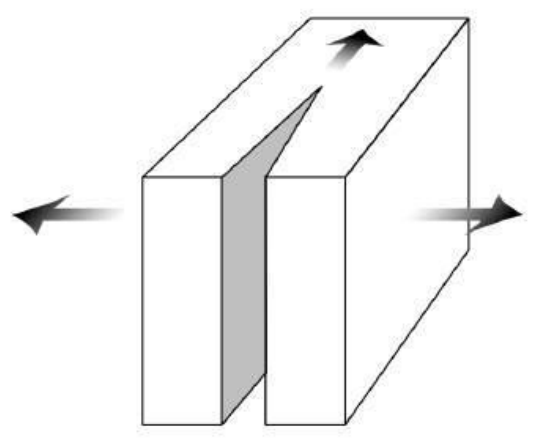

(a)

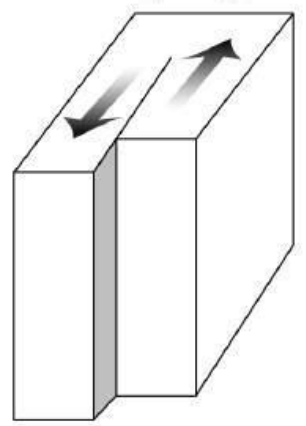

(b)

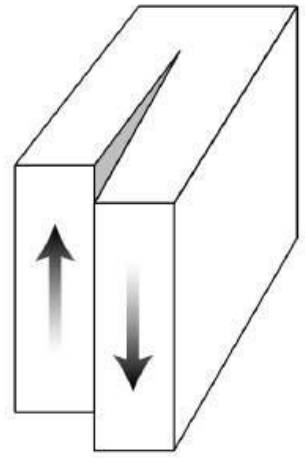

(c)

Figura 2.7 - Modos de abertura de fissuras

a) Modo I ou de abertura: corresponde ao movimento de abertura de fissura na direção perpendicular ao seu plano, por efeito das tensões de tração nessa direção. É o modo mais perigoso no que se refere à propagação de fissura;

b) Modo II ou de cisalhamento plano: corresponde ao escorregamento relativo entre as faces da fissura por efeito de cisalhamento na direção paralela à do defeito;

c) Modo III ou de cisalhamento anti-plano: corresponde ao escorregamento relativo entre as faces da fissura por efeito de cisalhamento na direção transversal à do defeito. 
Gdoutos (1993) observa que as fissuras em corpos solicitados são verdadeiros concentradores de tensões e que usualmente estas tensões amplificadas produzem deformações inelásticas e outros efeitos não-lineares próximos à ponta da fissura (exceto materiais frágeis ideais). Porém, existem situações onde a extensão das deformações inelásticas e efeitos não-lineares são muito pequenos, comparado com o tamanho do corpo (componente estrutural) sendo este o motivo da presente pesquisa abordar somente fissuras em comportamento elástico-linear.

\subsubsection{Função Tensão de Airy e as Funções Complexas de Westergaard na Resolução de Problemas da Mecânica da Fratura Elástico-Linear}

Timoshenko e Goodier (1980) introduzem a função tensão de Airy $\psi$ para a solução de problemas da elasticidade bidimensional e, em particular, para a determinação dos campos de tensão e deslocamento em problemas da Mecânica da Fratura. Da função de Airy decorrem as componentes de tensão expressas da seguinte forma:

$$
\sigma_{x}=\frac{\partial^{2} \psi}{\partial y^{2}} \quad \sigma_{y}=\frac{\partial^{2} \psi}{\partial x^{2}} \quad \tau_{x y}=-\frac{\partial^{2} \psi}{\partial x \cdot \partial y}
$$

A função $\psi$ constitui solução da equação bi-harmônica representante do problema da elasticidade plana (detalhes desta equação se encontram no apêndice A):

$$
\begin{aligned}
& \nabla^{4} \psi=\nabla^{2}\left(\nabla^{2} \psi\right)=\left(\frac{\partial^{2}}{\partial x^{2}}+\frac{\partial^{2}}{\partial y^{2}}\right) \cdot\left(\frac{\partial^{2}}{\partial x^{2}}+\frac{\partial^{2}}{\partial y^{2}}\right) \cdot \psi= \\
& =\left(\frac{\partial^{4}}{\partial x^{4}}+2 \frac{\partial^{4}}{\partial x^{2} y^{2}}+\frac{\partial^{4}}{\partial y^{4}}\right) \cdot \psi=\frac{\partial^{4} \psi}{\partial x^{4}}+2 \frac{\partial^{4} \psi}{\partial x^{2} y^{2}}+\frac{\partial^{4} \psi}{\partial y^{4}}=0 \\
& \therefore \quad \nabla^{2}=\left(\frac{\partial^{2}}{\partial x^{2}}+\frac{\partial^{2}}{\partial y^{2}}\right) \quad \text { (Operador Harmônico de Laplace) }
\end{aligned}
$$


Uma vez que toda função harmônica é bi-harmônica, a solução $\psi$ de uma dada equação bi-harmônica pode ser dada como uma combinação de funções harmônicas $\Omega_{i} \quad(i=0,1,2):$

$\psi=\Omega_{0}+\Omega_{1} \cdot y+\Omega_{2} \cdot x$

$\nabla^{2} \Omega_{i}=\left(\frac{\partial^{2}}{\partial x^{2}}+\frac{\partial^{2}}{\partial y^{2}}\right) \cdot \Omega_{i}=\frac{\partial^{2} \Omega_{i}}{\partial x^{2}}+\frac{\partial^{2} \Omega_{i}}{\partial y^{2}}=0$

O problema da singularidade do campo de tensão introduzida pela presença de fissura está ilustrado na figura 2.8, consistindo em uma chapa de dimensões infinitas sob tensões de tração biaxiais aplicadas no infinito:

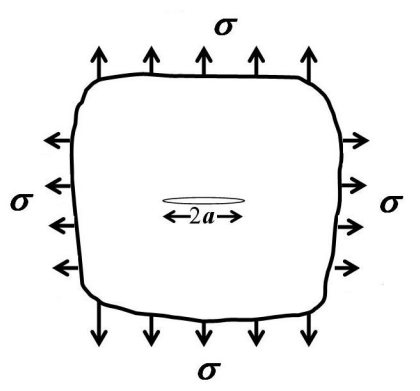

Figura 2.8 - Chapa de tamanho infinito submetida a carregamento biaxial

Neste caso o sistema de referência $(\boldsymbol{x}, \boldsymbol{y})$ para um dado ponto $\mathbf{P}$ é assumido, tendo como origem o semi-comprimento da fissura, como mostra a figura 2.9. Desta maneira, a distância deste ponto $\mathbf{P}$ até a origem do sistema é representada pela variável $\mathbf{z}$ e a distância entre a ponta da fissura até este mesmo ponto $\mathbf{P}$ é representada pela variável $\boldsymbol{\xi}$ :

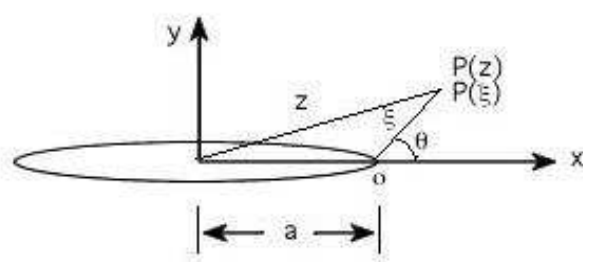

Figura 2.9 - Sistema de coordenadas do campo de tensões e deslocamentos 
Para a resolução deste problema, Westergaard (1939) apud Alliabadi e Rooke (1991) introduziu a seguinte função representativa da distribuição de tensão no problema:

$$
Z_{I}=Z_{I}(z)=Z_{I}(x, y)=\frac{\sigma \cdot z}{\sqrt{z^{2}-a^{2}}}=\frac{\sigma}{\frac{1}{z} \cdot \sqrt{z^{2}-a^{2}}}=\frac{\sigma}{\sqrt{\frac{z^{2}}{z^{2}}-\frac{a^{2}}{z^{2}}}} \Rightarrow Z_{I}(z)=\frac{\sigma}{\sqrt{1-\left(\frac{a}{z}\right)^{2}}}
$$

Importante ressaltar que a parte "real" desta equação satisfaz às seguintes condições de contorno:

a1) Tensão nula nas faces da fissura (excluindo as extremidades/pontas da fissura). Considerando primeiramente um ponto na origem do eixo da fissura e posteriormente um ponto próximo à ponta da fissura.

Origem do sistema $(\boldsymbol{x}, \boldsymbol{y})$ :

$Z_{I}(z)=Z_{I}(0)=\frac{\sigma}{\sqrt{1-\left(\frac{a}{0}\right)^{2}}}=\frac{\sigma}{\sqrt{1-\infty}}=\frac{\sigma}{\sqrt{-\infty}}=\frac{\sigma}{\infty \cdot \sqrt{-1}}=\frac{\sigma}{\infty \cdot i}=$

$\Rightarrow$ propriedade de divisão dos números complexos $\Rightarrow \frac{0}{\infty}-\frac{\sigma}{\infty} i=$

$=0-0 i \Rightarrow \operatorname{Re}\left[Z_{I}(0)\right]=0$

Ponto próximo à ponta da fissura, ou seja, um valor próximo de $\boldsymbol{a}$ (semi-comprimento da fissura):

$$
\begin{array}{r}
Z_{I}(z)=Z_{I}(0,9999 a)=\frac{\sigma}{\sqrt{1-\left(\frac{a}{0,9999 a}\right)^{2}}}=\frac{\sigma}{\sqrt{1-1,0002}}=\frac{\sigma}{0,014143 \cdot i}=\frac{(0-\sigma i)}{0,014143}=0-70,7 \cdot i \\
\Rightarrow \quad \operatorname{Re}\left[Z_{I}(0,9999 \ldots \cdot a)\right]=0
\end{array}
$$


b1) Considerando-se um ponto no infinito, o valor de tensão neste ponto é o valor da tensão aplicado na chapa.

$$
Z_{I}(z)=Z_{I}(\infty)=\frac{\sigma}{\sqrt{1-\left(\frac{a}{\infty}\right)^{2}}}=\frac{\sigma}{\sqrt{1-0}}=\sigma=\sigma+0 \cdot i \quad \Rightarrow \quad \operatorname{Re}\left[Z_{I}(\infty)\right]=\sigma
$$

c1) Tensão tendendo ao infinito na ponta da fissura:

$$
Z_{I}(z)=Z_{I}(a)=\frac{\sigma}{\sqrt{1-\left(\frac{a}{a}\right)^{2}}}=\frac{\sigma}{\sqrt{1-1}}=\frac{\sigma}{0}=\infty=\infty+0 \cdot i \Rightarrow \operatorname{Re}\left[Z_{I}(a)\right]=\infty
$$

De maneira análoga, pode-se definir uma função de Westergaard similar à (2.6) para representar o problema da singularidade e condições de contorno para o modo II de abertura.

$$
Z_{I I}=Z_{I I}(z)=Z_{I I}(x, y)=\frac{\tau \cdot i}{\sqrt{1-\left(\frac{a}{z}\right)^{2}}}
$$

Neste caso, a parte “imaginária” da função acima representa os valores de tensão:

a2) Tensão nula na face da fissura (excluindo as extremidades/pontas da fissura). Considerando primeiramente um ponto na origem do eixo da fissura e posteriormente um ponto próximo à ponta da fissura.

$$
Z_{I I}(z)=Z_{I I}(0)=\frac{\tau \cdot i}{\sqrt{1-\left(\frac{a}{0}\right)^{2}}}=\frac{\tau \cdot i}{\sqrt{1-\infty}}=\frac{\tau \cdot i}{\infty \cdot \sqrt{-1}}=\frac{\tau \cdot \dot{\lambda}}{\infty \cdot \dot{\lambda}}=0+0 i \Rightarrow \operatorname{Im}\left[Z_{I I}(0)\right]=0
$$




$$
\begin{gathered}
Z_{I I}(z)=Z_{I I}(0,9999 a)=\frac{\tau \cdot i}{\sqrt{1-\left(\frac{a}{0,9999 a}\right)^{2}}}=\frac{\tau \cdot \dot{\lambda}}{0,014143 \cdot \dot{\lambda}}=70,7 \ldots+0 \cdot i \\
\Rightarrow \quad \operatorname{Im}\left[Z_{I I}(0,9999 a)\right]=0
\end{gathered}
$$

b2) Considerando-se um ponto no infinito, o valor de tensão neste ponto é o valor da tensão aplicada na chapa.

$$
Z_{I I}(z)=Z_{I I}(\infty)=\frac{\tau \cdot i}{\sqrt{1-\left(\frac{a}{\infty}\right)^{2}}}=\frac{\tau \cdot i}{\sqrt{1-0}}=\tau \cdot i=0+\tau \cdot i \quad \Rightarrow \quad \operatorname{Im}\left[Z_{I I}(\infty)\right]=\tau
$$

c2) Tensão tendendo ao infinito na ponta da fissura:

$$
Z_{I I}(z)=Z_{I I}(a)=\frac{\tau \cdot i}{\sqrt{1-\left(\frac{a}{a}\right)^{2}}}=\frac{\tau \cdot i}{\sqrt{1-1}}=\frac{\tau \cdot i}{0}=\infty \cdot i=0+\infty \cdot i \Rightarrow \operatorname{Im}\left[Z_{I I}(a)\right]=\infty
$$

Com base nas equações (2.6) e (2.11), assim como nas verificações expostas para cada uma delas, Gdoutos (1993) resume as condições de contorno em tensões nas faces da fissura de um problema da Mecânica da Fratura mediante a seguinte equação:

$\sigma+\tau \cdot i=0 \quad$ para $\quad y=0 \quad e \quad-a<x<+a$

Tendo como base estas considerações, define-se então a natureza da equação geral representativa da distribuição do campo de tensões de um problema com presença de singularidade:

$Z=Z(z)=Z(x, y)=\operatorname{Re} Z(x, y)+i \cdot \operatorname{Im} Z(x, y)$ 
Uma vez definida a natureza da distribuição do campo de tensões, Westergaard (1939) propôs as seguintes funções de tensão de Airy para a resolução de problemas da elasticidade bidimensional regidas pela equação bi-harmônica (2.2) e consistindo em meio infinito contendo fissura submetida aos modos I e II de abertura:

$\psi_{I}=\operatorname{Re} \tilde{\tilde{Z}}_{I}+y \cdot \operatorname{Im} \tilde{Z}_{I} \quad($ problema simétrico - modo I de abertura)

$\psi_{I I}=y \cdot \operatorname{Im} \tilde{Z}_{I I} \quad$ ( problema anti-simétrico - modo II de abertura)

Nota-se que para a dedução destas duas equações faz-se necessário conhecer as relações (2.20) a (2.23):

$$
\begin{aligned}
& Z^{\prime}=\frac{d Z}{d z} \\
& \tilde{Z}=\int Z d z \\
& Z^{\prime}=\frac{d Z}{d z} \text { (integrando ambos os lados) } \Rightarrow Z=\int\left(\frac{d}{d z} Z\right) d z=\frac{d}{d z} \int Z d z \Rightarrow Z=\frac{d}{d z} \tilde{Z} \\
& \tilde{Z}=\int Z d z \text { utilizando }(2.22) \Rightarrow \tilde{Z}=\int\left(\frac{d}{d z} \tilde{Z}\right) d z=\frac{d}{d z} \int \tilde{Z} d z=\frac{d}{d z} \tilde{Z} \Rightarrow \tilde{Z}=\frac{d}{d z} \tilde{Z}
\end{aligned}
$$

Acrescentam-se às relações anteriores as condições de Cauchy-Riemann (ver Apêndice B) para funções analíticas de uma variável complexa. Essas condições são dadas abaixo:

$$
\begin{aligned}
& \operatorname{Re} Z^{\prime}=\frac{d \operatorname{Re} Z}{d x}=\frac{d \operatorname{Im} Z}{d y} \\
& \operatorname{Im} Z^{\prime}=\frac{d \operatorname{Im} Z}{d x}=-\frac{d \operatorname{Re} Z}{d y}
\end{aligned}
$$

A dedução das funções tensões de Westergaard (2.18) e (2.19) é apresentada no Apêndice C. 


\subsubsection{Equações das Tensões em Função de uma Variável Complexa - Modos I e II de}

\section{Abertura}

Derivando a função complexa (2.18) introduzida por Westergaard e utilizando as condições de Cauchy-Riemann (2.24) e (2.25), obtêm-se as tensões para o modo I de abertura:

$$
\begin{aligned}
& \sigma_{x}=\frac{d^{2} \psi_{I}}{d y^{2}}=\frac{d}{d y}\left(\frac{d \operatorname{Re} \tilde{Z}_{I}}{d y}+\frac{d y \cdot \operatorname{Im} \tilde{Z}_{I}}{d y}\right)=\frac{d}{d y}\left[-\operatorname{Im} \tilde{Z}_{I}+\left(1 \cdot \operatorname{Im} \tilde{Z}_{I}+y \cdot \operatorname{Re} Z_{I}\right)\right]=\frac{d}{d y}\left(y \cdot \operatorname{Re} Z_{I}\right) \\
& \sigma_{x}=1 \cdot \operatorname{Im} Z_{I}+y \cdot\left(-\operatorname{Re} Z_{I}^{\prime}\right)=\operatorname{Im} Z_{I}-y \cdot \operatorname{Re} Z_{I}^{\prime} \Rightarrow \sigma_{x}=\operatorname{Im} Z_{I}-y \cdot \operatorname{Re} Z^{\prime}
\end{aligned}
$$

$\sigma_{y}=\frac{d^{2} \psi_{I}}{d x^{2}}=\frac{d}{d x}\left(\frac{d \operatorname{Re} \tilde{Z}_{I}}{d x}+\frac{d y \cdot \operatorname{Im} \tilde{Z}_{I}}{d x}\right)=\frac{d}{d x}\left(\operatorname{Re} \tilde{Z}_{I}+y \cdot \operatorname{Im} Z_{I}\right)=\operatorname{Re} Z_{I}+y \cdot \operatorname{Im} Z_{I}^{\prime}$

$\sigma_{y}=\operatorname{Re} Z_{I}+y \cdot \operatorname{Im} Z_{I}^{\prime}$

$$
\begin{aligned}
& \tau_{x y}=-\frac{d^{2} \psi_{I}}{d x d y}=-\frac{d^{2}}{d x d y}\left(\operatorname{Re} \tilde{\tilde{Z}}_{I}+y \cdot \operatorname{Im} \tilde{Z}_{I}\right)=-\frac{d}{d y}\left(\frac{d \operatorname{Re} \tilde{\tilde{Z}}_{I}}{d x}+\frac{d y \cdot \operatorname{Im} \tilde{Z}_{I}}{d x}\right)=-\frac{d}{d y}\left(\operatorname{Re} \tilde{Z}_{I}+y \cdot \operatorname{Im} Z_{I}\right) \\
& \tau_{x y}=-\frac{d \operatorname{Re} \tilde{Z}_{I}}{d y}-\frac{d y \cdot \operatorname{Im} Z_{I}}{d y}=\operatorname{Im} Z_{I}-\left(1 \cdot \operatorname{Im} Z_{I}+y \cdot \operatorname{Re} Z_{I}^{\prime}\right) \Rightarrow \tau_{x y}=-y \cdot \operatorname{Re} Z_{I}^{\prime}
\end{aligned}
$$

De maneira análoga, utilizando a função $\psi_{I I}$ (2.19) obtêm-se as funções de tensão para o modo II de abertura:

$$
\begin{aligned}
\sigma_{x} & =\frac{d^{2} \psi_{I I}}{d y^{2}}=\frac{d^{2}\left(y \cdot \operatorname{Im} \tilde{Z}_{I I}\right)}{d y^{2}}=\frac{d\left(y \cdot \operatorname{Re} Z_{I I}+\operatorname{Im} \tilde{Z}_{I I}\right)}{d y} \Rightarrow \\
& \Rightarrow \quad\left(\operatorname{Re} Z_{I I}-y \operatorname{Im} Z^{\prime}\right)+\operatorname{Re} Z_{I I}=2 \cdot \operatorname{Re} Z_{I I}-y \cdot \operatorname{Im} Z_{I I}^{\prime} \Rightarrow \sigma_{x}=2 \cdot \operatorname{Re} Z_{I I}-y \cdot \operatorname{Im} Z_{I I}^{\prime}
\end{aligned}
$$




$$
\begin{aligned}
& \sigma_{y}=\frac{d^{2} \psi_{I I}}{d x^{2}}=\frac{d}{d x}\left(\frac{y \cdot d \operatorname{Im} \tilde{Z}_{I I}}{d x}\right)=y \frac{d \operatorname{Im} Z_{I I}}{d x} \Rightarrow \sigma_{y}=y \cdot \operatorname{Im} Z_{I I}^{\prime} \\
& \tau_{x y}=-\frac{d^{2} \psi_{I I}}{d x d y}=-\frac{d}{d x}\left[\frac{d\left(y \operatorname{Im} \tilde{Z}_{I I}\right)}{d y}\right]=-\frac{d\left(y \operatorname{Re} Z_{I I}+\operatorname{Im} \tilde{Z}_{I I}\right)}{d x} \Rightarrow \tau_{x y}=-y \operatorname{Re} Z_{I I}^{\prime}-\operatorname{Im} Z_{I I}
\end{aligned}
$$

\subsubsection{Componentes de Deslocamento em Função de uma Variável Complexa - Modos I e II de Abertura - EPT e EPD}

Obtidas as tensões, faz-se necessário inseri-las nas equações que descrevem as deformações no problema bidimensional para que seja possível a obtenção das relações entre tensões e deslocamentos. As relações de interesse entre deformações, deslocamentos e tensões considerando-se material isotrópico, homogêneo e linear-elástico são as seguintes:

$$
\begin{aligned}
& \varepsilon_{x}=\frac{d u}{d x} \\
& \varepsilon_{y}=\frac{d v}{d y} \\
& \gamma_{x y}=\frac{d u}{d y}+\frac{d v}{d x} \\
& \varepsilon_{x}=\frac{1}{E}\left[\sigma_{x}-v\left(\sigma_{y}+\sigma_{z}\right)\right] \\
& \varepsilon_{y}=\frac{1}{E}\left[\sigma_{y}-v\left(\sigma_{x}+\sigma_{z}\right)\right] \\
& \gamma_{x y}=\frac{\tau_{x y}}{G}
\end{aligned}
$$

com

$$
G=\frac{E}{2(1+v)}
$$


Substituindo (2.32) em (2.35), resulta:

$$
\begin{aligned}
& \frac{d u}{d x}=\frac{1}{E}\left[\sigma_{x}-v\left(\sigma_{y}+\sigma_{z}\right)\right] \Rightarrow d u \\
& \Rightarrow \int \frac{1}{E}\left[\sigma_{x}-v\left(\sigma_{y}+\sigma_{z}\right)\right] d x \Rightarrow \int d u=\int \frac{1}{E}\left[\sigma_{x}-v\left(\sigma_{y}+\sigma_{z}\right)\right] d x \\
& \Rightarrow u=\frac{1}{E}\left[\int \sigma_{x} d x-v \int\left(\sigma_{y}+\sigma_{z}\right) d x\right] d x \Rightarrow
\end{aligned}
$$

De maneira análoga, substituindo (2.33) em (2.36) obtêm-se:

$$
\frac{d v}{d y}=\frac{1}{E}\left[\sigma_{y}-v\left(\sigma_{x}+\sigma_{z}\right)\right] \Rightarrow v=\int \frac{1}{E}\left[\sigma_{y}-v\left(\sigma_{x}+\sigma_{z}\right)\right] d y \Rightarrow v=\frac{1}{E}\left[\int \sigma_{y} \partial y-v \int\left(\sigma_{x}+\sigma_{z}\right) d y\right]
$$

Obtidas as expressões que relacionam componentes de deslocamentos e tensões, podese então definir as expressões de deslocamento para estado plano de tensão (EPT) $\left(\sigma_{z}=0\right) \mathrm{e}$ para estado plano de deformação (EPD) $\left(\sigma_{\mathrm{z}}=v \cdot\left(\sigma_{\mathrm{x}}+\sigma_{\mathrm{y}}\right)\right)$, tanto para modo I quanto para o modo II de abertura. Estas expressões têm sua dedução detalhada no apêndice D e são resumidas na tabela 2.1 .

Tabela 2.1 - Expressões dos Deslocamentos em Função de Z para Modos I e II de abertura - EPT e EPD

\begin{tabular}{|c|c|c|}
\hline $\begin{array}{c}\text { Modo de } \\
\text { Abertura }\end{array}$ & EPT & EPD \\
\hline \multirow{2}{*}{ I } & $2 G u=\frac{(1-v)}{(v+1)} \cdot \operatorname{Re} \tilde{Z}_{I}-y \cdot \operatorname{Im} Z_{I}$ & $2 G u=(1-2 v) \cdot \operatorname{Re} \tilde{Z}_{I}-y \cdot \operatorname{Im} Z_{I}$ \\
\cline { 2 - 4 } & $2 G v=\frac{2}{(1+v)} \cdot \operatorname{Im} \tilde{Z}_{I}-\operatorname{Re} Z_{I} \cdot y$ & $2 G v=2 \cdot(1-v) \cdot \operatorname{Im} \tilde{Z}_{I}-\operatorname{Re} Z_{I} \cdot y$ \\
\hline \multirow{2}{*}{ II } & $2 G u=\frac{2}{(v+1)} \cdot \operatorname{Re} \tilde{Z}_{I I}+y \cdot \operatorname{Im} Z_{I I}$ & $2 G u=2(1-v) \cdot \operatorname{Re} \tilde{Z}_{I I}-y \cdot \operatorname{Im} Z_{I I}$ \\
\cline { 2 - 4 } & $2 G v=\frac{(1-v)}{(1+v)} \cdot \operatorname{Im} \tilde{Z}_{I I}-y \cdot \operatorname{Re} Z_{I I}$ & $2 G v=(1-2 v) \cdot \operatorname{Im} \tilde{Z}_{I I}-y \cdot \operatorname{Re} Z_{I I}$ \\
\hline
\end{tabular}




\subsubsection{Relações entre os Campos de Tensão e Deslocamento na Vizinhança da Ponta da Fissura - Modos I e II de Abertura}

Como a tensão de interesse é aquela próxima à ponta da fissura, inicialmente torna-se interessante transladar a origem do sistema de coordenadas (ver figura 2.9) para a ponta da fissura, mediante a relação:

$$
z=\xi+a
$$

Em seguida, inserindo (2.41) em (2.6) tem-se que:

$$
Z_{I}=\frac{\sigma \cdot z}{\sqrt{z^{2}-a^{2}}}=\frac{\sigma \cdot(\xi+a)}{\sqrt{(\xi+a)^{2}-a^{2}}}=\frac{\sigma \cdot(\xi+a)}{\sqrt{\xi^{2}+2 \xi a}}
$$

Como a função $\mathrm{Z}_{\mathrm{I}}$ determina o campo de tensão muito próximo à ponta da fissura, considera-se então a distância $(\xi)$ da ponta da fissura até um dado ponto $\mathrm{P}$ em questão como sendo muito inferior ao semi-comprimento (a) da fissura:

$\xi<<a$

Desta forma:

$$
\begin{aligned}
Z_{I} & =\frac{\sigma \cdot(\not{\xi}+a)}{\sqrt{\xi^{\prime}+2 \xi a}}=\frac{\sigma \cdot a}{\sqrt{2 \xi a}} \cdot\left(\frac{\sqrt{\pi a}}{\sqrt{\pi a}}\right)=\frac{\sigma \sqrt{\pi a}}{\sqrt{2 \pi \xi}} \quad \Leftrightarrow \quad \therefore \quad \sigma \sqrt{\pi a}=K_{I} \\
& \Rightarrow \quad Z_{I}=\frac{K_{I}}{\sqrt{2 \pi \xi}}=\frac{K_{I}}{\sqrt{2 \pi}} \cdot \xi^{-1 / 2}
\end{aligned}
$$

Remete-se ao desenvolvimento complementar no Apêndice E, obtendo as equações que definem os campos de tensão e deslocamento, em função das coordenadas $\boldsymbol{r}$ e $\boldsymbol{\theta}$, em uma região próxima à ponta da fissura (modos I e II de abertura). A tabela 2.2 reúne as relações de interesse. Observa-se que os parâmetros $\mathrm{K}_{\mathrm{I}}$ e $\mathrm{K}_{\mathrm{II}}$ constituem fatores de intensidade de tensão e serão tratados logo em seguida. 
Tabela 2.2 - Expressões Deslocamento x Tensões em Função de $\boldsymbol{r}$ e $\boldsymbol{\theta}$, para Modos I e II de abertura - EPT e EPD

\begin{tabular}{|c|c|c|}
\hline $\begin{array}{l}\text { Modo de } \\
\text { Abertura }\end{array}$ & Tensões & Deslocamentos \\
\hline I & $\begin{array}{l}\sigma_{x}=\frac{K_{I}}{\sqrt{2 \pi r}} \cdot \cos \left(\frac{\theta}{2}\right) \cdot\left[1-\operatorname{sen}\left(\frac{\theta}{2}\right) \cdot \operatorname{sen}\left(\frac{3 \theta}{2}\right)\right] \\
\sigma_{y}=\frac{K_{I}}{\sqrt{2 \pi r}} \cdot \cos \left(\frac{\theta}{2}\right) \cdot\left[1+\operatorname{sen}\left(\frac{\theta}{2}\right) \cdot \operatorname{sen}\left(\frac{3 \theta}{2}\right)\right] \\
\tau_{x y}=\frac{K_{I}}{\sqrt{2 \pi r}} \cdot \cos \left(\frac{\theta}{2}\right) \cdot\left[\operatorname{sen}\left(\frac{\theta}{2}\right) \cdot \cos \left(\frac{3 \theta}{2}\right)\right]\end{array}$ & $\begin{array}{c}u=\frac{K_{I}}{2 G} \cdot \sqrt{\frac{r}{2 \pi}} \cdot \cos \left(\frac{\theta}{2}\right) \cdot(\kappa-\cos \theta) \\
v=\frac{K_{I}}{2 G} \cdot \sqrt{\frac{r}{2 \pi}} \cdot \operatorname{sen}\left(\frac{\theta}{2}\right) \cdot(\kappa-\cos \theta)\end{array}$ \\
\hline II & $\begin{array}{l}\sigma_{x}=\frac{-K_{I I}}{\sqrt{2 \pi r}} \cdot \operatorname{sen}\left(\frac{\theta}{2}\right) \cdot\left[2+\cos \left(\frac{\theta}{2}\right) \cdot \cos \left(\frac{3 \theta}{2}\right)\right] \\
\sigma_{y}=\frac{K_{I I}}{\sqrt{2 \pi r}} \cdot \operatorname{sen}\left(\frac{\theta}{2}\right) \cdot \cos \left(\frac{\theta}{2}\right) \cdot \cos \left(\frac{3 \theta}{2}\right) \\
\tau_{x y}=\frac{K_{I I}}{\sqrt{2 \pi r}} \cdot \cos \left(\frac{\theta}{2}\right) \cdot\left[1-\operatorname{sen}\left(\frac{\theta}{2}\right) \cdot \operatorname{sen}\left(\frac{3 \theta}{2}\right)\right]\end{array}$ & $\begin{array}{l}u=\frac{K_{I I}}{2 G} \cdot \sqrt{\frac{r}{2 \pi}} \cdot \operatorname{sen}\left(\frac{\theta}{2}\right) \cdot(2+\kappa+\cos \theta) \\
v=\frac{K_{I I}}{2 G} \cdot \sqrt{\frac{r}{2 \pi}} \cdot \cos \left(\frac{\theta}{2}\right) \cdot(2-\kappa-\cos \theta)\end{array}$ \\
\hline
\end{tabular}

\subsubsection{Equações que Definem os Campos de Tensão e Deslocamento próximos à Ponta da}

\section{Fissura - Modo III de Abertura}

De menor interesse para análises planas, mas no sentido de complementar a abordagem, apresenta-se as equações que definem os campos de tensão e deslocamento para o modo III de abertura. As deduções destas equações se encontram no Apêndice F.

$$
\tau_{x z}=\operatorname{Im} Z_{I I I}^{\prime}=-\frac{K_{I I I}}{\sqrt{2 \pi r}} \cdot \operatorname{sen}\left(\frac{\theta}{2}\right)
$$




$$
\begin{gathered}
\tau_{y z}=\operatorname{Re} Z_{I I I}^{\prime}=\frac{K_{I I I}}{\sqrt{2 \pi r}} \cdot \cos \left(\frac{\theta}{2}\right) \\
w=\frac{\operatorname{Im} Z_{I I I}}{G}=\frac{K_{I I I}}{G} \cdot \sqrt{\frac{2 r}{\pi}} \cdot \operatorname{sen}\left(\frac{\theta}{2}\right)
\end{gathered}
$$

Nas relações anteriores aparece o fator de intensidade de tensão $\mathrm{K}_{\mathrm{III}}$.

\subsubsection{O Fator de Intensidade de Tensão}

As equações que descrevem os campos de tensão para os três modos de abertura se caracterizam pela multiplicação de dois fatores que envolvem interpretações distintas. Conforme a equação (2.48), o primeiro fator refere-se a uma constante que descreve os campos de tensão, deformação e deslocamentos na região imediatamente à frente da ponta de fissura, enquanto que o segundo, dependente de $\boldsymbol{r}$ e $\theta$, é responsável por descrever a distribuição deste campo de tensão em uma região próxima à ponta da fissura, Ferreira (2005).

$$
\sigma_{i j}(r, \theta)=\underbrace{K}_{1^{\circ} \text { parte }} \cdot \underbrace{\frac{f(\theta)}{\sqrt{2 \pi r}}}_{2^{\circ} \text { parte }} \quad \text { onde } \quad \therefore K=\sigma \sqrt{\pi a}
$$

Observando na equação (2.48) que quando $\boldsymbol{r}$ tende a zero, a tensão tende ao infinito e quando $\theta$ tende à zero, $f(\theta)$ tende a um valor unitário, pode-se então definir o fator de intensidade de tensão como:

$$
K=\lim _{r \rightarrow 0} \sqrt{2 \pi r} \cdot \sigma_{i j}(r, \theta)
$$

Pode-se mostrar que o processo limite acima indicado tem por resultado:

$$
K=\sigma \sqrt{\pi a}
$$


onde:

$\sigma$ : é a correspondente tensão de referência aplicada no corpo contendo a fissura e

$a$ : é o semi-comprimento da fissura (ver figura 2.9). 


\section{Método da Partição na Análise de Problemas com Múltiplas Fissuras}

\subsection{Breve Histórico Sobre as Análises de Múltiplas Fissuras}

Uma das alternativas para encontrar-se um método eficaz para resolver problemas com múltiplas fissuras em elasticidade bidimensional foi a utilização das funções desenvolvidas por Green em 1928. Uma importante característica destas funções é que quando bem definidas elas podem conter informações particulares de um sistema como um todo, de modo que a solução final de um problema pode ser obtida pela soma de respostas de uma combinação de subproblemas agindo separadamente. Porém, este tipo de análise se restringe somente a casos de fissuras submetidas ao modo I de abertura, sendo colineares com distâncias periódicas entre elas além de idênticos comprimentos. Diversos tipos de funções 
Green podem ser encontrados em Shivakumar e Forman (1980), Rooke e Tweed (1980), Rooke e Hutchins (1984), Kaya e Erdogan (1980), Hsu e Rudd (1977) e Jones (1974).

Outros métodos para as análises de múltiplas fissuras em problemas similares ao descrito anteriormente foram desenvolvidos. Dentre estes se encontram as funções ponderadoras propostas por Bueckner (1970) e mais amplamente desenvolvidas por Rice (1972) que buscou aplicá-las em abordagem tridimensional. Estas funções proporcionam expressão integral para a determinação do fator de intensidade de tensão. Uma das vantagens apresentadas pelo método refere-se à sua generalização para a determinação de fatores de intensidade de tensão em modo misto, realizada por diversos pesquisadores, dentre eles destacamos Tada, Paris e Irwin (2000) e Bortman e Banks-Sills (1983). Porém a aplicação ainda se limitava à análise de fissuras colineares.

Visando o desenvolvimento de uma metodologia com capacidade de determinação de fatores de intensidade de tensão para problemas contendo múltiplas fissuras, Lachenbruch (1961) utilizou pela primeira vez o Schwartz-Neumann Alternating Method no campo da Mecânica da Fratura para a resolução de problemas com fissuras de canto considerando-se casos de deformação plana. Tal método trata da aplicação do Princípio de Sobreposição, onde a solução final do problema é dada pela soma das soluções de dois subproblemas. Um dos subproblemas refere-se a um meio considerado infinito contendo uma fissura tendo por objetivo capturar a singularidade local do problema. Emprega-se, neste caso, a solução analítica disponível. O segundo subproblema refere-se a um domínio finito e sem fissuras, responsável pela determinação da parcela regular da solução global.

Mas somente com o trabalho de Nishioka e Atluri (1983) que o Método dos Elementos Finitos foi utilizado juntamente com soluções analíticas do Alternating Method para a análise de fissuras, surgindo então o Finite Element Alternating Method, capaz de analisar fissuras submetidas ao modo misto de carregamento com localização qualquer. Porém, um ponto importante a ressaltar refere-se à dificuldade de determinação dos fatores de intensidade de tensão para um número elevado de fissuras uma vez que cada fator de intensidade de tensão está relacionado com os demais. A busca da solução exige, neste caso, um processo iterativo. Deste modo, buscando uma alternativa mais eficiente para a determinação dos fatores de intensidade de tensão em múltiplas fissuras, Andersson, Babuška e Stehlin (1998) propõem um novo método de decomposição denominado Método da Partição (Splitting Method). Tal alternativa consiste na separação do problema original (contendo um conjunto de fissuras) em três subproblemas. O primeiro subproblema consiste no domínio global com as mesmas condições de contorno e forças externas aplicadas do problema original, porém sem fissuras. 
O segundo subproblema, local, considera uma fissura padrão em um meio infinito. O terceiro subproblema é também definido no domínio global, sem fissuras e com as mesmas condições de contorno em deslocamento do problema original, porém com tensões e deslocamentos oriundos do subproblema local impostos em regiões definidas nas vizinhanças das fissuras. A solução advém da soma dos três subproblemas, com a condição adicional de nulidade das tensões ao longo das linhas de fissuras. Tal método oferece entre outras vantagens a possibilidade de paralelização do processamento do problema local e do problema global referente à interação entre fissuras além da convergência exponencial das respostas.

Sendo assim, com o objetivo de detalhar a formulação numérica do Método da Partição, primeiramente são abordados princípios básicos da Mecânica da Fratura que norteiam o Método da Partição.

\subsection{Princípio de Bueckner}

Bueckner (1958) demonstrou que o fator de intensidade de tensão de fissura inserida em sólido submetido a uma força externa qualquer pode também ser determinado mediante a aplicação de uma distribuição de tensões diretamente nas faces da fissura. Tal princípio é válido para material elástico-linear. A sobreposição que justifica essa equivalência é mais especificamente apresentada nos itens abaixo:

a) Considera-se o corpo sob carregamento aplicado, porém sem considerar a presença da fissura, e determina-se a distribuição de tensões na linha da fissura;

b) Considerando-se o corpo agora com a presença da fissura, sendo a mesma submetida a uma tensão de fechamento de intensidade igual àquela determinada no item "a". Obtêm-se então o fator de intensidade de tensão em questão. Como a situação do item "a" não apresenta concentração de tensão, este valor de fator de intensidade de tensão coincide com o do problema original em módulo. Sendo assim, tem-se que $K_{B}=-K_{C}$ conforme mostra a figura 3.1: 


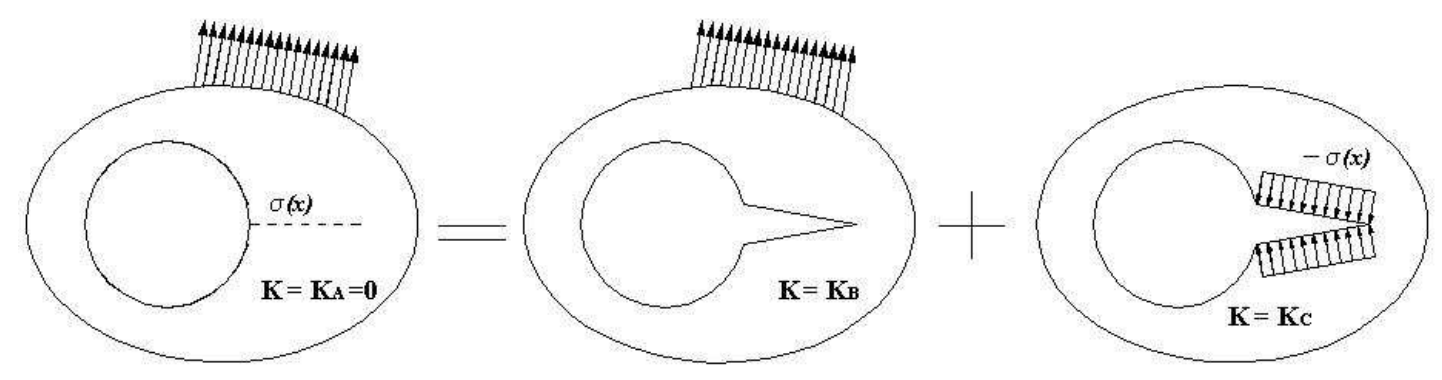

Figura 3.1 - Princípio de Bueckner

\subsection{Princípio de Sobreposição}

Aliabadi e Rooke (1991) destacam o Princípio de Sobreposição como uma das técnicas mais simples e mais comuns na resolução de problemas da Mecânica da Fratura.
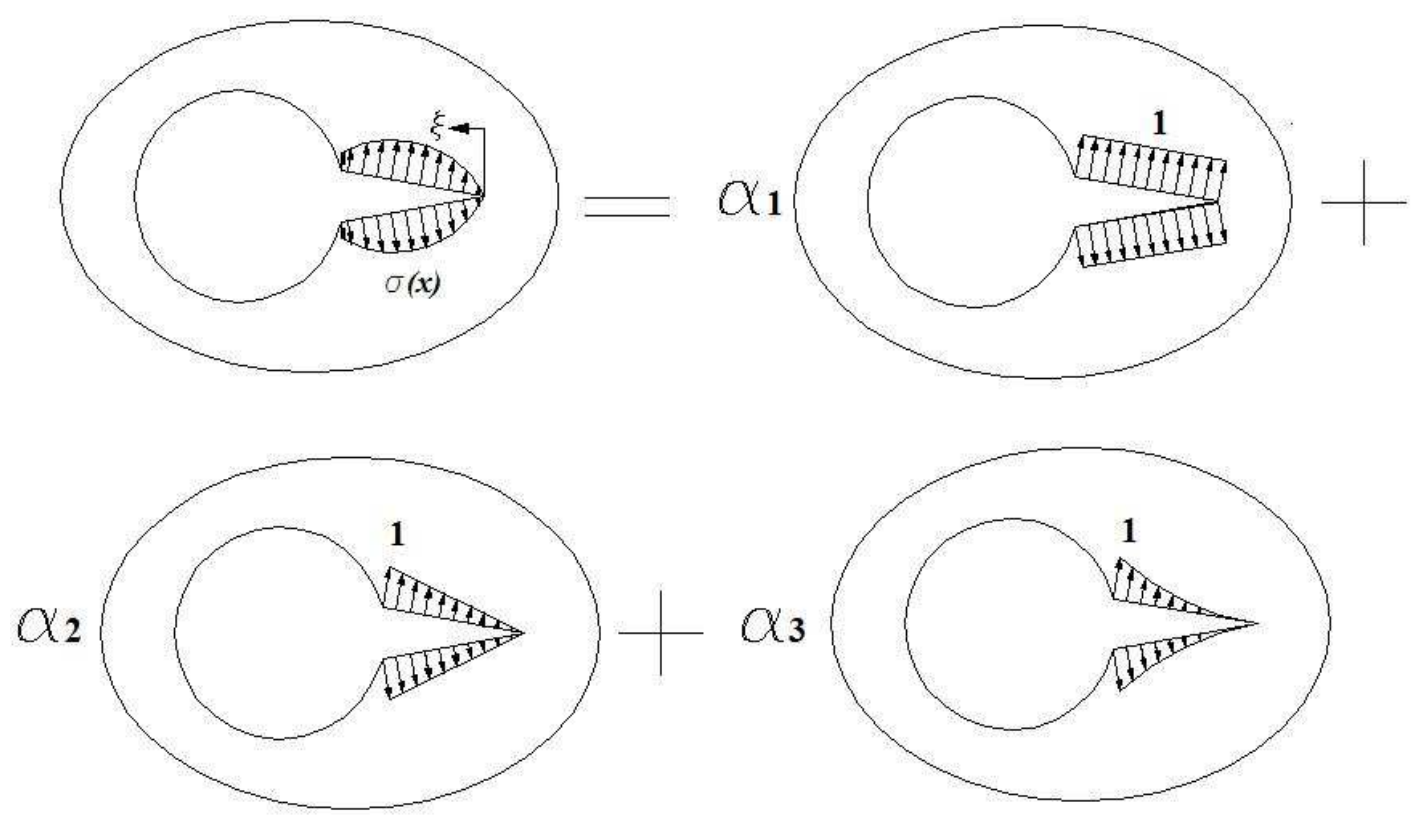

Figura 3.2 - Carregamento na face de uma fissura resolvido

Com o Princípio de Sobreposição

Uma forma de aplicação deste princípio para fins de determinação do fator de intensidade de tensão em situação mais complexa de solicitação é apresentada por Chell (1976) e Grandt Júnior (1978). Para a determinação do fator de intensidade de tensão quando 
nas faces da fissura aplica-se um carregamento distribuído qualquer $\sigma(x)$, considera-se que o mesmo possa ser descrito por uma combinação linear de polinômios da seguinte forma:

$$
Q=\sum_{j=0}^{J} \alpha_{j} \cdot\left(\frac{\xi}{a}\right)^{j}
$$

onde $\alpha_{j}$ são algumas constantes conhecidas, $Q$ o carregamento aproximativo e $\begin{array}{lll}\boldsymbol{a} & \mathrm{o}\end{array}$ comprimento da fissura. Desta forma, o fator de intensidade de tensão é determinado pela soma dos fatores de intensidade de tensão obtidos em cada um dos subproblemas, conforme mostra a figura 3.2 .

O Princípio de Sobreposição possibilita a paralelização do processamento dos fatores de intensidade de tensão de cada subproblema. Outra aplicação importante do princípio, explorada neste trabalho, refere-se aos problemas que envolvem a presença de múltiplas fissuras. Neste caso, os subproblemas de fissuras de mesmas características geométricas são calculados uma única vez, o que auxilia na redução de tempo de processamento total do problema.

\subsection{Princípio de Sobreposição e de Bueckner como Ferramentas do Método da Partição}

Os princípios descritos anteriormente formam a base do Método da Partição para a solução de problemas da Mecânica da Fratura. Para exemplificar, retoma-se o caso de um corpo ausente de fissuras, submetido a um carregamento distribuído nas duas extremidades opostas. Este problema pode ser decomposto em dois subproblemas, conforme mostra a figura 3.3: 


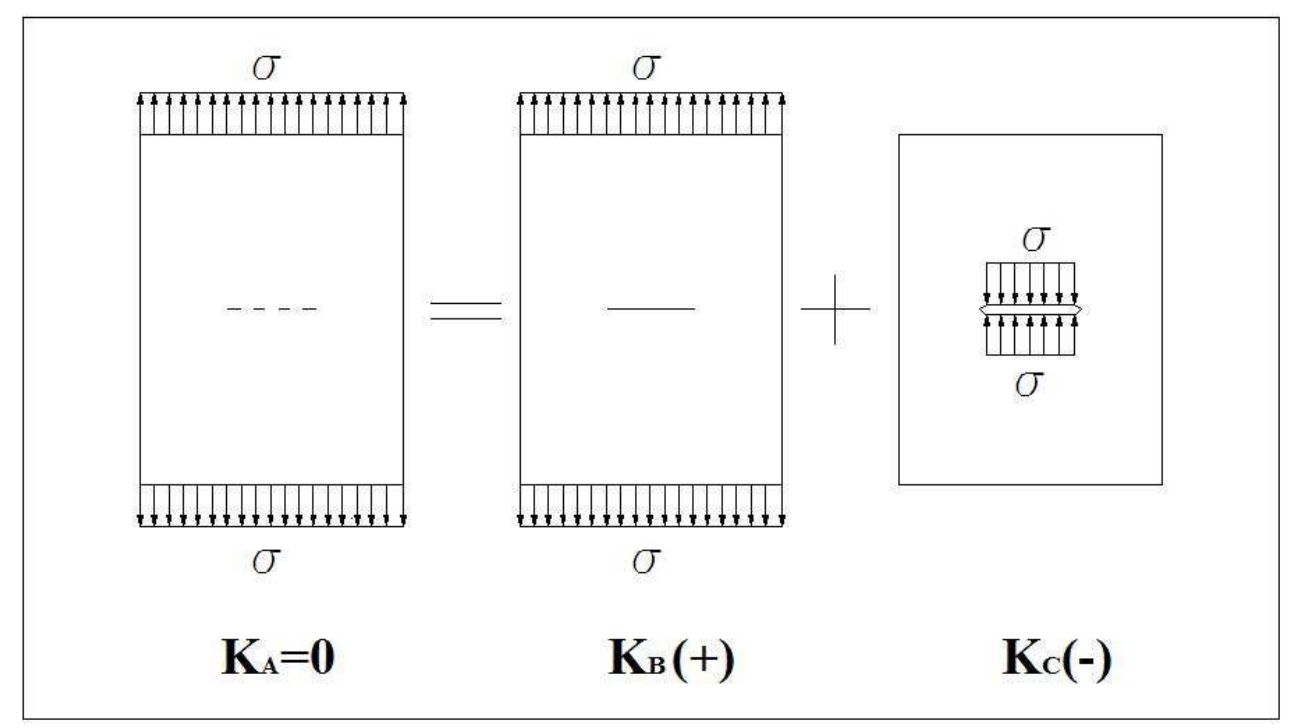

Figura 3.3 - Princípio de Sobreposição e Princípio de Bueckner

Isolando o subproblema $\left(K_{B}\right)$ tem-se que o mesmo representa o caso clássico da Mecânica da Fratura onde o fator de intensidade de tensão fica determinado pela equação (3.2):

$K=C \sigma \sqrt{\pi a}$

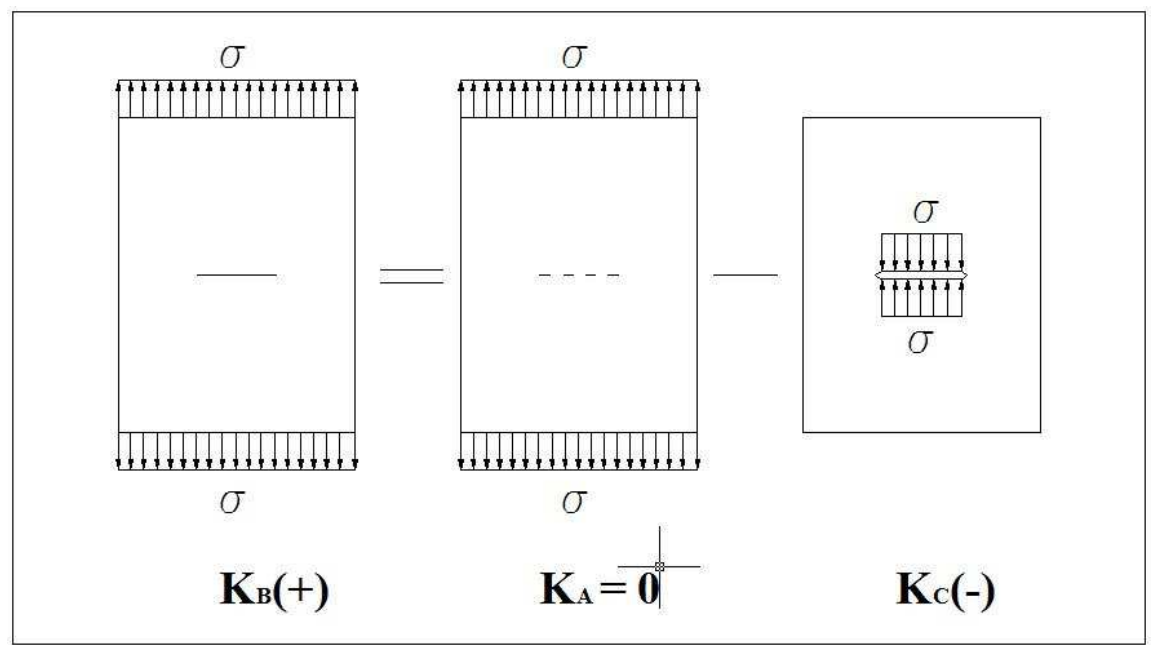

Figura 3.4 - Caso clássico da Mecânica da Fratura dividido em dois subproblemas 
Observando-se a figura 3.4 nota-se que o problema clássico da Mecânica da Fratura está subdividido em dois subproblemas. A sobreposição com sinais positivos é então recuperada aplicando-se a tensão de abertura nas faces de fissura, conforme nesta figura 3.5:

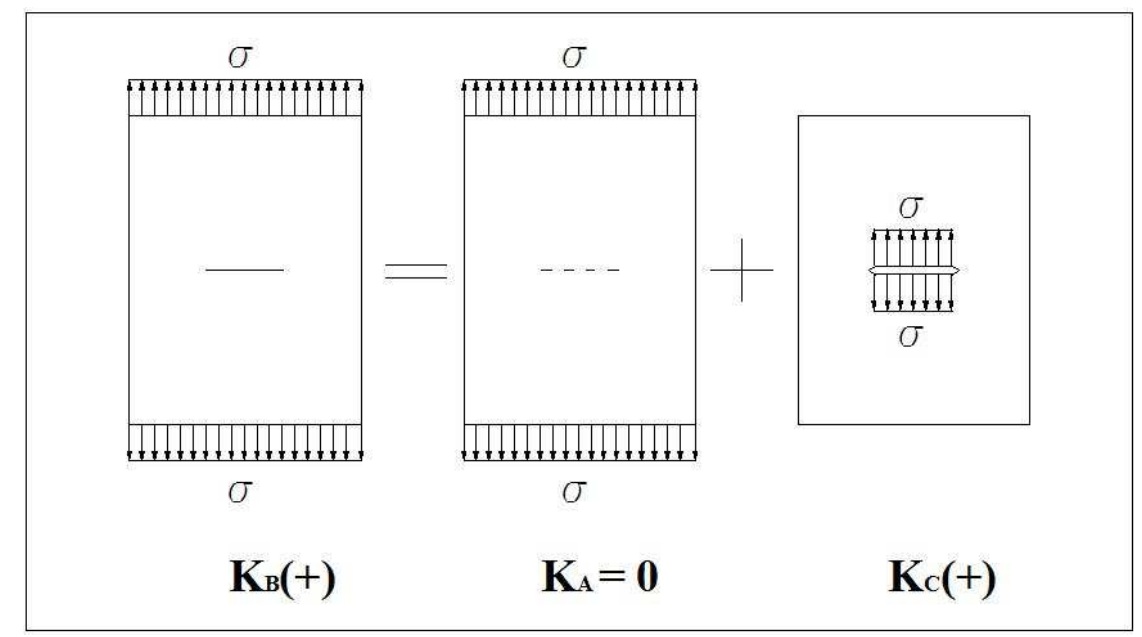

Figura 3.5 - Método da Partição para uma fissura baseado no Princípio de Sobreposição e Princípio de Bueckner

Tendo em vista a figura 3.5, observam-se, então, as bases do Método da Partição: uma decomposição consistindo em um subdomínio ausente de fissuras $P_{G}^{(0)}$ e outro contendo a fissura $P_{L}^{(k)}$. Extrapolando a aplicação dos princípios para o caso de sólido com duas fissuras de borda, tem-se a seguinte configuração descrita na figura 3.6:

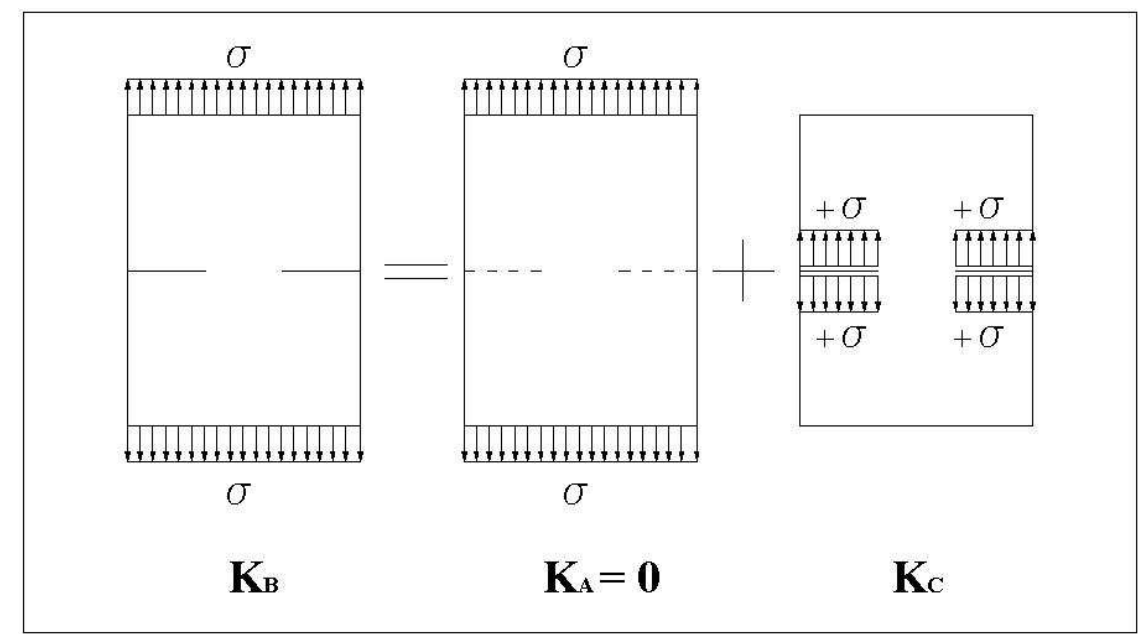

Figura 3.6 - Método da Partição baseado na extrapolação do Princípio de Sobreposição e Princípio de Bueckner para duas fissuras 
Observando-se o segundo subproblema $\left(K_{C}\right)$ na figura anterior, é aparente que as fissuras possam agir uma sobre a outra, influenciando-se mutuamente com reflexos sobre suas aberturas e nos valores dos fatores de intensidade de tensão. Neste caso em particular, nota-se que uma fissura tende a induzir a outra a fechar-se. Isto seria mais facilmente compreendido na ausência de simetria, por exemplo, se uma fissura fosse bem maior que a outra, desta maneira, a abertura da maior fissura exerceria um efeito de fechamento sobre a menor com suas faces comprimidas uma sobre a outra.

Procurando reproduzir a singularidade local do campo de tensão junto à ponta de cada fissura e a interação entre fissuras, o segundo subproblema pode ser dividido em dois outros Andersson, Babuška e Stehlin (1998). O primeiro constituindo-se em um subproblema contendo uma fissura padrão inserida em um meio infinito. Neste subproblema determina-se ao longo de uma linha arbitrária $\Gamma^{(i)}$ o campo de deslocamento. O segundo procura levar em conta a influência desta fissura nas demais. No domínio global sem fissuras aplica-se o campo de deslocamentos na mesma linha $\Gamma^{(i)}$, determinando-se a distribuição de tensão ao longo da linha de fissura externa ao contorno $\Gamma^{(i)}$. A figura 3.7 ilustra a sobreposição:

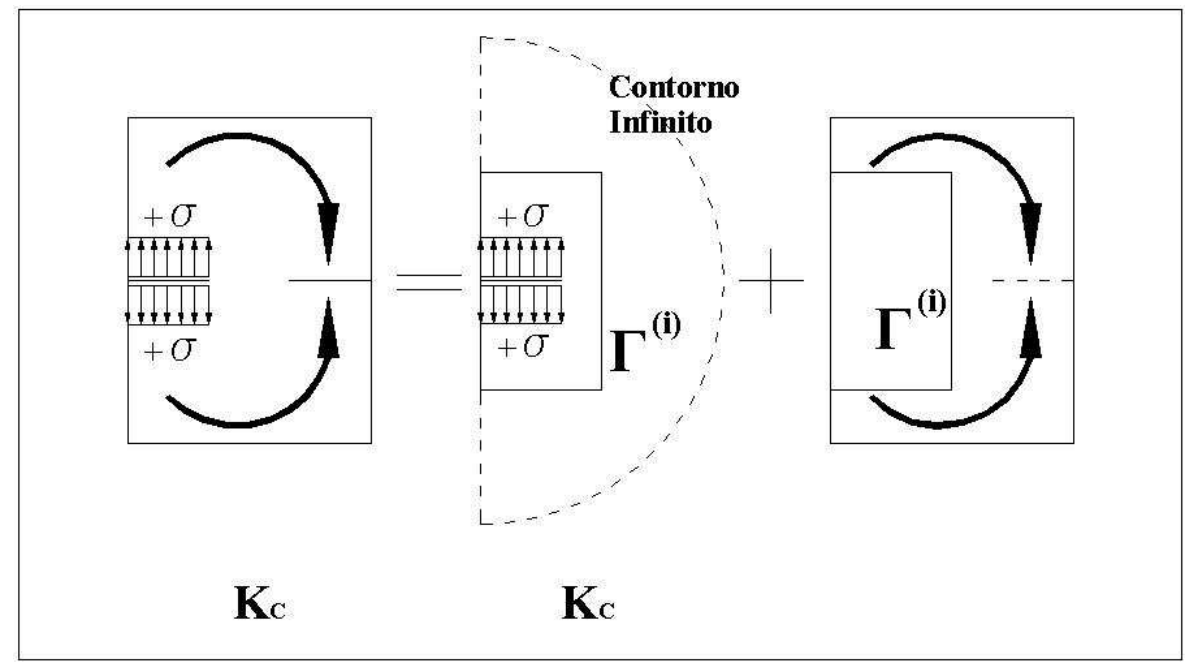

Figura 3.7 - Divisão do segundo subproblema do Princípio de Sobreposição 


\subsection{O Método da Partição}

Andersson, Babuška e Stehlin (1998) introduzem o Método da Partição para a análise de múltiplas fissuras em problemas de engenharia, como sendo um método de resposta confiável e precisa, além de baixo custo computacional no confronto com métodos similares. A motivação para este tipo de análise são os acidentes em aeronaves associados a rupturas de carenagens decorrentes da interação entre múltiplas fissuras que se localizam, em especial, nas regiões de rebites.

O referido método baseia-se na resolução de problemas-padrões (fissura contida em meio infinito) cujas soluções, multiplicadas por fatores de escala, proporcionarão a solução final para a determinação dos fatores de intensidade de tensão. Outro princípio utilizado pelo método refere-se ao Princípio de Bueckner que define o fator de intensidade de tensão oriundo da aplicação de uma força remota, como sendo idêntico àquele obtido através da aplicação de um campo de tensões nas faces da fissura semelhante àquele gerado por esta mesma força externa aplicada (em sólido semelhante, porém ausente de fissura).

O problema básico do Método da Partição está representado na Figura 3.8 e consiste num sólido contendo certo número de vazios e fissuras (uma por vazio, mas poderia ser mais) submetido a forças distribuídas $\sigma$ aplicadas na superfície $S$ de contorno. Na Figura 3.8, $S_{i}, S_{c i}$ e $a_{i}$ referem-se, respectivamente, às superfícies do contorno do vazio e fissura $i$ e ao comprimento da fissura $i$.

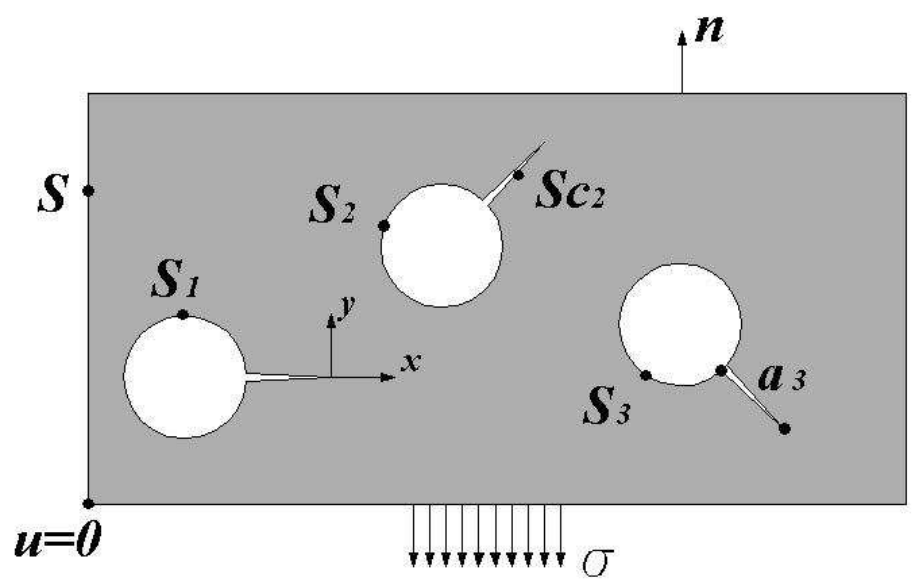

Figura 3.8 - Exemplo de um sólido com múltiplas fissuras 
Um aspecto importante em relação a problemas deste gênero é que a solução nas vizinhanças de cada uma das fissuras é composta de uma parcela regular e outra irregular, governada por um coeficiente que se relaciona com o fator de intensidade de tensão. Tendose em vista esse aspecto e com o objetivo principal de determinar os fatores de intensidade de tensão em cada fissura, o método propõe resolver o problema básico, simbolizado por $P_{G}$, dito global, mediante a combinação das soluções de um subproblema $P_{G}^{(0)}$ que reproduz a parcela regular da solução e dois subproblemas designados $P_{L}^{(k)}$ e $P_{G}^{(k)}$, que reproduzem a parte irregular da solução, (ver Figura 3.9). O valor máximo do índice $k$ (aqui representado por $M$ ) é definido em função dos números $(I)$ de fissuras consideradas e $(J)$ de termos da combinação linear adotados para construir em $P_{G}^{(0)}$ a aproximação dos campos de tensão nas linhas das fissuras, de tal modo que: $k_{\operatorname{máx}}=I \cdot J=M$.

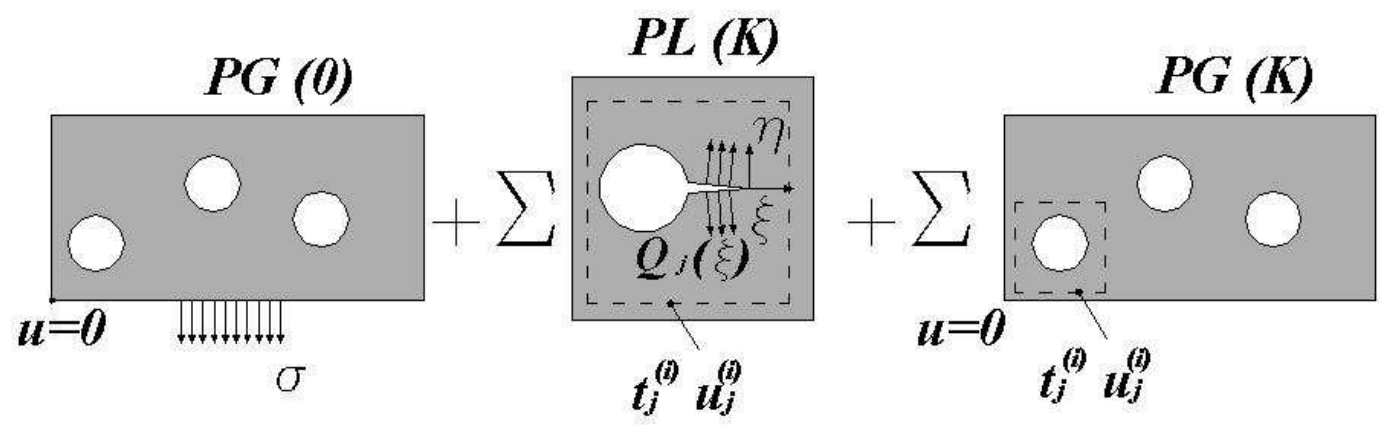

Figura 3.9 - Subproblemas segundo o Método da Partição

A sobreposição dos subproblemas pode ser expressa da seguinte forma:

$P_{G}=P_{G}^{(0)}+\sum_{k=1}^{M} \alpha_{k} \cdot P_{L}^{(k)}+\sum_{k=1}^{M} \alpha_{k} \cdot P_{G}^{(k)}$

Uma vez resolvidos todos os subproblemas, os fatores de escala $\alpha_{k}$ que ditam a solução do problema têm, por sua vez, seus valores calculados a partir da imposição em forma fraca da condição de vetores de tensão nulos nas faces de cada uma das fissuras $(i)$. 


\subsection{Os Subproblemas do Método da Partição}

\subsubsection{O Subproblema Global $P_{G}^{(0)}$}

Como ilustra a figura 3.10, o subproblema $P_{G}^{(0)}$ corresponde ao sólido com mesma geometria, vinculação e forças aplicadas, do problema original $P_{G}$, porém sem a consideração das fissuras. A resposta deste subproblema representa a parte regular da solução geral. Um resultado importante deste subproblema são os vetores de tensão ao longo das linhas (cujas direções e comprimentos são previamente conhecidos) correspondentes às fissuras desconsideradas. Como parte da estratégia do Método da Partição, numa etapa seguinte as distribuições dos vetores de tensão determinadas ao longo das linhas das fissuras são aproximadas por distribuições contínuas, mediante combinações lineares de uma base de funções de aproximação; quanto maior o número adotado $(J)$ de termos da base, melhor a representatividade da aproximação resultante.

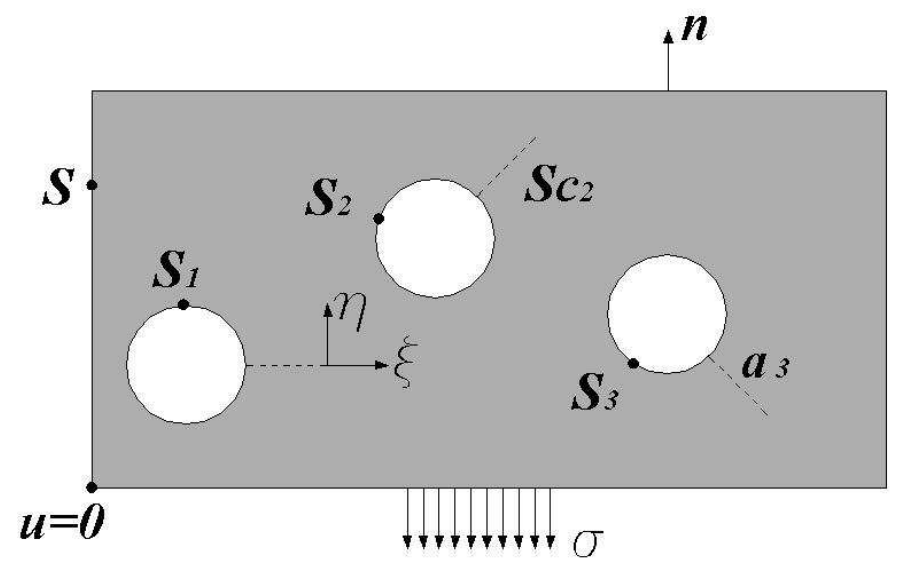

Figura 3.10 - Subproblema $P_{G}^{(0)}$

Dentre as características deste subproblema destacam-se:

a) As variáveis deste subproblema são:

$\checkmark$ Domínio sem a presença de fissuras: $V$

$\checkmark$ Linha de contorno $S$ do domínio de $V$

$\checkmark$ Linha $S_{c i}$ da fissura $i$ 
$\checkmark$ Linha $S_{i}$ do furo $i$

$\checkmark$ Sistema cartesiano de Coordenadas Locais $\xi_{i}, \eta_{i}, \varsigma_{i}$ atrelado à linha de fissura e com origem na sua ponta.

$\checkmark$ Normal à referida linha de contorno: $n$

$\checkmark$ Comprimento $a_{i}$ da fissura i (medido segundo sua projeção em relação a um eixo local $\xi_{i}$ )

b) Equação Fundamental do Subproblema: equação de Navier.

c) Deslocamento imposto no contorno $S$.

$\underline{u}=\left\{\begin{array}{l}u_{x} \\ u_{y}\end{array}\right\}=\underline{\bar{u}} \quad$ em $\quad S_{u}$ (parte da linha de contorno com deslocamentos prescritos)

d) Vetor de tensão imposto em ponto do contorno com normal $n$ :

$$
\underline{t}=\left\{\begin{array}{l}
t_{x} \\
t_{y}
\end{array}\right\}=\underline{\underline{T}} \cdot \underline{n}=\underline{\bar{t}} \quad \text { em } \quad S_{t} \text { (parte da linha de contorno com forças prescritas) }
$$

e) Vetor de tensão nulo nos pontos dos contornos dos furos $i$ :

$$
\underline{\underline{T}} \cdot \underline{n}=\underline{0} \quad \text { em } \quad S_{i} \text { (linha de contorno do furo i) }
$$

f) Meta da análise: determinar vetores de tensão nas linhas de fissuras.

\subsubsection{O Subproblema Local $\boldsymbol{P}_{L}^{(k)}$}

Como ilustra a figura 3.11, cada um dos subproblemas locais $P_{L}^{(k)}$ considera um domínio de contorno $S_{0}^{(i)}$ contendo um vazio e fissura correspondente (os mesmos do problema original $P_{G}$ ). Define-se no interior deste domínio uma linha $\Gamma^{(i)}$ arbitrária de referência contendo o conjunto vazio-fissura. Os problemas locais contemplam a parcela irregular da solução representada pela concentração de tensão nas vizinhanças das pontas de 
cada fissura. As faces da fissura $i$ do conjunto genérico de fissuras $(1 \leq i \leq I)$ são submetidas a um conjunto de $J$ vetores prescritos de tensão cujas distribuições componentes são definidas pelas mesmas componentes da base de aproximação adotada para aproximar os vetores de tensão ao longo das linhas das fissuras no subproblema auxiliar $P_{G}^{(0)}$. Entre os resultados de interesse de cada um dos vetores de tensão $j(1 \leq j \leq J)$ aplicados estão: os campos de deslocamentos $u_{j}^{(i)}$ ao longo de uma linha de contorno arbitrária $\Gamma^{(i)}$ envolvendo a fissura, e o fator de intensidade de tensão. Havendo vazio atrelado à fissura, parte da linha de contorno arbitrária pode coincidir com o contorno do vazio. Sendo $J$ o número de termos da base de tensão e $I$ o número de fissuras, resultam $M=J . I$ subproblemas locais a resolver e, portanto: $1 \leq k \leq M$.

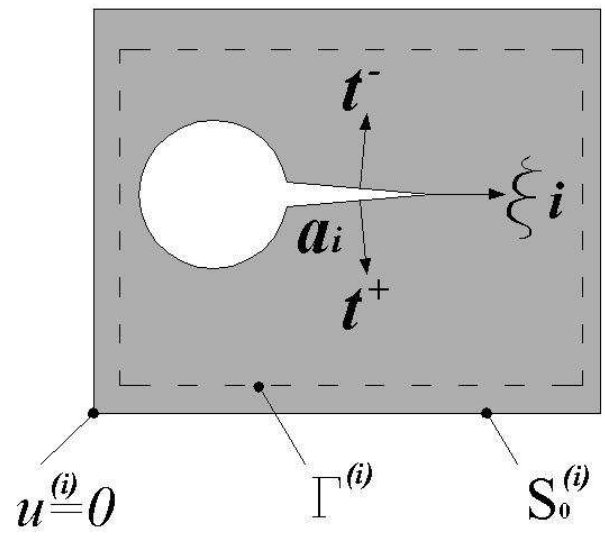

Figura 3.11 - Subproblema $P_{L}^{(k)}$

Entre as características deste subproblema destacam-se:

a) Geometria e variáveis:

$\checkmark$ Domínio local com a presença de fissura: $V_{L}^{(i)}$

$\checkmark$ Linha de contorno do domínio local $V_{L}^{(i)}$ (reproduzindo condições de meio infinito) : $S_{0}^{(i)}$

$\checkmark$ Contorno $S_{c i}$ da fissura $i$ composta por partes $S_{c i}^{+}, S_{c i}^{-}$representando as faces superior e inferior respectivamente.

$\checkmark$ Sistema cartesiano de Coordenadas Locais $\xi_{i}, \eta_{i}, \varsigma_{i}$ com origem na ponta da fissura. 
$\checkmark$ Normal à referida linha de contorno: $n$

$\checkmark$ Comprimento $a_{i}$ da fissura i (medida segundo sua projeção em relação a um eixo local $\xi_{i}$ ).

$\checkmark$ Contorno arbitrário envolvendo o vazio (se existir) e fissura i: ${ }^{(i)} \Gamma$

b) Equação Fundamental do Subproblema: equação de Navier.

c) Deslocamento nulo imposto no contorno $S_{0}^{(i)}$ de $V_{L}^{(i)}$, reproduzindo condições de meio infinito:

$$
\underline{u}=0 \quad \text { em } \quad S_{0}^{(i)}
$$

e) Vetor de tensão nulo no contorno dos furos referentes às fissuras $i$ :

$\underline{t}_{i}=\underline{T} \cdot \underline{n}=\underline{0} \quad$ em $\quad S_{i}$

Vetores de tensão, com distribuição definida pelas componentes da base de aproximação adotada, aplicados nas faces das fissuras i

$$
\begin{aligned}
& \underline{t}^{+}=\underline{Q}_{j}\left(\xi_{i} / a_{i}\right) \quad \text { em } \quad S_{c i}^{+} \\
& \underline{t}^{-}=\underline{Q}_{j}\left(\xi_{i} / a_{i}\right) \quad \text { em } \quad S_{c i}^{-}
\end{aligned}
$$

f) Meta da análise: determinar vetores de deslocamento na linha do contorno arbitrário $\Gamma^{(i)}$ e os fatores de intensidade de tensão para cada subdomínio local e para cada tipo de carregamento aplicado $Q_{j}$.

Importante se faz mencionar a forma de extração dos valores para os fatores de intensidade de tensão. Baseado nos exemplos de Nakasone, Stolarski e Yoshimoto (2006), as seguintes relações foram utilizadas para a determinação dos fatores de intensidade de tensão para os modos I e II de abertura:

$K_{I}=\sqrt{\frac{2 \pi}{r}} \cdot \frac{G}{(\kappa+1)} \cdot C O D$ 


$$
K_{I I}=\sqrt{\frac{2 \pi}{r}} \cdot \frac{G}{(\kappa+1)} \cdot C S D
$$

Sendo:

$r$ : Distância da ponta da fissura até o ponto considerado para a coleta de $C O D$ ou CSD (na configuração indeformada).

G : Módulo de Elasticidade Transversal (ou de cisalhamento), definido como $G=\frac{E}{2(1+v)} \quad, \quad$ onde $E$ é o Módulo de Elasticidade Longitudinal e $v$ é o Coeficiente de Poisson.

$\kappa$ : é igual a $(3-4 v)$ se Estado Plano de Deformação e $\frac{3-v}{1+v}$ se Estado Plano de Tensão.

COD: "Crack Open Displacement" a diferença entre os deslocamentos de pontos correspondentes das faces segundo direção perpendicular à linha de fissura.

CSD: "Crack Sliding Displacement" a diferença entre os deslocamentos de pontos correspondentes das faces segundo direção paralela à linha de fissura.

Com isto, calculam-se os valores de fatores de intensidade de tensão tomando-se pontos próximos à ponta da fissura (até $10 \%$ de $a_{i}$ ) e, posteriormente, para fins de extrapolação, aproxima-se estes valores por uma função linear. $\mathrm{O}$ valor final do fator de intensidade de tensão será aquele definido pela função linear para o ponto correspondente à ponta da fissura, Carvalho (1998) e Bitterncourt et al (2003) e Leonel (2006). Evidentemente, nota-se que, quanto maior a discretização desta região, maior será a tendência dos valores de $K$ formarem uma função linear que extrapolada no sentido da ponta de fissura permitirá a obtenção de um fator de intensidade de tensão com alta precisão. Na figura 3.12 nota-se que é realizada a extrapolação utilizando-se os valores de COD à esquerda da ponta da fissura, sendo que à direita da ponta da fissura, somente a título de exemplo, utilizou-se a extrapolação dos valores de $K$ obtidos através dos valores do campo de tensão próximos à ponta da fissura. 


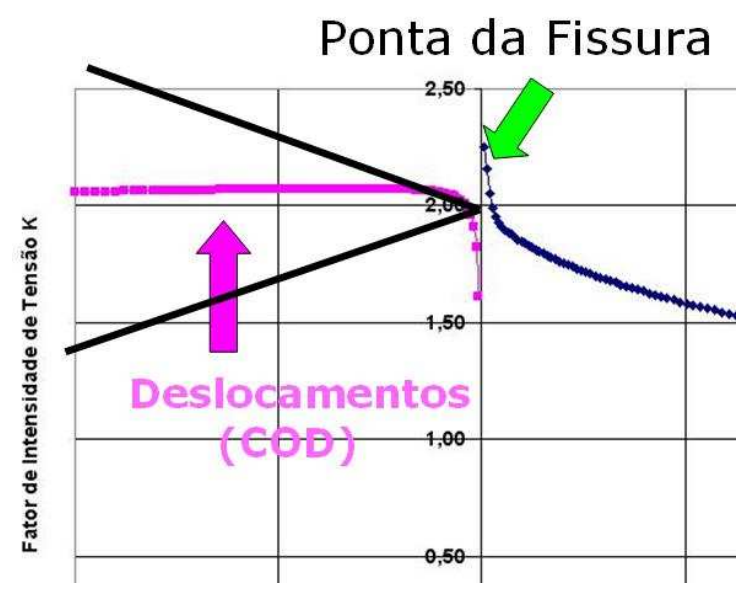

Figura 3.12 - Valores de K calculados em 10\% da face da fissura

Uma última observação sobre este subproblema refere-se ao contorno $\boldsymbol{S}_{\mathbf{0}}^{(i)}$ para a simulação do contorno infinito. Como seu objetivo é representar um problema local que envolva a presença de fissura, quanto mais afastado esse contorno for da fissura menor a sua influência sobre a determinação do fator de intensidade de tensão. Se o comprimento de fissura for muito menor do que as dimensões do sólido considerado, o posicionamento do contorno $S_{0}^{(i)}$ não apresenta dificuldade. Porém, se as dimensões da fissura forem próximas às daquelas que compõem o sólido, o mais viável seria a simulação deste contorno infinito com dimensões e condições de contorno em deslocamentos idênticas ao do problema original (global).

\subsubsection{O Subproblema Global $P_{G}^{(k)}$}

Por último, o subproblema global $P_{G}^{(k)}$ permite reproduzir a interação entre fissuras quando houver um conjunto delas. Em cada um destes subproblemas, conforme ilustra a figura 3.13, considera-se o mesmo domínio existente no subproblema $P_{G}^{(0)}$ (sem fissuras) e com condições de contorno homogêneas (tensão e deslocamentos nulos em $S_{u}$ e $S_{t}$ ). Entretanto, aplicam-se solicitações provenientes do subproblema local $P_{L}^{(k)}$ determinadas, naquele problema, na região do contorno arbitrário $\Gamma^{(i)}$. Em particular, aplicam-se como solicitações os vetores de deslocamento. Observa-se que, ao se compor a solução global por 
sobreposição, recupera-se a continuidade nas distribuições de esforços e deslocamentos, prevalecendo a solução do subproblema $P_{G}^{(0)}$ ao longo das linhas $\Gamma^{(i)}$. Os resultados de interesse de cada subproblema $P_{G}^{(k)}$ são os vetores de tensão nas linhas das fissuras vizinhas influenciadas pela fissura $\boldsymbol{k}$ considerada, ou seja, para cada subproblema $\boldsymbol{k}$ de determinada fissura $i$, coletam-se os valores de tensão nas linhas de fissura externas ao contorno $\Gamma^{(i)}$ que envolve esta mesma fissura $\boldsymbol{i}$. Sendo estes vetores de tensão aproximados usando a mesma base, e número de componentes, adotada no subproblema $P_{G}^{(0)}$.

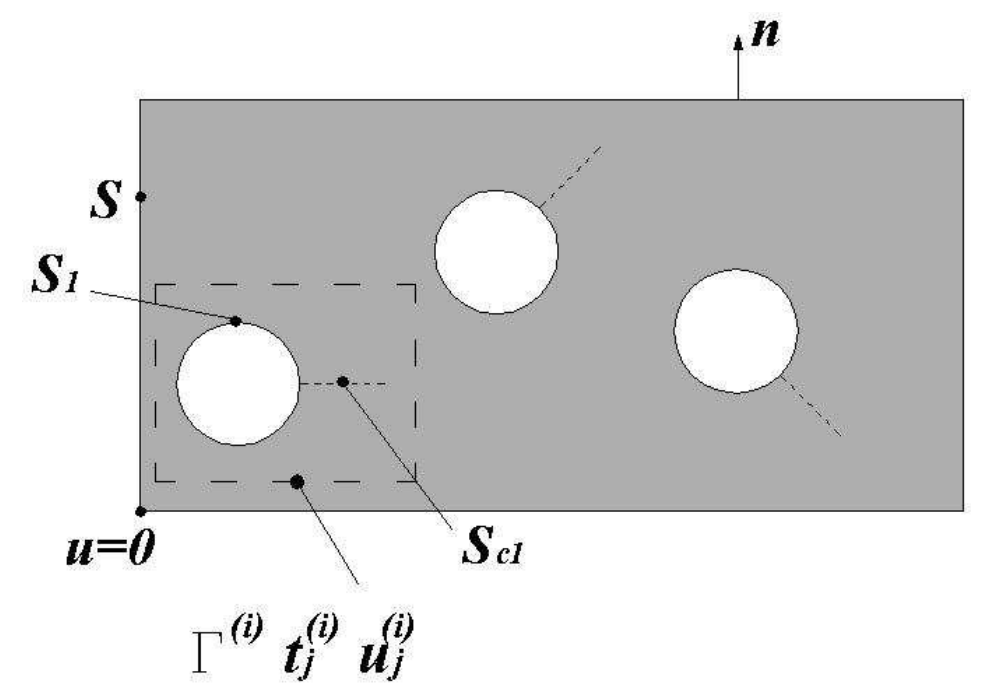

Figura 3.13 - Subproblema $P_{G}^{(k)}$

Entre as características deste subproblema, destacam-se:

a) Geometria:

$\checkmark$ Domínio sem a presença de fissuras: $V$

$\checkmark$ Linha de contorno de $V: S$

$\checkmark$ Linha de fissura $i: S_{c i}$

$\checkmark$ Sistema cartesiano de Coordenadas Locais $\xi_{i}, \eta_{i}, \varsigma_{i}$ com origem na linha de fissura (referente à ponta no subproblema local)

$\checkmark$ Normal à referida linha de contorno: $n$

$\checkmark$ Comprimento da fissura i (medida segundo sua projeção em relação a um eixo local $\left.\xi_{i}\right): a_{i}$ 
b) Equação Fundamental do Subproblema: equação de Navier em $V$.

c) Deslocamento impostos:

$$
\begin{array}{llll}
\underline{u}=\bar{u} & \text { em } & S_{u} \text { de } S . & \text { (parte do contorno do sólido) } \\
\underline{u}=\underline{u}_{k}^{(i)} & \text { em } & \Gamma^{(i)} . &
\end{array}
$$

d) Vetor de tensão nulo imposto no contorno $S$ :

$$
\underline{\underline{T}} \cdot \underline{n}=\underline{\bar{t}}=0 \quad \text { em } \quad S_{t}
$$

e) Vetor de tensão nulo no contorno dos furos referentes às linhas de fissuras $i$ :

$$
\underline{\underline{T}} \cdot \underline{n}=\underline{0} \quad \text { em } \quad S_{i}
$$

f) Meta da análise: determinação dos vetores de tensão nas linhas previamente identificadas das fissuras. Importante ressaltar que para deslocamentos impostos no contorno $\Gamma^{(i)}$ não é necessária a determinação dos valores de tensão junto à linha da própria fissura deste subdomínio local, uma vez que o objetivo refere-se à determinação da influência da fissura sobre as outras e não em si mesma.

\subsection{A Solução Numérica do Método}

A solução para o problema $P_{G}$ é tal que nas faces de cada uma das fissuras de comprimento $a_{i}$ o vetor de tensão $\underline{t}$ deve resultar nulo. Para a fissura $i$ em forma ponderada ou fraca esta condição pode ser escrita como:

$$
\int_{0}^{a_{i}} \underline{t} \underline{Q}_{j 2}\left(\xi_{i} / a_{i}\right) d \xi=0 \quad c / j 2=1, \ldots, J
$$


Sendo:

$\underline{t}=\underline{t}_{G}^{(0)}+\underline{t}_{L}^{(k)}+\underline{t}_{G}^{(k)}$

$J \quad \mathrm{n}^{\mathrm{o}}$ de termos da aproximação adotados

$\underline{Q}\left(\xi_{i} / a_{i}\right)$ é a função ponderadora definida pelas componentes da base de aproximação adotada para aproximar os campos de tensão aplicados nas faces das fissuras $\boldsymbol{i}$. Os fluxos de tensões de cada subproblema na equação (3.6) também envolvem a base de aproximação adotada:

$\underline{t}_{G}^{(0)} \approx \sum_{j=1}^{J} b_{i, j} \cdot \underline{Q}_{j}\left(\xi_{i} / a_{i}\right) \quad c / \quad 0 \leq \xi_{i} \leq a_{i}$

$\underline{t}_{L}^{(k)}=-\sum_{j=1}^{J} \alpha_{j+(i-1) J} \cdot \underline{Q}_{j}\left(\xi_{i} / a_{i}\right) \quad c / \quad 0 \leq \xi_{i} \leq a_{i}$

$\underline{t}_{G}^{(k)}=\sum_{j=1}^{J} \sum_{k=1}^{M} \alpha_{k} \cdot c_{i, j}^{(k)} \cdot \underline{Q}_{j}\left(\xi_{i} / a_{i}\right) \quad c / \quad 0 \leq \xi_{i} \leq a_{i}$

Adicionando as três equações acima conforme exige a equação (3.7) e posteriormente substituindo-se em (3.6), tem-se que:

$$
\begin{aligned}
& \int_{0}^{a_{i}}\left[\sum_{j=1}^{J} b_{i, j} \cdot \underline{Q}_{j}\left(\xi_{i} / a_{i}\right)-\sum_{j=1}^{J} \alpha_{j+(i-1) J} \cdot \underline{Q}_{j}\left(\xi_{i} / a_{i}\right)+\sum_{j=1}^{J} \sum_{k=1}^{M} \alpha_{k} \cdot c_{i, j}^{(k)} \cdot \underline{Q}_{j}\left(\xi_{i} / a_{i}\right)\right]^{T} \underline{Q}_{j 2}\left(\xi_{i} / a_{i}\right) d \xi=0 \\
& \Rightarrow \quad \int_{0}^{a_{i}} \sum_{j=1}^{J}\left[b_{i, j}-\alpha_{j+(i-1) J}+\sum_{k=1}^{M} \alpha_{k} \cdot c_{i, j}^{(k)}\right]^{T} \underline{Q}_{j}\left(\xi_{i} / a_{i}\right) \cdot \underline{Q}_{j 2}\left(\xi_{i} / a_{i}\right) d \xi=0 \quad \mathrm{c} / \quad j, j 2=1, \ldots, J
\end{aligned}
$$

Nota-se que $M=I . J$ e os coeficientes das combinações $b_{i, j}$ e $c_{i, j}^{(k)}$ são identificados previamente a partir das soluções dos subproblemas $P_{G}^{(0)}$ e $P_{G}^{(k)}$ respectivamente. Isolando-se o termo $\alpha$ da equação (3.11) chega-se ao sistema linear: 
$[I G] \cdot\{\alpha\}=\{r\}$

Onde $[I G]$ é a matriz de "influência geral", reunindo os termos que definem a interação entre as fissuras. $\mathrm{O}$ vetor solução $\{\alpha\}$ define os fatores de escala $\alpha$ empregados também na determinação dos fatores de intensidade de tensão para cada fissura, conforme indica a relação seguinte:

$K^{(i)}(J)=\sum_{j=1}^{J} \alpha_{j+(i-1) \cdot J} \cdot K_{j}^{(i)}$

onde $K_{j}^{(i)}$ foram calculados nos subproblemas locais $P_{L}^{(k)}$.

Mais detalhes sobre o sistema mencionado por (3.12), assim como a determinação dos termos $\alpha$ para diferentes casos de interesse são dados a seguir.

\subsubsection{O Sistema Linear $[I G] \cdot\{\alpha\}=\{r\}$ para Uma Fissura}

Dada a seguinte equação que define a condição essencial do Método da Partição, expressando a nulidade das tensões ao longo da linha da fissura $i$ :

$$
\int_{0}^{a i} \sum_{j=1}^{J}\left[b_{i, j}-\alpha_{j+(i-1) J}+\sum_{k=1}^{M} \alpha_{k} \cdot c_{i, j}^{(k)}\right] Q_{j}\left(\xi_{i} / a_{i}\right) \cdot Q_{j 2}\left(\xi_{i} / a_{i}\right) d \xi=0 \quad \mathrm{c} / \quad j, j 2=1, \ldots, J
$$

e considerando-se apenas uma única fissura $i=I=1$ e $m$ termos de aproximação $(J=m)$ tem-se que:

$\sum_{k=1}^{M} \alpha_{k} \cdot c_{i, j}^{(k)} \cdot Q_{j}\left(\xi_{i} / a_{i}\right)=0 \quad$ (não há interação entre fissuras) 


$$
\int_{0}^{a i} \sum_{j=1}^{m}\left[b_{1, j}+\alpha_{j+(1-1) m}\right] Q_{j}\left(\xi_{1} / a_{1}\right) \cdot Q_{j 2}\left(\xi_{1} / a_{1}\right) d \xi=0 \quad \text { c/ } \quad j, j 2=1, \ldots, m
$$

Desenvolvendo a equação acima, obtêm-se o seguinte sistema linear:

$[I G] \cdot\{\alpha\}=\{r\}$

Ou seja:

$$
\left[\begin{array}{cccc}
\int_{0}^{a i}-Q_{1} Q_{1} \partial \xi & \int_{0}^{a i}-Q_{2} Q_{1} \partial \xi & \ldots & \int_{0}^{a i}-Q_{m} Q_{1} \partial \xi \\
\int_{0}^{a i}-Q_{1} Q_{2} \partial \xi & \int_{0}^{a i}-Q_{2} Q_{2} \partial \xi & \cdots & \int_{0}^{a i}-Q_{m} Q_{2} \partial \xi \\
\vdots & \vdots & \ddots & \vdots \\
\int_{0}^{a i}-Q_{1} Q_{m} \partial \xi & \int_{0}^{a i}-Q_{2} Q_{m} \partial \xi & \ldots & \int_{0}^{a i}-Q_{m} Q_{m} \partial \xi
\end{array}\right] \cdot\left\{\begin{array}{c}
\alpha_{1} \\
\alpha_{2} \\
\vdots \\
\alpha_{m}
\end{array}\right\}=\left\{\begin{array}{c}
-\int_{0}^{a i}\left(b_{1,1} Q_{1} Q_{1}+b_{1,2} Q_{2} Q_{1}+\ldots+b_{1, m} Q_{m} Q_{1}\right) \partial \xi \\
\vdots i \\
-\int_{0}^{a i}\left(b_{1,1} Q_{1} Q_{2}+b_{1,2} Q_{2} Q_{2}+\ldots+b_{1, m} Q_{m} Q_{2}\right) \partial \xi \\
\vdots \\
-\int_{0}^{a i}\left(b_{1,1} Q_{1} Q_{m}+b_{1,2} Q_{2} Q_{m}+\ldots+b_{1, m} Q_{m} Q_{m}\right) \partial \xi
\end{array}\right\}
$$

Este sistema é utilizado somente para a determinação do fator de intensidade de tensão para problemas contendo uma única fissura, porém aproximado por $J$ termos.

\subsubsection{O Sistema Linear $[I G] \cdot\{\alpha\}=\{r\}$ Para Várias Fissuras}

Neste caso considera-se então a equação do Método da Partição que define a condição de nulidade das tensões na linha da fissura $i$ :

$$
\int_{0}^{a i} \sum_{j=1}^{J}\left[b_{i, j}-\alpha_{j+(i-1) J}+\sum_{k=1}^{M} \alpha_{k} \cdot c_{i, j}^{(k)}\right] Q_{j}\left(\xi_{i} / a_{i}\right) \cdot Q_{j 2}\left(\xi_{i} / a_{i}\right) d \xi=0 \quad \text { c/ } \quad j, j 2=1, \ldots, J
$$


Para I fissuras, tem-se o seguinte conjunto de equações:

$$
\begin{aligned}
& \int_{0}^{a 1} \sum_{j=1}^{J}\left[b_{1, j}-\alpha_{j}+\sum_{k=1}^{M} \alpha_{k} \cdot c_{1, j}^{(k)}\right] Q_{j}\left(\xi_{1} / a_{1}\right) \cdot Q_{j 2}\left(\xi_{1} / a_{1}\right) d \xi=0 \quad \text { cl } \quad j, j 2=1, \ldots, J \\
& \int_{0}^{a 2} \sum_{j=1}^{J}\left[b_{2, j}-\alpha_{j+J}+\sum_{k=1}^{M} \alpha_{k} \cdot c_{2, j}^{(k)}\right] Q_{j}\left(\xi_{2} / a_{2}\right) \cdot Q_{j 2}\left(\xi_{2} / a_{2}\right) d \xi=0 \quad \mathrm{c} / \quad j, j 2=1, \ldots, J \\
& \int_{0}^{a I} \sum_{j=1}^{J}\left[b_{I, j}-\alpha_{j+(I-1) J}+\sum_{k=1}^{M} \alpha_{k} \cdot c_{I, j}^{(k)}\right] Q_{j}\left(\xi_{I} / a_{I}\right) \cdot Q_{j 2}\left(\xi_{I} / a_{I}\right) d \xi=0 \quad \mathrm{c} / \quad j, j 2=1, \ldots, J
\end{aligned}
$$

Adotando-se os seguintes dados:

$$
\begin{array}{llll}
\checkmark & \mathrm{n}^{\mathrm{o}} \text { de termos da aproximação adotados }=m & \Rightarrow & J=m \\
\checkmark & \mathrm{n}^{\mathrm{o}} \text { de fissuras }=\mathrm{n} & \Rightarrow & I=n \\
\checkmark & M=J . I=m . n & &
\end{array}
$$

e tomando por referência a primeira equação, expressa por (3.18), observa-se que esta se desdobra em um novo conjunto de equações, sendo o número de equações deste novo conjunto igual ao número de termos da aproximação $(j 2=1, \ldots, J=1, \ldots, m)$ :

$$
\begin{aligned}
& \int_{0}^{a 1} \sum_{j=1}^{J}\left[b_{1, j}-\alpha_{j}+\sum_{k=1}^{M} \alpha_{k} \cdot c_{1, j}^{(k)}\right] Q_{j}\left(\xi_{1} / a_{1}\right) \cdot Q_{1}\left(\xi_{1} / a_{1}\right) d \xi=0 \quad \mathrm{c} / \quad j=1, \ldots, J \\
& \int_{0}^{a 1} \sum_{j=1}^{J}\left[b_{1, j}-\alpha_{j}+\sum_{k=1}^{M} \alpha_{k} \cdot c_{1, j}^{(k)}\right] Q_{j}\left(\xi_{1} / a_{1}\right) \cdot Q_{2}\left(\xi_{1} / a_{1}\right) d \xi=0 \quad \quad \mathrm{c} / \quad j=1, \ldots, J \\
& \int_{0}^{a 1} \sum_{j=1}^{J}\left[b_{1, j}-\alpha_{j}+\sum_{k=1}^{M} \alpha_{k} \cdot c_{1, j}^{(k)}\right] Q_{j}\left(\xi_{1} / a_{1}\right) \cdot Q_{m}\left(\xi_{1} / a_{1}\right) d \xi=0 \quad \mathrm{c} / \quad j=1, \ldots, J
\end{aligned}
$$

Sendo assim, desenvolvendo todas as equações expressas por (3.18), chega-se ao seguinte sistema linear:

$$
[I G] \cdot\{\alpha\}=\{r\}
$$


onde a matriz $[I G]$ de dimensões $m . n \times$ m.n pode ser decomposta em duas:

$[I G]=[I G]_{L}+[I G]_{G}$

Sendo que $[I G]_{L}$ é uma matriz que possui as integrais responsáveis por descrever as tensões em $P_{L}^{(k)}$ e $[I G]_{G}$ possui as integrais responsáveis pelo efeito de interação entre as fissuras.

A matriz $[I G]_{L}$ que possui $n \times n$ sub-matrizes é dada da seguinte forma:

$[I G]_{L}=\left[\begin{array}{cccc}{\left[Q^{(1)}\right]} & {[0]} & \cdots & {[0]} \\ {[0]} & {\left[Q^{(2)}\right]} & \cdots & {[0]} \\ \vdots & \vdots & \ddots & \vdots \\ {[0]} & {[0]} & \cdots & {\left[Q^{(n)}\right.}\end{array}\right]$

$\therefore$ [0] são sub-matrizes $m \times m$ nulas

$\therefore\left[Q^{(i)}\right]$ são sub-matrizes $m \times m$ para $n$ fissuras $(i=1, \ldots, n)$ :

$$
\left[Q^{(i)}\right]=-\left[\begin{array}{cccc}
\int_{0}^{a i} Q_{1} Q_{1} \partial \xi & \int_{0}^{a i} Q_{2} Q_{1} \partial \xi & \cdots & \int_{0}^{a i} Q_{m} Q_{1} \partial \xi \\
\int_{0}^{a i} Q_{1} Q_{2} \partial \xi & \int_{0}^{a i} Q_{2} Q_{2} \partial \xi & \cdots & \int_{0}^{a i} Q_{m} Q_{1} \partial \xi \\
\vdots & \vdots & \ddots & \vdots \\
\int_{0}^{a i} Q_{1} Q_{m} \partial \xi & \int_{0}^{a i} Q_{1} Q_{m} \partial \xi & \cdots & \int_{0}^{a i} Q_{m} Q_{m} \partial \xi
\end{array}\right]_{m \times m}
$$

Por outro lado, $[I G]_{G}$ que possui $n \times n$ sub-matrizes é dada da seguinte forma:

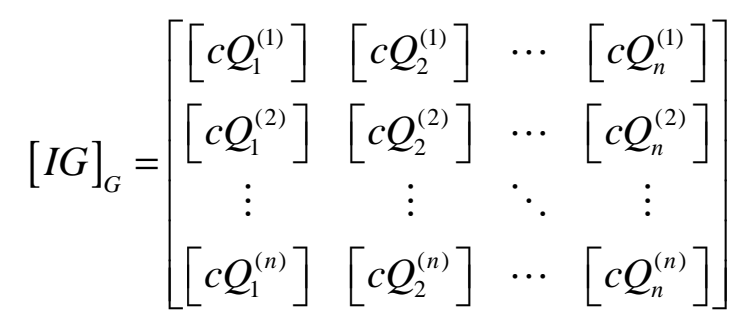


onde $\left[c Q_{i}^{(i)}\right]$ são sub-matrizes $m \times m$ para $n$ fissuras $(i=1, \ldots, n)$ e para $m$ termos de aproximação $(j=1, \ldots, m)$.

Considerando-se as sub-matrizes da primeira linha de $[I G]_{G}$, isto refere-se ao mesmo que considerar-se as influências de todas as fissuras sobre a fissura número 1. Por exemplo, de todas estas sub-matrizes que compõem a linha $1,\left[c Q_{1}^{(1)}\right]$ representa a influência dos carregamentos aproximativos $j$ aplicados na fissura número 1 sobre ela mesma. $\left[c Q_{2}^{(1)}\right]$ representa a influência dos carregamentos aproximativos $j$ aplicados na fissura número 2 sobre a fissura número $1, \mathrm{e}\left[c Q_{n}^{(1)}\right]$ representa a influência dos carregamentos aproximativos $j$ aplicados na fissura número $n$ sobre a fissura número 1 . Desta maneira, a soma de todos os elementos de uma linha da matriz $[I G]_{G}$ formam os $\boldsymbol{k}(=1, \ldots, \mathrm{M})$ subproblemas do Método da Partição.

$$
\begin{aligned}
& {\left[c Q_{1}^{(1)}\right]=\left[\begin{array}{cccc}
\int_{0}^{a i} c_{i, 1}^{(1)} Q_{1} Q_{1}+\ldots+c_{i, m}^{(1)} Q_{m} Q_{1} \partial \xi & \int_{0}^{a i} c_{i, 1}^{(2)} Q_{1} Q_{1}+\ldots+c_{i, m}^{(2)} Q_{m} Q_{1} \partial \xi & \ldots & \int_{0}^{a i} c_{i, 1}^{(m)} Q_{1} Q_{1}+\ldots+c_{i, m}^{(m)} Q_{m} Q_{1} \partial \xi \\
\int_{0}^{a i} c_{i, 1}^{(1)} Q_{1} Q_{2}+\ldots+c_{i, m}^{(1)} Q_{m} Q_{2} \partial \xi & \int_{0}^{a i} c_{i, 1}^{(2)} Q_{1} Q_{2}+\ldots+c_{i, m}^{(2)} Q_{m} Q_{2} \partial \xi & \ldots & \int_{0}^{a i} c_{i, 1}^{(m)} Q_{1} Q_{2}+\ldots+c_{i, m}^{(m)} Q_{m} Q_{2} \partial \xi \\
\vdots & \vdots & \ddots & \vdots \\
\int_{0}^{a i} c_{i, 1}^{(1)} Q_{1} Q_{m}+\ldots+c_{i, m}^{(1)} Q_{m} Q_{m} \partial \xi & \int_{0}^{a i} c_{i, 1}^{(2)} Q_{1} Q_{m}+\ldots+c_{i, m}^{(2)} Q_{m} Q_{m} \partial \xi & \ldots & \int_{0}^{a i} c_{i, 1}^{(m)} Q_{1} Q_{m}+\ldots+c_{i, m}^{(m)} Q_{m} Q_{m} \partial \xi
\end{array}\right]}
\end{aligned}
$$

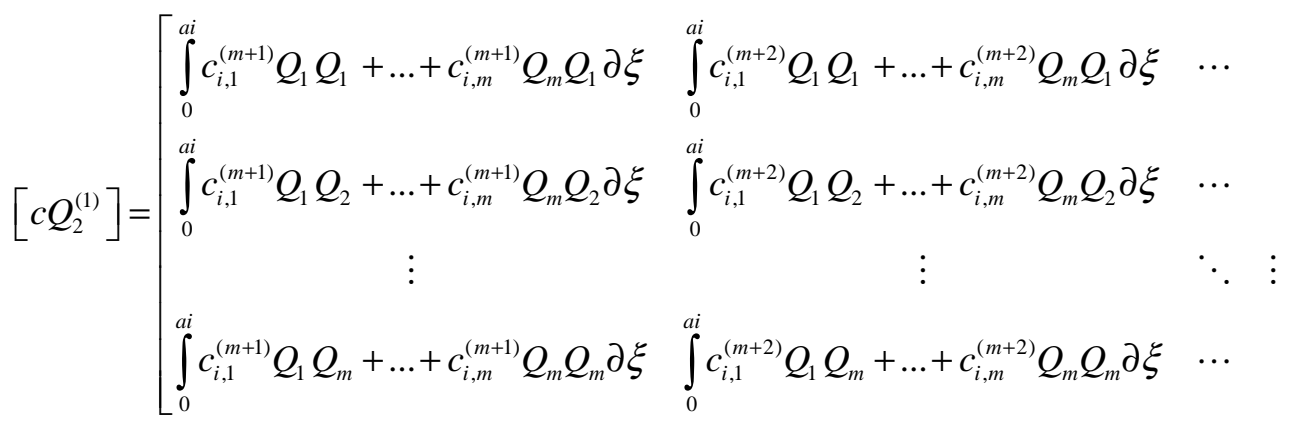

$$
\begin{aligned}
& \left.\ldots \quad \int_{0}^{a i} c_{i, 1}^{(m+m)} Q_{1} Q_{1}+\ldots+c_{i, m}^{(m+m)} Q_{m} Q_{1} \partial \xi\right] \\
& \cdots \quad \int_{0}^{a i} c_{i, 1}^{(m+m)} Q_{1} Q_{2}+\ldots+c_{i, m}^{(m+m)} Q_{m} Q_{2} \partial \xi \\
& \ldots \quad \int_{0}^{a i} c_{i, 1}^{(m+m)} Q_{1} Q_{m}+\ldots+c_{i, m}^{(m+m)} Q_{m} Q_{m} \partial \xi
\end{aligned}
$$




$$
\begin{aligned}
& {\left[c Q_{n}^{(1)}\right]=\left[\begin{array}{ccc}
\int_{0}^{a i} c_{i, 1}^{(k-(1-m))} Q_{1} Q_{1}+\ldots+c_{i, m}^{(k-(1-m))} Q_{m} Q_{1} \partial \xi & \int_{0}^{a i} c_{i, 1}^{(k-(2-m))} Q_{1} Q_{1}+\ldots+c_{i, m}^{(k-(2-m))} Q_{m} Q_{1} \partial \xi & \ldots \\
\int_{0}^{a i} c_{i, 1}^{(k-(1-m))} Q_{1} Q_{2}+\ldots+c_{i, m}^{(k-(1-m))} Q_{m} Q_{2} \partial \xi & \int_{0}^{a i} c_{i, 1}^{(k-(2-m))} Q_{1} Q_{2}+\ldots+c_{i, m}^{(k-(2-m))} Q_{m} Q_{2} \partial \xi & \ldots \\
\vdots & \vdots & \ddots \\
\int_{0}^{a i} c_{i, 1}^{(k-(1-m))} Q_{1} Q_{m}+\ldots+c_{i, m}^{(k-(1-m))} Q_{m} Q_{m} \partial \xi & \int_{0}^{a i} c_{i, 1}^{(k-(2-m))} Q_{1} Q_{m}+\ldots+c_{i, m}^{(k-(2-m))} Q_{m} Q_{m} \partial \xi & \ldots
\end{array}\right.} \\
& \left.\ldots \quad \int_{0}^{a i} c_{i, 1}^{(k)} Q_{1} Q_{1}+\ldots+c_{i, m}^{(k)} Q_{m} Q_{1} \partial \xi\right] \\
& \ldots \quad \int_{0}^{a i} c_{i, 1}^{(k)} Q_{1} Q_{2}+\ldots+c_{i, m}^{(k)} Q_{m} Q_{2} \partial \xi \\
& \left.\cdots \quad \int_{0}^{a i} c_{i, 1}^{(k)} Q_{1} Q_{m}+\ldots+c_{i, m}^{(k)} Q_{m} Q_{m} \partial \xi\right]
\end{aligned}
$$

Importante ressaltar que o objetivo da matriz $[I G]_{G}$ é realizar a interação entre as fissuras. Sendo assim, as sub-matrizes que compõem a diagonal principal desta matriz devem ser eliminadas, uma vez que não tem sentido algum considerar-se o efeito da fissura sobre ela mesma. No caso, isto acarretaria em duplicidade de efeitos uma vez que este efeito já está considerado em $P_{L}^{(k)}$. Outra opção para a não consideração desta duplicidade de efeitos é adicionar o somatório abaixo na equação (3.15):

$$
-\left\{\int_{0}^{a i} \sum_{j=1}^{J}\left[\sum_{l=1}^{J} \alpha_{l+(i-1) J} \cdot c_{i, j}^{[l+(i-1) J]} \cdot Q_{j}\right] Q_{J 2} \partial \xi\right\} \quad \text { c/ } \quad j, j 2=1, \ldots, J
$$

De qualquer modo, a matriz $[I G]_{G}$ deve ser:

$$
[I G]_{G}=\left[\begin{array}{cccc}
{[0]} & {\left[c Q_{2}^{(1)}\right]} & \cdots & {\left[c Q_{n}^{(1)}\right.} \\
{\left[c Q_{1}^{(2)}\right]} & {[0]} & \cdots & {\left[c Q_{n}^{(2)}\right.} \\
\vdots & \vdots & \ddots & \vdots \\
{\left[c Q_{1}^{(n)}\right]} & {\left[c Q_{2}^{(n)}\right]} & \cdots & {[0]}
\end{array}\right]
$$

Definida então a matriz $[I G]$ de dimensão $m . n \times$ m.n resta-se destacar o vetor-solução $\{\alpha\}$ : 
$\{\alpha\}=\left\{\begin{array}{c}\alpha_{1} \\ \alpha_{2} \\ \vdots \\ \alpha_{M}\end{array}\right\}$

assim como o vetor $\{r\}$, responsável pelas integrais das tensões nas linhas de fissuras em $P_{G}^{(0)}$, apresentado da seguinte maneira:

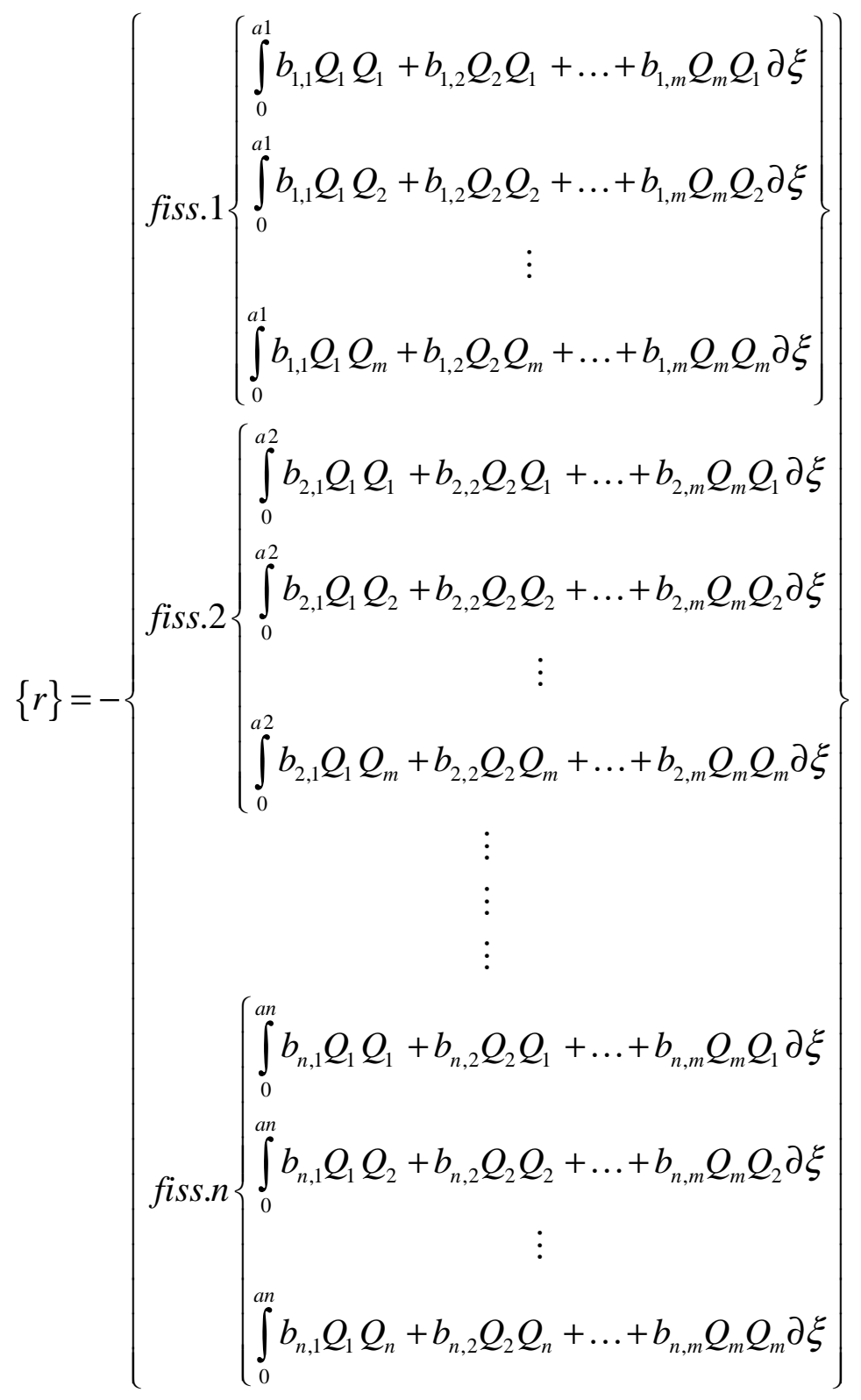




\section{Resultados Numéricos}

Neste capítulo apresentam-se exemplos numéricos do Método da Partição para os modos I e II de abertura. Tais exemplos referem-se a chapas (contendo uma ou mais fissuras) submetidas a carregamentos diversos. Os valores de $\mathrm{K}_{\mathrm{I}}$ e $\mathrm{K}_{\mathrm{II}}$ são as variáveis principais a serem determinadas. Apresenta-se também uma análise do nível de convergência que o Método proporciona para a obtenção de tais valores.

\subsection{Chapas Contendo Fissuras de Borda Solicitadas ao Modo I de Abertura}

\subsubsection{Exemplo 1: Chapa com uma Fissura - Carregamento Uniformemente Distribuído}


Neste caso utiliza-se um exemplo clássico da Mecânica da Fratura com o objetivo de comprovar a eficácia do Método da Partição na obtenção de $\mathrm{K}_{\mathrm{I}}$. Trata-se de uma chapa com dimensões 10x20 ( $\mathrm{W}=10$ e $\mathrm{H}=20$ ), comprimento de fissura a=1, e tensão constante unitária $(\sigma=1)$ aplicada ao longo das faces inferior e superior da chapa, como mostra a figura 4.1:

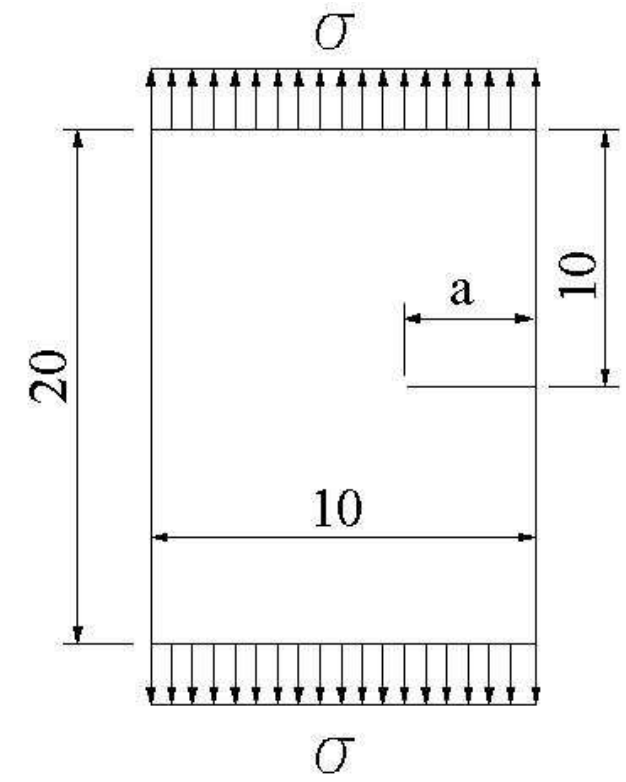

Figura 4.1 - Chapa com 1 fissura e carregamento constante

A solução analítica deste problema deduzida por Ewalds e Wanhill (1986) é dada por:

$K_{I}=C \cdot \sigma \cdot \sqrt{\pi a}$

com:

$C=1,12-0,231\left(\frac{a}{W}\right)+10,55\left(\frac{a}{W}\right)^{2}-21,72\left(\frac{a}{W}\right)^{3}+30,39\left(\frac{a}{W}\right)^{4}$ até $\frac{a}{W}=0,6$

No caso, com as dimensões descritas obtêm-se o valor exato do fator de intensidade de tensão para a fissura:

$\frac{a}{W}=\frac{1}{10}=0,1 \Rightarrow \mathrm{C}=1,183719 \Rightarrow K_{I}=C \cdot \sigma \cdot \sqrt{\pi a}=C \cdot 1 \cdot \sqrt{\pi \cdot 1}=2,0981$ 
Por se tratar de força distribuída uniformemente na chapa, a solução pelo Método da Partição pode ser obtida considerando-se apenas um termo de aproximação. Além disso, como não há interação entre fissuras, a sobreposição se reduz à imposição de resultante nula das tensões na linha de fissura. Neste sentido, aplica-se um carregamento constante de compressão nas faces de fissura de $P_{L}^{(1)}$ para anular as tensões de tração oriundas de $P_{G}^{(0)}$.

Os valores dos índices para a utilização das equações do Método da Partição se reduzem neste caso a:

$\mathrm{I}=1 \quad\left(\mathrm{n}^{\mathrm{o}}\right.$ de fissuras $)$

$\mathrm{J}=1 \quad\left(\mathrm{n}^{\mathrm{o}}\right.$ de termos da aproximação)

$\mathrm{M}=\mathrm{I} . \mathrm{J}=1$

$Q_{j}\left(\xi_{i} / a_{i}\right)=\left(\frac{\xi_{i}}{a_{i}}\right)^{j-1} \quad \Rightarrow \quad Q_{1}\left(\xi_{1} / a_{1}\right)=\left(\frac{\xi_{1}}{a_{1}}\right)^{1-1}=\left(\frac{\xi_{1}}{a_{1}}\right)^{0}=1$

Considerando o subproblema $P_{G}^{(0)}$, tem-se que a ausência de fissura implica em tensão unitária na linha de fissura $\left(\underline{t}_{G}^{(0)}=1\right)$. Sendo assim a tensão na linha de fissura pode ser expressa reduzindo a combinação linear à componente $Q_{1}\left(\xi_{1} / a_{1}\right)$ da base, o que resulta:

$\underline{t}_{G}^{(0)} \approx \sum_{j=1}^{1} b_{1, j} \cdot Q_{1}\left(\xi_{1} / a_{1}\right) \quad \Rightarrow \quad 1=b_{1,1} \cdot 1 \quad \Rightarrow \quad b_{1,1}=1 \quad \Rightarrow \quad \underline{t}_{G}^{(0)} \approx 1$

Como já comentado, o terceiro subproblema $P_{G}^{(k)}$ não necessita ser considerado, pois não há interação entre fissuras.

$\underline{t}_{G}^{(k)}=0$

Aplicando o fluxo de tensão $Q_{1}\left(\xi_{1} / a_{1}\right)=1$ (compressão) na face da fissura, o subproblema local tem seu vetor de tensão representado por: 
$\underline{t}_{-L}^{(k)}=\sum_{j=1}^{J} \alpha_{j+(i-1) J} \cdot Q_{j}\left(\xi_{i} / a_{i}\right) \quad \Rightarrow \quad \underline{t}_{L}^{(1)}=\sum_{j=1}^{1} \alpha_{1+(1-1) 1} \cdot 1 \quad \Rightarrow \quad \underline{t}_{L}^{(1)}=\alpha_{1} \cdot 1$

Com a ajuda do programa ANSYS, pode-se extrair o fator de intensidade de tensão, obtendo-se:

$K_{j}^{(i)}=K_{1}^{(1)}=2,099$

Com todas as informações obtidas e através da equação (3.15), obtêm-se o parâmetro alfa que dará a solução do problema:

$$
\begin{aligned}
& \int_{0}^{a_{i}} \sum_{j=1}^{J}\left[b_{i, j}-\alpha_{j+(i-1) J}+\sum_{k=1}^{M} \alpha_{k} \cdot c_{i, j}^{(k)}\right]^{T} Q_{j}\left(\xi_{i} / a_{i}\right) \cdot Q_{j 2}\left(\xi_{i} / a_{i}\right) d \xi=0 \\
& \Rightarrow \quad \int_{0}^{1} \sum_{j=1}^{1}\left[1-\alpha_{1+(1-1) 1}+0\right] 1 \cdot 1 d \xi=0 \Rightarrow \int_{0}^{1} \sum_{j=1}^{1}\left[1-\alpha_{1}\right] d \xi=0 \Rightarrow \int_{0}^{1}\left(1-\alpha_{1}\right) d \xi=0 \\
& \left.\Rightarrow\left(1-\alpha_{1}\right) \xi\right|_{0} ^{1}=0 \Rightarrow \alpha_{1}=1
\end{aligned}
$$

Sendo assim, obtêm-se o fator de intensidade de tensão do problema global para a fissura $\boldsymbol{i}(=1)$ :

$$
K^{(i)}(J)=\sum_{j=1}^{J} \alpha_{j+(i-1) \cdot J} \cdot K_{j}^{(j)} \Rightarrow K^{(1)}(1)=\sum_{j=1}^{1} \alpha_{1} \cdot K_{1}^{(1)}=\sum_{j=1}^{1} 1 \cdot 2,099=2,099
$$

O erro da aproximação pode ser expresso da seguinte maneira:

$$
E(\%)=\frac{\left|K_{R E F}-K_{A P R O X}\right|}{K_{R E F}} \cdot 100=\frac{|2,0981-2,099|}{2,0981} \cdot 100=0,043 \%
$$

Com o resultado obtido verifica-se a eficiência do Princípio de Bueckner, permitindo redefinir um problema mediante à resolução de dois subproblemas seguido de superposição. No caso, somente o termo constante da base de aproximação do vetor de tensão na linha da fissura foi o suficiente para capturar a solução exata do problema. 


\subsubsection{Exemplo 2: Chapa com uma Fissura - Carregamentos Não-Uniformes}

Os seguintes exemplos têm como objetivo demonstrar a eficácia do Método da Partição na análise de problemas contendo o campo de tensões na linha da fissura do problema $P_{G}^{(0)}$ não-uniforme. Neste caso, este campo é aproximado por uma base de funções polinomiais. Outro objetivo refere-se à obtenção do fator de intensidade de tensão gerado por este mesmo campo de tensões não-uniformes mediante a combinação de outros fatores obtidos em cada subproblema local. Os exemplos contemplam diferentes tipos de carregamentos: linear, quadrático, constante+quadrático mostrando que, quanto mais complexa a distribuição das tensões na linha de fissura, mais termos da aproximação deverão ser adotados. As características geométricas dos exemplos deste item são as mesmas do item anterior. Os exemplos considerados estão representados na figura 4.2.

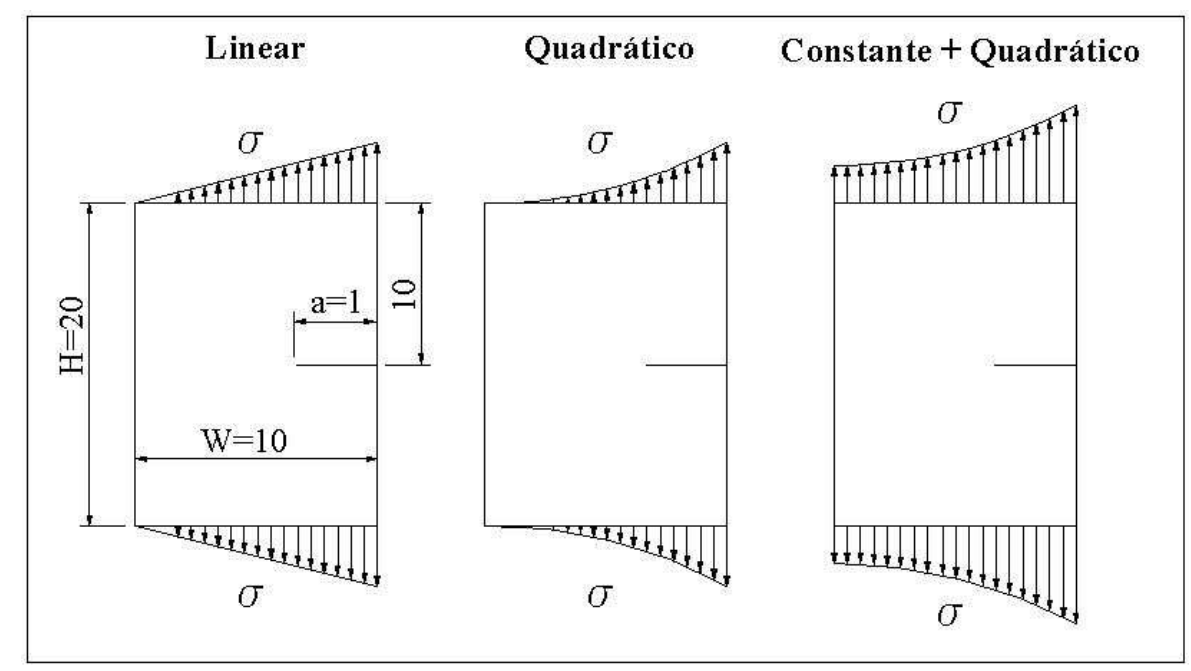

Figura 4.2 - Chapa com 1 fissura e carregamentos variados

Para o emprego do programa ANSYS duas malhas foram geradas, uma malha não refinada para o subproblema $P_{G}^{(0)}$ e outra malha, para o subproblema $P_{L}^{(k)}$, bastante refinada na região próxima à ponta fissura. Os dados referentes ao material e adotados no modelo são indicados em seguida:

$G=1,0$

$v=0,3$ 
Optou-se pela geração de uma malha de $P_{L}^{(k)}$ contendo as seguintes características:

$\checkmark$ Eixo de simetria horizontal

$\checkmark$ Elemento finito quadrilateral de oito nós (deslocamentos em duas direções por nó)

$\checkmark \quad \mathrm{N}^{\mathrm{o}}$ de nós $=9595$

$\checkmark \quad N^{\circ}$ de Elementos $=3136$

$\checkmark$ Refinamento da malha junto à ponta da fissura

$\checkmark$ Malha não-estruturada

Para a extração dos valores dos fatores de intensidade de tensão-referência dos três casos, utilizou-se a técnica de correlação de deslocamentos enunciada no capítulo anterior:

$$
K_{I}=\sqrt{\frac{2 \pi}{r}} \cdot \frac{G}{(\kappa+1)} \cdot C O D
$$

Desta maneira, calculam-se os valores de deslocamentos próximos à ponta da fissura (até $10 \%$ de $a$ ) e obtêm-se os valores de $\mathrm{K}_{\mathrm{I}}$ referência, mediante um processo de extrapolação, obtendo-se os seguintes valores para os três casos mencionados anteriormente:

Tabela 4.1 - Valores-Referência de $K_{I}$ para carregamentos variados

\begin{tabular}{|l|c|}
\hline \multicolumn{1}{|c|}{ Carregamento } & $\mathbf{K}_{\mathbf{I}}$ \\
\hline Linear & 1,986 \\
\hline Quadrático & 1,640 \\
\hline cte + Quadrático & 3,750 \\
\hline
\end{tabular}

Para a resolução do Método da Partição, faz-se necessário aproximar o campo de tensão na linha de fissura mediante combinação linear de uma base polinomial. Na figura 4.3 mostram-se os pontos de referência empregados na construção da aproximação com até três elementos de base polinomial: 


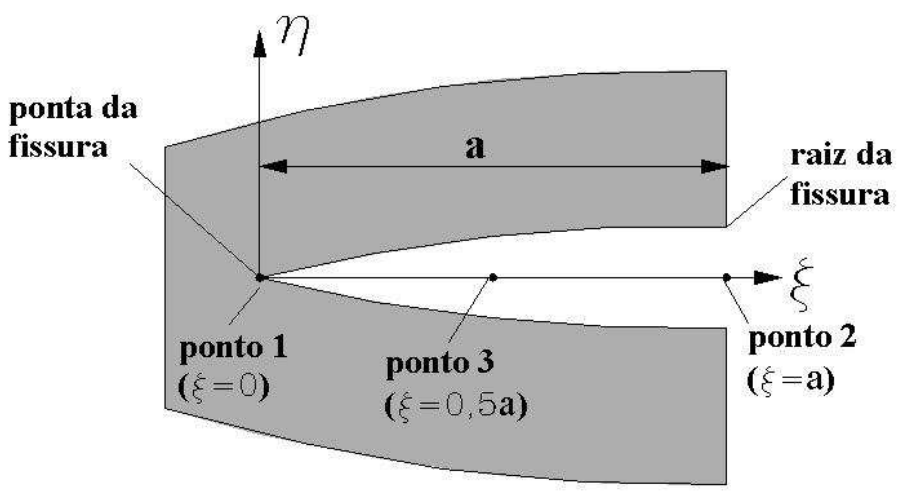

Figura 4.3 - Eixo das coordenadas locais e pontos de coleta das tensões

Sendo assim, para um termo da base de aproximação considerado (constante), os valores de tensão nas linhas de fissura a serem considerados são àqueles referentes ao ponto 1 ; para dois termos da base de aproximação (constante e linear) utilizados considera-se os pontos 1 e 2; e para três termos da aproximação considerados (constante, linear e quadrático), coleta-se os valores dos pontos 1,2 e 3 .

Uma malha estruturada menos refinada foi empregada para o subproblema $P_{G}^{(0)}$ contendo apenas 900 elementos quadráticos de oito nós com dois graus de liberdade nodais (30x30). A malha para o subproblema local $P_{L}^{(k)}$ é a mesma utilizada no processo de extração de $K$. Para cada um dos exemplos o Método da Partição fornece os resultados mostrados nas tabelas $4.2,4.3$ e 4.4 .

Tabela 4.2 - Valores de $K_{I}$ aproximado obtidos para carregamento linear

\begin{tabular}{|c|c|c|}
\hline \multicolumn{3}{|c|}{ Carregamento Linear: $\mathrm{K}_{\mathrm{EX}}=1,986$} \\
\hline $\mathrm{N}^{\mathrm{o}}$ termos Aprox. & $\mathrm{K}_{\mathrm{APROX}}$ & $\mathrm{E}(\%)$ \\
\hline 1 & 1,8999 & 4,34 \\
\hline 2 & 1,9851 & 0,05 \\
\hline
\end{tabular}

Tabela 4.3 - Valores de $\mathrm{K}_{\mathrm{I}}$ aproximado obtidos para carregamento quadrático

\begin{tabular}{|c|c|c|}
\hline \multicolumn{3}{|c|}{ Carregamento Quadrático: $\mathrm{K}_{\mathrm{EX}}=1,640$} \\
\hline $\mathrm{N}^{\circ}$ termos Aprox. & $\mathrm{K}_{\text {APROX }}$ & $\mathrm{E}(\%)$ \\
\hline 1 & 1,5534 & 5,28 \\
\hline 2 & 1,6373 & 0,16 \\
\hline
\end{tabular}


Tabela 4.4 - Valores de $K_{I}$ aproximado obtidos para carregamento cte+quadrático

\begin{tabular}{|c|c|c|}
\hline \multicolumn{3}{|c|}{ Carregamento Cte + Quadrático: $\mathrm{K}_{\mathrm{EX}}=3,750$} \\
\hline $\mathrm{N}^{\mathrm{o}}$ termos Aprox. & $\mathrm{K}_{\mathrm{APROX}}$ & $\mathrm{E}(\%)$ \\
\hline 1 & 3,6645 & 2,28 \\
\hline 2 & 3,7399 & 0,27 \\
\hline 3 & 3,7465 & 0,09 \\
\hline
\end{tabular}

Com os resultados obtidos, fica evidente a capacidade de obtenção dos valores dos fatores de intensidade de tensão finais mediante uma combinação linear de outros casos mais simples (subproblemas locais). Observa-se que, quanto mais complexos são os carregamentos (e por conseqüência os campos de tensão na linha de fissura de $P_{G}^{(0)}$ ), mais termos aproximativos necessitavam ser utilizados para a obtenção de valores mais precisos. Outra característica relevante refere-se à convergência que se mostrou exponencial, ou seja, com poucos termos de aproximação foi possível obter-se resultados com um nível de erro muito baixo.

\subsubsection{Exemplo 3: Chapa com Duas Fissuras - Carregamento Não-Uniforme}

No exemplo deste item, procura-se incluir a interação entre as fissura. A fim de avaliar-se o desempenho do método, toma-se como base o exemplo anterior, considerando-se o carregamento externo do tipo constante+quadrático, porém com uma fissura a mais, conforme mostra a figura 4.4 : 


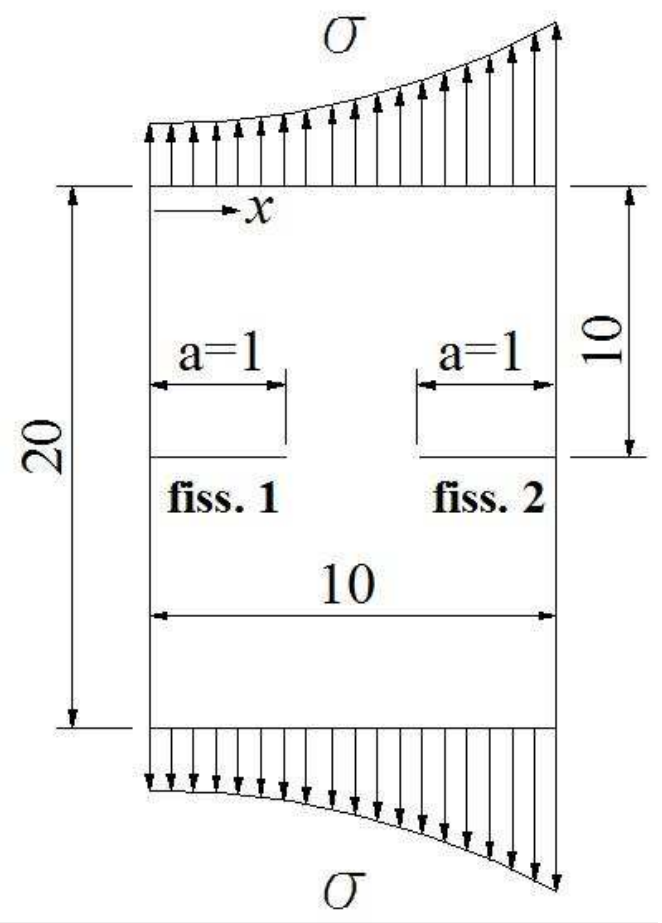

Figura 4.4 - Chapa com 2 fissuras e carregamento cte+quadrático

Os dados do problema são os seguintes:

a) Propriedades do material:

$$
\begin{aligned}
& G=1,0 \\
& v=0,3
\end{aligned}
$$

b) Carregamento:

$$
\sigma_{y}(x)=1+\left(\frac{x}{10}\right)^{2}
$$

Para a determinação dos fatores de intensidade de tensão-referência realiza-se uma simulação com o programa ANSYS com malhas contendo as seguintes características:

$\checkmark$ Eixo de simetria horizontal

$\checkmark$ Elemento finito quadráticos de oito nós (dois graus de liberdade nodais)

$\checkmark \quad \mathrm{N}^{\mathrm{o}}$ de nós $=25107$

$\checkmark \quad \mathrm{N}^{\mathrm{o}}$ de Elementos $=8310$

$\checkmark$ Refinamento da malha junto à ponta da fissura. 
Os fatores de intensidade de tensão-referência para cada fissura resultam com os valores mostrados na tabela 4.5 :

Tabela 4.5 - Valores-Referência de $K_{\mathrm{I}}$ obtidos para carregamento cte+quadrático

\begin{tabular}{|c|c|}
\hline Fissura & $\mathbf{K}_{\mathbf{I}}$ \\
\hline 1 & 1,633 \\
\hline 2 & 3,642 \\
\hline
\end{tabular}

Os valores aproximados dos fatores de intensidade de tensão, para as duas fissuras, obtidos com o Método da Partição estão mostrados na tabela 4.6 :

Tabela 4.6 - Valores de $K_{I}$ aproximados obtidos para carregamento cte+quadrático (2 fissuras)

\begin{tabular}{|c|c|c|c|c|c|}
\hline \multicolumn{5}{|c|}{ Carregamento Cte+Quadrático } \\
\hline \multicolumn{3}{|c|}{ Fissura 1: K } & \multicolumn{3}{c|}{ Fissura 2: $\mathbf{K}_{\mathbf{R E F}}=\mathbf{3 , 6 4 2}$} \\
\hline $\begin{array}{c}\mathbf{N}^{\mathbf{0}} \text { termos } \\
\text { Aprox. }\end{array}$ & $\mathbf{K}_{\text {APROx }}$ & $\mathbf{E}(\%)$ & $\begin{array}{c}\mathbf{N}^{\mathbf{0}} \text { termos } \\
\text { Aprox. }\end{array}$ & $\mathbf{K}_{\text {APROx }}$ & $\mathbf{E}(\%)$ \\
\hline 1 & 1,6683 & 2,16 & 1 & 3,6611 & 0,52 \\
\hline 2 & 1,6286 & 0,27 & 2 & 3,6359 & 0,17 \\
\hline 3 & 1,6322 & 0,05 & 3 & 3,6384 & 0,10 \\
\hline
\end{tabular}

Com os resultados obtidos, confirma-se a capacidade do Método da Partição na obtenção dos fatores de intensidade de tensão para problemas da Mecânica da Fratura envolvendo mais de uma fissura. Novamente, a convergência se mostrou de modo exponencial, sendo igualmente necessários apenas três termos da aproximação para a obtenção de um erro inferior a $0,5 \%$. Devido ambas as fissuras possuírem a mesma forma geométrica (comprimento) e de contorno (borda), adota-se um contorno arbitrário $\Gamma^{(i)}$ idêntico para as mesmas. Desta maneira, ao se resolver os subproblemas locais $P_{L}^{(k)}$ para a 
fissura 1 (esquerda), automaticamente estes resultados servem também para o subproblema local da fissura 2 (da direita) tendo em vista a igualdade dos contornos $\Gamma^{(i)}$ do problema. Conseqüentemente, o subproblema $P_{L}^{(k)}$ necessita somente de uma única malha (contendo refinamento na ponta da fissura), onde se altera somente o carregamento nas faces de fissura (base aproximativa de funções polinomiais - $Q_{j}$ ). Uma vez resolvido o subproblema local $P_{L}^{(k)}$ obtendo-se o vetor de deslocamentos no seu contorno $\Gamma^{(i)}$, seu respectivo subproblema $P_{G}^{(k)}$ é resolvido aplicando-se este mesmo vetor de deslocamentos no contorno $\Gamma^{(i)}$ de $P_{G}^{(k)}$. Note que para a utilização deste vetor de deslocamentos na linha de fissura 1 (da esquerda) de $P_{G}^{(k)}$ não é necessário fazer-se nenhuma rotação do mesmo. Em contrapartida, para a utilização deste vetor na linha de fissura 2 (da direita), faz-se necessário, no caso, utilizar a simetria vertical do problema (ver figura 4.5).

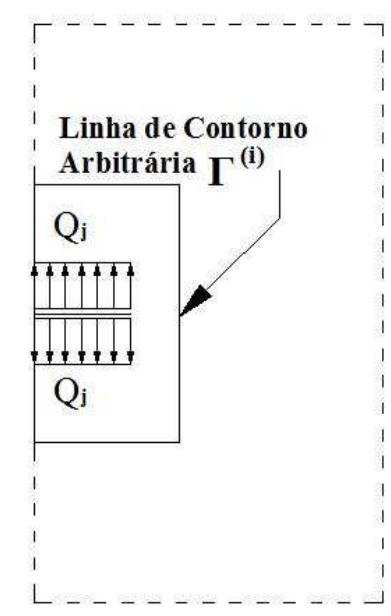

$\mathbf{P}_{\mathbf{L}}^{(\mathbf{k})}$

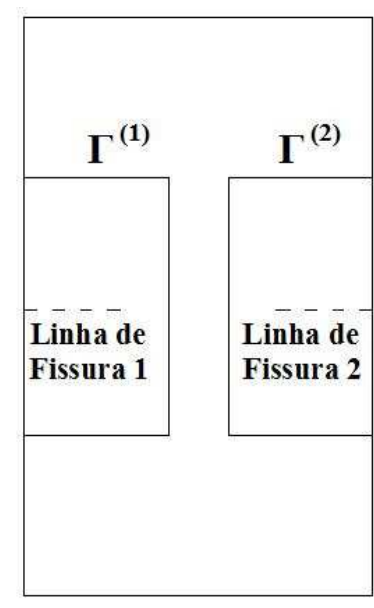

$\mathbf{P}_{\mathbf{G}}^{(\mathbf{k})}$

Figura 4.5 - Definição do contorno $\Gamma^{(i)}$ para as linhas de fissura. 


\subsection{Chapas Contendo Fissuras de Borda Solicitadas ao Modo II de Abertura}

O Método da Partição também se aplica às análises envolvendo fissuras submetidas ao modo II de abertura. Para tais análises os mesmos procedimentos relativos ao modo I de abertura são realizados, porém nos problemas locais os carregamentos incógnitos $Q_{j}$ são aplicados na direção tangente à face da fissura, ou seja, na direção do eixo local $\xi$ da fissura, em sentidos opostos para as duas faces de fissuras, proporcionando um cisalhamento com sinal contrário àquele obtido na linha de fissura do subproblema $P_{G}^{(0)}$.

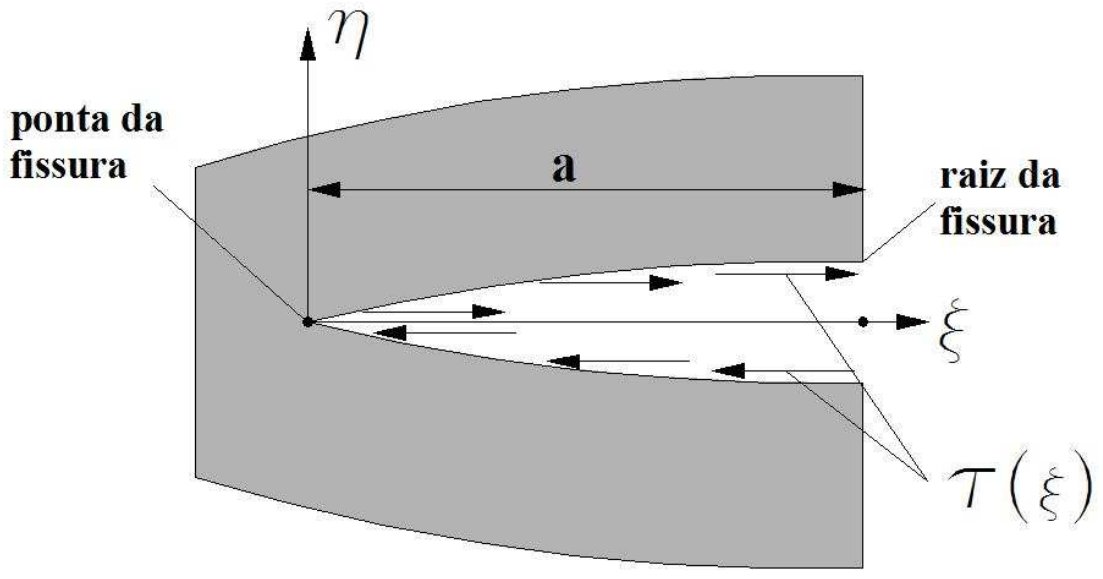

Figura 4.6 - Fluxo de tensões $Q_{j}$ aplicado nas faces da fissura para obtenção de $K_{I I}$

\subsubsection{Exemplo 1: Chapa com Uma Fissura - Carregamento Não-Uniforme}

Neste caso, utiliza-se o Método da Partição para a obtenção de $\mathrm{K}_{\mathrm{II}}$. $\mathrm{O}$ exemplo trata de uma chapa com dimensões 10x20 ( $\mathrm{W}=10$ e $\mathrm{H}=20$ ), comprimento de fissura $\mathrm{a}=1$, carregamento cisalhante $\tau$ com variação quadrática aplicada ao longo da borda superior da chapa. A mesma possui vinculações nas direções x e y na face inferior. 


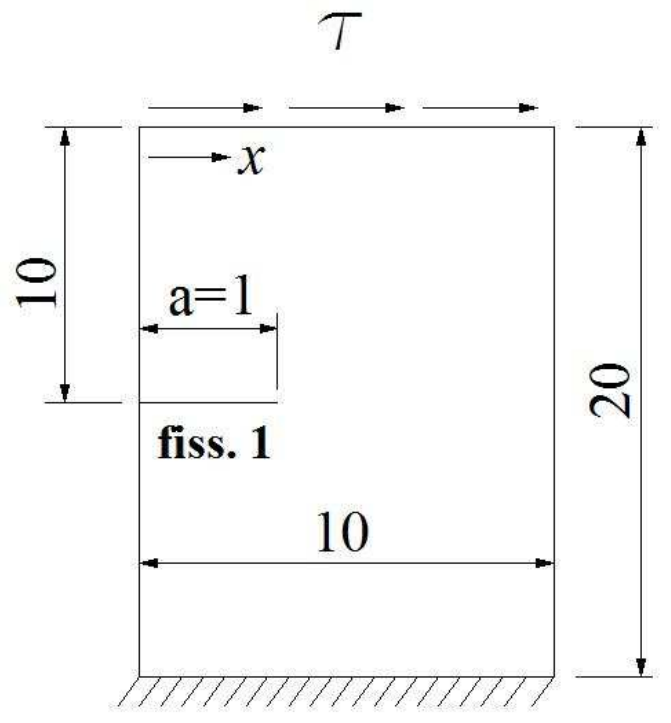

Figura 4.7 - Chapa com 1 fissura e carregamento cte+quadrático cisalhante

O valor referência de $K_{\text {II }}$ para este caso é de 0,877 , sendo o mesmo obtido mediante procedimentos descritos em 3.6.2. Os dados do problema são os seguintes:

a) Propriedades do material:

$G=1,0$

$v=0,3$

b) Carregamento:

$$
\tau(x)=1+\left(\frac{x}{10}\right)^{2}
$$

c) As malhas dos subproblemas locais e globais são as mesmas descritas na análise referente ao modo I de abertura, bem como o tipo de extração dos fatores de intensidade de tensão locais.

Tabela 4.7 - Valores de $K_{\text {II }}$ aproximado obtidos para carregamento cte+quadrático cisalhante

\begin{tabular}{|c|c|c|}
\hline \multicolumn{3}{|c|}{ Carregamento Cte + Quadrático: $\mathrm{K}_{\mathrm{REF}}=0,877$} \\
\hline $\mathrm{N}^{\mathrm{o}}$ termos Aprox. & $\mathrm{K}_{\mathrm{APROX}}$ & $\mathrm{E}(\%)$ \\
\hline 1 & 1,4086 & 60,61 \\
\hline 2 & 0,8571 & 2,25 \\
\hline 3 & 0,883 & 0,68 \\
\hline
\end{tabular}


Com os resultados obtidos mostrados na tabela 4.8 , observa-se que tanto a precisão quanto o tipo de convergência do método mantêm-se nos mesmos padrões dos obtidos para o modo I de abertura. Claramente, o número de termos necessários para obtenção de um resultado com boa precisão (menor do que $1 \%$ de erro) continua sendo governado pela característica do carregamento e tipo de variação destas tensões originadas nas linhas de fissura do subproblema $P_{G}^{(0)}$.

\subsubsection{Exemplo 2: Chapa com Duas Fissuras - Carregamento Uniformemente Distribuído}

No exemplo deste item o carregamento cisalhante aplicado é constante e unitário, porém inclui-se uma fissura a mais para que o método possa capturar a interação entre as fissuras. Os dados referentes às propriedades do material, tipo de malhas e condições de contorno são os mesmos adotados no exemplo anterior.

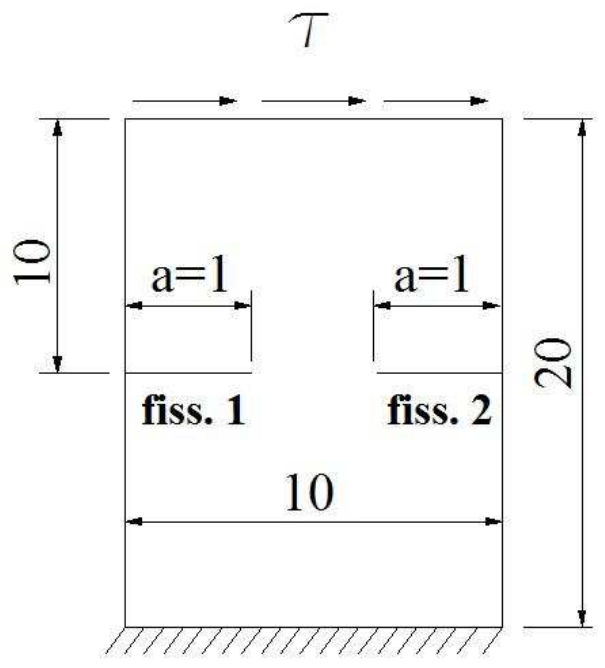

Figura 4.8 - Chapa com 2 fissuras e carregamento constante unitário cisalhante

Os valores-referência de $\mathrm{K}_{\mathrm{II}}$ obtidos mediante análise numérica através do programa ANSYS para as duas fissuras são dados na tabela 4.8:

Tabela 4.8 - Valores-Referência de $K_{I I}$ para carregamento constante

\begin{tabular}{|c|c|}
\hline Fissura & $\mathbf{K}_{\mathbf{I I}}$ \\
\hline 1 & 0,67 \\
\hline 2 & 0,67 \\
\hline
\end{tabular}


Os resultados obtidos com o Método da Partição na determinação dos fatores de intensidade de tensão para cada uma das fissuras estão descritos na tabela 4.9:

Tabela 4.9 - Valores de $K_{\mathrm{II}}$ aproximados obtidos para carregamento constante ( 2 fissuras)

\begin{tabular}{|c|c|c|c|c|c|}
\hline \multicolumn{5}{|c|}{ Carregamento Constante } \\
\hline \multicolumn{3}{|c|}{ Fissura 1: K } & \multicolumn{3}{c|}{ Fissura 2: $\mathbf{K}_{\text {REF }}=\mathbf{0 , 6 7}$} \\
\hline $\begin{array}{c}\mathbf{N}^{\mathbf{0}} \text { termos } \\
\text { Aprox. }\end{array}$ & KAPROx $_{\text {APro }}$ & $\mathbf{E}(\%)$ & $\begin{array}{c}\mathbf{N}^{\mathbf{0}} \text { termos } \\
\text { Aprox. }\end{array}$ & K $_{\text {APROx }}$ & E(\%) \\
\hline 1 & 0,5446 & 18,7 & 1 & 0,5446 & 18,7 \\
\hline 2 & 0,6549 & 2,25 & 2 & 0,6489 & 3,14 \\
\hline 3 & 0,6686 & 0,20 & 3 & 0,6669 & 0,47 \\
\hline
\end{tabular}

Um fato interessante a notar neste exemplo é que mesmo o carregamento na face superior da chapa sendo constante, foram necessários três termos para construir a aproximação na linha da fissura. Tal fato ocorre devido a este carregamento proporcionar uma variação (não-constante) das tensões de cisalhamento ao longo das linhas de fissura em $P_{G}^{(0)}$ (contidas no eixo x, ver figura 4.9). Sendo assim, para se obter resultados mais precisos foram necessários além do termo constante, os termos linear e quadrático.

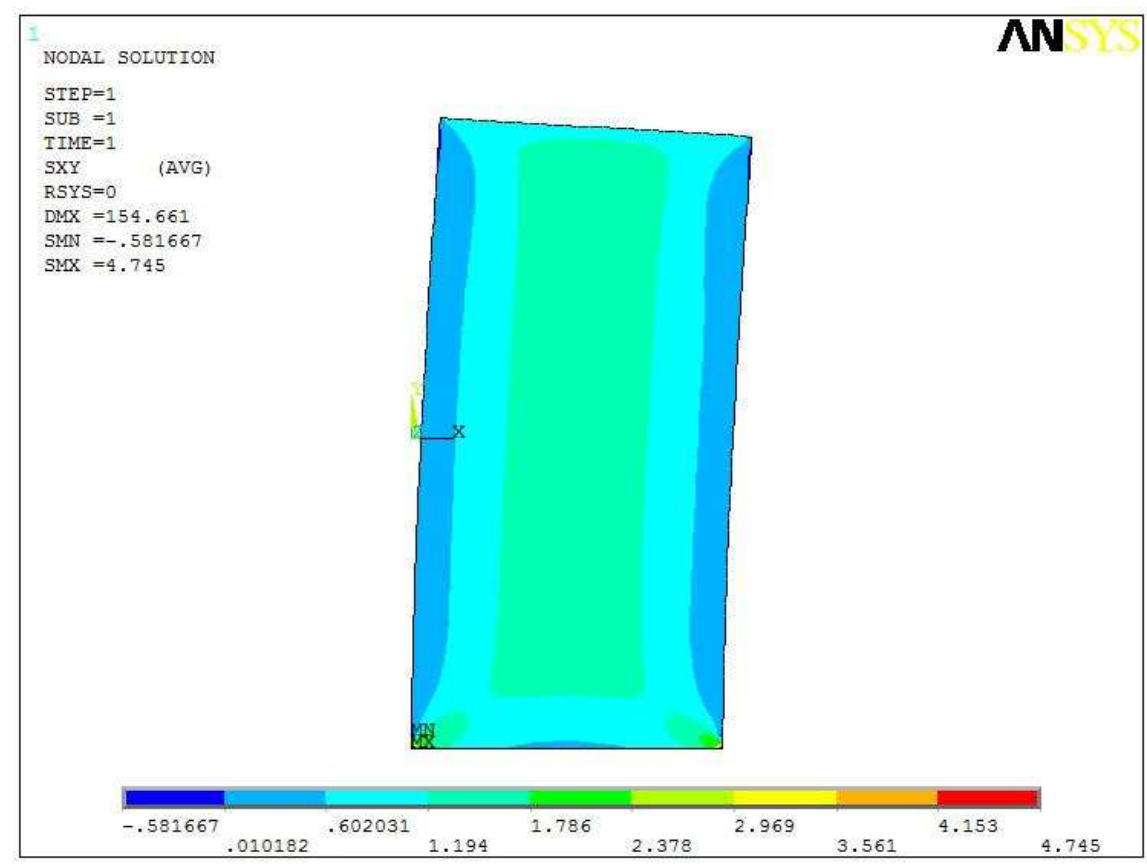

Figura 4.9 - Variação das tensões de cisalhamento nas linhas de fissura (contidas no eixo x) de $P_{G}(0)$ 


\subsubsection{Exemplo 3: Chapa com Duas Fissuras - Carregamento Não-Uniforme}

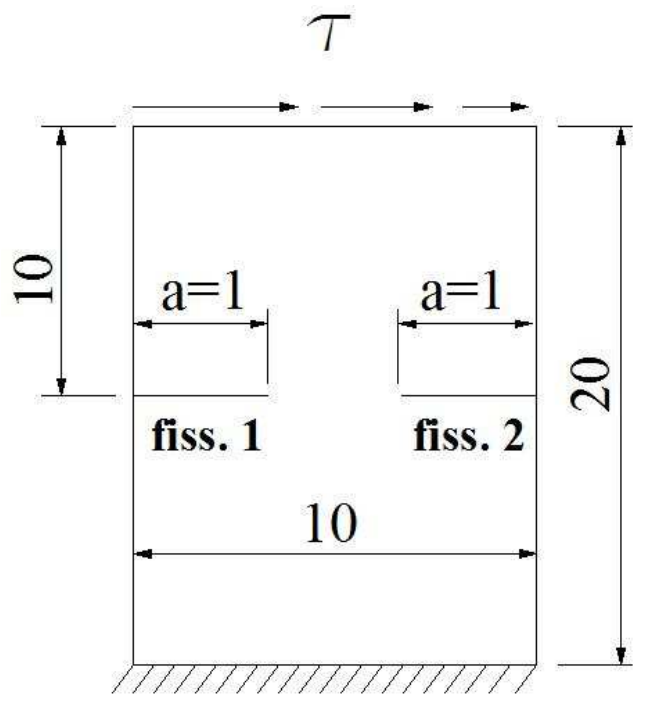

Figura 4.10 - Chapa com 2 fissuras e carregamento cte+quadrático cisalhante

Este exemplo é similar ao do item anterior, porém com o carregamento aplicado com variação constante+quadrático. Observa-se nos resultados apresentados na tabela 4.10 que também são necessários três termos de aproximação para a obtenção de resultados com boa precisão. Isto se dá devido à variação do cisalhamento nas linhas de fissura de $P_{G}^{(0)}$ não serem tão diferentes daquelas ocasionadas pelo carregamento cisalhante constante do exemplo anterior.

Tabela 4.10 - Valores de $K_{\mathrm{II}}$ aproximados obtidos para carregamento Cte+quadrático (2 fissuras)

\begin{tabular}{|c|c|c|c|c|c|}
\hline \multicolumn{5}{|c|}{ Carregamento Constante } \\
\hline \multicolumn{3}{|c|}{ Fissura 1: K } & \multicolumn{3}{c|}{ Fissura 2: $\mathbf{K}_{\text {REF }}=\mathbf{0 , 9 0 4}$} \\
\hline $\begin{array}{c}\mathbf{N}^{\mathbf{0}} \text { termos } \\
\text { Aprox. }\end{array}$ & $\mathbf{K}_{\text {APROx }}$ & $\mathbf{E}(\%)$ & $\begin{array}{c}\mathbf{N}^{\mathbf{0}} \text { termos } \\
\text { Aprox. }\end{array}$ & $\mathbf{K}_{\text {APROx }}$ & E(\%) \\
\hline 1 & 1,4035 & 59,13 & 1 & 1,4389 & 59,17 \\
\hline 2 & 0,8622 & 2,24 & 2 & 0,8758 & 3,12 \\
\hline 3 & 0,8802 & 0,21 & 3 & 0,9002 & 0,42 \\
\hline
\end{tabular}




\subsection{Resolução dos Casos do Método da Partição Utilizando o Programa ANSYS e a Linguagem de Programação Fortran}

A alternativa encontrada para a implementação computacional do Método da Partição baseia-se na utilização do programa ANSYS, juntamente com programas auxiliares compilados através do FORTRAN. Em resumo, busca-se no programa ANSYS a resolução numérica de todos os subproblemas $\left(P_{G}^{(0)}, P_{L}^{(k)}\right.$ e $\left.P_{G}^{(k)}\right)$, sendo que através dos programas auxiliares gerados pelo FORTRAN torna-se possível a geração dos diferentes tipos de carregamentos aproximativos aplicados nas faces de fissuras, além da aplicação do vetor de deslocamento no contorno ${ }^{(i)} \Gamma$ (obtidos nos subproblemas locais) em seus respectivos subproblemas $P_{G}^{(k)}$. Em uma etapa final todos os valores de tensão nas linhas de fissuras e demais dados complementares (tais como coordenadas das fissuras, valores-referência dos fatores de intensidade de tensão, etc) são inseridos em um programa compilado pelo FORTRAN para a resolução do sistema linear $[\mathrm{IG}]\{\alpha\}=\{r\}$, obtendo-se, desta forma, os fatores de escala $\alpha_{i}$ que possibilitarão a obtenção da resposta do problema em termos de fatores de intensidade de tensão.

A seguir são mencionadas estas etapas com maiores detalhes para a resolução do Método da Partição:

a) ANSYS - Modelagem dos subproblemas:

Adota-se as malhas para resolução dos subproblemas, sendo que na maioria dos casos utilizou-se elemento quadrilateral de oito nós, com dois graus de liberdade por nó (deslocamentos em duas direções ortogonais entre si), incluindo o contorno arbitrário. Propriedades do material, condições de contorno (deslocamentos e forças) são definidas ao sistema.

b) ANSYS - Resolução do subproblema global $P_{G}^{(0)}$ :

Após a sua resolução coletam-se os valores de tensão nas linhas de fissura. 
c) FORTRAN - Definição das forças aplicadas nas faces de fissura de $P_{L}^{(k)}$ :

Com um primeiro programa auxiliar implementado via FORTRAN, busca-se inserir neste as coordenadas dos nós referentes à cada face de fissura e também o referido termo de aproximação a ser aplicado. Desta maneira o programa gerará um arquivo *.txt contendo os valores de cargas nodais equivalentes para o dado termo de aproximação. Estes valores estão formatados em linguagem LOGFILE (ver figura 4.11) de tal maneira a serem inseridos no campo "ANSYS Command Prompt" (ver figura 4.12) do programa ANSYS.

$$
\begin{aligned}
& \text { /PREP7 } \\
& \text { FLST,2,1,1,ORDE, } 1 \\
& \text { FITEM,2, Número do nó } \\
& \text { !* } \\
& \text { /GO } \\
& \text { F , P51X , FX , Valor da força } \\
& \text { obs.: FX indica força aplicada na } \\
& \text { direção X }
\end{aligned}
$$

Figura 4.11 - Exemplo de formatação de força nodal aplicada em linguagem LOGFILE

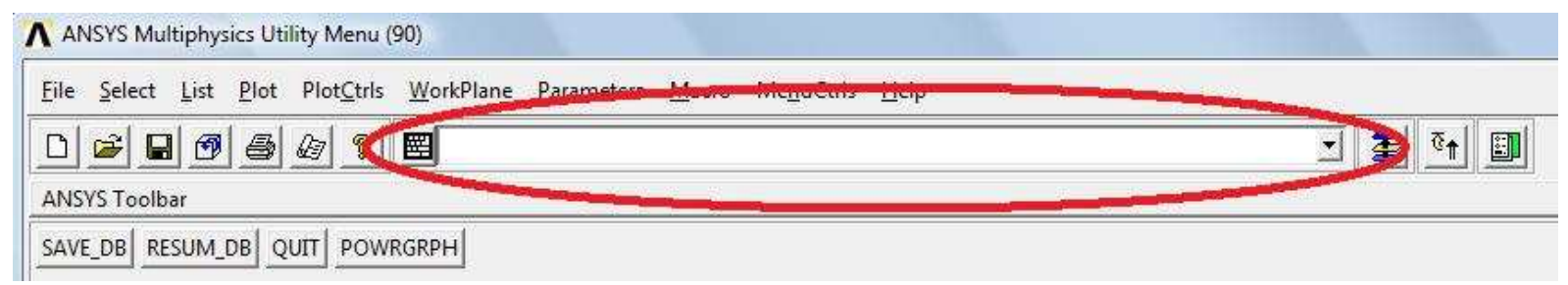

Figura 4.12 - Campo "ANSYS Command Prompt"

d) ANSYS - Resolução do subproblema local $P_{L}^{(k)}$ :

Uma vez definido o carregamento aplicado na face de fissura, resolve-se através do ANSYS este subproblema coletando-se os valores de deslocamentos nos nós pertencentes à face da fissura, assim como os valores de deslocamento no contorno ${ }^{(i)} \Gamma$. Utilizam-se os valores de 
deslocamentos nodais na face de fissura para a obtenção do fator de intensidade local (ver item 3.6.2).

e) FORTRAN - Definição dos deslocamentos a serem aplicados no contorno ${ }^{(i)} \Gamma$ de $P_{G}^{(k)}$ :

Com um segundo programa auxiliar implementado via FORTRAN, busca-se inserir neste os números dos nós do contorno ${ }^{(i)} \Gamma$ assim como os valores dos deslocamentos nodais obtidos (neste mesmo contorno) em $P_{L}^{(k)}$. Sendo assim, este segundo programa gerará um arquivo *.txt contendo os valores de deslocamentos nodais a serem impostos no contorno ${ }^{(i)} \Gamma$ do subproblema $P_{G}^{(k)}$. Estes valores estão formatados em linguagem LOGFILE (ver figura 4.13) de tal maneira a serem inseridos no campo "ANSYS Command Prompt" (ver figura 4.12) do programa ANSYS. Note que a numeração dos nos dos contornos ${ }^{(i)} \Gamma$ tanto de $P_{L}^{(k)}$ quanto de $P_{G}^{(k)}$ têm de serem iguais.

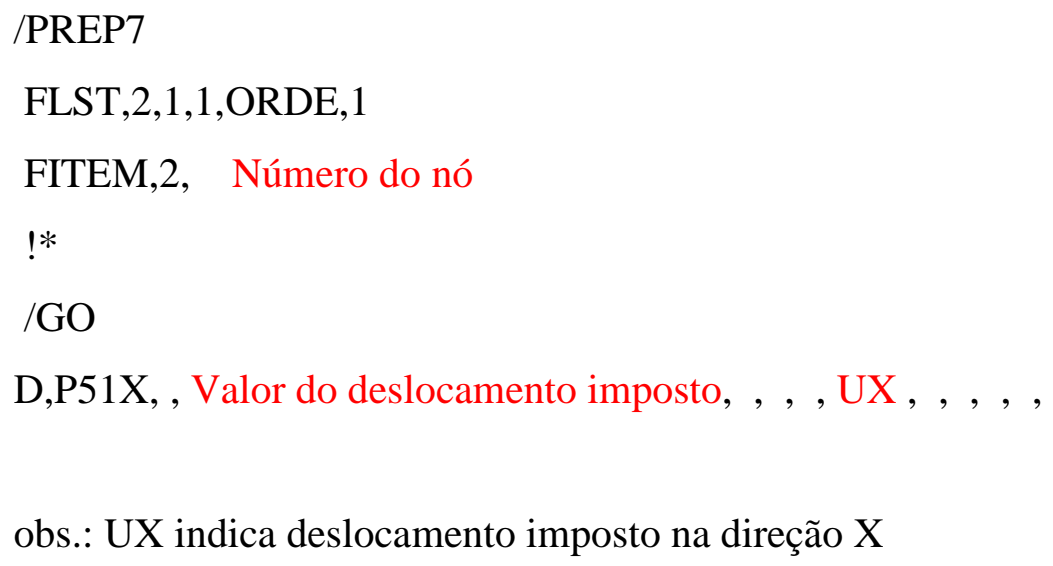

Figura 4.13 - Exemplo de formatação de deslocamento nodal imposto em linguagem LOGFILE

f) ANSYS - Resolução do subproblema local $P_{G}^{(k)}$ :

Uma vez definidos os deslocamentos a serem impostos no contorno ${ }^{(i)} \Gamma$, resolve-se através do ANSYS este subproblema coletando-se os valores de tensões nas linhas de fissuras de interesse. 
g) FORTRAN: Resolução do sistema linear $[\mathrm{IG}]\{\alpha\}=\{r\}$

Nesta última etapa os valores de tensão nas linhas de fissuras e demais dados complementares (tais como coordenadas das fissuras, valores-referência dos fatores de intensidade de tensão, etc) são inseridos em um programa elaborado pelo FORTRAN. Neste programa resolve-se o sistema linear $[\mathrm{IG}]\{\alpha\}=\{r\}$, obtendo-se, desta forma, os fatores de escala $\alpha_{i}$ que propiciarão a resposta do problema em termos de fatores de intensidade de tensão. 


\section{Combinação de Fissuras Internas, de Borda e Fissura-Furo em Modo I de Abertura}

Este capítulo trata da extensão do Método da Partição para a consideração de fissuras internas em sua formulação, uma vez que a formulação original proposta por Andersson, Babuška e Stehlin (1998) é restrita aos casos contendo fissuras de borda. Em uma primeira etapa apresenta-se a formulação para sólidos contendo somente fissuras internas, seguido de exemplos contemplando o modo I de abertura. Evidentemente a formulação pode ser estendida para o modo II de abertura. Em uma segunda etapa apresentam-se casos de fissuras com origem nas bordas de um furo, sendo o destaque principal de estudo o tipo de base aproximativa do campo de tensão na linha da fissura a ser utilizada nestes casos. Finalmente, em uma terceira e última etapa deste capítulo apresentam-se exemplos do emprego do Método da Partição para a resolução de problemas contendo diferentes tipos de fissuras bem como uma metodologia auxiliar para a montagem do sistema linear [IG]. $\{\alpha\}=\{\mathrm{r}\}$. 


\subsection{A Formulação Estendida do Método para Casos que Contemplam Fissuras Internas}

Tendo como base a extensão dos princípios utilizados anteriormente na determinação do Método da Partição para um caso onde duas fissuras internas são consideradas, o problema original $P_{G}$ pode ser redefinido como sendo uma combinação de subproblemas conforme ilustrado na figura 5.1 :

$$
P_{G} \quad P_{G}^{(0)} \quad \sum_{k=1}^{M} \alpha_{k} \cdot P_{L}^{(k)} \quad \sum_{k=1}^{M} \alpha_{k} \cdot P_{G}^{(k)}
$$

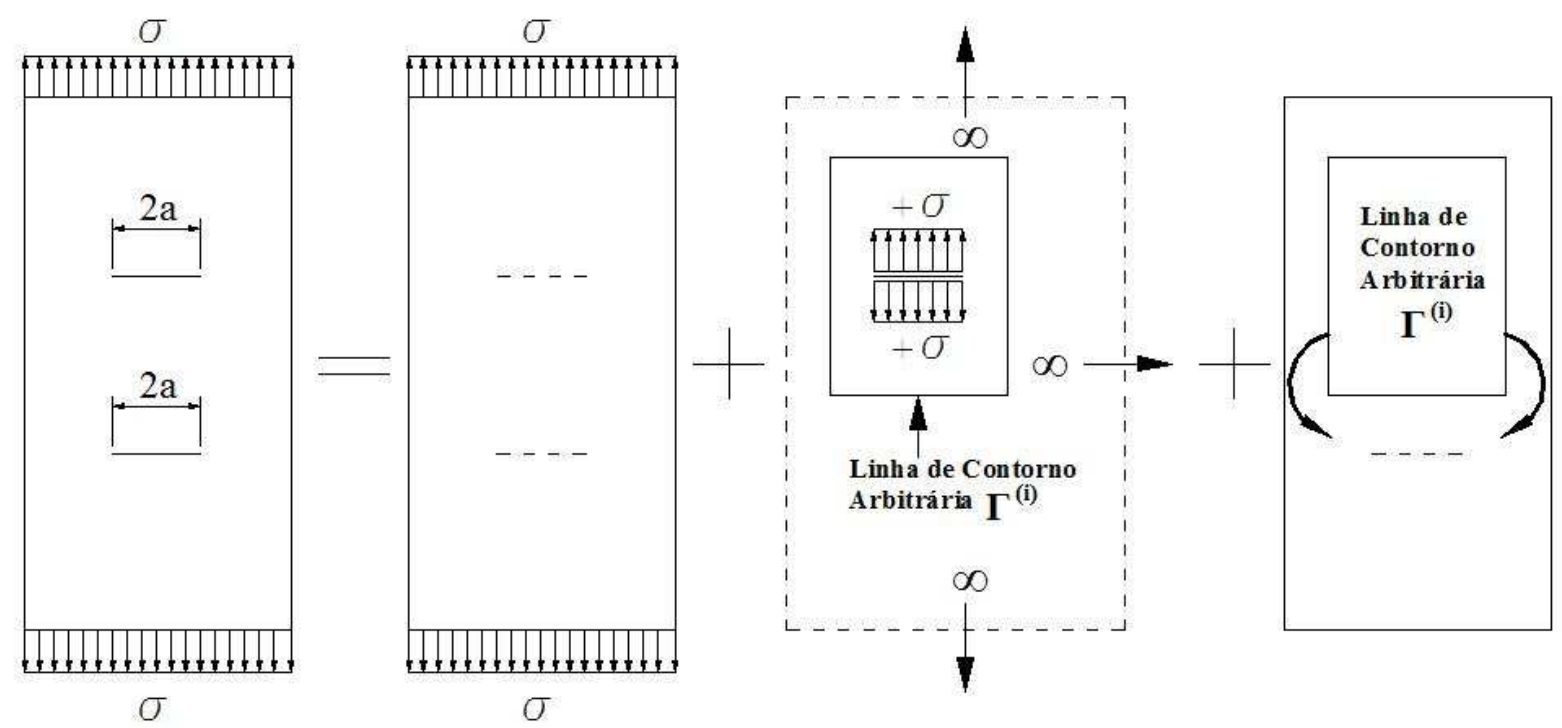

Figura 5.1 - Método da Partição para duas fissuras internas

Lembre-se que o limite dos somatórios indicados é definido por:

I - número de fissuras

J - número de termos da aproximação

$\mathrm{M}=\mathrm{I} \times \mathrm{J}$

Uma vez caracterizados os subproblemas segundo o Método da Partição ressaltam-se algumas mudanças na formulação em virtude da presença da fissura interna. Sendo a formulação anterior baseada no tratamento de fissuras de borda, neste caso havia somente 
uma ponta de fissura para a qual se extraía o fator de intensidade de tensão. Com a consideração das fissuras internas, para cada fissura passam a ser dois os valores de fatores de intensidade de tensão a determinar. Sendo assim, associa-se a cada ponta de fissura um eixo de coordenadas local abrangendo o seu respectivo semi-comprimento conforme ilustra a figura 5.2:

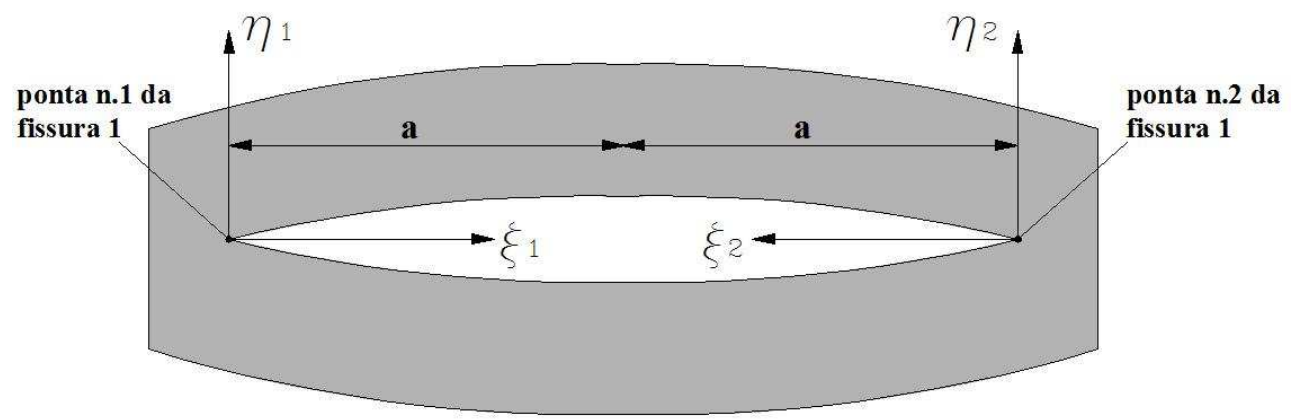

Figura 5.2 - Sistemas de Coordenadas Locais para fissuras internas.

O importante nesta definição do sistema local é o destaque dado a cada um dos dois semi-comprimentos da fissura, particularmente para fins de determinação dos fatores de intensidade de tensão dos subproblemas locais de $P_{L}^{(k)}$. Assim sendo, os pontos contidos no semi-comprimento " $a$ " do eixo local $\xi_{1}$ contribuirão para a determinação do fator de intensidade de tensão da ponta de número 1 desta fissura enquanto os pontos contidos no semi-comprimento " $\boldsymbol{a}$ " do eixo local $\xi_{2}$ contribuirão para a determinação do fator de intensidade de tensão da ponta de número 2 desta mesma fissura interna. Por conseqüência, se em uma análise de casos desta natureza opta-se pela utilização de apenas um termo da aproximação, então deverão ser coletados um valor de tensão para cada ponta de fissura.

O subproblema $P_{G}^{(0)}$ considera o domínio do problema original com as condições de contorno prescritas em deslocamentos e forças aplicadas, porém sem a consideração de fissuras. Neste subproblema, a meta da análise refere-se à coleta dos valores de tensão junto às linhas de fissuras. Sendo assim, coletam-se valores de tensão em pontos nas linhas de fissuras e constrói-se uma aproximação para sua distribuição mediante uma combinação linear de uma base de funções aproximadoras. No caso, têm-se duas relações representativas do campo de tensão aproximado. A primeira refere-se a todas as primeiras pontas de fissura de 
cada fissura interna $\boldsymbol{i}$, e a segunda equação refere-se a todas as segundas pontas de fissura de cada fissura interna $\boldsymbol{i}$ do subproblema em questão. A relação mencionada escreve-se como:

$$
\underline{t}_{G}^{(0)} \approx\left\{\begin{array}{lcc}
\sum_{j=1}^{J} b_{2 i-1, j} \cdot Q_{j}\left(\xi_{1 \text { as pontas }} / a_{i}\right) & p / & 0 \leq \xi_{1 \text { as pontas }} \leq a_{i} \\
\sum_{j=1}^{J} b_{2 i, j} \cdot Q_{j}\left(\xi_{2 \text { as pontas }} / a_{i}\right) & p / & 0 \leq \xi_{\text {2as pontas }} \leq a_{i}
\end{array}\right.
$$

O subproblema local $P_{L}^{(k)}$ consiste em uma fissura interna contida em um domínio $\Gamma^{(i)}$ pré-definido. A diferença básica em relação à formulação das fissuras de borda refere-se à necessidade de extração de dois fatores de intensidade de tensão que podem ou não ser iguais, a depender das vizinhanças deste contorno $\Gamma^{(i)}$. Como ilustra a figura 5.3, no primeiro caso, por conta da simetria, fica evidente que os fatores de intensidade de tensão são iguais, o que não ocorre com o segundo caso onde as vizinhanças do contorno $\Gamma^{(i)}$ afetam diferentemente as duas pontas de fissura.
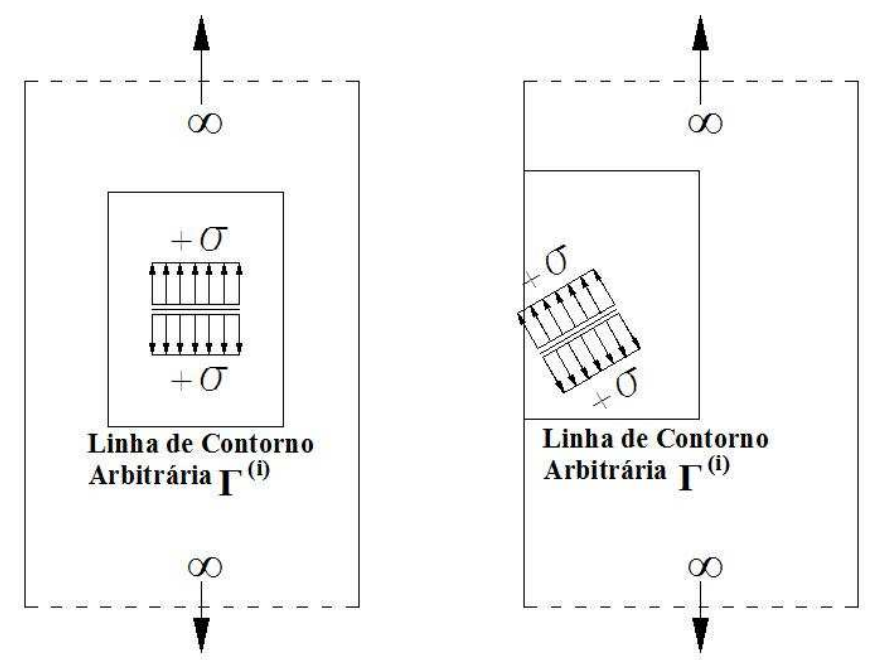

Figura 5.3 - Influências da vizinhança do contorno $\Gamma^{(i)}$ na determinação dos fatores de intensidade de tensão

Outro aspecto importante a ressaltar no subproblema local $P_{L}^{(k)}$ refere-se à aplicação nas faces de fissura dos carregamentos representados pelas componentes $Q_{j}$, da base de aproximação adotada. Como mencionado anteriormente, cada ponta de fissura deve ser tratada de modo distinto, ou seja, o carregamento $Q_{j}$ aplicado no primeiro semi-comprimento 
da fissura interna contribui para o valor do fator de intensidade de tensão da ponta de fissura número 1 , enquanto que o carregamento $Q_{j}$ aplicado no segundo semi-comprimento da fissura interna contribui para o valor do fator de intensidade de tensão da ponta de fissura número 2. Porém, em todos os subproblemas locais, o carregamento $Q_{j}$ é aplicado em ambos os semi-comprimentos da fissura ao mesmo tempo sendo obtidos posteriormente os dois valores dos fatores de intensidade de tensão locais assim como os valores de deslocamentos no contorno $\Gamma^{(i)}$ que serão úteis para a determinação da influência desta fissura interna nas demais linhas de fissuras. Na figura 5.4 apresentam-se a distribuição destes carregamentos $Q_{j}$ para os três primeiros termos de aproximação:

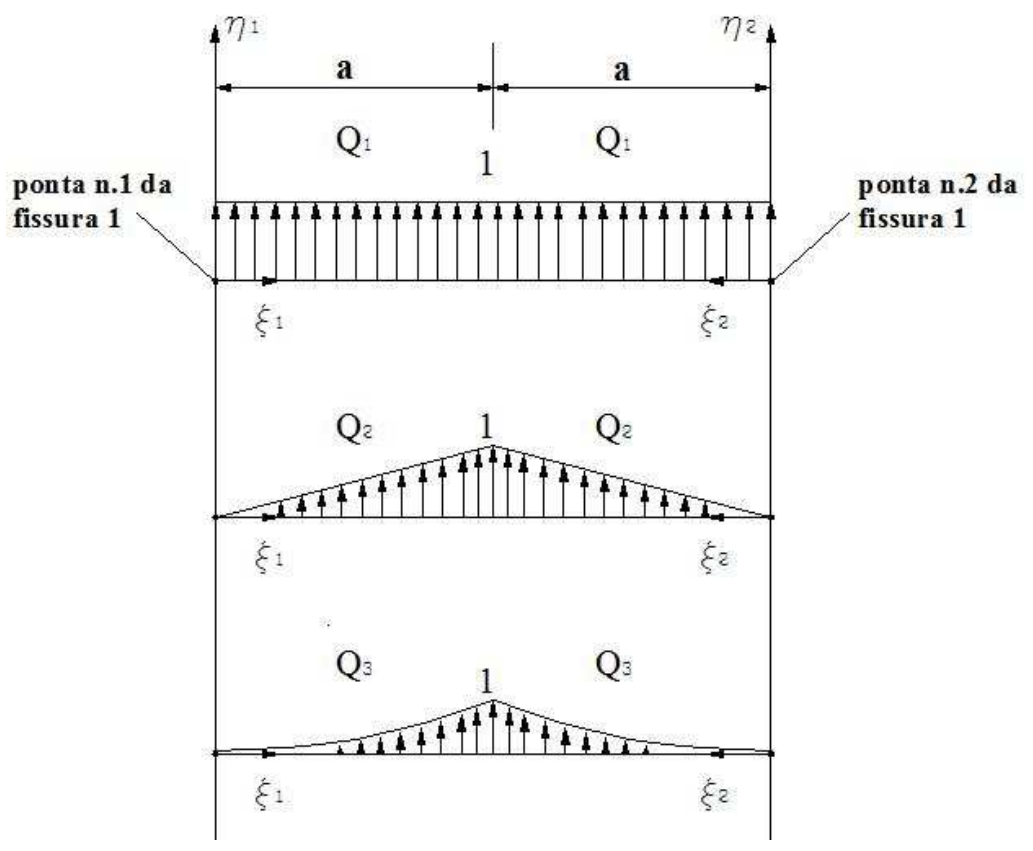

Figura 5.4 - Carregamentos aproximativos Q para fissuras internas

Sendo assim, o campo de tensões para cada fissura interna $i$ pode ser representado da seguinte forma:

$$
\underline{t}_{L}^{(k)}=\left\{\begin{array}{lll}
-\sum_{j=1}^{J} \alpha_{j+(i-1) 2 J} \cdot Q_{j}\left(\xi_{1 \text { as pontas }} / a_{i}\right) & p / & 0 \leq \xi_{1 \text { as pontas }} \leq a_{i} \\
-\sum_{j=1}^{J} \alpha_{j+(2 i-1) J} \cdot Q_{j}\left(\xi_{\text {2as pontas }} / a_{i}\right) & p / & 0 \leq \xi_{\text {2as pontas }} \leq a_{i}
\end{array}\right.
$$


Os fatores de intensidade de tensão ficam definidos da seguinte forma:

$$
\begin{array}{ll}
K^{(2 i-1)}=\sum_{j=1}^{J} \alpha_{j+(i-1) 2 J} \cdot K_{j}^{(2 i-1)} & \text { (primeira ponta da fissura interna } i) \\
K^{(2 i)}=\sum_{j=1}^{J} \alpha_{j+(2 i-1) J} \cdot K_{j}^{(2 i)} & \text { (segunda ponta da fissura interna } i)
\end{array}
$$

Por último, destaca-se o subproblema $P_{G}^{(k)}$, responsável na formulação por inserir a interação entre as fissuras. Neste procedimento, lembra-se que um contorno $\Gamma^{(i)}$ que envolve uma determinada linha de fissura interna, e ao qual estão atrelados valores de deslocamentos, influenciará todas as demais linhas de fissuras localizadas na parte externa a ele. No caso, cada semi-comprimento de uma dada linha de fissura interna $\boldsymbol{i}$ influenciada deverá contribuir com no mínimo um ponto para a construção da aproximação do campo de tensões. Sendo que cada ponto deste (valor de tensão) contribuirá para a determinação do fator de intensidade de tensão da ponta de fissura pertencente ao semi-comprimento ao qual este ponto pertence. Uma vez coletados os valores de tensões nas linhas de fissura, estes são reescritos como uma combinação linear das funções aproximadoras:

$$
\underline{t}_{G}^{(k)}=\left\{\begin{array}{lll}
\sum_{j=1}^{J} \sum_{k=1}^{M} \alpha_{k} \cdot c_{2 i-1, j}^{(k)} \cdot Q_{j}\left(\xi_{1 \text { as pontas }} / a_{i}\right) & p / & 0 \leq \xi_{1 \text { as pontas }} \leq a_{i} \\
\sum_{j=1}^{J} \sum_{k=1}^{M} \alpha_{k} \cdot c_{2 i, j}^{(k)} \cdot Q_{j}\left(\xi_{\text {2as pontas }} / a_{i}\right) & p / & 0 \leq \xi_{\text {2as pontas }} \leq a_{i}
\end{array}\right.
$$

\subsubsection{A Solução Numérica para Casos Envolvendo Fissuras Internas}

Analogamente à formulação para fissuras de borda, a solução para dado problema $P_{G}$ é tal que nas faces de cada uma das fissuras internas de comprimento $2 a_{i}$ o vetor de tensão $\underline{t}$ deve ser nulo. Em forma ponderada ou fraca esta condição pode, para a fissura $i$ de comprimento $2 a_{i}$, ser escrita da seguinte forma: 
$\int_{0}^{2 a_{i}} \underline{t}^{T} \underline{Q}_{j 2}\left(\xi_{i} / a_{i}\right) d \xi=0 \quad c / j 2=1, \ldots, J$

Devido à consideração da independência de cada semi-comprimento $\boldsymbol{a}_{\boldsymbol{i}}$ de cada fissura interna no que diz respeito à obtenção dos fatores de intensidade de tensão, a equação (5.5) pode ser desmembrada em duas, sendo a primeira representativa das tensões nas linhas de fissura situadas no primeiro semi-comprimento de cada fissura interna, e a segunda equação referente às tensões nas linhas de fissura situadas no segundo semi-comprimento de cada fissura interna:

$$
\begin{aligned}
& \int_{0}^{a_{i}} t^{T} Q_{j 2}\left(\xi_{1 \text { las pontas }} / a_{i}\right) d \xi=0 \text { c/ } j 2=1, \ldots, J \\
& \int_{0}^{a_{i}} t^{T} Q_{j 2}\left(\xi_{\text {2as pontas }} / a_{i}\right) d \xi=0 \quad c / j 2=1, \ldots, J
\end{aligned}
$$

O vetor $\boldsymbol{t}$ representa a soma das tensões de todos os subproblemas em cada linha de fissura:

$\underline{t}=\underline{t}_{G}^{(0)}+\underline{t}_{L}^{(k)}+\underline{t}_{G}^{(k)}$

e conforme o item anterior, as tensões são dadas para cada uma das equações definidas em (5.6):

$\underline{t}_{G}^{(0)} \approx\left\{\begin{array}{lcc}\sum_{j=1}^{J} b_{2 i-1, j} \cdot Q_{j}\left(\xi_{1 \text { as pontas }} / a_{i}\right) & p / & 0 \leq \xi_{1 \text { as pontas }} \leq a_{i} \\ \sum_{j=1}^{J} b_{2 i, j} \cdot Q_{j}\left(\xi_{2 a \text { pontas }} / a_{i}\right) & p / & 0 \leq \xi_{\text {2as pontas }} \leq a_{i}\end{array}\right.$

$\underline{t}_{L}^{(k)}=\left\{\begin{array}{lll}-\sum_{j=1}^{J} \alpha_{j+(i-1) 2 J} \cdot Q_{j}\left(\xi_{1 \text { as pontas }} / a_{i}\right) & p / & 0 \leq \xi_{1 \text { as pontas }} \leq a_{i} \\ -\sum_{j=1}^{J} \alpha_{j+(2 i-1) J} \cdot Q_{j}\left(\xi_{\text {2as pontas }} / a_{i}\right) & p / & 0 \leq \xi_{\text {2as pontas }} \leq a_{i}\end{array}\right.$ 


$$
\underline{t}_{G}^{(k)}=\left\{\begin{array}{lll}
\sum_{j=1}^{J} \sum_{k=1}^{M} \alpha_{k} \cdot c_{2 i-1, j}^{(k)} \cdot Q_{j}\left(\xi_{\text {las pontas }} / a_{i}\right) & p / & 0 \leq \xi_{\text {1as pontas }} \leq a_{i} \\
\sum_{j=1}^{J} \sum_{k=1}^{M} \alpha_{k} \cdot c_{2 i, j}^{(k)} \cdot Q_{j}\left(\xi_{\text {2as pontas }} / a_{i}\right) & p / & 0 \leq \xi_{\text {2as pontas }} \leq a_{i}
\end{array}\right.
$$

Sendo assim, obedecendo-se os respectivos semi-comprimentos de cada fissura interna, adiciona-se as equações (5.1), (5.2) e (5.3) em (5.7), e posteriormente o resultado em (5.6) resultando em:

$$
\begin{array}{r}
\int_{0}^{a_{i}}\left\{\sum_{j=1}^{J}\left[b_{2 i-1, j}-\alpha_{j+(i-1) 2 J}+\sum_{k=1}^{M} \alpha_{k} \cdot c_{2 i-1, j}^{(k)}\right] Q_{j}\left(\xi_{1 \text { as pontas }} / a_{i}\right) \cdot Q_{j 2}\left(\xi_{1 \text { as pontas }} / a_{i}\right)\right\} d \xi=0 \\
c / j, j 2=1, \ldots, J \\
\int_{0}^{a_{i}}\left\{\sum_{j=1}^{J}\left[b_{2 i, j}-\alpha_{j+(2 i-1) J}+\sum_{k=1}^{M} \alpha_{k} \cdot c_{2 i, j}^{(k)}\right] Q_{j}\left(\xi_{\text {2as pontas }} / a_{i}\right) \cdot Q_{j 2}\left(\xi_{2 \text { as pontas }} / a_{i}\right)\right\} d \xi=0 \\
c / j, j 2=1, \ldots, J
\end{array}
$$

Isolando-se o termo $\alpha$ das duas equações (5.8) e somando-os chega-se ao sistema linear:

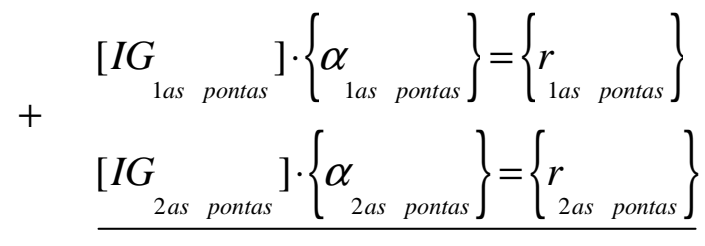

$$
\begin{aligned}
& {[I G] \cdot\{\alpha\}=\{r\}}
\end{aligned}
$$

onde $[I G]$ é a matriz de "influência geral", responsável por manter os termos que definem a interação entre as fissuras. O vetor solução $\{\alpha\}$ define os fatores de escala $\alpha$ responsáveis pela determinação dos fatores de intensidade de tensão para cada fissura conforme indica a relação seguinte: 


$$
\begin{array}{ll}
K^{(2 i-1)}=\sum_{j=1}^{J} \alpha_{j+(i-1) 2 J} \cdot K_{j}^{(2 i-1)} & \quad \text { (primeira ponta da fissura interna } i) \\
K^{(2 i)}=\sum_{j=1}^{J} \alpha_{j+(2 i-1) J} \cdot K_{j}^{(2 i)} \quad & \text { (segunda ponta da fissura interna } i)
\end{array}
$$

onde $K_{j}^{(2 i-1)}$ e $K_{j}^{(2 i)}$ foram calculados nos subproblemas locais $P_{L}^{(k)}$.

Mais detalhes sobre o sistema mencionado por (5.9), assim como a determinação dos termos $\alpha$ são dados a seguir.

\subsubsection{O Sistema Linear [IG]. $\{\alpha\}=\{r\}$ Para Uma Fissura}

As equações que definem a condição essencial do Método da Partição, expressando a nulidade das tensões ao longo da linha da fissura interna $\boldsymbol{i}$ são expressas por:

$$
\begin{array}{r}
\int_{0}^{a_{i}}\left\{\sum_{j=1}^{J}\left[b_{2 i-1, j}-\alpha_{j+(i-1) 2 J}+\sum_{k=1}^{M} \alpha_{k} \cdot c_{2 i-1, j}^{(k)}\right] Q_{j}\left(\xi_{1 \text { as pontas }} / a_{i}\right) \cdot Q_{j 2}\left(\xi_{1 \text { las pontas }} / a_{i}\right)\right\} d \xi=0 \\
c / j, j 2=1, \ldots, J \\
\int_{0}^{a_{i}}\left\{\sum_{j=1}^{J}\left[b_{2 i, j}-\alpha_{j+(2 i-1) J}+\sum_{k=1}^{M} \alpha_{k} \cdot c_{2 i, j}^{(k)}\right] Q_{j}\left(\xi_{2 \text { as pontas }} / a_{i}\right) \cdot Q_{j 2}\left(\xi_{2 a \text { pontas }} / a_{i}\right)\right\} d \xi=0 \\
c / j, j 2=1, \ldots, J
\end{array}
$$

Considerando-se apenas uma única fissura $i=I=1$ e $m$ termos de aproximação $(J=m)$ tem-se que:

$\mathrm{M}=\mathrm{I} . \mathrm{J}=1 . m=m$ 
Sendo um problema sem interação entre fissuras, os respectivos termos das equações (5.8a) e (5.8b) são anulados:

$$
\begin{aligned}
& \sum_{k=1}^{M} \alpha_{k} \cdot c_{2 i-1, j}^{(k)}=0 \\
& \sum_{k=1}^{M} \alpha_{k} \cdot c_{2 i, j}^{(k)}=0
\end{aligned}
$$

e conseqüentemente a equação (5.8) se reduz a:

$$
\begin{aligned}
& \int_{0}^{a_{1}}\left\{\sum_{j=1}^{m}\left[b_{1, j}-\alpha_{j}\right] Q_{j}\left(\xi_{1 \text { as pontas }} / a_{1}\right) \cdot Q_{j 2}\left(\xi_{1 \text { as pontas }} / a_{1}\right)\right\} d \xi=0 \quad c / j, j 2=1, \ldots, J \\
& \int_{0}^{a_{1}}\left\{\sum_{j=1}^{m}\left[b_{2, j}-\alpha_{j+m}\right] Q_{j}\left(\xi_{\text {2as pontas }} / a_{1}\right) \cdot Q_{j 2}\left(\xi_{\text {2as pontas }} / a_{1}\right)\right\} d \xi=0 \quad c / j, j 2=1, \ldots, J
\end{aligned}
$$

Desenvolvendo a equação acima, obtêm-se o seguinte sistema linear:

$$
\left[\begin{array}{cc}
{\left[Q^{(1)}\right]} & {[0]} \\
{[0]} & {\left[Q^{(2)}\right]}
\end{array}\right]_{2 m \times 2 m} \cdot\left\{\begin{array}{c}
\alpha_{1} \\
\alpha_{2} \\
\vdots \\
\alpha_{2 m}
\end{array}\right\}=\left\{\begin{array}{l}
\left\{r l^{(1)}\right\} \\
\left\{r l^{(2)}\right\}
\end{array}\right\}_{2 m}
$$

Onde:

$\therefore$ [0] são sub-matrizes $m \times m$ nulas.

$\therefore\left[Q^{(1)}\right]$ e $\left[Q^{(2)}\right]$ são sub-matrizes $m \times m$ para as pontas de fissura número 1 e 2 respectivamente. 


$$
\left[Q^{(1)}\right]=\left[Q^{(2)}\right]=-\left[\begin{array}{cccc}
\int_{0}^{a 1} Q_{1} Q_{1} \partial \xi & \int_{0}^{a 1} Q_{2} Q_{1} \partial \xi & \cdots & \int_{0}^{a 1} Q_{m} Q_{1} \partial \xi \\
\int_{0}^{a 1} Q_{1} Q_{2} \partial \xi & \int_{0}^{a 1} Q_{2} Q_{2} \partial \xi & \cdots & \int_{0}^{a 1} Q_{m} Q_{1} \partial \xi \\
\vdots & \vdots & \ddots & \vdots \\
\int_{0}^{a 1} Q_{1} Q_{m} \partial \xi & \int_{0}^{a 1} Q_{1} Q_{m} \partial \xi & \cdots & \int_{0}^{a 1} Q_{m} Q_{m} \partial \xi
\end{array}\right]_{m \times m}
$$

$\therefore\left\{r l^{(1)}\right\}$ e $\left\{r l^{(2)}\right\}$ são sub-vetores de comprimento $m$ para as pontas de fissura número 1 e 2 respectivamente. Estes representam as tensões oriundas de $P_{G}^{(0)}$.

$$
\left\{r l^{(1)}\right\}=-\left\{\begin{array}{c}
\int_{0}^{a 1}\left(b_{1,1} Q_{1} Q_{1}+b_{1,2} Q_{2} Q_{1}+\ldots+b_{1, m} Q_{m} Q_{1}\right) \partial \xi \\
\int_{0}^{a 1}\left(b_{1,1} Q_{1} Q_{2}+b_{1,2} Q_{2} Q_{2}+\ldots+b_{1, m} Q_{m} Q_{2}\right) \partial \xi \\
\vdots \\
\int_{0}^{a 1}\left(b_{1,1} Q_{1} Q_{m}+b_{1,2} Q_{2} Q_{m}+\ldots+b_{1, m} Q_{m} Q_{m}\right) \partial \xi
\end{array}\right\}
$$

$$
\left\{r l^{(2)}\right\}=-\left\{\begin{array}{c}
\int_{0}^{a 1}\left(b_{2,1} Q_{1} Q_{1}+b_{2,2} Q_{2} Q_{1}+\ldots+b_{2, m} Q_{m} Q_{1}\right) \partial \xi \\
\int_{0}^{a 1}\left(b_{2,1} Q_{1} Q_{2}+b_{2,2} Q_{2} Q_{2}+\ldots+b_{2, m} Q_{m} Q_{2}\right) \partial \xi \\
\vdots \\
\int_{0}^{a 1}\left(b_{2,1} Q_{1} Q_{m}+b_{2,2} Q_{2} Q_{m}+\ldots+b_{2, m} Q_{m} Q_{m}\right) \partial \xi
\end{array}\right\}
$$

Os dois fatores de intensidade de tensão da fissura interna, ou seja, $K^{(1)}$ e $K^{(2)}$ são dados da seguinte forma:

$$
K^{(1)}=\sum_{j=1}^{m} \alpha_{j} \cdot K_{j}^{(1)} \quad \text { (primeira ponta da fissura interna) }
$$


$K^{(2)}=\sum_{j=1}^{m} \alpha_{j+m} \cdot K_{j}^{(2)} \quad$ (segunda ponta da fissura interna)

onde $K_{j}^{(1)}$ e $K_{j}^{(2)}$ foram calculados nos subproblemas locais $P_{L}^{(k)}$.

\subsubsection{O Sistema Linear [IG]. $\{\alpha\}=\{r\}$ Para Várias Fissuras}

Neste caso consideram-se então as duas equações do Método da Partição que definem a condição de nulidade das tensões na linha da fissura interna $\boldsymbol{i}$ :

Primeiras pontas de fissura:

$\int_{0}^{a_{i}}\left\{\sum_{j=1}^{J}\left[b_{2 i-1, j}-\alpha_{j+(i-1) 2 J}+\sum_{k=1}^{M} \alpha_{k} \cdot c_{2 i-1, j}^{(k)}\right] Q_{j}\left(\xi_{\text {1as pontas }} / a_{i}\right) \cdot Q_{j 2}\left(\xi_{\text {las pontas }} / a_{i}\right)\right\} d \xi=0 \quad c / j, j 2=1, \ldots, J$

Segundas pontas de fissura:

$\int_{0}^{a_{i}}\left\{\sum_{j=1}^{J}\left[b_{2 i, j}-\alpha_{j+(2 i-1) J}+\sum_{k=1}^{M} \alpha_{k} \cdot c_{2 i, j}^{(k)}\right] Q_{j}\left(\xi_{2 a \text { pontas }} / a_{i}\right) \cdot Q_{j 2}\left(\xi_{2 a s \text { pontas }} / a_{i}\right)\right\} d \xi=0 c / j, j 2=1, \ldots, J$

Para $I$ fissuras internas, são dados dois conjuntos de equações referentes às primeiras e segundas pontas de fissuras:

Primeiras pontas de fissura:

$$
\begin{aligned}
& \int_{0}^{a_{1}}\left\{\sum_{j=1}^{J}\left[b_{1, j}-\alpha_{j}+\sum_{k=1}^{M} \alpha_{k} \cdot c_{1, j}^{(k)}\right] Q_{j}\left(\xi_{1 \text { as pontas }} / a_{1}\right) \cdot Q_{j 2}\left(\xi_{1 \text { las pontas }} / a_{1}\right)\right\} d \xi=0 \quad c / j, j 2=1, \ldots, J \\
& \int_{0}^{a_{2}}\left\{\sum_{j=1}^{J}\left[b_{3, j}-\alpha_{j+2 J}+\sum_{k=1}^{M} \alpha_{k} \cdot c_{3, j}^{(k)}\right] Q_{j}\left(\xi_{\text {las pontas }} / a_{2}\right) \cdot Q_{j 2}\left(\xi_{\text {las pontas }} / a_{2}\right)\right\} d \xi=0 c / j, j 2=1, \ldots, J
\end{aligned}
$$




$$
\int_{0}^{a I}\left\{\sum_{j=1}^{J}\left[b_{2 I-1, j}-\alpha_{j+(I-1) 2 J}+\sum_{k=1}^{M} \alpha_{k} \cdot c_{2 I-1, j}^{(k)}\right] Q_{j}\left(\xi_{1 \text { as pontas }} / a_{I}\right) \cdot Q_{j 2}\left(\xi_{1 \text { as pontas }} / a_{I}\right)\right\} d \xi=0 \quad c / j, j 2=1, \ldots, J
$$

Segundas pontas de fissura:

$$
\begin{aligned}
& \int_{0}^{a 1}\left\{\sum_{j=1}^{J}\left[b_{2, j}-\alpha_{j+J}+\sum_{k=1}^{M} \alpha_{k} \cdot c_{2, j}^{(k)}\right] Q_{j}\left(\xi_{2 \text { as pontas }} / a_{1}\right) \cdot Q_{j 2}\left(\xi_{2 \text { as pontas }} / a_{1}\right)\right\} d \xi=0 c / j, j 2=1, \ldots, J \\
& \int_{0}^{a_{2}}\left\{\sum_{j=1}^{J}\left[b_{4, j}-\alpha_{j+3 J}+\sum_{k=1}^{M} \alpha_{k} \cdot c_{4, j}^{(k)}\right] Q_{j}\left(\xi_{2 a s \text { pontas }} / a_{2}\right) \cdot Q_{j 2}\left(\xi_{2 \text { as pontas }} / a_{2}\right)\right\} d \xi=0 c / j, j 2=1, \ldots, J \\
& \vdots \\
& \int_{0}^{a_{I}}\left\{\sum_{j=1}^{J}\left[b_{2 I, j}-\alpha_{j+(2 I-1) J}+\sum_{k=1}^{M} \alpha_{k} \cdot c_{2 I, j}^{(k)}\right] Q_{j}\left(\xi_{\text {2as pontas }} / a_{I}\right) \cdot Q_{j 2}\left(\xi_{2 \text { as pontas }} / a_{I}\right)\right\} d \xi=0 \quad c / j, j 2=1, \ldots, J
\end{aligned}
$$

Adotando-se os seguintes dados:

$$
\begin{array}{llll}
\checkmark & \mathrm{n}^{\mathrm{o}} \text { de termos da aproximação adotados }=m & \Rightarrow & J=m \\
\checkmark & \mathrm{n}^{\mathrm{o}} \text { de fissuras }=\mathrm{n} & \Rightarrow & I=n \\
\checkmark & M=J . I=m . n
\end{array}
$$

e tomando, por exemplo, a primeira equação de (5.16a) e a primeira equação de (5.16b), observa-se que cada uma destas se desdobra em um novo conjunto de equações, sendo o número de equações deste novo conjunto igual ao número de termos da aproximação $(j 2=$ $1, \ldots, J=1, \ldots, m)$ : 
Primeiras pontas de fissura:

$\int_{0}^{a_{1}}\left\{\sum_{j=1}^{J}\left[b_{1, j}-\alpha_{j}+\sum_{k=1}^{M} \alpha_{k} \cdot c_{1, j}^{(k)}\right] Q_{j}\left(\xi_{1 \text { as pontas }} / a_{1}\right) \cdot Q_{1}\left(\xi_{1 \text { as pontas }} / a_{1}\right)\right\} d \xi=0 \quad c / j=1, \ldots, J$
$\int_{0}^{a_{1}}\left\{\sum_{j=1}^{J}\left[b_{1, j}-\alpha_{j}+\sum_{k=1}^{M} \alpha_{k} \cdot c_{1, j}^{(k)}\right] Q_{j}\left(\xi_{\text {las pontas }} / a_{1}\right) \cdot Q_{2}\left(\xi_{\text {las pontas }} / a_{1}\right)\right\} d \xi=0 \quad c / j=1, \ldots, J$

$\int_{0}^{a_{1}}\left\{\sum_{j=1}^{J}\left[b_{1, j}-\alpha_{j}+\sum_{k=1}^{M} \alpha_{k} \cdot c_{1, j}^{(k)}\right] Q_{j}\left(\xi_{\text {las pontas }} / a_{1}\right) \cdot Q_{m}\left(\xi_{\text {las pontas }} / a_{1}\right)\right\} d \xi=0 \quad c / j=1, \ldots, J$

Segundas pontas de fissura:

$\int_{0}^{a 1}\left\{\sum_{j=1}^{J}\left[b_{2, j}-\alpha_{j+J}+\sum_{k=1}^{M} \alpha_{k} \cdot c_{2, j}^{(k)}\right] Q_{j}\left(\xi_{\text {2as pontas }} / a_{1}\right) \cdot Q_{1}\left(\xi_{\text {2as pontas }} / a_{1}\right)\right\} d \xi=0 \quad c / j=1, \ldots, J$
$\int_{0}^{a 1}\left\{\sum_{j=1}^{J}\left[b_{2, j}-\alpha_{j+J}+\sum_{k=1}^{M} \alpha_{k} \cdot c_{2, j}^{(k)}\right] Q_{j}\left(\xi_{\text {2as pontas }} / a_{1}\right) \cdot Q_{2}\left(\xi_{\text {2as pontas }} / a_{1}\right)\right\} d \xi=0 \quad c / j=1, \ldots, J$
$\vdots$
$\int_{0}^{a 1}\left\{\sum_{j=1}^{J}\left[b_{2, j}-\alpha_{j+J}+\sum_{k=1}^{M} \alpha_{k} \cdot c_{2, j}^{(k)}\right] Q_{j}\left(\xi_{\text {2as pontas }} / a_{1}\right) \cdot Q_{m}\left(\xi_{\text {2as pontas }} / a_{1}\right)\right\} d \xi=0 \quad$ c/ $j=1, \ldots, J$

Com isto, desenvolvendo todas as equações expressas por (5.16), chega-se ao seguinte sistema linear:

$$
\begin{aligned}
& +\left[I G_{\text {las pontas }}\right] \cdot\left\{\alpha_{\text {las pontas }}\right\}=\left\{\begin{array}{l}
r \\
\text { 1as pontas }
\end{array}\right\} \\
& \underline{\left[\begin{array} { l } 
{ [ G _ { \text { 2as pontas } } } \\
{ ] }
\end{array} \left\{\left\{\alpha_{\text {2as pontas }}\right\}=\left\{\begin{array}{l}
r \\
\text { 2as pontas }
\end{array}\right\}\right.\right.}
\end{aligned}
$$

$$
[I G] \cdot\{\alpha\}=\{r\}
$$


onde a matriz $[I G]$ de dimensões $2 . m . n \times 2 . m . n$ pode ser decomposta em duas:

$[I G]=[I G]_{L}+[I G]_{G}$

sendo que $[I G]_{L}$ é uma matriz que possui as integrais representativas das tensões em $P_{L}^{(k)}$ e $[I G]_{G}$ possui as integrais responsáveis pelo efeito de interação entre as fissuras.

A matriz $[I G]_{L}$ possui $2 n \times 2 n$ sub-matrizes e é dada da seguinte forma:

$[I G]_{L}=\left[\begin{array}{cccc}{\left[Q^{(1)}\right]} & {[0]} & \cdots & {[0]} \\ {[0]} & {\left[Q^{(2)}\right]} & \cdots & {[0]} \\ \vdots & \vdots & \ddots & \vdots \\ {[0]} & {[0]} & \cdots & {\left[Q^{(2 n)}\right.}\end{array}\right]$

$\therefore$ [0] são sub-matrizes $m \times m$ nulas

$\therefore\left[Q^{(i)}\right]$ são sub-matrizes $m \times m$ para $2 n$ pontas de fissura $(i=1, \ldots, 2 n)$ :

$$
\left[Q^{(i)}\right]=-\left[\begin{array}{cccc}
\int_{0}^{a i} Q_{1} Q_{1} \partial \xi & \int_{0}^{a i} Q_{2} Q_{1} \partial \xi & \cdots & \int_{0}^{a i} Q_{m} Q_{1} \partial \xi \\
\int_{0}^{a i} Q_{1} Q_{2} \partial \xi & \int_{0}^{a i} Q_{2} Q_{2} \partial \xi & \cdots & \int_{0}^{a i} Q_{m} Q_{1} \partial \xi \\
\vdots & \vdots & \ddots & \vdots \\
\int_{0}^{a i} Q_{1} Q_{m} \partial \xi & \int_{0}^{a i} Q_{1} Q_{m} \partial \xi & \cdots & \int_{0}^{a i} Q_{m} Q_{m} \partial \xi
\end{array}\right]_{m \times m}
$$


Por outro lado, $[I G]_{G}$ que possui $2 n \times 2 n$ sub-matrizes é dada da seguinte forma:

$$
[I G]_{G}=\left[\begin{array}{cccccccc}
{\left[c Q_{1}^{(1)}\right]} & {[0]} & {\left[c Q_{3}^{(1)}\right]} & {[0]} & \ldots & \ldots & {\left[c Q_{2 n-1}^{(1)}\right]} & {[0]} \\
{[0]} & {\left[c Q_{2}^{(1)}\right]} & {[0]} & {\left[c Q_{4}^{(1)}\right]} & \ldots & \ldots & {[0]} & {\left[c Q_{2 n}^{(1)}\right]} \\
{\left[c Q_{1}^{(2)}\right]} & {[0]} & {\left[c Q_{3}^{(2)}\right]} & {[0]} & \ldots & \ldots & {\left[c Q_{2 n-1}^{(2)}\right]} & {[0]} \\
{[0]} & {\left[c Q_{2}^{(2)}\right]} & {[0]} & {\left[c Q_{4}^{(2)}\right]} & \ldots & \ldots & {[0]} & {\left[c Q_{2 n}^{(2)}\right]} \\
\vdots & \vdots & \vdots & \vdots & \ddots & \ldots & {\left[c Q_{2 n-1}^{(3)}\right]} & {[0]} \\
\vdots & \vdots & \vdots & \vdots & \vdots & \ddots & {[0]} & {\left[c Q_{2 n}^{(3)}\right]} \\
{\left[c Q_{1}^{(n)}\right]} & {[0]} & {\left[c Q_{3}^{(n)}\right]} & {[0]} & {\left[c Q_{5}^{(n)}\right]} & {[0]} & {\left[c Q_{2 n-1}^{(n)}\right]} & {[0]} \\
{[0]} & {\left[c Q_{2}^{(n)}\right]} & {[0]} & {\left[c Q_{4}^{(n)}\right]} & {[0]} & {\left[c Q_{6}^{(n)}\right]} & {[0]} & {\left[c Q_{2 n}^{(n)}\right.}
\end{array}\right]
$$

onde $\left[c Q_{i}^{(i)}\right]$ são sub-matrizes $m \times m$ para $2 n$ pontas de fissura $(i=1, \ldots, 2 n)$ e para $m$ termos de aproximação $(j=1, \ldots, m)$. [0] são sub-matrizes $m \times m$ nulas.

Considerando-se as sub-matrizes da primeira linha de $[I G]_{G}$ dizem respeito às influências de todas as fissuras sobre a primeira ponta da fissura interna 1. A segunda linha de $[I G]_{G}$ refere-se às influências de todas as fissuras sobre a segunda ponta da fissura interna 1. A terceira linha de $[I G]_{G}$ refere-se às influências de todas as fissuras sobre a primeira ponta da fissura interna 2 e assim sucessivamente.

Para uma melhor compreensão, de todas estas sub-matrizes que compõem as linhas $1 \mathrm{e}$ 2, $\left[c Q_{1}^{(1)}\right]$ representa a influência dos carregamentos aproximativos $j$ aplicados na fissura interna 1 sobre a primeira ponta desta mesma fissura interna. $\left[c Q_{2}^{(1)}\right]$ representa a influência dos carregamentos aproximativos $j$ aplicados nesta fissura interna 1 sobre a segunda ponta desta mesma fissura. $\left[c Q_{3}^{(1)}\right]$ representa a influência dos carregamentos aproximativos $j$ aplicados na fissura interna 2 sobre a primeira ponta da fissura interna 1. $\left[c Q_{4}^{(1)}\right]$ representa a influência dos carregamentos aproximativos $j$ aplicados na fissura interna 2 sobre a segunda ponta da fissura interna 1 e assim sucessivamente. Desta maneira, a soma de todos os elementos de uma linha da matriz $[I G]_{G}$ formam os $\boldsymbol{k}(=1, \ldots, \mathrm{M})$ subproblemas do Método da Partição. 
Conforme ressaltado durante o desenvolvimento da formulação para fissuras de borda, o objetivo da matriz $[I G]_{G}$ é realizar a interação entre as fissuras. Sendo assim, as submatrizes que compõem a diagonal principal desta matriz devem ser eliminadas, uma vez que não tem sentido algum considerar-se o efeito da fissura sobre ela mesma (isto acarretaria em duplicidade de efeitos uma vez que este efeito já está considerado em $P_{L}^{(k)}$ ). Sendo assim $[I G]_{G}$ é dada da seguinte forma:

$$
[I G]_{G}=\left[\begin{array}{cccccccc}
{[0]} & {[0]} & {\left[c Q_{3}^{(1)}\right]} & {[0]} & \ldots & \ldots & {\left[c Q_{2 n-1}^{(1)}\right]} & {[0]} \\
{[0]} & {[0]} & {[0]} & {\left[c Q_{4}^{(1)}\right]} & \ldots & \ldots & {[0]} & {\left[c Q_{2 n}^{(1)}\right]} \\
{\left[c Q_{1}^{(2)}\right]} & {[0]} & {[0]} & {[0]} & \ldots & \ldots & {\left[c Q_{2 n-1}^{(2)}\right]} & {[0]} \\
{[0]} & {\left[c Q_{2}^{(2)}\right]} & {[0]} & {[0]} & \ldots & \ldots & {[0]} & {\left[c Q_{2 n}^{(2)}\right]} \\
\vdots & \vdots & \vdots & \vdots & \ddots & \ldots & {\left[c Q_{2 n-1}^{(3)}\right]} & {[0]} \\
\vdots & \vdots & \vdots & \vdots & \vdots & \ddots & {[0]} & {\left[c Q_{2 n}^{(3)}\right.} \\
{\left[c Q_{1}^{(n)}\right]} & {[0]} & {\left[c Q_{3}^{(n)}\right]} & {[0]} & {\left[c Q_{5}^{(n)}\right]} & {[0]} & {[0]} & {[0]} \\
{[0]} & {\left[c Q_{2}^{(n)}\right]} & {[0]} & {\left[c Q_{4}^{(n)}\right]} & {[0]} & {\left[c Q_{6}^{(n)}\right]} & {[0]} & {[0]}
\end{array}\right]
$$

Definida então a matriz $[I G]$, resta-se destacar o vetor-solução $\{\alpha\}$ :

$$
\{\alpha\}=\left\{\begin{array}{c}
\alpha_{1} \\
\alpha_{2} \\
\vdots \\
\alpha_{2 m . n}
\end{array}\right\}
$$

assim como o vetor $\{r\}$, responsável pelas integrais das tensões nas linhas de fissuras em $P_{G}^{(0)}$, apresentado da seguinte maneira: 


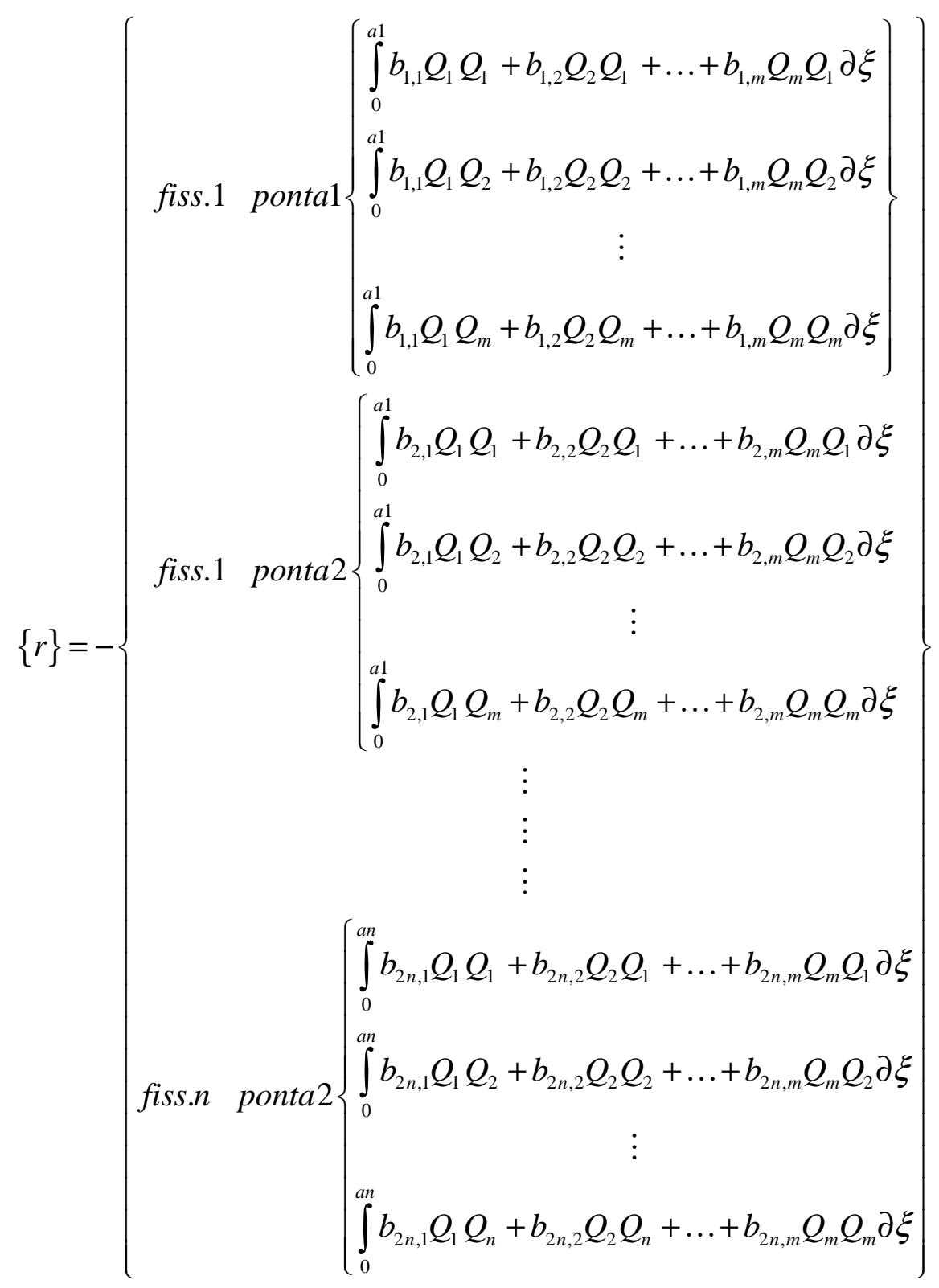

\subsubsection{Exemplo 1: Chapa com Uma Fissura - Carregamento Uniformemente Distribuído}

Neste exemplo considera-se uma chapa com dimensões 10x20 ( $\mathrm{W}=10$ e $\mathrm{H}=20)$, comprimento de fissura $2 \mathrm{a}=2$, e tensão constante unitária $(\sigma=1)$ aplicada ao longo das faces inferior e superior da chapa: 


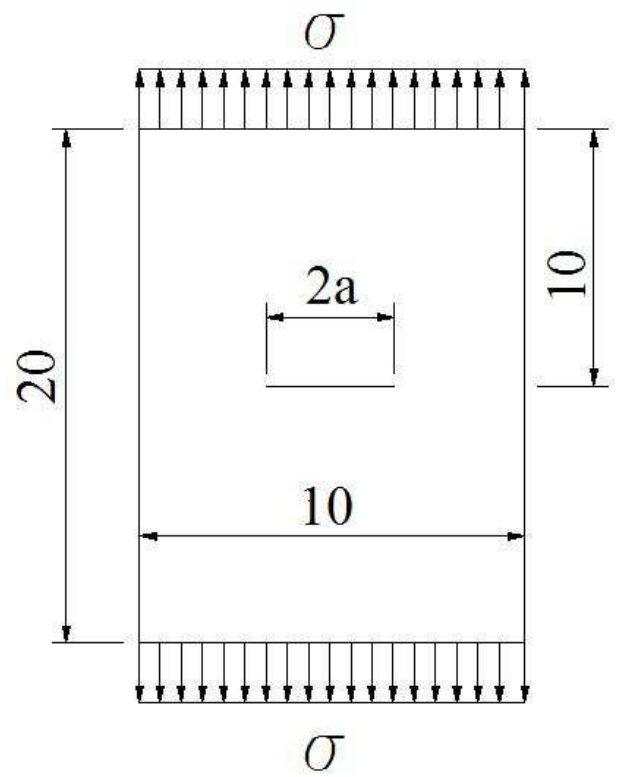

Figura 5.5 - Chapa com 1 fissura e carregamento constante

A solução analítica deste problema pode ser encontrada em Tada, Paris e Irwin (2000) como sendo:

$K_{I}=C \cdot \sigma \cdot \sqrt{\pi a}$

com:

$C=1,0+0,256\left(\frac{a}{W}\right)-1,152\left(\frac{a}{W}\right)^{2}+12,2\left(\frac{a}{W}\right)^{3}$

O fator de intensidade de tensão resulta do seguinte cálculo:

$\frac{a}{W}=\frac{1}{10}=0,1 \Rightarrow \mathrm{C}=1,02628 \Rightarrow K_{I}=C \cdot \sigma \cdot \sqrt{\pi a}=C \cdot 1 \cdot \sqrt{\pi \cdot 1}=1,819034$ 
A resolução deste problema segue a decomposição expressa na figura 5.6:

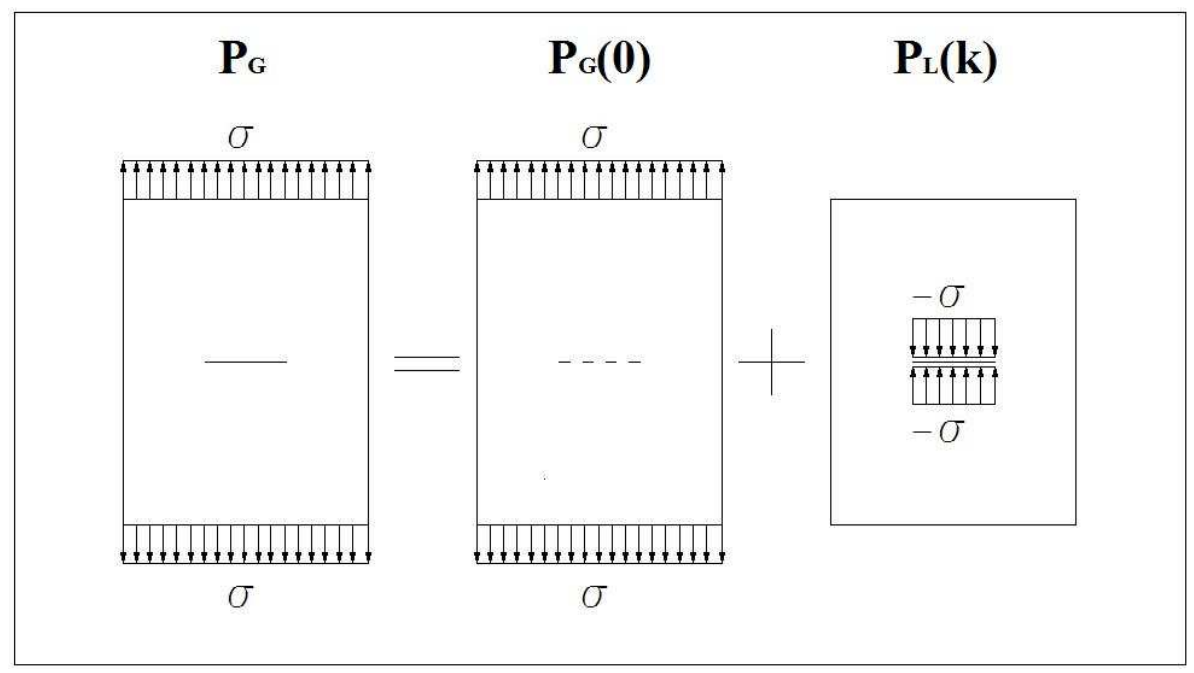

Figura 5.6 - Subdivisão do problema original pelo Método da Partição

Por se tratar de força distribuída uniformemente na chapa, considera-se apenas um termo de aproximação. Assim sendo, os índices para a utilização das equações do Método da Partição ficam definidos por:

$\mathrm{I}=1 \quad\left(\mathrm{n}^{\mathrm{o}}\right.$ de fissuras internas $)$

$\mathrm{J}=1 \quad\left(\mathrm{n}^{\mathrm{o}}\right.$ de termos da aproximação)

$\mathrm{M}=\mathrm{I} . \mathrm{J}=1$

O termo da base de aproximação resulta:

$Q_{j}\left(\xi_{i} / a_{i}\right)=\left(\frac{\xi_{i}}{a_{i}}\right)^{j-1} \quad \Rightarrow \quad Q_{1}\left(\xi_{1} / a_{1}\right)=\left(\frac{\xi_{1}}{a_{1}}\right)^{1-1}=\left(\frac{\xi_{1}}{a_{1}}\right)^{0}=1$

Como esta formulação é análoga à formulação para fissuras de borda, torna-se fácil a identificação do sistema linear, expresso em (5.18), que fornece a solução do Método da Partição: 


$$
\begin{gathered}
{\left[\begin{array}{cc}
-\int_{0}^{a 1} Q_{1} Q_{1} \partial \xi & 0 \\
0 & -\int_{0}^{a 1} Q_{1} Q_{1} \partial \xi
\end{array}\right] \cdot\left\{\begin{array}{l}
\alpha_{1} \\
\alpha_{2}
\end{array}\right\}=\left\{\begin{array}{l}
-\int_{0}^{a 1} b_{1,1} Q_{1} Q_{1} \partial \xi \\
-\int_{0}^{a 1} b_{1,1} Q_{1} Q_{1} \partial \xi
\end{array}\right\} \Rightarrow} \\
\Rightarrow \quad\left[\begin{array}{cc}
-1 & 0 \\
0 & -1
\end{array}\right] \cdot\left\{\begin{array}{l}
\alpha_{1} \\
\alpha_{2}
\end{array}\right\}=\left\{\begin{array}{l}
-1 \\
-1
\end{array}\right\} \Rightarrow\left\{\begin{array}{l}
\alpha_{1} \\
\alpha_{2}
\end{array}\right\}=\left\{\begin{array}{l}
1 \\
1
\end{array}\right\}
\end{gathered}
$$

O fator de intensidade do subproblema local obtido mediante analise numérica através do programa ANSYS é dado por:

$$
K_{j}^{(i)}=K_{1}^{(1)}=K_{1}^{(2)}=1,815
$$

Desta maneira, obtêm-se os valores de $\mathrm{K}_{\mathrm{I}}$ para as duas pontas da única fissura interna do problema através da equação (5.15):

$$
\begin{aligned}
& K^{(1)}=\sum_{j=1}^{1} \alpha_{1} \cdot K_{1}^{(1)}=1 \cdot 1,815=1,815 \\
& K^{(2)}=\sum_{j=1}^{1} \alpha_{2} \cdot K_{1}^{(2)}=1 \cdot 1,815=1,815
\end{aligned}
$$

O erro da aproximação pode ser expresso da seguinte maneira:

$$
E(\%)=\frac{\left|K_{R E F}-K_{A P R O X}\right|}{K_{R E F}} \cdot 100=\frac{|1,819-1,815|}{1,819} \cdot 100=0,2 \%
$$

Com o resultado obtido confirma a validade da aplicação do Princípio de Bueckner para redefinir um problema contendo fissura interna mediante a resolução de dois subproblemas seguidos de sobreposição. 


\subsubsection{Exemplo 2: Chapa com Uma Fissura - Carregamento Não-Uniforme}

Neste exemplo, reproduzem-se as mesmas características da chapa anterior à exceção do carregamento que é dado pela seguinte equação:

$\sigma=1+\left(\frac{x}{10}\right)^{2}$

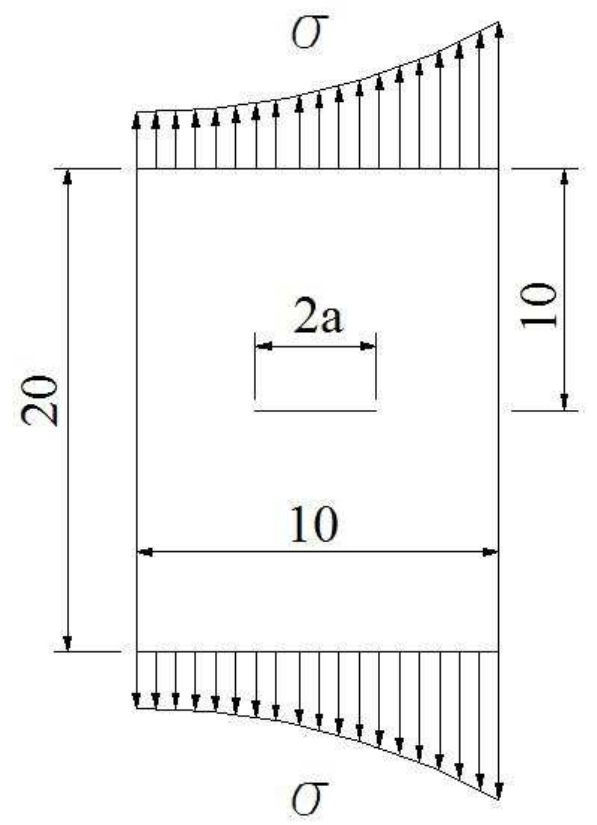

Figura 5.7 - Chapa com 1 fissura e carregamento cte+quadrático

A solução de referência para este problema foi obtida mediante análise por elementos finitos com um alto grau de refinamento de malha na região das pontas de fissura. Os valores dos fatores de intensidade de tensão estão indicados na tabela 5.1 :

Tabela 5.1 - Valores de $K_{I}$ exatos obtidos para carregamento cte+quadrático

\begin{tabular}{|c|c|}
\hline Ponta de Fissura & $\mathbf{K}_{\mathbf{I}}$ \\
\hline 1 (da esquerda) & 2,325 \\
\hline 2 (da direita) & 2,502 \\
\hline
\end{tabular}


Neste exemplo utilizam-se três componentes da base polinomial para a aproximação do campo de tensão na linha da fissura. Assim sendo, os índices para a utilização das equações do Método da Partição ficam definidos por:

$\mathrm{I}=1 \quad\left(\mathrm{n}^{\mathrm{o}}\right.$ de fissuras internas $)$

$\mathrm{J}=3 \quad\left(\mathrm{n}^{\mathrm{o}}\right.$ de termos da aproximação)

$\mathrm{M}=\mathrm{I} . \mathrm{J}=3$

Como neste exemplo utilizam-se três bases aproximativas, torna-se necessário a coleta de valores de tensão em três pontos para cada ponta da fissura interna. Os três pontos que situam-se no eixo $\xi_{1}$, ou seja, no comprimento $\boldsymbol{a}$ adjacente à ponta da fissura do lado esquerdo contribuem para a obtenção dos valores de $\mathrm{K}_{\mathrm{I}}$ desta ponta da fissura. Já os três pontos que situam-se no eixo $\xi_{2}$, ou seja, no comprimento $\boldsymbol{a}$ adjacente à ponta da fissura do lado direito contribuem para a obtenção dos valores de $\mathrm{K}_{\mathrm{I}}$ desta ponta da fissura. Note na figura 5.8 que o ponto número 2 , localizado no extremo dos eixos locais $\xi_{1}$ e $\xi_{2}$ serve de referência para a determinação de $\mathrm{K}_{\mathrm{I}}$ das duas pontas, o contrário ocorre com os pontos de números 1 e 3 que contribuem somente para a determinação de $K_{I}$ pertencente aos seus respectivos eixos.

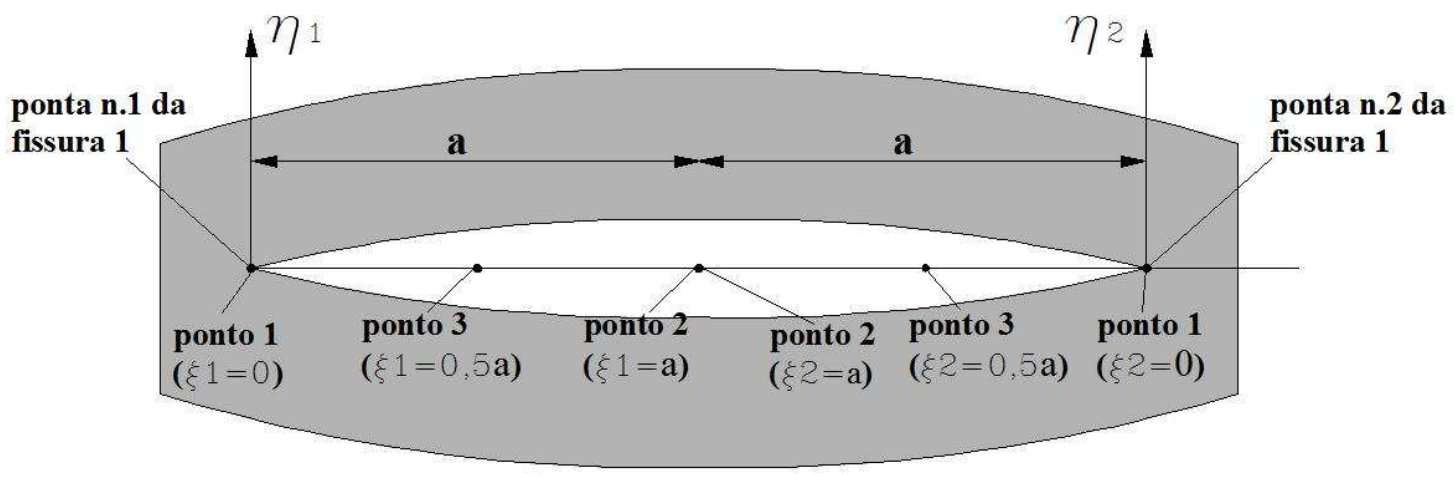

Figura 5.8 - Pontos utilizados para a coleta de valores de tensão.

Os valores aproximados de $\mathrm{K}_{\mathrm{I}}$ para as duas pontas da fissura interna obtidos pelo Método da Partição são dados na tabela 5.2: 
Tabela 5.2 - Valores de $\mathrm{K}_{\mathrm{I}}$ aproximado obtidos para carregamento cte+quadrático

\begin{tabular}{|c|c|c|c|c|c|}
\hline \multicolumn{6}{|c|}{ Carregamento Cte+Quadrático } \\
\hline \multicolumn{3}{|c|}{ Ponta de Fissura 1: $K_{\mathrm{REF}}=2,325$} & \multicolumn{3}{|c|}{ Ponta de Fissura 2: $K_{\mathrm{REF}}=2,502$} \\
\hline $\begin{array}{c}\mathrm{N}^{\circ} \text { termos } \\
\text { Aprox. }\end{array}$ & $\overline{\mathbf{K}_{\text {APROX }}}$ & $\mathrm{E}(\%)$ & $\begin{array}{c}\mathrm{N}^{\mathbf{0}} \text { termos } \\
\text { Aprox. }\end{array}$ & $\overline{\mathbf{K}_{\text {APROX }}}$ & $\mathbf{E}(\%)$ \\
\hline 1 & 2,23 & 4,1 & 1 & 2,60 & 3,91 \\
\hline 2 & 2,308 & 0,71 & 2 & 2,538 & 1,43 \\
\hline 3 & 2,308 & 0,71 & 3 & 2,531 & 1,30 \\
\hline
\end{tabular}

Com os resultados obtidos fica constatada a eficiência do Método da Partição no que diz respeito à obtenção de $\mathrm{K}_{\mathrm{I}}$ para problemas contemplando carregamentos com variações polinomiais.

\subsubsection{Exemplo 4: Chapa com Duas Fissuras - Carregamento Não-Uniforme}

Neste exemplo, ilustrado na figura 5.9, considera-se uma chapa contendo duas fissuras $(2 a=2)$ com as dimensões abaixo sendo submetida a um carregamento idêntico ao do exemplo anterior (carregamento constante + quadrático). As propriedades elásticas do material da chapa também se mantêm as mesmas: $G=1$ e $v=0,3$.

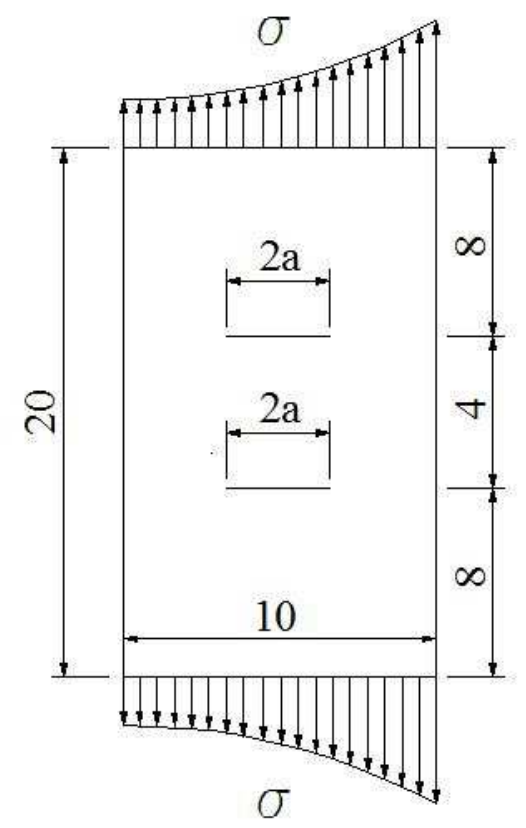

Figura 5.9 - Chapa com 2 fissuras e carregamento cte+quadrático 
Como no exemplo anterior, a solução de referência deste problema foi obtida mediante análise em elementos finitos com um alto grau de refinamento de malha na região das pontas das duas fissuras internas. Os valores obtidos estão apresentados na tabela 5.3:

Tabela 5.3 - Valores-Referência de $K_{I}$ para 2 fissuras internas e carregamento cte+quadrático

\begin{tabular}{|c|c|c|c|c|c|}
\hline \multicolumn{6}{|c|}{ Carregamento Cte+Quadrático } \\
\hline \multicolumn{2}{|c|}{ Fissura Interna 1 (Superior) } & \multicolumn{2}{c|}{ Fissura Interna 2 (Inferior) } \\
\hline $\begin{array}{c}\mathbf{N}^{\mathbf{0}} \text { da Ponta } \\
\text { de Fissura }\end{array}$ & Localização & K $_{\text {APRox }}$ & $\begin{array}{c}\mathbf{N}^{\mathbf{0}} \text { da Ponta } \\
\text { de Fissura }\end{array}$ & Localização & K $_{\text {APRox }}$ \\
\hline 1 & Esquerda & 2,148 & 3 & Esquerda & 2,148 \\
\hline 2 & Direita & 2,414 & 4 & Direita & 2,414 \\
\hline
\end{tabular}

Empregando, novamente, três termos da aproximação, os valores aproximados de $\mathrm{K}_{\mathrm{I}}$ determinados pelo Método da Partição para as duas fissuras internas são dados na tabela 5.4:

Tabela 5.4 - Valores de $K_{I}$ aproximados obtidos para 2 fissuras internas e carregamento cte+quadrático

\begin{tabular}{|c|c|c|c|c|c|}
\hline \multicolumn{6}{|c|}{ Carregamento Cte+Quadrático } \\
\hline \multicolumn{3}{|c|}{$\begin{array}{c}\text { Pontas de Fissura } N^{o s} 1 \text { e } 3 \\
\left(K_{\mathrm{REF}}=2.148\right)\end{array}$} & \multicolumn{3}{|c|}{$\begin{array}{c}\text { Pontas de Fissura } N^{o s} 2 \text { e } 4 \\
\left(K_{\mathrm{REF}}=2.414\right)\end{array}$} \\
\hline $\mathrm{N}^{0}$ termos Aprox. & $\mathbf{K}_{\text {APROX }}$ & $\mathrm{E}(\%)$ & $\mathrm{N}^{\circ}$ termos Aprox. & $\mathbf{K}_{\text {APROX }}$ & $\mathrm{E}(\%)$ \\
\hline 1 & 2,133 & 1,7 & 1 & 2,479 & 2,7 \\
\hline 2 & 2,179 & 1,44 & 2 & 2,400 & 0,58 \\
\hline 3 & 2,17 & 1,02 & 3 & 2,402 & 0,49 \\
\hline
\end{tabular}

Os resultados obtidos confirmam a eficiência do Método da Partição no que diz respeito à obtenção de $\mathrm{K}_{\mathrm{I}}$ para problemas contendo mais de uma fissura interna e que possuam carregamentos com variações polinomiais. 


\subsubsection{Exemplo 6: Chapa com Duas Fissuras - Carregamento Uniformemente Distribuído}

Neste exemplo, ilustrado na figura 5.10, considera-se uma chapa com dimensões 10x60 ( $\mathrm{W}=10$ e $\mathrm{H}=60$ ) contendo duas fissuras $(2 a=2)$ e submetida a um carregamento cisalhante constante $(\tau=1)$. As propriedades elásticas do material da chapa são: $G=1$ e $v=$ 0,3 .

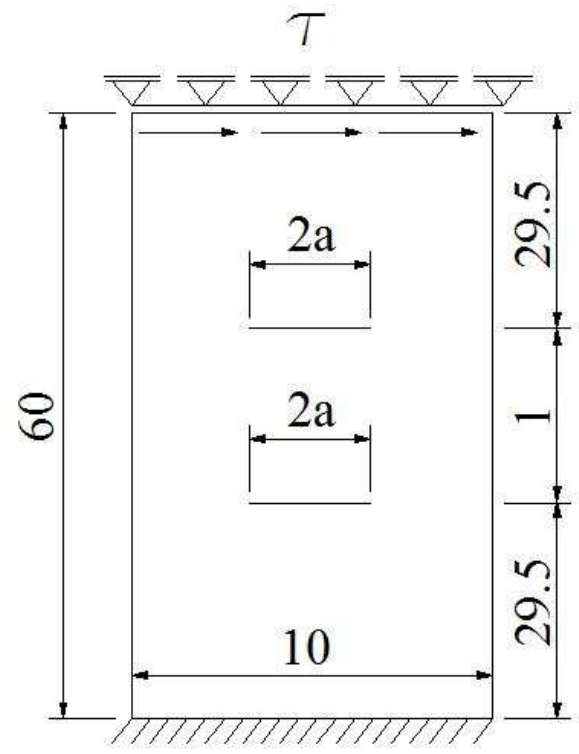

Figura 5.10 - Chapa com 2 fissuras e carregamento cisalhante constante

A solução de referência deste problema foi obtida mediante análise em elementos finitos com um alto grau de refinamento de malha na região das pontas da fissura. Os valores dos fatores de intensidade de tensão estão apresentados na tabela 5.5:

Tabela 5.5 - Valores-Referênciade $K_{\text {II }}$ para carregamento cisalhante constante

\begin{tabular}{|c|c|}
\hline Fissuras Internas 1 e 2 & $\mathbf{K}_{\mathbf{I I}}$ \\
\hline Pontas de Fissura 1, 2, 3 e 4 & 1,654 \\
\hline
\end{tabular}

Como o carregamento cisalhante está aplicado em uma distância considerável em relação às fissuras, o efeito deste nas linhas de fissura do subproblema $P_{G}^{(0)}$ é o de um cisalhamento constante. Sendo assim, considera-se apenas um termo da base de aproximação (constante) na resolução pelo Método da Partição: 
$Q_{j}\left(\xi_{i} / a_{i}\right)=\left(\frac{\xi_{i}}{a_{i}}\right)^{j-1} \quad \Rightarrow \quad Q_{1}\left(\xi_{1} / a_{1}\right)=\left(\frac{\xi_{1}}{a_{1}}\right)^{1-1}=\left(\frac{\xi_{1}}{a_{1}}\right)^{0}=1$

A solução do sistema linear [IG]. $\{\alpha\}=\{r\}$, dado pelo desenvolvimento da equação (5.18) , resulta nos fatores de escala que proporcionam a solução do problema. Os valores obtidos para $\mathrm{K}_{\mathrm{II}}$ estão apresentados na tabela 5.6 :

Tabela 5.6 - Valores de $\mathrm{K}_{\mathrm{II}}$ aproximados obtidos para carregamento cisalhante constante

\begin{tabular}{|c|c|c|}
\hline $\mathbf{N}^{\mathbf{o}}$ termos Aprox. & $\mathbf{K}_{\text {APROx }}$ & $\mathbf{E}(\%)$ \\
\hline 1 & 1,632 & 1,34 \\
\hline
\end{tabular}

Os exemplos demonstrados nos últimos tópicos provam a capacidade do Método da Partição na obtenção de fatores de intensidade de tensão em análises de problemas que contenham interações entre fissuras internas. Importante destacar que as respostas foram obtidas com convergência exponencial e com menos precisão (em torno de 0,5 a $1 \%$ ) do que aquelas obtidas em casos contendo apenas fissuras de borda. Note que em todos os exemplos realizados para as duas formulações (fissuras de borda e internas) a largura das chapas se manteve de mesmo valor $(\mathrm{W}=10)$ e o comprimento das fissuras de borda são a metade do comprimento $(1,0)$ das fissuras internas $(2,0)$. Sendo assim, em se tratando de problemas com comportamentos semelhantes, deduz-se que, quanto maior for o comprimento da fissura, mais difícil se torna realizar a aproximação do campo de tensões nestas linhas de fissura. Tal fato se deve à variação das tensões crescerem dentro deste comprimento, tornando-se uma distribuição de tensão mais complexa e conseqüentemente mais difícil de ser reproduzida por uma base de funções polinomiais. Sendo assim, tal dificuldade em representar este campo de tensões torna a resposta menos precisa. 


\subsection{O Emprego de Funções Racionais na Aproximação de Casos Contendo Fissuras-Furo}

\subsubsection{Exemplo 1: Chapa com Uma Fissura-Furo - Carregamento Uniformemente Distribuído - Modo I de Abertura}

Tendo em vista a aplicação do Método da Partição a situações mais específicas como na análise de rupturas em carenagens de aeronaves, o seguinte exemplo tem como objetivo a determinação do fator de intensidade de tensão em uma fissura contida na vizinhança de um furo, retratando o problema de fratura em região rebitada.

Neste exemplo, considera-se uma chapa com dimensões 10x20 (W=10 e H=20), comprimento de fissura $a=1$, raio do furo $r=1,5$ e tensão constante unitária $(\sigma=1)$ aplicada ao longo das faces inferior e superior da chapa, conforme ilustra a figura 5.11:

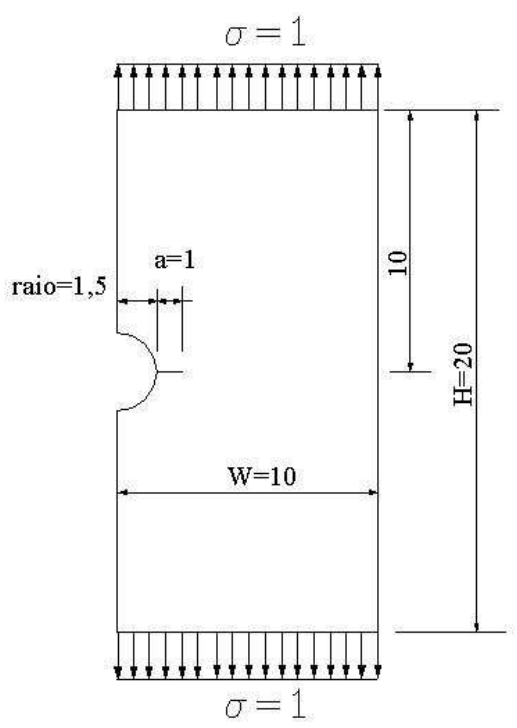

Figura 5.11 - Chapa com 1 furo-fissura

Os dados do problema são os seguintes:

a) Propriedades do material:

$$
\begin{aligned}
& G=1,0 \\
& v=0,3
\end{aligned}
$$


b) Características da simulação para a determinação do valor de referência para o fator de intensidade de tensão:

$\checkmark$ Eixo de simetria horizontal

$\checkmark$ Elemento finito quadrático de quatro nós (deslocamentos em duas direções por nó)

$\checkmark \quad \mathrm{N}^{\mathrm{o}}$ de nós $=15865$

$\checkmark \quad \mathrm{N}^{\mathrm{o}}$ de Elementos $=15749$

$\checkmark$ Refinamento excessivo na ponta da fissura

$\checkmark$ Malha não-estruturada

c) Discretização para os subproblemas $P_{G}^{(0)}$ e $P_{G}^{(k)}$

$\checkmark$ Eixo de simetria horizontal

$\checkmark \quad \mathrm{N}^{\mathrm{o}}$ de nós $=2087$

$\checkmark \quad \mathrm{N}^{\circ}$ de Elementos $=1996$

$\checkmark$ Sem refinamento excessivo

$\checkmark$ Malha não-estruturada

d) Discretização para o subproblema $P_{L}^{(k)}$

$\checkmark$ Eixo de simetria horizontal

$\checkmark \quad \mathrm{N}^{\mathrm{o}}$ de nós $=15865$

$\checkmark \quad \mathrm{N}^{\mathrm{o}}$ de Elementos $=15749$

$\checkmark$ Sem refinamento excessivo

$\checkmark$ Malha não-estruturada

e) O valor de referência do fator de intensidade de tensão está mostrado na tabela 5.7.

Tabela 5.7 - Valor de K para o exemplo contendo 1 furo-fissura

\begin{tabular}{|c|c|}
\hline Fissura & $\mathbf{K}_{\mathbf{I}}$ \\
\hline 1 & 4,110 \\
\hline
\end{tabular}


d) Parâmetros que definem a somatória do Método da Partição:

$\mathrm{n}^{\circ}$ de termos da aproximação adotados $=5 \quad \Rightarrow \quad \mathrm{J}=5$

$\mathrm{n}^{\mathrm{o}}$ de fissuras $=1 \quad \Rightarrow \quad \mathrm{I}=1$

$\mathrm{M}=\mathrm{J} \times \mathrm{I}=5 \times 1=5$

Um ponto importante na análise de problemas envolvendo o conjunto furo-fissura refere-se ao fato de que a presença do furo acarreta em uma menor regularidade no campo de tensões do subproblema $P_{G}^{(0)}$, particularmente na linha da fissura. Isto faz com que a base aproximativa polinomial necessite de muitos termos para capturar a solução exata do problema ( $K_{I}$ ). Sendo assim, Babuska e Andersson (2005) definem o espaço das funções que atendem a este tipo de problema do seguinte modo:

$L_{\alpha_{R}, \beta_{R}}(f i s s)=\left\{R \mid \int_{\text {fiss }} R^{2}(\xi) \cdot(1-\xi)^{\alpha_{R}}(1+\xi)^{\beta_{R}} \partial \xi=\|R\|_{\alpha_{\alpha_{R} \beta_{R}(f i s)}^{2}}^{2}<\infty,-1<\alpha_{R}, \beta_{R}\right\}$

e com base em testes realizados utilizando o Método da Partição para resolução de problemas envolvendo o conjunto furo-fissura, aqueles autores recomendam os seguintes valores para $\alpha_{R}$ e $\beta_{R}$ :

$$
\left\{\begin{array}{l}
\alpha_{R}=0 \\
\beta_{R}=-\frac{1}{2}
\end{array}\right.
$$

Sendo assim, obtêm-se a nova base de funções aproximativas em (5.39) para problemas dessa natureza. Outro trabalho que apresenta resultados neste sentido é dado por Burns, Glinka e Kiciak (2003).

$$
R_{j}\left(\xi_{i} / a_{i}\right)=\frac{1}{\sqrt{1+\xi_{i}}} \cdot\left(\frac{\xi_{i}}{a_{i}}\right)^{j-1}
$$

As figuras 5.12 e 5.13 ilustram as distribuições de tensão $\left(\sigma_{y}\right)$ referentes aos subproblemas $P_{G}^{(0)}$ e $P_{L}^{(k)}$, respectivamente, deste exemplo do Método da Partição: 


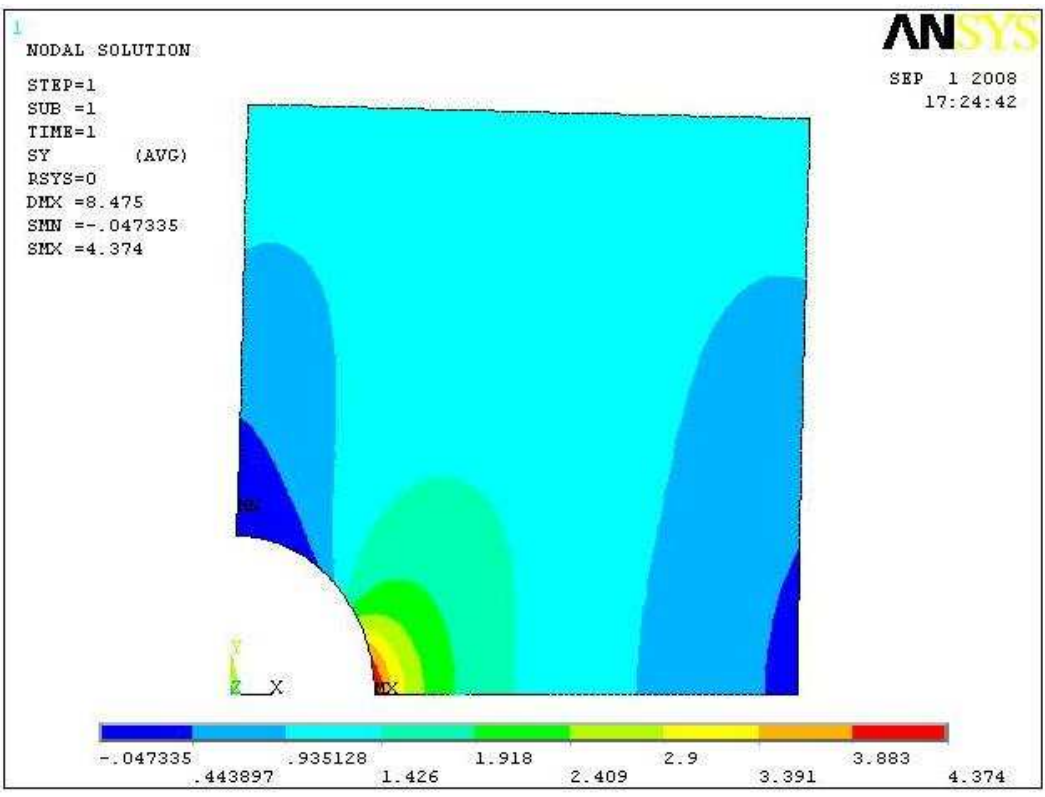

Figura 5.12 - Campo de tensões $\left(\sigma_{y}\right)$ para o subproblema $P_{G}^{(0)}$

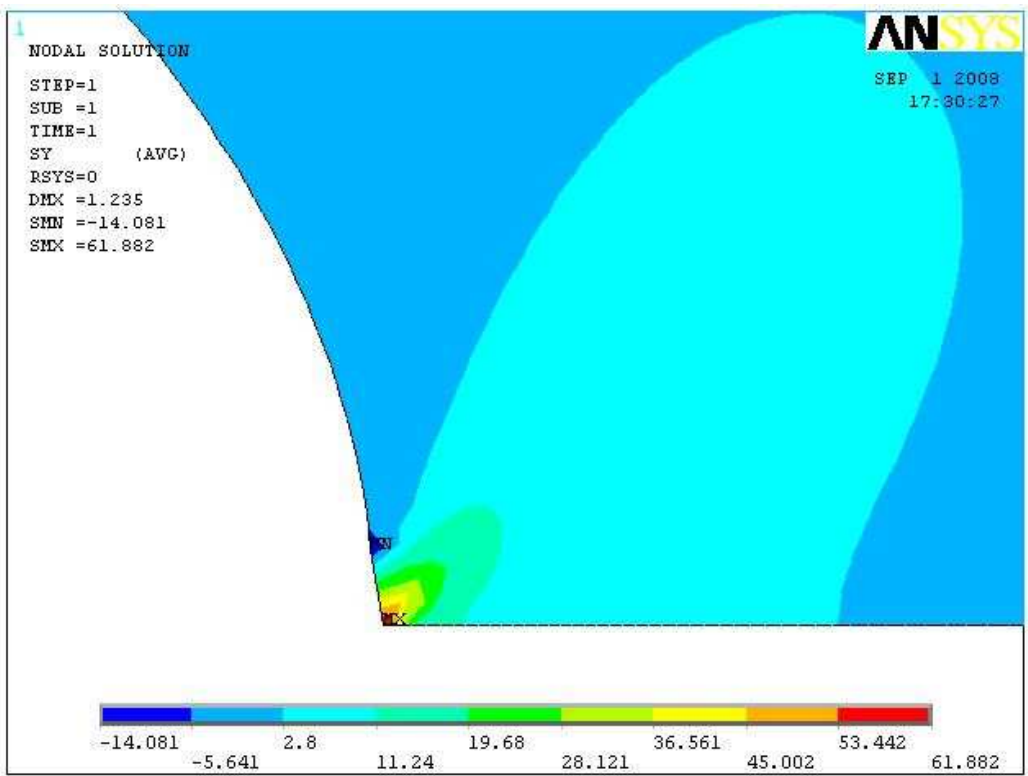

Figura 5.13 - Campo de tensões $\left(\sigma_{y}\right)$ na ponta da fissura - Subproblema $P_{L}^{(k=1)}$

Os resultados obtidos para a determinação do fator de intensidade de tensão (modo I de abertura) são os descritos na tabela 5.8: 
Tabela 5.8 - Valores-Referência de $K_{I}$ para o exemplo contendo 1 furo-fissura

\begin{tabular}{|c|c|c|}
\hline \multicolumn{3}{|c|}{ Conjunto Furo-fissura: $\mathrm{K}_{\mathrm{REF}}=4,110$} \\
\hline $\mathrm{N}^{\mathrm{o}}$ termos Aprox. & $\mathrm{K}_{\mathrm{APROX}}$ & $\mathrm{E}(\%)$ \\
\hline 1 & 2,672 & 34,00 \\
\hline 2 & 4,737 & 15,25 \\
\hline 3 & 4,029 & 1,97 \\
\hline 5 & 4,104 & 0,15 \\
\hline
\end{tabular}

Observando os resultados, percebe-se novamente a convergência exponencial do método e um número de termos maior do que aqueles apresentados nos problemas que não envolvem a presença de furos adjacentes à fissura. A precisão da resposta com dois termos a mais mantêm a precisão da resposta com um erro inferior a $0,5 \%$.

\subsubsection{Exemplo 2: Chapa com Duas Fissuras-Furo - Carregamento Uniformemente Distribuído - Modo I de Abertura}

Neste exemplo utiliza-se uma chapa com as mesmas características geométricas do exemplo anterior, sendo adicionado um conjunto furo-fissura na borda lateral da chapa. $\mathrm{O}$ carregamento é mantido constante unitário $(\sigma=1)$. O objetivo deste exemplo é verificar a interação entre fissuras. 


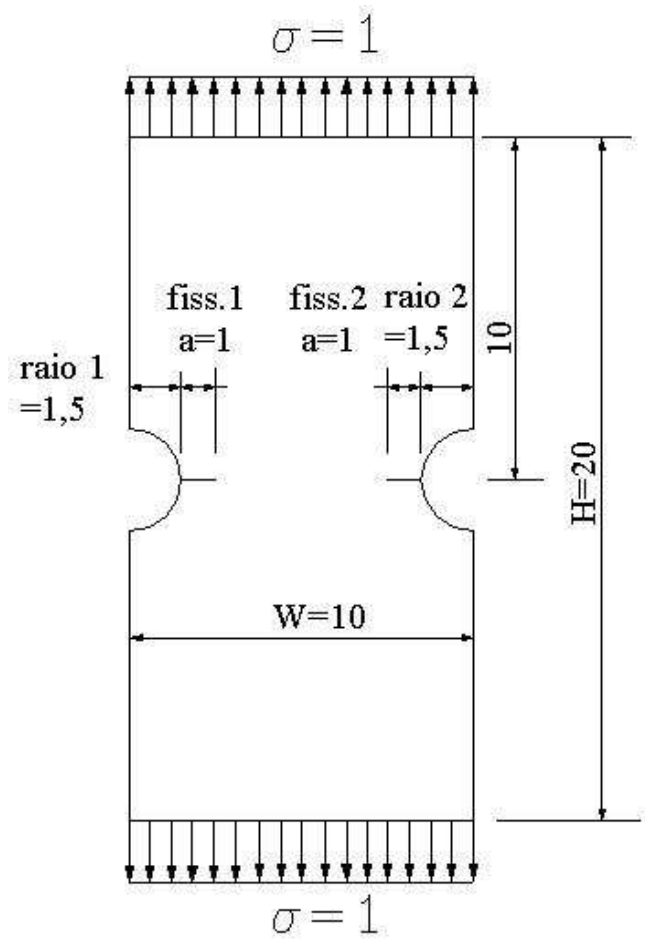

Figura 5.14 - Chapa com furos-fissura

Os dados do problema são os seguintes:

a) Propriedades do material:

$\left\{\begin{array}{l}G=1,0 \\ v=0,3\end{array}\right.$

b) Discretização da simulação para a determinação dos valores de referência dos fatores de intensidade de tensão:

$\checkmark$ Eixo de simetria horizontal

$\checkmark$ Elemento finito quadrático de oito nós (deslocamentos em duas direções por nó)

$\checkmark \quad \mathrm{N}^{\mathrm{o}}$ de nós $=41742$

$\checkmark \quad \mathrm{N}^{\mathrm{o}}$ de Elementos $=13813$

$\checkmark$ Refinamento excessivo na ponta das fissuras

$\checkmark$ Malha não-estruturada

c) Discretização para os subproblemas $P_{G}^{(0)}$ e $P_{G}^{(k)}$

$\checkmark$ Eixo de simetria horizontal

$\checkmark \quad \mathrm{N}^{\mathrm{o}}$ de nós $=6936$ 
$\checkmark \quad \mathrm{N}^{\mathrm{o}}$ de Elementos $=2245$

$\checkmark$ Sem refinamento excessivo

$\checkmark$ Malha não-estruturada

d) Malha para o subproblema $P_{L}^{(k)}$

$\checkmark$ Eixo de simetria horizontal

$\checkmark \quad \mathrm{N}^{\mathrm{o}}$ de nós $=35722$

$\checkmark \quad \mathrm{N}^{\circ}$ de Elementos $=9469$

$\checkmark$ Com refinamento na região da ponta de fissura.

$\checkmark$ Malha não-estruturada

A figura 5.15 mostra o detalhe do contorno auxiliar $\Gamma^{(i)}$ definido no entorno do conjunto furo-fissura. Note que as dimensões da chapa $(10$ x 10) são de uma ordem de grandeza próxima à do comprimento da fissura $(1,0)$. Com isto, adotou-se o contorno infinito com dimensões idênticas às do contorno do problema original, por isso a presença do furo no lado direito da chapa, porém ausente da fissura.

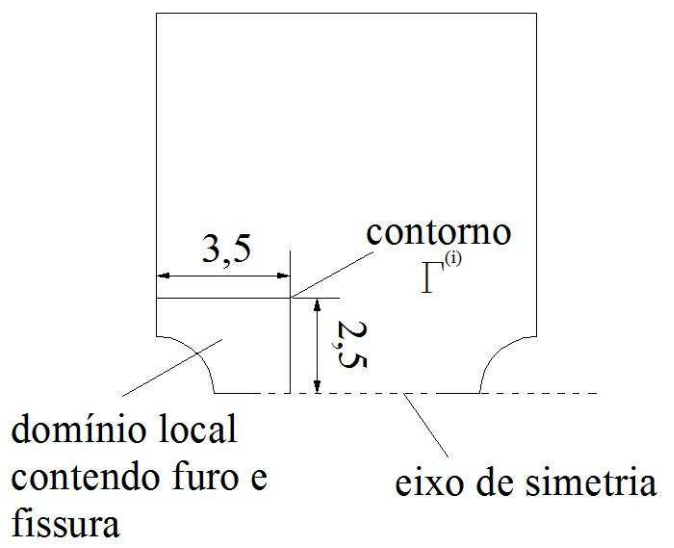

Figura 5.15 - Delimitação do domínio local de $P_{L}^{(k)}$

e) Na tabela 5.9 reúnem-se os valores de referência dos fatores de intensidade de tensão:

Tabela 5.9 - Valores de K para o exemplo com 2 furos-fissura

\begin{tabular}{|c|c|}
\hline Fissura & $\mathbf{K}_{\mathbf{I}}$ \\
\hline 1 & 3,275 \\
\hline 2 & 3,275 \\
\hline
\end{tabular}


d) Os parâmetros para as somatórias do Método da Partição são dados por:

$\mathrm{n}^{\mathrm{o}}$ de termos da aproximação adotados $=5 \quad \Rightarrow \quad \mathrm{J}=5$

$\mathrm{n}^{\mathrm{o}}$ de fissuras $=2 \quad \Rightarrow \quad \mathrm{I}=2$

$\mathrm{M}=\mathrm{J} \times \mathrm{I}=5 \times 2=10$

Os resultados obtidos para a determinação dos fatores de intensidade de tensão são mostrados na tabela 5.10:

Tabela 5.10 - Valores-Referência de K para o exemplo contendo 2 furos-fissura

\begin{tabular}{|c|c|c|c|c|c|}
\hline \multicolumn{5}{|c|}{ Carregamento Cte } \\
\hline \multicolumn{3}{|c|}{ Fissura 1: KREF $=3,275$} & \multicolumn{3}{c|}{ Fissura 2: K } \\
\hline $\begin{array}{c}\mathbf{N}^{\mathbf{0}} \text { termos } \\
\text { Aprox. }\end{array}$ & $\mathbf{K}_{\text {APROx }}$ & $\mathbf{E}(\%)$ & $\begin{array}{c}\mathbf{N}^{\mathbf{0}} \text { termos } \\
\text { Aprox. }\end{array}$ & $\mathbf{K}_{\text {APROx }}$ & $\mathbf{E}(\%)$ \\
\hline 1 & 2,418 & 26,16 & 1 & 2,418 & 26,16 \\
\hline 2 & 3,6689 & 12,17 & 2 & 3,6689 & 12,17 \\
\hline 3 & 3,216 & 1,79 & 3 & 3,253 & 0,66 \\
\hline 5 & 3,2714 & 0,11 & 5 & 3,272 & 0,09 \\
\hline
\end{tabular}

Nota-se que em relação aos resultados obtidos no exemplo anterior, o número de termos de aproximação necessários para uma resposta com erro inferior a 0,5\% mantêm-se em cinco, sendo que, com três termos de aproximação tem-se de antemão uma boa aproximação do valor do resultado final. Com este exemplo confirma-se a capacidade do método na análise de múltiplas fissuras mesmo com a presença de campos de tensões mais complexos bastando apenas utilizar-se a função aproximativa adequada para este campo de tensões. Desta maneira o método pode ser utilizado para análises de maior escala no que se diz respeito ao número de fissuras, o que vem a ser uma ferramenta bastante útil nos testes de componentes de aeronaves (chapas rebitadas). 


\subsection{Combinação de casos envolvendo fissura interna, de borda e borda- fissura.}

Os exemplos que seguem tem por objetivo propor uma metodologia para a montagem do sistema linear $[\mathrm{IG}] .\{\alpha\}=\{\mathrm{r}\}$ para casos que envolvam a presença de fissuras de diferentes formas, dentre elas citam-se: fissuras internas, fissuras de borda e fissuras na borda de furos. As análises são realizadas considerando-se somente o modo I de abertura, porém a metodologia pode ser estendida aos demais modos de abertura. No primeiro caso realiza-se uma análise de chapa contendo fissuras internas e fissuras de borda. A meta principal desta análise refere-se à montagem do referido sistema linear além da verificação da precisão da resposta em termos de fatores de intensidade de tensão. $\mathrm{O}$ outro caso trata da análise de uma chapa contendo duas fissuras, sendo uma de borda e a outra de borda-furo. A meta neste caso é a verificação da melhor função aproximativa a utilizar.

\subsubsection{Exemplo 1: Chapa Contendo Fissuras Internas e de Borda - Carregamento Uniformemente Distribuído}

Neste exemplo considera-se uma chapa com dimensões $12 \times 12(\mathrm{~W}=12$ e $\mathrm{H}=12)$, comprimento de fissuras internas $2 \mathrm{a}=2$, comprimento de fissuras de borda $\mathrm{a}=1$ e tensão vertical constante unitária $(\sigma=1)$ aplicada ao longo das faces inferior e superior da chapa. São explorados os eixos de simetria tanto vertical quanto horizontal. As figuras 5.16 e 5.17 ilustram o exemplo em questão: 


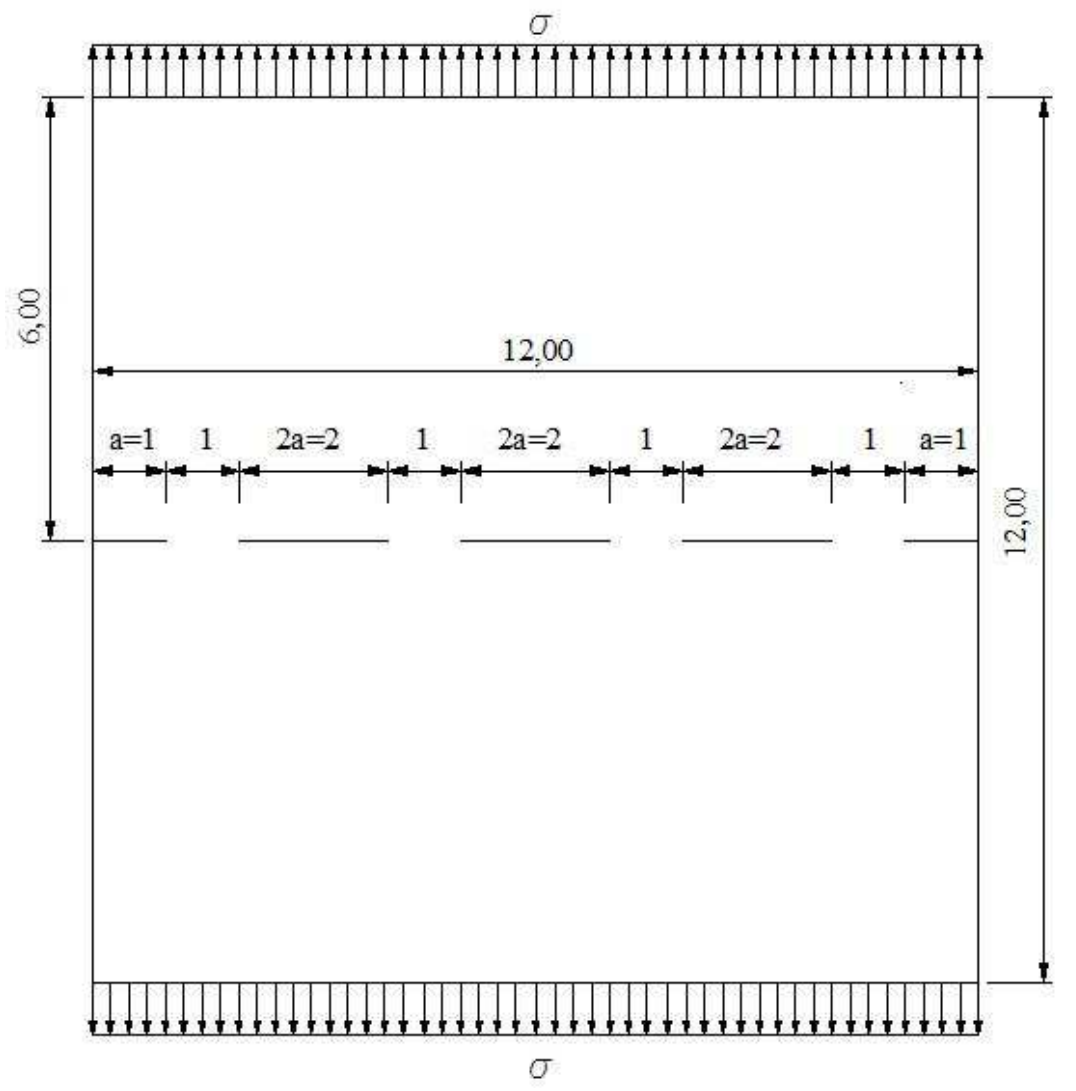

Figura 5.16 - Chapa com 3 fissuras internas e 2 fissuras de borda com carregamento constante

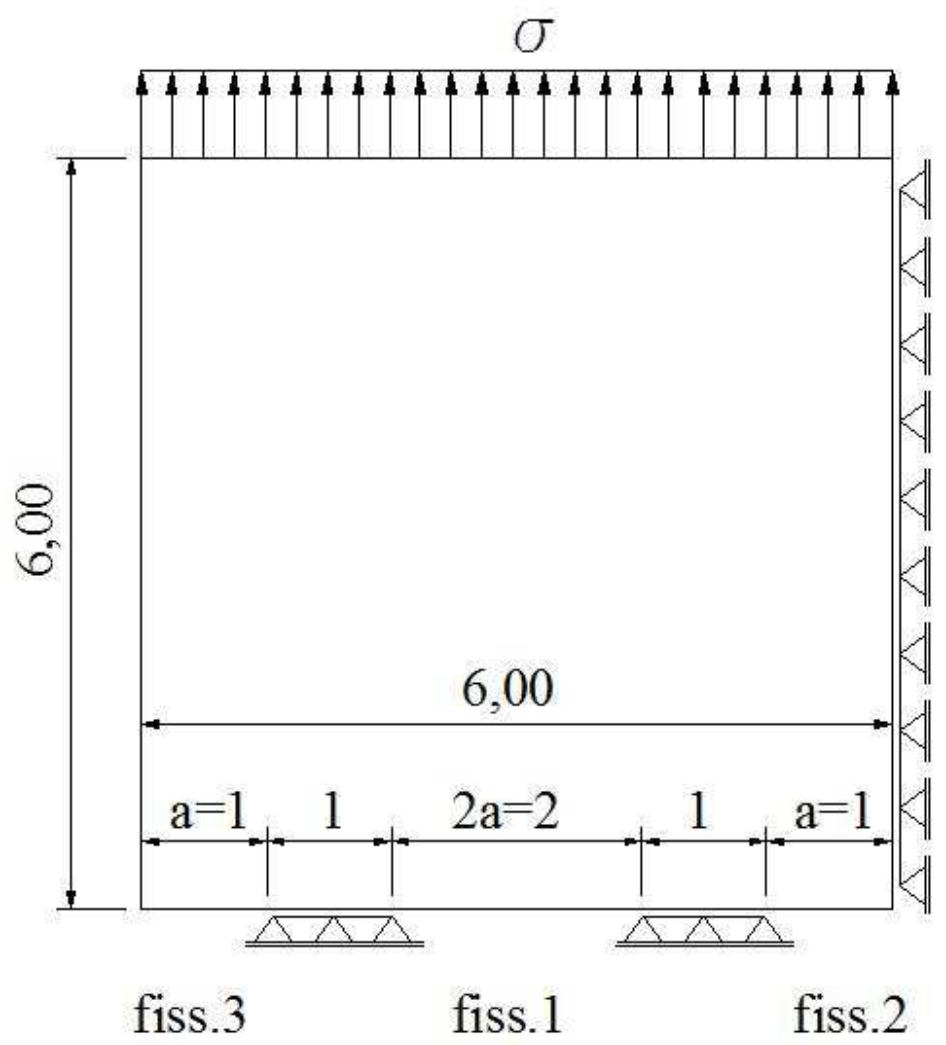

Figura 5.17 - 1/4 da chapa devido à utilização dos eixos de simetria horizontal e vertical 
A solução de referência deste problema foi obtida mediante análise em elementos finitos com um alto grau de refinamento de malha na região das pontas de fissura. Os valores obtidos para os fatores de intensidade de tensão estão mostrados na tabela 5.11:

Tabela 5.11 - Valores-Referência de $K_{I}$ para chapa contendo 1 fissura interna e 2 de borda para carregamento constante

\begin{tabular}{|c|c|}
\hline Fissura & $\mathbf{K}_{\mathbf{I}}$ \\
\hline 1 (valor para as duas pontas de fissura) & 2,258 \\
\hline 2 (borda - direita) & 2,255 \\
\hline 3 (borda - esquerda) & 2,390 \\
\hline
\end{tabular}

Sendo o carregamento constante, utiliza-se somente um termo de aproximação sendo os índices para a utilização das equações do Método definidos por:

$\mathrm{I}=4 \quad$ (n' de "pontas" de fissuras)

$\mathrm{J}=1 \quad\left(\mathrm{n}^{\mathrm{o}}\right.$ de termos da aproximação)

$\mathrm{M}=\mathrm{I} . \mathrm{J}=4$

Desta maneira, o sistema linear $[\mathrm{IG}] .\{\alpha\}=\{\mathrm{r}\}$ que expressa a solução do problema é dado da seguinte forma:

$[I G] \cdot\{\alpha\}=\{r\} \quad \Rightarrow$

$$
\left[\begin{array}{cccc}
-\int_{0}^{a 1} Q_{1} Q_{1} \partial \xi & 0 & \int_{0}^{a 1} c_{1,1}^{(3)} Q_{1} Q_{1} \partial \xi & \int_{0}^{a 1} c_{1,1}^{(4)} Q_{1} Q_{1} \partial \xi \\
0 & -\int_{0}^{a 1} Q_{1} Q_{1} \partial \xi & \int_{0}^{a 1} c_{2,1}^{(3)} Q_{1} Q_{1} \partial \xi & \int_{0}^{a 1} c_{2,1}^{(4)} Q_{1} Q_{1} \partial \xi \\
\int_{0}^{a 2} c_{3,1}^{(1)} Q_{1} Q_{1} \partial \xi & \int_{0}^{a 2} c_{3,1}^{(2)} Q_{1} Q_{1} \partial \xi & -\int_{0}^{a 2} Q_{1} Q_{1} \partial \xi & \int_{0}^{a 2} c_{3,1}^{(4)} Q_{1} Q_{1} \partial \xi \\
\int_{0}^{a 3} c_{4,1}^{(1)} Q_{1} Q_{1} \partial \xi & \int_{0}^{a 3} c_{4,1}^{(2)} Q_{1} Q_{1} \partial \xi & \int_{0}^{a 3} c_{4,1}^{(3)} Q_{1} Q_{1} \partial \xi & -\int_{0}^{a 3} Q_{1} Q_{1} \partial \xi
\end{array}\right] \cdot\left\{\begin{array}{l}
\alpha_{1} \\
\alpha_{2} \\
\alpha_{3} \\
\alpha_{4}
\end{array}\right\}=-\left\{\begin{array}{l}
\int_{0}^{a 1} b_{1,1} Q_{1} Q_{1} \partial \xi \\
\int_{0}^{a 1} b_{2,1} Q_{1} Q_{1} \partial \xi \\
\int_{0}^{a 2} b_{3,1} Q_{1} Q_{1} \partial \xi \\
\int_{0}^{a 3} \\
\int_{0}^{a 3} b_{4,1} Q_{1} Q_{1} \partial \xi
\end{array}\right\}
$$


Importante ressaltar que cada linha da matriz [IG] representa uma linha de fissura. A linha 1 representa o primeiro semi-comprimento da fissura interna número 1 , ou seja, o comprimento a do lado esquerdo. A linha 2 da mesma matriz representa o segundo semicomprimento da fissura interna número 1, ou seja, o comprimento a do lado direito. A linha 3 representa a linha de fissura de borda situada na extremidade direita da chapa e a linha 4 representa a linha de fissura de borda situada na extremidade esquerda da chapa.

Definidas as posições onde se situam as linhas de cada fissura, o próximo passo referese à definição dos carregamentos $\boldsymbol{k}$ que produzirão efeitos em termos de tensões nestas mesmas linhas de fissura. Sendo que a matriz [IG] possui tanto os efeitos dos subproblemas locais $P_{L}^{(k)}$ quanto dos subproblemas globais $P_{G}^{(k)}$, torna-se fácil a verificação dos efeitos dos subproblemas locais $P_{L}^{(k)}$ que são dados na diagonal principal da mesma. Assim sendo, os elementos $\mathrm{IG}_{1,1}, \mathrm{IG}_{2,2}, \mathrm{IG}_{3,3}$ e $\mathrm{IG}_{4,4}$ representam as tensões devido o carregamento aproximativo $Q_{1}$ (de compressão) aplicado nas faces da fissura interna (semi-comprimentos esquerdo e depois direito), da fissura de borda do lado direito e da fissura de borda do lado esquerdo, respectivamente.

Uma vez definidos os efeitos locais, restam definir as componentes responsáveis por realizar a interação entre fissuras. Entretanto, alguns esclarecimentos tornam-se necessários. O primeiro é que os termos de aproximação $c_{i, j}^{(k)}$ coincidem com o próprio valor da tensão $t_{G}^{(k)}$ em uma dada linha de fissura, pois ao se fazer a combinação linear para apenas um termo de aproximação têm-se que:

$t_{G}^{(k)}=c_{i, j}^{(k)} \cdot Q_{i}\left(\xi_{i} / a_{i}\right) \quad \Rightarrow \quad t_{G}^{(k)}=c_{i, j}^{(k)} \cdot 1 \quad \Rightarrow \quad t_{G}^{(k)}=c_{i, j}^{(k)}$

Outro aspecto importante refere-se aos índices $\boldsymbol{k}$, que valem 1 para o carregamento $Q_{1}$ aplicado no primeiro semi-comprimento $\boldsymbol{a}$ da fissura interna, 2 para o carregamento $Q_{1}$ aplicado no segundo semi-comprimento $\boldsymbol{a}$ da mesma fissura interna, 3 para o carregamento $Q_{1}$ aplicado na fissura de borda da extremidade direita da chapa e 4 para o carregamento $Q_{1}$ aplicado na fissura de borda da extremidade esquerda da chapa. Desta maneira, fica fácil notar que cada coluna da matriz [IG] representa a influência de um determinado subproblema local sobre as demais linhas de fissura. Porém, nota-se que os elementos $\mathrm{IG}_{1,2}$ e $\mathrm{IG}_{2,1}$ são nulos. Tal fato ocorre devido aos dois semi-comprimentos da fissura interna (que definem duas pontas de fissura) estarem situados dentro de um mesmo domínio $\Gamma^{(i)}$ e a influência de uma 
ponta de fissura para a outra já está contida nos dois primeiros elementos da diagonal principal da referida matriz. Por último, o índice $\boldsymbol{i}$ dos termos $c_{i, j}^{(k)}$ representam as pontas de fissura, ou seja, $\mathrm{i}=1$ representa a primeira ponta da fissura interna $1, \mathrm{i}=2$ representa a segunda ponta da fissura interna $1, \mathrm{i}=3$ representa a ponta da fissura de borda da extremidade direita da chapa e $\mathrm{i}=4$ representa a ponta da fissura de borda da extremidade esquerda da chapa. Sendo assim, os termos que expressam a influência entre fissuras podem ser interpretados da seguinte maneira:

Linha 1 da matriz [IG] , ou seja, primeiro semi-comprimento da fissura interna:

$c_{1,1}^{(3)}$ - tensão oriunda de $Q_{1}$ aplicado na fissura de borda da extremidade direita da chapa, $c_{1,1}^{(4)}$ - tensão oriunda de $Q_{1}$ aplicado na fissura de borda da extremidade esquerda da chapa.

Linha 2 da matriz [IG], ou seja, segundo semi-comprimento da fissura interna: $c_{2,1}^{(3)}$ - tensão oriunda de $Q_{1}$ aplicado na fissura de borda da extremidade direita da chapa, $c_{2,1}^{(4)}$ - tensão oriunda de $Q_{1}$ aplicado na fissura de borda da extremidade esquerda da chapa.

Linha 3 da matriz [IG] , ou seja, fissura de borda da extremidade direita da chapa: $c_{3,1}^{(1)}+c_{3,1}^{(2)}$ - tensão oriunda de $Q_{1}$ aplicado na fissura interna da chapa. (na matriz, cada um destes valores pode ser representado pela metade da influência total) $c_{3,1}^{(4)}$ - tensão oriunda de $Q_{1}$ aplicado na fissura de borda da extremidade esquerda da chapa.

Linha 4 da matriz [IG] , ou seja, fissura de borda da extremidade esquerda da chapa: $c_{4,1}^{(1)}+c_{4,1}^{(2)}$ - tensão oriunda de $Q_{l}$ aplicado na fissura interna da chapa. (na matriz, cada um destes valores pode ser representado pela metade da influência total) $c_{4,1}^{(3)}$ - tensão oriunda de $Q_{l}$ aplicado na fissura de borda da extremidade direita da chapa. Finalmente, o sistema linear $[\mathrm{IG}] .\{\alpha\}=\{\mathrm{r}\}$ fica definido da seguinte forma: 
$\left[\begin{array}{cccc}-1 & 0 & 0,0991 & 0,0727 \\ 0 & -1 & 0,0991 & 0,0727 \\ \frac{0,2025}{2} & \frac{0,2025}{2} & -1 & -0,0119 \\ \frac{0,1249}{2} & \frac{0,1249}{2} & -0,0075 & -1\end{array}\right] \cdot\left\{\begin{array}{l}\alpha_{1} \\ \alpha_{2} \\ \alpha_{3} \\ \alpha_{4}\end{array}\right\}=\left\{\begin{array}{l}-1 \\ -1 \\ -1 \\ -1\end{array}\right\} \Rightarrow\left\{\begin{array}{l}\alpha_{1} \\ \alpha_{3} \\ \alpha_{4}\end{array}\right\}=\left\{\begin{array}{l}1,205 \\ 1,205 \\ 1,230 \\ 1,141\end{array}\right\}$

Os fatores de intensidade de tensão finais, dados pela multiplicação de cada fator $\alpha$ pelo seu respectivo fator de intensidade de tensão local, resultam:

$$
\begin{aligned}
& K^{(1)}=\alpha_{1} \cdot K_{1}^{(1)}=1,205 \cdot 1,882=2,268 \\
& K^{(2)}=\alpha_{2} \cdot K_{1}^{(2)}=1,205 \cdot 1,882=2,268 \\
& K^{(3)}=\alpha_{3} \cdot K_{1}^{(3)}=1,230 \cdot 1,839=2,262 \\
& K^{(4)}=\alpha_{4} \cdot K_{1}^{(4)}=1,141 \cdot 2,057=2,348
\end{aligned}
$$

Os erros de aproximação para cada fissura estão descritas na tabela 5.12:

Tabela 5.12 - Erro da aproximação para os valores de $K_{I}$

\begin{tabular}{|c|c|}
\hline Fissura & $\mathbf{E}(\%)$ \\
\hline 1 (valor para as duas pontas de fissura) & 0,43 \\
\hline 2 (borda - direita) & 0,34 \\
\hline 3 (borda - esquerda) & 1,74 \\
\hline
\end{tabular}

Analisadas as respostas obtidas contata-se que a formulação inicialmente válida somente para fissuras de borda pode ser estendida também para casos contendo os dois tipos de fissuras (interna e de borda), sendo a precisão da resposta similar àquelas apresentadas nos caso contendo somente fissuras internas. 


\subsubsection{Exemplo 2: Chapa Contendo Fissura de Borda e Fissura-Furo - Carregamento Uniformemente Distribuído}

Neste exemplo considera-se uma chapa com dimensões 10x10 (W=10 e $\mathrm{H}=10)$, comprimento de fissura $\mathrm{a}=1$, raio do furo $\mathrm{r}=0,5$ e tensão vertical constante unitária $(\sigma=1)$ aplicada ao longo da face superior da chapa. O exemplo está ilustrado na figura 5.18:

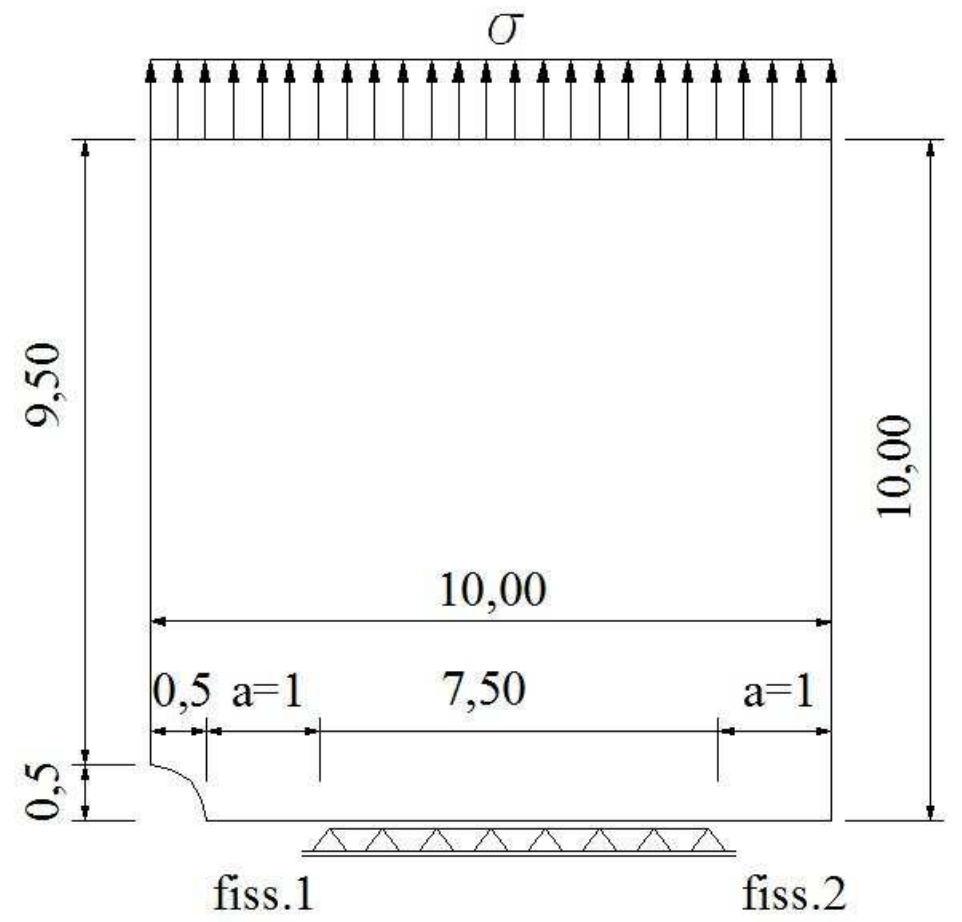

Figura 5.18 - Chapa com 2 fissuras de borda, sendo uma delas na vizinhança de um furo.

A solução de referência deste problema foi obtida mediante análise em elementos finitos com um alto grau de refinamento de malha na região das pontas da fissura. Os valores obtidos estão descritos na tabela 5.13:

Tabela 5.13 - Valores-Referência de $K_{I}$ para chapa com fissura de borda e borda-furo

\begin{tabular}{|c|c|}
\hline Fissura & $\mathbf{K}_{\mathbf{I}}$ \\
\hline Borda-furo (esquerda) & 2,602 \\
\hline Borda (direita) & 1,800 \\
\hline
\end{tabular}


Baseando-se em exemplos anteriores, adotou-se de dois até cinco termos da base de aproximação, sendo os índices para a utilização das equações do Método da Partição definidos por:

$\mathrm{I}=2 \quad\left(\mathrm{n}^{\mathrm{o}}\right.$ de fissuras internas $)$

$\mathrm{J}=2$ até $5 \quad\left(\mathrm{n}^{\mathrm{o}}\right.$ de termos da aproximação)

$\mathrm{M}=\mathrm{I} . \mathrm{J}=2$. (2 até 5$)=4$ até 10

Desta maneira, o sistema linear [IG]. $\{\alpha\}=\{\mathrm{r}\}$ para 2 fissuras e 1 termo de aproximação pode ser expresso da seguinte forma:

$$
[I G] \cdot\{\alpha\}=\{r\} \Rightarrow\left[\begin{array}{ll}
-\int_{0}^{a 1} R_{1} R_{1} \partial \xi & \int_{0}^{a 1} c_{1,1}^{(2)} Q_{1} R_{1} \partial \xi \\
\int_{0}^{a 2} c_{2,1}^{(1)} R_{1} Q_{1} \partial \xi & -\int_{0}^{a 2} Q_{1} Q_{1} \partial \xi
\end{array}\right] \cdot\left\{\begin{array}{l}
\alpha_{1} \\
\alpha_{2}
\end{array}\right\}=\left\{\begin{array}{l}
\int_{0}^{a 1} b_{1,1} R_{1} R_{1} \partial \xi \\
\int_{0}^{a 2} b_{2,1} Q_{1} Q_{1} \partial \xi
\end{array}\right\}
$$

Onde:

$$
\begin{aligned}
& Q_{j}\left(\xi_{i} / a_{i}\right)=\left(\frac{\xi_{i}}{a_{i}}\right)^{j-1} \Rightarrow Q_{1}\left(\xi_{1} / a_{1}\right)=\left(\frac{\xi_{1}}{a_{1}}\right)^{1-1}=\left(\frac{\xi_{1}}{a_{1}}\right)^{0}=1 \\
& R_{j}\left(\xi_{i} / a_{i}\right)=\frac{1}{\sqrt{1+\xi_{i}}} \cdot\left(\frac{\xi_{i}}{a_{i}}\right)^{j-1} \quad \text { (base aprox. } \mathrm{p} / \text { fissuras na vizinhança de furos) }
\end{aligned}
$$

Importante ressaltar que o sistema linear dado na equação (5.45) possui duas linhas sendo a primeira representativa da linha de fissura número 1 e a segunda referente à linha de fissura 2. Devido a primeira fissura localizar-se na região do furo optou-se pela utilização da função ponderadora $\boldsymbol{R}_{\boldsymbol{j}}$ (note que na primeira linha do sistema linear a segunda base aproximativa de cada integral é sempre $\boldsymbol{R}_{\boldsymbol{j}}$ ). Em virtude da segunda fissura localizar-se em uma região ausente de furos, optou-se em utilizar a função ponderadora $\boldsymbol{Q}_{j}$ (note que na segunda linha do sistema linear a segunda base aproximativa de cada integral é sempre $\boldsymbol{Q}_{j}$ ). 
No que se refere à influência entre fissuras, observa-se que a integral da linha de fissura-borda que contêm o parâmetro $c_{1,1}^{(2)} Q_{1}$ possui aproximação da tensão por uma base polinomial devido aos carregamentos aproximativos aplicados na fissura 2 serem polinomiais; desta maneira, a influência da fissura 2 sobre a fissura-borda é oriunda de uma base de funções polinomiais. O contrário ocorre na segunda linha do sistema linear: a integral da linha de fissura 2 (borda simples) que contêm o parâmetro $c_{2,1}^{(1)} R_{1}$ possui aproximação da tensão por uma base de funções racionais devido à mesma receber influência dos carregamentos $\boldsymbol{R}_{\boldsymbol{j}}$ aplicados na fissura de borda-furo. Já as integrais pertencentes à diagonal principal da matriz [IG] referem-se às tensões dos subproblemas locais $P_{L}^{(k)}$ que ocorrem nas faces das fissuras. $\mathrm{O}$ primeiro elemento refere-se à fissura-furo e o segundo à fissura de borda simples. Desta maneira, estendendo-se à análise para um número maior de termos aproximativos, obtêm-se os resultados mostrados na tabela 5.14, com precisões similares às obtidas anteriormente.

Tabela 5.14 - Valores de K obtidos para o exemplo contendo

fissura de borda-furo e fissura de borda simples

\begin{tabular}{|c|c|c|c|c|c|}
\hline \multicolumn{6}{|c|}{ Carregamento Constante } \\
\hline \multicolumn{3}{|c|}{ Fissura 1: $K_{\mathrm{REF}}=2,602$} & \multicolumn{3}{|c|}{ Fissura 2: $K_{\mathrm{REF}}=1,800$} \\
\hline $\begin{array}{c}\mathrm{N}^{\circ} \text { termos } \\
\text { Aprox. }\end{array}$ & $\mathbf{K}_{\text {APROX }}$ & $\mathrm{E}(\%)$ & $\begin{array}{c}\mathrm{N}^{0} \text { termos } \\
\text { Aprox. }\end{array}$ & $\mathbf{K}_{\text {APROX }}$ & $\mathrm{E}(\%)$ \\
\hline 1 & 1,769 & 31,98 & 1 & 1,954 & 8,54 \\
\hline 2 & 3,744 & 43,9 & 2 & 1,658 & 7,86 \\
\hline 3 & 2,645 & 1,67 & 3 & 1,774 & 1,46 \\
\hline 5 & 2,603 & 0,03 & 5 & 1,824 & 1,33 \\
\hline
\end{tabular}




\section{Método dos Elementos Finitos Generalizados na Análise do Subproblema Local}

\subsection{Problemas Envolvendo Singularidades e a Estratégia de Enriquecimento}

Como se sabe, em problemas contendo fissuras, os gradientes das tensões no entorno das suas pontas é muito elevado. Quando do emprego do MEF clássico para a análise desse tipo de problema, observa-se a obtenção de resultados insatisfatórios no que diz respeito à representação de grandezas que dependam de ordens superiores de derivadas dos campos de aproximação. Tal fato ocorre devido à natureza polinomial regular dos campos de aproximação empregados, incapazes de reproduzir gradientes localizados elevados, Szabó e Babuška (1991). 
Dentre estas alternativas para a obtenção de uma melhor representatividade dos campos de tensão destacam-se o refinamento da malha de elementos finitos na região do entorno da ponta de fissura, com um conseqüente aumento no custo computacional e a utilização de elementos finitos especiais (quarter-point) que aproximam melhor a singularidade local da solução exata. Esta alternativa produz resultados satisfatórios, porém apresenta uma implementação especial para o elemento finito em questão gerando uma não uniformidade na montagem da matriz de rigidez da estrutura.

O Método dos Elementos Finitos Generalizados (MEFG) proporciona uma alternativa para a obtenção de resultados satisfatórios no que diz respeito à representatividade dos campos de tensão, de menor custo computacional do que aquele apresentado mediante a utilização de malhas excessivamente refinadas. Tal método resulta da combinação do MEF convencional, uma vez que utiliza uma malha de elementos, com conceitos e técnicas típicas dos métodos sem malha, particularmente quanto à adição de funções enriquecedoras (e parâmetros a ela associados) atrelados a pontos nodais do domínio. Outro aspecto importante do MEFG é o fato de explorar o conceito de partição de unidade atrelado às funções de forma do tipo lagrangianas dos elementos, permitindo realizar o enriquecimento local da aproximação com funções (associadas aos nós) apropriadas ao referido caso, sem que haja perda de conformidade entre os elementos.

O presente capítulo trata da aplicação do MEFG na resolução do subproblema local do Método da Partição, possibilitando a obtenção dos fatores de intensidade de tensão e dos campos de tensão com malhas pouco refinadas. Em seguida, são apresentados um breve histórico e aspectos essenciais da formulação do MEFG, assim como os procedimentos necessários para a utilização da solução analítica do problema de fratura como função enriquecedora da aproximação. Posteriormente são apresentados casos clássicos da mecânica da fratura analisados via MEFG exclusivamente, além de casos resolvidos mediante o Método da Partição com enriquecimento do subproblema local pelo MEFG.

\subsection{Breve Histórico Sobre o MEFG}

Vários foram os trabalhos que deram origem ao chamado Método dos Elementos Finitos Generalizados (MEFG). Segundo Duarte, Babuška e Oden (2000) estes trabalhos são: 
Melenk e Babuška (1996), Babuška e Melenk (1997) com o Método dos Elementos Finitos Partição da Unidade (MEFPU): tal método é apresentado como uma forma de obter boas condições de aproximação local e, além disso, assegurar a conformidade da solução global por meio do uso da partição da unidade, situação que não é assegurada pelo MEF clássico;

Duarte e Oden (1995), Duarte e Oden (1996), Duarte (1996) com trabalhos correspondentes à formulação do Método das Nuvens $h p$ : sua principal característica é a possibilidade de enriquecer a aproximação polinomial original, obtida a partir do Método dos Mínimos Quadrados Móveis (MMQM), sem acréscimo no número de pontos nodais, mas adicionando novos parâmetros a estes nós;

Oden, Duarte e Zienkiewicz (1998): apresenta-se aqui a possibilidade do emprego de rede de elementos finitos para a definição dos nós e das nuvens e a realização do enriquecimento (conforme o Método das Nuvens $h p$ ) sobre as funções de forma lagrangianas usadas no MEF convencional.

Porém, seu emprego sob o nome de Método dos Elementos Finitos Generalizados surgiu pela primeira vez em Melenk (1995), sendo sua fundamentação mais precisamente apresentada em Strouboulis, Babuška e Cops (2000). A seguir são definidas as características principais do MEFG:

Características oriundas do MEF:

$\checkmark$ Simplicidade na geração da partição de unidade devido à utilização da interpolação lagrangiana;

$\checkmark$ A malha de elementos finitos é a mesma para a integração numérica;

$\checkmark$ A questão do MEF ser um método bastante testado;

$\checkmark$ O fato de existir uma gama enorme de códigos para o MEF que podem ser utilizados para a implementação do MEFG e

$\checkmark$ Facilidade na imposição das condições de contorno.

Características oriundas dos métodos sem malha: 
$\checkmark$ Realizar um enriquecimento optando-se por funções que melhor representem o comportamento da solução exata do problema, possibilitando uma melhor taxa de convergência da resposta;

$\checkmark$ Maior facilidade de realizar o refinamento $\mathbf{p}$, acrescentando novos parâmetros aos nós já existentes e

$\checkmark$ Possibilidade de enriquecer a aproximação em apenas uma região delimitada do domínio global sem comprometimento da conformidade entre os elementos.

Desta maneira, a aplicação do MEFG torna-se bastante interessante nos problemas de fratura devido à possibilidade da utilização de funções enriquecedoras que simulam o efeito de concentração de tensão em um dado elemento, sem a necessidade de alteração na malha. Sendo assim, torna-se possível a utilização da técnica de enriquecimento da aproximação em uma região limitada no entorno da fissura com funções que representam a solução analítica do problema. Tal procedimento dispensa uma nova geração de malha e um ganho substancial no que concerne à rapidez da análise e qualidade de resposta se comparado à aproximação com bases polinomiais oriundas do MEF.

\subsection{A Formulação do Método dos Elementos Finitos Generalizados}

Tendo em vista que o MEFG possui características do Método das Nuvens $h p$, ressalta-se o conceito de nuvem ou região de influência $\left(\omega_{j}\right)$ de um dado nó. No caso, dada uma malha de elementos finitos, as nuvens nodais são definidas pelos conjuntos de elementos que dividem um nó comum. A figura 6.1 ilustra este conceito com nuvens atreladas aos nós A e B de uma malha regular de elementos finitos.

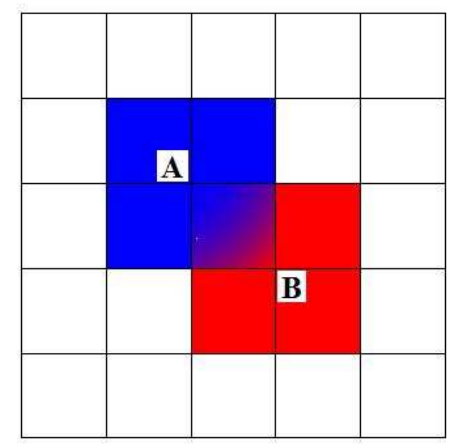

Região de influência do nó $\mathrm{A}$

Região de influência do nó $B$

Região de influência dos nós A e B

Figura 6.1 - Nuvens de influência dos nós A e B de uma malha regular de elementos finitos quadrilaterais 
Outro conceito importante é o de Partição de Unidade (PU). A Partição da Unidade é obtida mediante a combinação das funções de forma dos elementos finitos que compõem uma nuvem e atrelados ao nó vértice da mesma. Mais formalmente, o conjunto de funções de forma $\phi_{j}(x)$ definidas nas nuvens $\omega_{j}$ que contêm o ponto $\boldsymbol{x}$, constitui uma PU caso satisfaçam as seguintes condições:

$\checkmark$ As funções de forma pertencem ao conjunto de funções para os quais $\omega_{j}$ é um suporte compacto, ou seja, $\phi_{j}(x)$ e suas derivadas assumem valores não-nulos somente no interior da região $\omega_{j}$;

$\checkmark$ A somatória das funções de forma em qualquer ponto deve ser igual à unidade, ou seja, $\sum_{j=1}^{N} \phi_{j}(x)=1$

$\checkmark$ O valor de cada função de forma em qualquer ponto do domínio $\Omega$ da estrutura deve ser maior ou igual à zero, ou seja, $\phi_{j}(x) \geq 0$ em $\Omega$ e

$\checkmark$ Todo subconjunto compacto de $\Omega$ intercepta apenas um número finito de suportes $\phi_{j}(x)$

O enriquecimento é realizado em cada região de influencia (nuvem) mediante a multiplicação da PU associada a cada nó base por uma função de interesse (possuindo características de boa representatividade da função solução). Com isto, o conjunto das funções de forma associadas aos nós de uma estrutura analisada via MEFG pode ser definida como sendo:

$\mathfrak{I}_{n}^{p}=\left\{\phi_{j} \cup \phi_{j} f_{j}^{\alpha} \quad, \quad j=1, \ldots, n ; \alpha=1, \ldots, F(j)\right\}$ 
Onde:

$n \quad$ - número total de nós da discretização,

$p \quad$ - grau polinomial da família de funções,

$\phi_{j}(x)$ - funções de forma iniciais referentes aos nós $j=1, \ldots, n$,

$f_{j}^{\alpha} \quad$ - função de índice $\alpha$ que multiplica (ou enriquece) a função de forma atrelada a cada nó de índice $j$,

$F(j) \quad$ - contador para o número de funções adicionadas a cada nó de índice $j$.

$\mathrm{Na}$ ilustração da figura 6.2 , definidas as funções enriquecedoras, observa-se que a região de influência (nuvem ou suporte $\omega_{j}$ ) é definida pelo conjunto dos quatro elementos finitos quadrilaterais que possuem vértice comum ao nó $x_{j}$. Nota-se também que a função produto, referente à multiplicação da PU pela função enriquecedora (aproximação local), preserva o suporte compacto da PU, juntamente com as características oriundas da função enriquecedora.

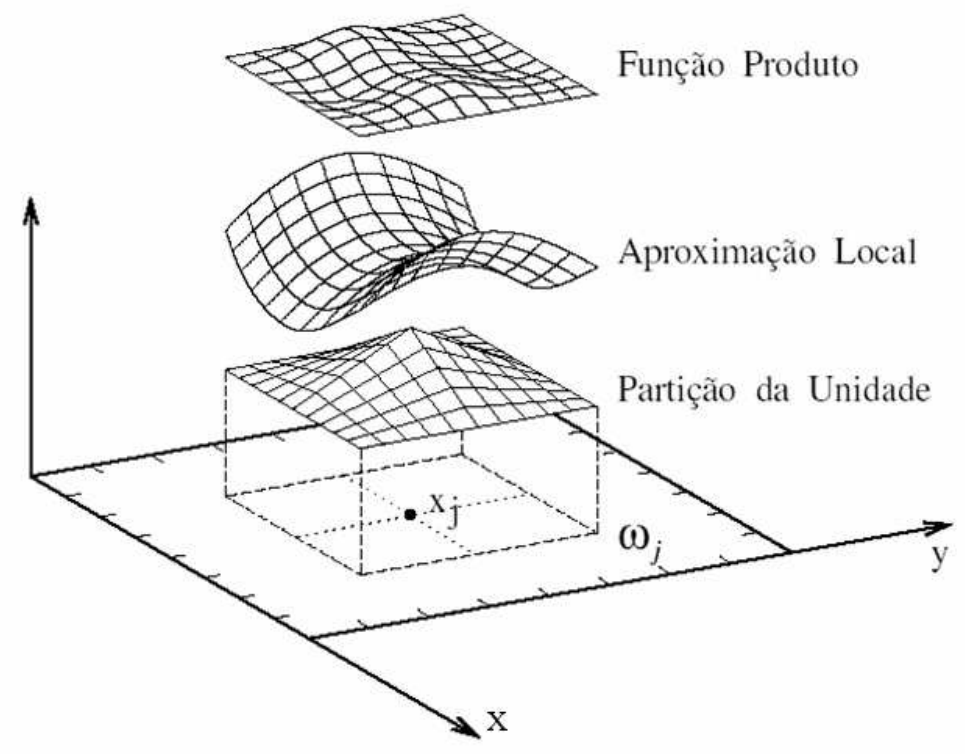

Figura 6.2 - Enriquecimento da partição da Unidade num domínio global bidimensional. 


\subsection{O Elemento Finito Isoparamétrico Enriquecido}

Nas análises numéricas utiliza-se o elemento finito quadrilateral isoparamétrico de quatro nós, sendo que o mesmo possui dois graus de liberdade por nó (deslocamentos segundo direções ortogonais entre si). A transformação de coordenadas do elemento do domínio paramétrico para o domínio físico, representado na figura 6.3 , é realizada mediante a utilização de funções lagrangianas bilineares $\xi$ e $\eta$ (coordenadas adimensionais variando entre -1 e +1), Assan (2003).
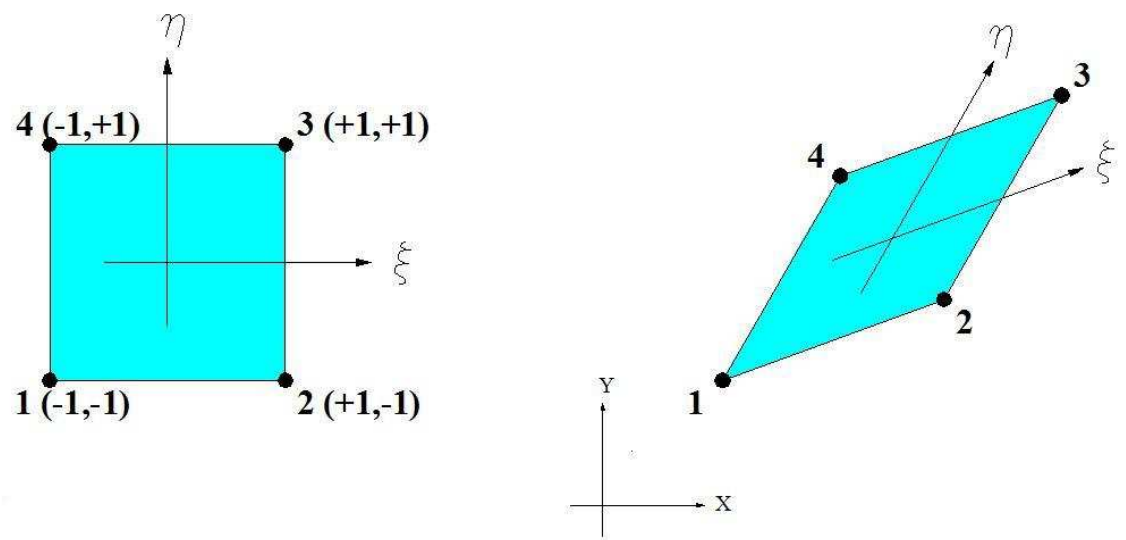

Figura 6.3 - Elemento Finito Quadrilateral de 4 nós

Regular no domínio paramétrico e Distorcido no domínio físico

As funções lagrangianas são as seguintes:

$$
\left\{\begin{aligned}
\phi_{1} & =\frac{1}{4}(1-\xi)(1-\eta) \\
\phi_{2} & =\frac{1}{4}(1+\xi)(1-\eta) \\
\phi_{3} & =\frac{1}{4}(1+\xi)(1+\eta) \\
\phi_{4} & =\frac{1}{4}(1-\xi)(1+\eta)
\end{aligned}\right.
$$

As componentes cartesianas dos deslocamentos nodais do elemento ficam expressas nas formas: 
$\left\{\begin{aligned} u & =\sum_{i=1}^{4} u_{i} \phi_{i} \\ v & =\sum_{i=1}^{4} v_{i} \phi_{i}\end{aligned}\right.$

Onde $\left(u_{i}, v_{i}\right)$ são os deslocamentos nodais do elemento finito isoparamétrico.

$\mathrm{Na}$ estratégia de enriquecimento para cada função enriquecedora adiciona-se um novo grau de liberdade nodal. Por exemplo, caso o nó de número 1 de um dado elemento seja enriquecido na direção $u$ com " $n f u l$ " funções e na direção $v$ com " $n f v l$ ” funções, a equação (6.3) para este mesmo elemento torna-se:

$$
\left\{\begin{array}{c}
u=\left(u_{1}+\sum_{i=1}^{n f u 1} p u_{1}^{i} \cdot f_{u 1}^{i}\right) \phi_{1}+\sum_{i=2}^{4} u_{i} \phi_{i} \\
v=\left(v_{1}+\sum_{i=1}^{n f v 1} p v_{1}^{i} \cdot f_{v 1}^{i}\right) \phi_{1}+\sum_{i=2}^{4} u_{i} \phi_{i}
\end{array}\right.
$$

Detalhes sobre sub-rotinas de métodos numéricos tais como o MEF na análise de problemas da Mecânica da Fratura, assim como procedimentos mais completos abordando a montagem da matriz de rigidez global enriquecida são encontrados, respectivamente, em Owen e Fawkes (1983) e Barros (2002), Torres (2003).

A representação matricial da equação (6.4) é dada da seguinte forma:

$$
\{d\}=[\phi] \cdot\{\delta\}
$$

Onde:

$$
\begin{aligned}
& \{d\}=\left\{\begin{array}{l}
u \\
v
\end{array}\right\} \\
& {[\phi]=\left[\begin{array}{ccccccccccccccccc}
\phi_{1} & \phi_{1} f_{u 1}^{1} & \cdots & \phi_{1} f_{u 1}^{n f u 1} & 0 & 0 & \cdots & 0 & \phi_{2} & 0 & \phi_{3} & 0 & \phi_{4} & 0 \\
0 & 0 & \cdots & 0 & \phi_{1} & \phi_{1} f_{v 1}^{1} & \cdots & \phi_{1} f_{v 1}^{n f v 1} & 0 & \phi_{2} & 0 & \phi_{3} & 0 & \phi_{4}
\end{array}\right]}
\end{aligned}
$$




$$
\{\boldsymbol{\delta}\}=\left\{\begin{array}{c}
u_{1} \\
p u_{1}^{1} \\
\vdots \\
p u_{1}^{n f u 1} \\
v_{1} \\
p v_{1}^{1} \\
\vdots \\
p v_{1}^{n f v 1} \\
u_{2} \\
v_{2} \\
u_{3} \\
v_{3} \\
u_{4} \\
v_{4}
\end{array}\right\}
$$

Por outro lado, a matriz de rigidez $[\mathrm{K}]$ do elemento é dada da seguinte forma:

$$
[\mathrm{K}]=\int_{A} t \cdot[B]^{T} \cdot[D] \cdot[B] d A
$$

A dedução de $[\mathrm{K}]$ depende da obtenção da matriz [B]. A espessura do elemento é dada por $t$ e a matriz [D] apresenta a seguinte forma:

$$
[D]=\frac{E^{\prime}}{\left(1-v^{\prime 2}\right)}\left[\begin{array}{ccc}
1 & v & 0 \\
v & 1 & 0 \\
0 & 0 & \left(\frac{1-v^{\prime}}{2}\right)
\end{array}\right]
$$

onde para o estado plano de tensão têm-se que:

$$
\left\{\begin{array}{l}
E^{\prime}=E \\
v^{\prime}=v
\end{array}\right.
$$


e para o estado plano de deformação têm-se:

$$
\left\{\begin{array}{l}
E^{\prime}=\frac{E}{1-v^{2}} \\
v^{\prime}=\frac{v}{1-v}
\end{array}\right.
$$

Uma vez definida a matriz [D], busca-se então a matriz [B], que relaciona deformações e deslocamentos. Partindo das equações expressas por (6.13) têm-se que:

$$
\left\{\begin{array}{rl}
\varepsilon_{x} & =\frac{\partial u}{\partial x} \\
\varepsilon_{y} & =\frac{\partial v}{\partial y} \\
\gamma_{x y} & =\frac{\partial u}{\partial y}+\frac{\partial v}{\partial x}
\end{array} \Rightarrow\left\{\begin{array}{c}
\varepsilon_{x} \\
\varepsilon_{y} \\
\gamma_{x y}
\end{array}\right\}=\left[\begin{array}{llll}
1 & 0 & 0 & 0 \\
0 & 0 & 0 & 1 \\
0 & 1 & 1 & 0
\end{array}\right]\left\{\begin{array}{l}
\partial u / \partial x \\
\partial u / \partial y \\
\partial v / \partial x \\
\partial v / \partial y
\end{array}\right\} \Rightarrow\{\varepsilon\}=[I]\{\partial x y\}\right.
$$

A matriz [B] (que relaciona deslocamentos e deformações) fica expressa a partir de uma nova relação da equação 6.13 , deixando-se em evidência o vetor deslocamento $\{\delta\}^{T}=\left\{\begin{array}{ll}u & v\end{array}\right\}$ :

$$
\{\varepsilon\}=[I]\{\partial x y\} \Rightarrow \underbrace{\{\varepsilon\}}_{\text {deformações }}=\underbrace{[I][\ldots]}_{\text {matriz B }} \cdot \underbrace{\{\delta\}}_{\text {deslocamentos }}
$$

Como na equação 6.13 os deslocamentos do elemento finito isoparamétrico estão em função das coordenadas paramétricas $\xi$ e $\eta$, as derivadas do vetor $\{\partial x y\}$ são dadas por:

$$
\left\{\begin{array}{l}
\frac{\partial u}{\partial x}=\frac{\partial u}{\partial \xi} \frac{\partial \xi}{\partial x}+\frac{\partial u}{\partial \eta} \frac{\partial \eta}{\partial x} \\
\frac{\partial u}{\partial y}=\frac{\partial u}{\partial \xi} \frac{\partial \xi}{\partial y}+\frac{\partial u}{\partial \eta} \frac{\partial \eta}{\partial y} \\
\frac{\partial v}{\partial x}=\frac{\partial v}{\partial \xi} \frac{\partial \xi}{\partial x}+\frac{\partial v}{\partial \eta} \frac{\partial \eta}{\partial x} \\
\frac{\partial v}{\partial y}=\frac{\partial v}{\partial \xi} \frac{\partial \xi}{\partial y}+\frac{\partial v}{\partial \eta} \frac{\partial \eta}{\partial y}
\end{array} \quad \Rightarrow\left\{\begin{array}{l}
\frac{\partial u}{\partial x} \\
\frac{\partial u}{\partial y} \\
\frac{\partial v}{\partial x} \\
\frac{\partial v}{\partial y}
\end{array}\right\}=\left[\begin{array}{cccc}
\frac{\partial \xi}{\partial x} & \frac{\partial \eta}{\partial x} & 0 & 0 \\
\frac{\partial \xi}{\partial y} & \frac{\partial \eta}{\partial y} & 0 & 0 \\
0 & 0 & \frac{\partial \xi}{\partial x} & \frac{\partial \eta}{\partial x} \\
0 & 0 & \frac{\partial \xi}{\partial y} & \frac{\partial \eta}{\partial y}
\end{array}\right]\left\{\begin{array}{l}
\frac{\partial u}{\partial \xi} \\
\frac{\partial u}{\partial \eta} \\
\frac{\partial v}{\partial \xi} \\
\frac{\partial v}{\partial \eta}
\end{array}\right\}\right.
$$


A equação (6.15) pode ser representada da seguinte forma matricial:

$$
\{\partial x y\}=\left[\begin{array}{cc}
{[H]} & {[0]} \\
{[0]} & {[H]}
\end{array}\right]\{\partial \xi \eta\}
$$

onde [0] indica uma submatriz $2 \times 2$ nula e [H] é uma submatriz $2 \times 2$, inversa da submatriz denominada "Jacobiano" [J]:

$[J]=\left[\begin{array}{ll}\frac{\partial x}{\partial \xi} & \frac{\partial y}{\partial \xi} \\ \frac{\partial x}{\partial \eta} & \frac{\partial y}{\partial \eta}\end{array}\right]$

$[H]=[J]^{-1}=\frac{1}{\operatorname{det}[J]}\left[\begin{array}{cc}J_{22} & -J_{12} \\ -J_{21} & J_{11}\end{array}\right]=\frac{1}{\frac{d x}{d \xi} \frac{d y}{d \eta}-\frac{d y}{d \xi} \frac{d x}{d \eta}}\left[\begin{array}{cc}\frac{d y}{d \eta} & -\frac{d y}{d \xi} \\ -\frac{d x}{d \eta} & \frac{d x}{d \xi}\end{array}\right]$

As derivadas das coordenadas globais $x$ e $y$ em relação às coordenadas paramétricas $\xi$ e $\eta$ podem ser obtidas facilmente, e por conseqüência as submatrizes $[H]$. Já para o desenvolvimento do vetor $\{\partial \xi \eta\}$, que possui as derivadas dos valores de $u$ e $v$ em relação à $\xi$ e $\eta$, parte-se do sistema linear $\{d\}=[\phi] \cdot\{\delta\}$ (equação (6.5)), ou seja:

$$
\begin{aligned}
& u=u_{1} \phi_{1}+p u_{1}^{1} \phi_{1} f_{u 1}^{1}+\cdots+p u_{1}^{n f u 1} \phi_{1} f_{u 1}^{n f u 1}+u_{2} \phi_{2}+u_{3} \phi_{3}+u_{4} \phi_{4} \\
& v=v_{1} \phi_{1}+p v_{1}^{1} \phi_{1} f_{v 1}^{1}+\cdots+p v_{1}^{n f v 1} \phi_{1} f_{v 1}^{n f v 1}+v_{2} \phi_{2}+v_{3} \phi_{3}+v_{4} \phi_{4}
\end{aligned}
$$

Derivando as equações (6.19) e (6.20) em relação às coordenadas paramétricas $\xi$ e $\eta$ e expressando estas quatro equações na forma matricial têm-se que: 
$\left\{\begin{array}{l}\frac{\partial u}{\partial \xi} \\ \frac{\partial u}{\partial \eta} \\ \frac{\partial v}{\partial \xi} \\ \frac{\partial v}{\partial \eta}\end{array}\right\}=\left[\begin{array}{ccccc}\frac{\partial \phi_{1}}{\partial \xi} & \left(\frac{\partial \phi_{1}}{\partial \xi} f_{u 1}^{1}+\frac{\partial f_{u 1}^{1}}{\partial \xi} \phi_{1}\right) & \ldots & \left(\frac{\partial \phi_{1}}{\partial \xi} f_{u 1}^{n f u 1}+\frac{\partial f_{u 1}^{n f u 1}}{\partial \xi} \phi_{1}\right) & \ldots \\ \frac{\partial \phi_{1}}{\partial \eta} & \left(\frac{\partial \phi_{1}}{\partial \eta} f_{u 1}^{1}+\frac{\partial f_{u 1}^{1}}{\partial \eta} \phi_{1}\right) & \ldots & \left(\frac{\partial \phi_{1}}{\partial \eta} f_{u 1}^{n f u 1}+\frac{\partial f_{u 1}^{n f u 1}}{\partial \eta} \phi_{1}\right) & \ldots \\ 0 & 0 & \ldots & 0 & \ldots \\ 0 & 0 & \ldots & 0 & \ldots\end{array}\right.$

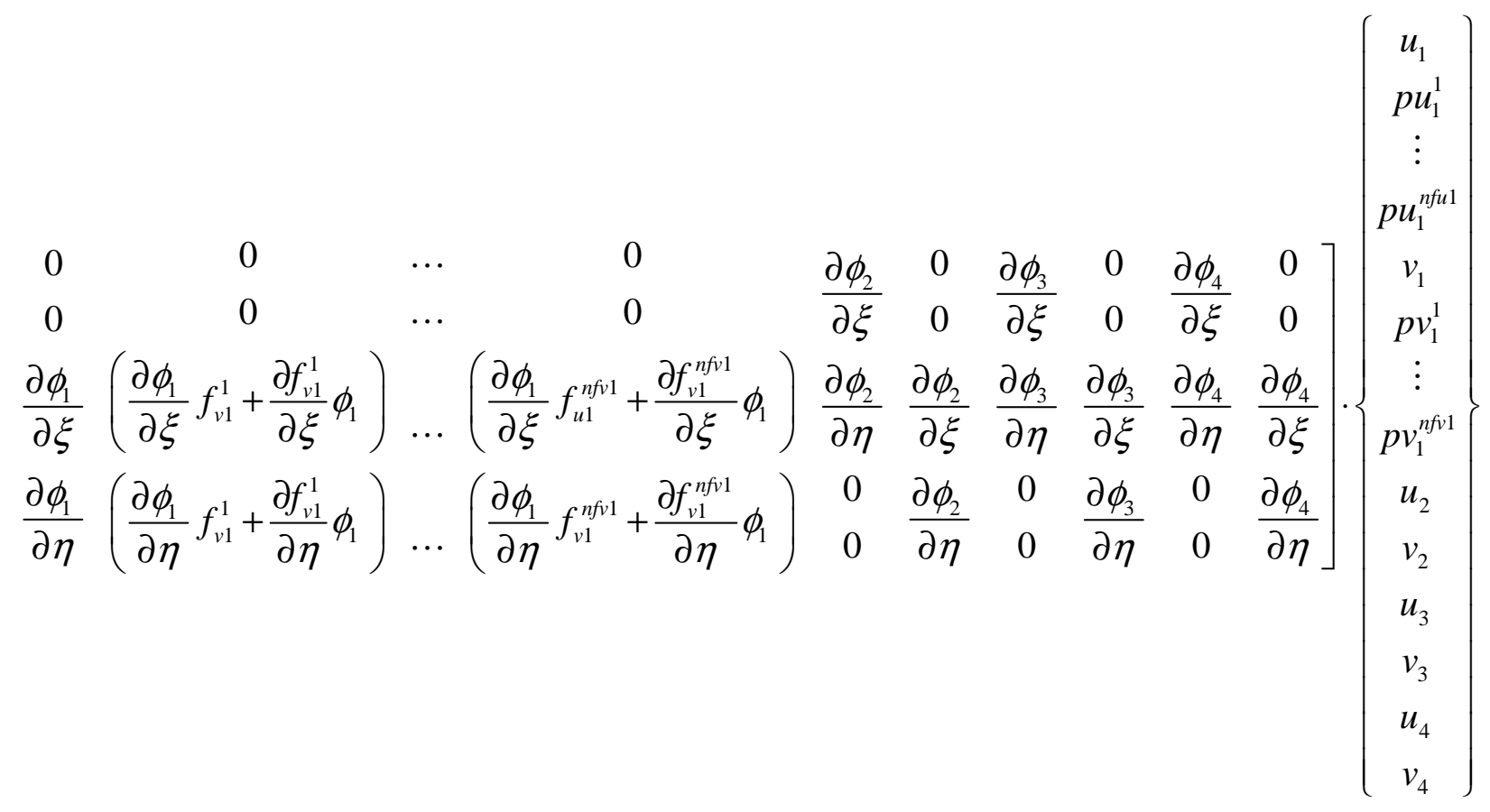

A representação matricial da equação (6.21) é dada da seguinte forma:

$\{\partial \xi \eta\}=[\partial \phi f] \cdot\{\delta\}$

Inserindo a equação (6.22) em (6.16) e posteriormente o resultado disto na equação (6.13) obtêm-se:

$$
\begin{aligned}
& \{\partial x y\}=\left[\begin{array}{cc}
{[H]} & {[0]} \\
{[0]} & {[H]}
\end{array}\right]\{\partial \xi \eta\}=\left[\begin{array}{cc}
{[H]} & {[0]} \\
{[0]} & {[H]}
\end{array}\right] \cdot[\partial \phi f]\{\delta\} \\
& \{\varepsilon\}=[I]\{\partial x y\}=[I] \cdot\left[\begin{array}{cc}
{[H]} & {[0]} \\
{[0]} & {[H]}
\end{array}\right][\partial \phi f]\{\delta\}
\end{aligned}
$$


Observando a equação (6.23), nota-se que o vetor de deformações $\{\varepsilon\}$ tem explicitado o vetor de deslocamentos $\{\delta\}$. Como a matriz [B] relaciona deformações com deslocamentos, define-se, então, a matriz [B] pela multiplicação das seguintes matrizes:

$$
\{\varepsilon\}=\underbrace{[I] \cdot\left[\begin{array}{cc}
{[H]} & {[0]} \\
{[0]} & {[H]}
\end{array}\right][\partial \phi f]}_{\text {matriz } B}\{\delta\}=[B] \cdot\{\delta\}
$$

O vetor de forças nodais $\{F\}$ "para forças aplicadas diretamente nos nós" é dado da seguinte maneira:

$$
\{F\}=\left\{\begin{array}{c}
F_{u 1} \\
F_{u 1} \cdot f_{u 1}^{1} \\
\vdots \\
F_{u 1} \cdot f_{u 1}^{n f u 1} \\
F_{v 1} \\
F_{v 1} \cdot f_{v 1}^{1} \\
\vdots \\
F_{v 1} \cdot f_{v 1}^{n f v 1} \\
F_{u 2} \\
F_{v 2} \\
F_{u 3} \\
F_{v 3} \\
F_{u 4} \\
F_{v 4}
\end{array}\right\}
$$

onde $F_{i j}$ representa a força nodal aplicada na direção $i$ do nó $\boldsymbol{j}$ do elemento finito em questão. Sendo $f_{i, j}^{n}$ o valor da função enriquecedora número $\boldsymbol{n}$ aplicada na direção $\boldsymbol{i}$ no nó $\boldsymbol{j}$ 


\subsection{A Solução Analítica da Fratura como Função Enriquecedora do Subproblema Local do Método da Partição}

Szabó e Babuška (1991) apresentam na forma de uma série infinita, a seguinte solução analítica do campo bidimensional de deslocamentos para problemas da Mecânica da Fratura:

$$
\begin{aligned}
& u_{x^{\prime}}(r, \theta)=\sum_{\alpha=1}^{\infty}\left[A_{\alpha}^{(1) \alpha} u_{x^{\prime}}^{(1)}+A_{\alpha}^{(2) \alpha} u_{x^{\prime}}^{(2)}\right]+\tilde{u}_{x^{\prime}}(r, \theta) \\
& u_{y^{\prime}}(r, \theta)=\sum_{\alpha=1}^{\infty}\left[A_{\alpha}^{(1) \alpha} u_{y^{\prime}}^{(1)}+A_{\alpha}^{(2) \alpha} u_{y^{\prime}}^{(2)}\right]+\tilde{u}_{y^{\prime}}(r, \theta)
\end{aligned}
$$

onde os índices (1) e (2) representam os modos I e II de abertura, respectivamente, $r$ e $\theta$ representam as coordenadas polares que possuem origem na ponta da fissura $(r=0)$. As parcelas $\tilde{u}_{x^{\prime}}(r, \theta)$ e $\tilde{u}_{y^{\prime}}(r, \theta)$ são funções mais suaves do que os demais termos do somatório da série infinita.

Por simplificação, considera-se somente o primeiro termo da série infinita nas equações (6.26) e (6.27), sendo as mesmas podendo ser reescritas da seguinte forma:

$$
\begin{aligned}
& u_{x^{\prime}}(r, \theta)=A_{1}^{(1)} u_{x^{\prime}}^{(1)}+A_{1}^{(2)} u_{x^{\prime}}^{(2)} \\
& u_{y^{\prime}}(r, \theta)=A_{1}^{(1)} u_{y^{\prime}}^{(1)}+A_{1}^{(2)} u_{y^{\prime}}^{(2)}
\end{aligned}
$$

Os termos $A_{1}^{(1)}$ e $A_{1}^{(2)}$ são:

$$
\begin{aligned}
& A_{1}^{(1)}=\frac{K_{I}}{\sqrt{2 \pi}} \\
& A_{1}^{(2)}=\frac{K_{I I}}{\sqrt{2 \pi}}
\end{aligned}
$$

e as auto-funções $u_{x^{\prime}}^{(1)}, u_{x^{\prime}}^{(2)}, u_{y^{\prime}}^{(1)}, u_{y^{\prime}}^{(2)}$ para o primeiro termo da série são dadas por: 


$$
\begin{aligned}
& u_{x^{\prime}}^{(1)}=\frac{r^{\lambda_{1}}}{2 G}\left\{\left[k-Q_{1}^{(1)}\left(\lambda_{1}+1\right)\right] \cos \left(\lambda_{1} \theta\right)-\lambda_{1} \cos \left(\left(\lambda_{1}-2\right) \theta\right)\right\} \\
& u_{y^{\prime}}^{(1)}=\frac{r^{\lambda_{1}}}{2 G}\left\{\left[k+Q_{1}^{(1)}\left(\lambda_{1}+1\right)\right] \operatorname{sen}\left(\lambda_{1} \theta\right)+\lambda_{1} \operatorname{sen}\left(\left(\lambda_{1}-2\right) \theta\right)\right\} \\
& u_{x^{\prime}}^{(2)}=\frac{r^{\lambda_{1}}}{2 G}\left\{\left[k-Q_{1}^{(2)}\left(\lambda_{1}+1\right)\right] \operatorname{sen}\left(\lambda_{1} \theta\right)-\lambda_{1} \operatorname{sen}\left(\left(\lambda_{1}-2\right) \theta\right)\right\} \\
& u_{y^{\prime}}^{(2)}=\frac{-r^{\lambda_{1}}}{2 G}\left\{\left[k+Q_{1}^{(2)}\left(\lambda_{1}+1\right)\right] \cos \left(\lambda_{1} \theta\right)+\lambda_{1} \cos \left(\left(\lambda_{1}-2\right) \theta\right)\right\}
\end{aligned}
$$

Assim como nas equações (6.26) e (6.27), os expoentes (1) e (2) das equações (6.32) a (6.35) representam os modos I e II de abertura, respectivamente. Já os índices unitários de $Q_{1}^{(1)}, Q_{1}^{(2)}$ e $\lambda_{1}$ representam os valores destas variáveis para o termo número 1 da série; caso fosse considerado um outro termo da série, estas três variáveis assumiriam outros valores. No caso de apenas um termo da série, estas variáveis assumem os seguintes valores:

Modo I de abertura:

$$
\left\{\begin{array}{l}
\lambda_{1}=\frac{1}{2} \\
Q_{1}^{(1)}=\frac{1}{3}
\end{array}\right.
$$

Modo II de abertura:

$$
\left\{\begin{array}{l}
\lambda_{1}=\frac{1}{2} \\
Q_{1}^{(2)}=-1
\end{array}\right.
$$

Dentre outros parâmetros que aparecem nas equações (6.32) a (6.35) destacam-se:

$$
k=\left\{\begin{array}{lll}
3-4 v & \text { se } & E P D \\
\frac{3-v}{1+v} & \text { se } & E P T
\end{array}\right.
$$


$G=\frac{E}{2(1+v)}$

$\theta=\theta_{G}-\theta_{0}$

Conforme ilustra a figura 6.4 , o ângulo $\boldsymbol{\theta}$, presente nas equações (6.32) a (6.35), é a diferença entre os ângulos $\boldsymbol{\theta}_{\boldsymbol{G}}$ e $\boldsymbol{\theta}_{0}$. Sendo o ângulo $\boldsymbol{\theta}_{\boldsymbol{G}}$ a inclinação da semi-reta de comprimento $\boldsymbol{r}$ e que possui seus limites dados pelos pontos $\mathrm{O}$ e $\mathrm{P}$, respectivamente a origem na ponta da fissura e o ponto de integração numérica, por exemplo. Este segmento $\overline{\boldsymbol{O P}}$, ou seja, $\boldsymbol{r}$, tem sua inclinação medida com base em um eixo horizontal $\mathbf{X}$ de referência global, conforme ilustra a figura 6.4 . Já o ângulo $\boldsymbol{\theta}_{0}$ refere-se à inclinação da fissura, que é medida entre o segmento de reta que possui origem na raiz da fissura (eixo $\mathbf{X}^{\prime}$ ) e o mesmo eixo horizontal $\mathbf{X}$ descrito anteriormente. Os ângulos $\boldsymbol{\theta}_{\boldsymbol{G}}$ e $\boldsymbol{\theta}_{0}$ variam entre 0 e $180^{\circ}$, iniciando com valores nulos a partir do eixo horizontal $\mathbf{X}$, positivos se o segmento $\overline{\boldsymbol{O P}}$ ou eixo $\mathbf{X}^{\prime}$ forem obtidos mediante uma rotação no sentido anti-horário, e negativos se o segmento $\overline{\boldsymbol{O P}}$ ou eixo $\mathbf{X}^{\prime}$ forem obtidos mediante uma rotação no sentido horário. Na figura 6.4 , os três ângulos mostrados possuem valores positivos.

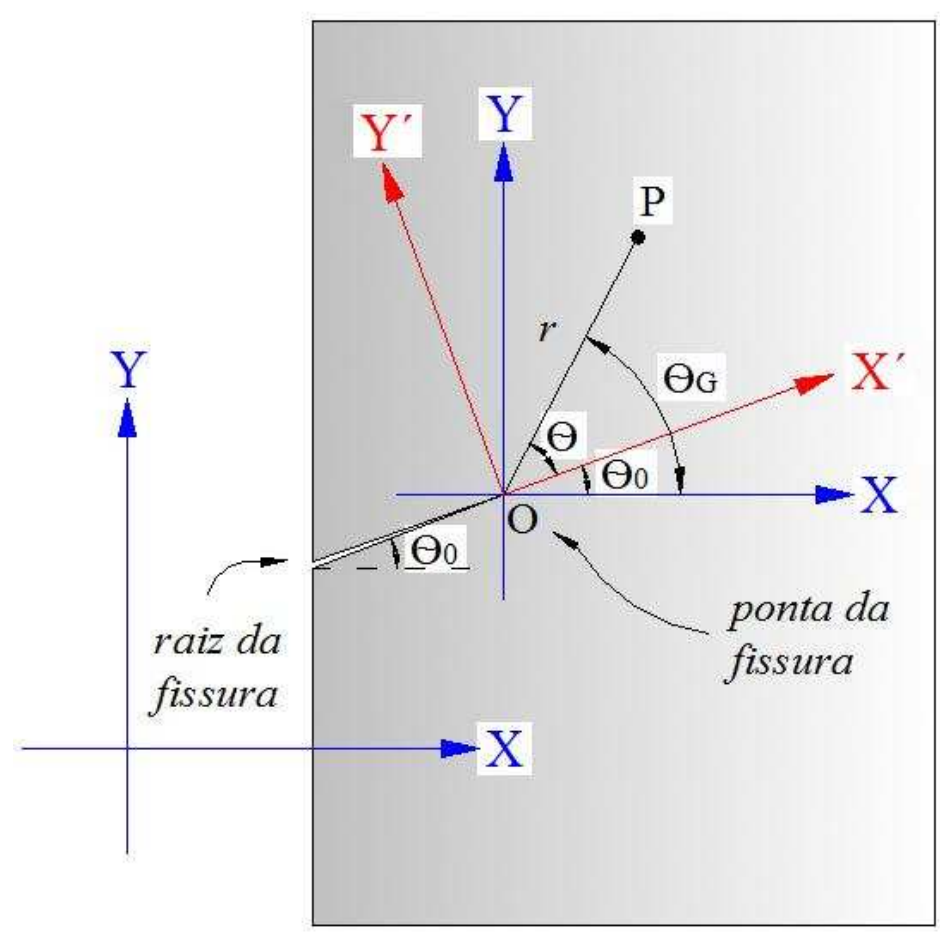

Figura 6.4 - Orientação dos eixos locais da fissura 
Uma vez definidas as equações de deslocamentos (6.32) a (6.35) observa-se pela matriz da equação (6.21) que faz-se necessário a definição das funções enriquecedoras em deslocamentos para a determinação da matriz [B], assim como as derivadas destas funções em relação às coordenadas paramétricas $\xi$ e $\eta$.

No caso de "fissuras horizontais", ou seja, quando os eixos $\mathbf{X}^{\prime} \mathbf{Y}^{\prime}$ coincidem com os eixos globais $\mathbf{X Y}$ as funções enriquecedoras em deslocamentos para o modo I de abertura ficam definidas como sendo:

$$
\begin{aligned}
& u_{x}^{(1)}=\frac{r^{\lambda_{1}}}{2 G}\left\{\left[k-Q_{1}^{(1)}\left(\lambda_{1}+1\right)\right] \cos \left(\lambda_{1} \theta\right)-\lambda_{1} \cos \left(\left(\lambda_{1}-2\right) \theta\right)\right\} \\
& u_{y}^{(1)}=\frac{r^{\lambda_{1}}}{2 G}\left\{\left[k+Q_{1}^{(1)}\left(\lambda_{1}+1\right)\right] \operatorname{sen}\left(\lambda_{1} \theta\right)+\lambda_{1} \operatorname{sen}\left(\left(\lambda_{1}-2\right) \theta\right)\right\}
\end{aligned}
$$

e para o modo II de abertura são:

$$
\begin{aligned}
& u_{x}^{(2)}=\frac{r^{\lambda_{1}}}{2 G}\left\{\left[k-Q_{1}^{(2)}\left(\lambda_{1}+1\right)\right] \operatorname{sen}\left(\lambda_{1} \theta\right)-\lambda_{1} \operatorname{sen}\left(\left(\lambda_{1}-2\right) \theta\right)\right\} \\
& u_{y}^{(2)}=\frac{-r^{\lambda_{1}}}{2 G}\left\{\left[k+Q_{1}^{(2)}\left(\lambda_{1}+1\right)\right] \cos \left(\lambda_{1} \theta\right)+\lambda_{1} \cos \left(\left(\lambda_{1}-2\right) \theta\right)\right\}
\end{aligned}
$$

No caso de "fissuras inclinadas" as funções enriquecedoras expressas por (6.32) a (6.35) e que possuem sua orientação em relação em relação aos eixos $X^{\prime} Y^{\prime}$ devem ser transformadas segundo os eixos globais $\mathrm{X} \mathrm{e} \mathrm{Y}$, devido ao enriquecimento ser realizado em relação às variáveis globais. No caso, as funções enriquecedoras para o modo I de abertura são dadas por:

$$
\begin{aligned}
& u_{x}^{(1)}=u_{x^{\prime}}^{(1)} \cos \theta_{0}-u_{y^{\prime}}^{(1)} \operatorname{sen} \theta_{0} \\
& u_{y}^{(1)}=u_{x^{\prime}}^{(1)} \operatorname{sen} \theta_{0}+u_{y^{\prime}}^{(1)} \cos \theta_{0}
\end{aligned}
$$

e para o modo II de abertura estas funções são expressas por:

$$
\begin{aligned}
& u_{x}^{(2)}=u_{x^{\prime}}^{(2)} \cos \theta_{0}-u_{y^{\prime}}^{(2)} \operatorname{sen} \theta_{0} \\
& u_{y}^{(2)}=u_{x^{\prime}}^{(2)} \operatorname{sen} \theta_{0}+u_{y^{\prime}}^{(2)} \cos \theta_{0}
\end{aligned}
$$


Uma vez definidas as funções enriquecedoras em deslocamentos, basta então a obtenção de suas derivadas em relação às coordenadas paramétricas $\xi$ e $\eta$ para a montagem da matriz [B]. Para isso, emprega-se a regra da cadeia, em virtude das variáveis $u_{x}^{(1)}, u_{x}^{(2)}$, $u_{y}^{(1)}, u_{y}^{(2)}$ estarem em função das projeções de $u_{x^{\prime}}^{(1)}, u_{x^{\prime}}^{(2)}, u_{y^{\prime}}^{(1)}, u_{y^{\prime}}^{(2)}$ sobre o eixo $\mathbf{X Y}$, das coordenadas polares $\boldsymbol{r}$ e $\boldsymbol{\theta}$, das coordenadas dos eixos $\mathbf{X}^{\prime} \mathbf{Y}^{\prime}$ e $\mathbf{X Y}$ e conseqüentemente das coordenadas paramétricas $\xi$ e $\eta$. Esta idéia está indicada na seqüência seguinte:

$$
\begin{aligned}
& u_{x}^{(\ldots)}, u_{x}^{(\ldots)}=f\left(u_{x^{\prime}}^{(\ldots)}, u_{x^{\prime}}^{(\ldots)}\right) \quad \Rightarrow \\
& u_{x^{\prime}}^{(\ldots)}, u_{x^{\prime}}^{(\ldots)}=f(r, \theta) \Rightarrow \\
& r, \theta=f\left(\mathrm{X}^{\prime}, \mathrm{Y}^{\prime}\right) \Rightarrow \\
& \mathrm{X}^{\prime}, \mathrm{Y}^{\prime}=f(\mathrm{X}, \mathrm{Y}) \quad \Rightarrow \\
& \mathrm{X}, \mathrm{Y}=f(\xi, \eta)
\end{aligned}
$$

Tendo conhecimento das dependências entre as variáveis envolvidas e tomando-se a equação (6.44), por exemplo, realiza-se então, para ilustração, a sua derivada em relação à variável paramétrica $\xi$ :

$$
u_{x}^{(1)}=u_{x^{\prime}}^{(1)} \cos \theta_{0}-u_{y^{\prime}}^{(1)} \operatorname{sen} \theta_{0} \Rightarrow \frac{\partial u_{x}^{(1)}}{\partial \xi}=\frac{\partial u_{x^{\prime}}^{(1)}}{\partial \xi} \cos \theta_{0}-\frac{\partial u_{y^{\prime}}^{(1)}}{\partial \xi} \operatorname{sen} \theta_{0}
$$

Uma vez que as variáveis $u_{x^{\prime}}^{(1)}, u_{y^{\prime}}^{(1)}$ são dependentes das coordenadas polares $\boldsymbol{r}$ e $\boldsymbol{\theta}$, as derivadas expressas por $\frac{\partial u_{x^{\prime}}^{(1)}}{\partial \xi}$ e $\frac{\partial u_{y^{\prime}}^{(1)}}{\partial \xi}$ podem ser obtidas mediante aplicação da regra da cadeia obtendo-se: 


$$
\begin{aligned}
\frac{\partial u_{x}^{(1)}}{\partial \xi} & =\frac{\partial u_{x^{\prime}}^{(1)}}{\partial \xi} \cos \theta_{0}-\frac{\partial u_{y^{\prime}}^{(1)}}{\partial \xi} \operatorname{sen} \theta_{0} \Rightarrow \\
& \Rightarrow \frac{\partial u_{x}^{(1)}}{\partial \xi}=\left\{\frac{\partial u_{x^{\prime}}^{(1)}}{\partial r} \frac{\partial r}{\partial \xi}+\frac{\partial u_{x^{\prime}}^{(1)}}{\partial \theta} \frac{\partial \theta}{\partial \xi}\right\} \cos \theta_{0}-\left\{\frac{\partial u_{y^{\prime}}^{(1)}}{\partial r} \frac{\partial r}{\partial \xi}+\frac{\partial u_{y^{\prime}}^{(1)}}{\partial \theta} \frac{\partial \theta}{\partial \xi}\right\} \operatorname{sen} \theta_{0}
\end{aligned}
$$

As derivadas de $u_{x^{\prime}}^{(1)}$ e $\quad u_{y^{\prime}}^{(1)}$ em relação às coordenadas polares $\boldsymbol{r}$ e $\boldsymbol{\theta}$ contidas em (6.50) são dadas por:

$$
\begin{aligned}
& \frac{\partial u_{x^{\prime}}^{(1)}}{\partial r}=\frac{\lambda_{1} r^{\lambda_{1}-1}}{2 G}\left\{\left[k-Q_{1}^{(1)}\left(\lambda_{1}+1\right)\right] \cos \left(\lambda_{1} \theta\right)-\lambda_{1} \cos \left(\left(\lambda_{1}-2\right) \theta\right)\right\} \\
& \frac{\partial u_{x^{\prime}}^{(1)}}{\partial \theta}=\frac{r^{\lambda_{1}}}{2 G}\left\{\left[k-Q_{1}^{(1)}\left(\lambda_{1}+1\right)\right]\left[-\lambda_{1} \operatorname{sen}\left(\lambda_{1} \theta\right)\right]+\lambda_{1}\left(\lambda_{1}-2\right) \operatorname{sen}\left(\left(\lambda_{1}-2\right) \theta\right)\right\} \\
& \frac{\partial u_{y^{\prime}}^{(1)}}{\partial r}=\frac{\lambda_{1} r^{\lambda_{1}-1}}{2 G}\left\{\left[k+Q_{1}^{(1)}\left(\lambda_{1}+1\right)\right] \operatorname{sen}\left(\lambda_{1} \theta\right)+\lambda_{1} \operatorname{sen}\left(\left(\lambda_{1}-2\right) \theta\right)\right\} \\
& \frac{\partial u_{y^{\prime}}^{(1)}}{\partial \theta}=\frac{r^{\lambda_{1}}}{2 G}\left\{\left[k+Q_{1}^{(1)}\left(\lambda_{1}+1\right)\right] \lambda_{1} \cos \left(\lambda_{1} \theta\right)+\lambda_{1}\left(\lambda_{1}-2\right) \cos \left(\left(\lambda_{1}-2\right) \theta\right)\right\}
\end{aligned}
$$

O próximo passo refere-se ao desenvolvimento das derivadas de $\boldsymbol{r}$ e $\boldsymbol{\theta}$ contidas em (6.50) em relação à coordenada paramétrica $\xi$. Como as variáveis $\boldsymbol{r}$ e $\boldsymbol{\theta}$ dependem das coordenadas $\mathrm{X}^{\prime}$ e $\mathrm{Y}^{\prime}$, aplica-se novamente a regra da cadeia:

$$
\begin{aligned}
\frac{\partial u_{x}^{(1)}}{\partial \xi} & =\left\{\frac{\partial u_{x^{\prime}}^{(1)}}{\partial r} \frac{\partial r}{\partial \xi}+\frac{\partial u_{x^{\prime}}^{(1)}}{\partial \theta} \frac{\partial \theta}{\partial \xi}\right\} \cos \theta_{0}-\left\{\frac{\partial u_{y^{\prime}}^{(1)}}{\partial r} \frac{\partial r}{\partial \xi}+\frac{\partial u_{y^{\prime}}^{(1)}}{\partial \theta} \frac{\partial \theta}{\partial \xi}\right\} \operatorname{sen} \theta_{0} \Rightarrow \\
\frac{\partial u_{x}^{(1)}}{\partial \xi}= & \left\{\frac{\partial u_{x^{\prime}}^{(1)}}{\partial r}\left[\frac{\partial r}{\partial x^{\prime}} \frac{\partial x^{\prime}}{\partial \xi}+\frac{\partial r}{\partial y^{\prime}} \frac{\partial y^{\prime}}{\partial \xi}\right]+\frac{\partial u_{x^{\prime}}^{(1)}}{\partial \theta}\left[\frac{\partial \theta}{\partial x^{\prime}} \frac{\partial x^{\prime}}{\partial \xi}+\frac{\partial \theta}{\partial y^{\prime}} \frac{\partial y^{\prime}}{\partial \xi}\right]\right\} \cos \theta_{0}-\cdots \\
& \cdots\left\{\frac{\partial u_{y^{\prime}}^{(1)}}{\partial r}\left[\frac{\partial r}{\partial x^{\prime}} \frac{\partial x^{\prime}}{\partial \xi}+\frac{\partial r}{\partial y^{\prime}} \frac{\partial y^{\prime}}{\partial \xi}\right]+\frac{\partial u_{y^{\prime}}^{(1)}}{\partial \theta}\left[\frac{\partial \theta}{\partial x^{\prime}} \frac{\partial x^{\prime}}{\partial \xi}+\frac{\partial \theta}{\partial y^{\prime}} \frac{\partial y^{\prime}}{\partial \xi}\right]\right\} \operatorname{sen} \theta_{0}
\end{aligned}
$$

Na equação (6.55) primeiramente faz-se necessário a obtenção das derivadas de $\boldsymbol{r}$ e $\boldsymbol{\theta}$ em relação às variáveis $x^{\prime}$ e $y^{\prime}$. Para isto, utilizam-se as seguintes relações de coordenadas polares: 


$$
\frac{\partial r}{\partial x^{\prime}}=\frac{x^{\prime}}{r}=\cos \theta \quad \frac{\partial r}{\partial y^{\prime}}=\frac{y^{\prime}}{r}=\operatorname{sen} \theta \quad \frac{\partial \theta}{\partial x^{\prime}}=-\frac{y^{\prime}}{r^{2}}=-\frac{\operatorname{sen} \theta}{r} \quad \frac{\partial \theta}{\partial y^{\prime}}=\frac{x^{\prime}}{r^{2}}=\frac{\cos \theta}{r}
$$

Como as variáveis $x^{\prime}$ e $y^{\prime}$ são dadas em função dos eixos $x$ e $y$, a equação (6.57) define a relação existente entre os eixos $\mathbf{X Y}$ e $\mathbf{X}^{\prime} \mathbf{Y}^{\prime}$ e consequientemente os valores de $x^{\prime}$ e $y^{\prime}$ definem-se por:

$$
\left\{\begin{array}{l}
x^{\prime}=x \cdot \cos \theta_{0}+y \cdot \operatorname{sen} \theta_{0} \\
y^{\prime}=-x \cdot \operatorname{sen} \theta_{0}+y \cdot \cos \theta_{0}
\end{array}\right.
$$

Nota-se que, a equação (6.57) é valida somente se a origem dos eixos $\mathbf{X Y}$ e $\mathbf{X}^{\prime} \mathbf{Y}^{\prime}$ coincidirem. Caso isto não ocorra, a equação (6.57) deve ser substituída por:

$$
\left\{\begin{array}{l}
x^{\prime}=\left(x-x_{0}\right) \cdot \cos \theta_{0}+\left(y-y_{0}\right) \cdot \operatorname{sen} \theta_{0} \\
y^{\prime}=-\left(x-x_{0}\right) \cdot \operatorname{sen} \theta_{0}+\left(y-y_{0}\right) \cdot \cos \theta_{0}
\end{array}\right.
$$

onde $(x, y)$ refere-se ao ponto $\boldsymbol{P}$ em questão e $\left(x_{0}, y_{0}\right)$ refere-se à coordenada global da ponta da fissura. Substituindo-se (6.58) em (6.56) obtêm-se então as derivadas de $\boldsymbol{r}$ e $\boldsymbol{\theta}$ em relação às variáveis $x^{\prime}$ e $y^{\prime}$ contidas na equação (6.55):

$$
\begin{aligned}
& \frac{\partial r}{\partial x^{\prime}}=\frac{x^{\prime}}{r}=\frac{\left(x-x_{0}\right) \cdot \cos \theta_{0}+\left(y-y_{0}\right) \cdot \operatorname{sen} \theta_{0}}{r} \\
& \frac{\partial r}{\partial y^{\prime}}=\frac{y^{\prime}}{r}=\frac{-\left(x-x_{0}\right) \cdot \operatorname{sen} \theta_{0}+\left(y-y_{0}\right) \cdot \cos \theta_{0}}{r}
\end{aligned}
$$

$\frac{\partial \theta}{\partial x^{\prime}}=-\frac{y^{\prime}}{r^{2}}=\frac{\left(x-x_{0}\right) \cdot \operatorname{sen} \theta_{0}-\left(y-y_{0}\right) \cdot \cos \theta_{0}}{r^{2}}$

$\frac{\partial \theta}{\partial y^{\prime}}=\frac{x^{\prime}}{r^{2}}=\frac{\left(x-x_{0}\right) \cdot \cos \theta_{0}+\left(y-y_{0}\right) \cdot \operatorname{sen} \theta_{0}}{r^{2}}$ 
Obtidas as derivadas escritas acima, aplica-se então a última seqüência da regra da cadeia, ou seja, realiza-se as derivadas das variáveis $x^{\prime}$ e $y^{\prime}$ (que estão em função de $x$ e $y$ ) em relação à variável paramétrica $\xi$. Partindo-se de (6.55), chega-se à seguinte equação:

$$
\begin{aligned}
& \frac{\partial u_{x}^{(1)}}{\partial \xi}=\left\{\frac{\partial u_{x^{\prime}}^{(1)}}{\partial r}\left[\frac{\partial r}{\partial x^{\prime}} \frac{\partial x^{\prime}}{\partial \xi}+\frac{\partial r}{\partial y^{\prime}} \frac{\partial y^{\prime}}{\partial \xi}\right]+\frac{\partial u_{x^{\prime}}^{(1)}}{\partial \theta}\left[\frac{\partial \theta}{\partial x^{\prime}} \frac{\partial x^{\prime}}{\partial \xi}+\frac{\partial \theta}{\partial y^{\prime}} \frac{\partial y^{\prime}}{\partial \xi}\right]\right\} \cos \theta_{0}-\cdots \\
& \cdots\left\{\frac{\partial u_{y^{\prime}}^{(1)}}{\partial r}\left[\frac{\partial r}{\partial x^{\prime}} \frac{\partial x^{\prime}}{\partial \xi}+\frac{\partial r}{\partial y^{\prime}} \frac{\partial y^{\prime}}{\partial \xi}\right]+\frac{\partial u_{y^{\prime}}^{(1)}}{\partial \theta}\left[\frac{\partial \theta}{\partial x^{\prime}} \frac{\partial x^{\prime}}{\partial \xi}+\frac{\partial \theta}{\partial y^{\prime}} \frac{\partial y^{\prime}}{\partial \xi}\right]\right\} \operatorname{sen} \theta_{0} \quad \Rightarrow \\
& \frac{\partial u_{x}^{(1)}}{\partial \xi}=\left\{\begin{array}{l}
\frac{\partial u_{x^{\prime}}^{(1)}}{\partial r}\left[\frac{\partial r}{\partial x^{\prime}}\left(\frac{\partial x^{\prime}}{\partial x} \frac{\partial x}{\partial \xi}+\frac{\partial x^{\prime}}{\partial y} \frac{\partial y}{\partial \xi}\right)+\frac{\partial r}{\partial y^{\prime}}\left(\frac{\partial y^{\prime}}{\partial x} \frac{\partial x}{\partial \xi}+\frac{\partial y^{\prime}}{\partial y} \frac{\partial y}{\partial \xi}\right)\right]+ \\
\frac{\partial u_{x^{\prime}}^{(1)}}{\partial \theta}\left[\frac{\partial \theta}{\partial x^{\prime}}\left(\frac{\partial x^{\prime}}{\partial x} \frac{\partial x}{\partial \xi}+\frac{\partial x^{\prime}}{\partial y} \frac{\partial y}{\partial \xi}\right)+\frac{\partial \theta}{\partial y^{\prime}}\left(\frac{\partial y^{\prime}}{\partial x} \frac{\partial x}{\partial \xi}+\frac{\partial y^{\prime}}{\partial y} \frac{\partial y}{\partial \xi}\right)\right]
\end{array}\right\} \cos \theta_{0}-\cdots \\
& \ldots\left\{\begin{array}{l}
\frac{\partial u_{y^{\prime}}^{(1)}}{\partial r}\left[\frac{\partial r}{\partial x^{\prime}}\left(\frac{\partial x^{\prime}}{\partial x} \frac{\partial x}{\partial \xi}+\frac{\partial x^{\prime}}{\partial y} \frac{\partial y}{\partial \xi}\right)+\frac{\partial r}{\partial y^{\prime}}\left(\frac{\partial y^{\prime}}{\partial x} \frac{\partial x}{\partial \xi}+\frac{\partial y^{\prime}}{\partial y} \frac{\partial y}{\partial \xi}\right)\right]+ \\
\frac{\partial u_{y^{\prime}}^{(1)}}{\partial \theta}\left[\frac{\partial \theta}{\partial x^{\prime}}\left(\frac{\partial x^{\prime}}{\partial x} \frac{\partial x}{\partial \xi}+\frac{\partial x^{\prime}}{\partial y} \frac{\partial y}{\partial \xi}\right)+\frac{\partial \theta}{\partial y^{\prime}}\left(\frac{\partial y^{\prime}}{\partial x} \frac{\partial x}{\partial \xi}+\frac{\partial y^{\prime}}{\partial y} \frac{\partial y}{\partial \xi}\right)\right]
\end{array}\right\} \operatorname{sen} \theta_{0}
\end{aligned}
$$

Na equação (6.63) as derivadas das variáveis $x^{\prime}$ e $y^{\prime}$ podem ser obtidas derivando-se (6.58) em relação às variáveis $x$ e $y$ :

$$
\left\{\begin{array}{l}
\frac{\partial x^{\prime}}{\partial x}=\frac{\partial\left[\left(x-x_{0}\right) \cdot \cos \theta_{0}+\left(y-y_{0}\right) \cdot \operatorname{sen} \theta_{0}\right]}{\partial x}=\cos \theta_{0} \\
\frac{\partial x^{\prime}}{\partial y}=\frac{\partial\left[\left(x-x_{0}\right) \cdot \cos \theta_{0}+\left(y-y_{0}\right) \cdot \operatorname{sen} \theta_{0}\right]}{\partial y}=\operatorname{sen} \theta_{0} \\
\frac{\partial y^{\prime}}{\partial x}=\frac{\partial\left[-\left(x-x_{0}\right) \cdot \operatorname{sen} \theta_{0}+\left(y-y_{0}\right) \cdot \cos \theta_{0}\right]}{\partial x}=-\operatorname{sen} \theta_{0} \\
\frac{\partial y^{\prime}}{\partial y}=\frac{\partial\left[-\left(x-x_{0}\right) \cdot \operatorname{sen} \theta_{0}+\left(y-y_{0}\right) \cdot \cos \theta_{0}\right]}{\partial y}=\cos \theta_{0}
\end{array}\right.
$$

Finalmente, as derivadas das variáveis $x$ e $y$ em relação à variável paramétrica $\xi$ são facilmente obtidas mediante a derivação da equação (6.64), que relaciona coordenadas cartesianas (globais) com as coordenadas paramétricas: 


$$
\begin{aligned}
& x=a_{0}+a_{1} \xi+a_{2} \eta+a_{3} \xi \eta \quad \Rightarrow \quad \frac{\partial x}{\partial \xi}=\frac{\partial\left(a_{0}+a_{1} \xi+a_{2} \eta+a_{3} \xi \eta\right)}{\partial \xi}=a_{1}+a_{3} \eta \\
& y=b_{0}+b_{1} \xi+b_{2} \eta+b_{3} \xi \eta \quad \Rightarrow \quad \frac{\partial y}{\partial \xi}=\frac{\partial\left(b_{0}+b_{1} \xi+b_{2} \eta+b_{3} \xi \eta\right)}{\partial \xi}=b_{1}+b_{3} \eta
\end{aligned}
$$

onde:

$$
\begin{cases}a_{0}=\frac{1}{4}\left(x_{1}+x_{2}+x_{3}+x_{4}\right) & b_{0}=\frac{1}{4}\left(y_{1}+y_{2}+y_{3}+y_{4}\right) \\ a_{1}=\frac{1}{4}\left(-x_{1}+x_{2}+x_{3}-x_{4}\right) & b_{1}=\frac{1}{4}\left(-y_{1}+y_{2}+y_{3}-y_{4}\right) \\ a_{2}=\frac{1}{4}\left(-x_{1}-x_{2}+x_{3}+x_{4}\right) & b_{2}=\frac{1}{4}\left(-y_{1}-y_{2}+y_{3}+y_{4}\right) \\ a_{3}=\frac{1}{4}\left(x_{1}-x_{2}+x_{3}-x_{4}\right) & b_{3}=\frac{1}{4}\left(y_{1}-y_{2}+y_{3}-y_{4}\right)\end{cases}
$$

Nas relações anteriores as variáveis $\left(x_{1}, y_{1}\right),\left(x_{2}, y_{2}\right),\left(x_{3}, y_{3}\right)$ e $\left(x_{4}, y_{4}\right)$ representam, respectivamente as coordenadas dos nós 1, 2, 3 e 4 do elemento finito isoparamétrico. É importante ressaltar que os procedimentos demonstrados até este ponto referem-se à derivada (em relação à variável paramétrica $\xi$ ) da função enriquecedora da direção global considerando-se o modo I de abertura (ver equação (6.49)). As derivadas das demais funções enriquecedoras (equações (6.45), (6.46) e (6.47)) em relação às coordenadas paramétricas $\xi$ e $\eta$ para os modos I e II de abertura podem ser obtidas utilizando-se dos mesmos procedimentos mencionados para a obtenção da equação (6.63). Mais aplicações destas funções enriquecedoras podem ser encontradas em Barros (2002), Oden e Duarte (1997) e Pereira (2004). 


\subsection{Exemplos Numéricos}

A seguir, apresentam-se exemplos numéricos com o objetivo de demonstrar a eficácia da técnica de enriquecimento da aproximação, utilizando-se tanto as auto-funções (solução analítica da fratura) apresentadas no item anterior como uma base de funções polinomiais. Primeiramente, o estudo do enriquecimento da aproximação na solução de exemplos denominados por problemas originais não se utiliza o Método da Partição. Posteriormente, nos outros exemplos, a ênfase é dada ao subproblema local do Método da Partição, procurando obter com o MEFG resultados suficientemente precisos com um número de graus de liberdade bem inferior àqueles exigidos pelo MEF. Exemplos da Mecânica da Fratura referentes aos modos I e II de abertura complementam o item.

\subsubsection{O Enriquecimento do Problema Original - Modo I de Abertura}

a) Chapa Contendo Uma Fissura - Carregamento Uniformemente Distribuído

Neste caso utiliza-se uma chapa com dimensões 10x10 (W=10 e H=10), comprimento de fissura $a=1$, e tensão constante unitária $(\sigma=1)$ aplicada ao longo da borda superior da chapa, que possui as seguintes características de material:

$$
\left\{\begin{array}{l}
G=1,0 \\
v=0,3
\end{array}\right.
$$

O raio " $\mathrm{r}$ " que define a região contendo todos os nós que serão enriquecidos é considerado igual a três. Este raio tem origem na ponta da fissura. A figura 6.5 ilustra os aspectos geométricos deste exemplo: 


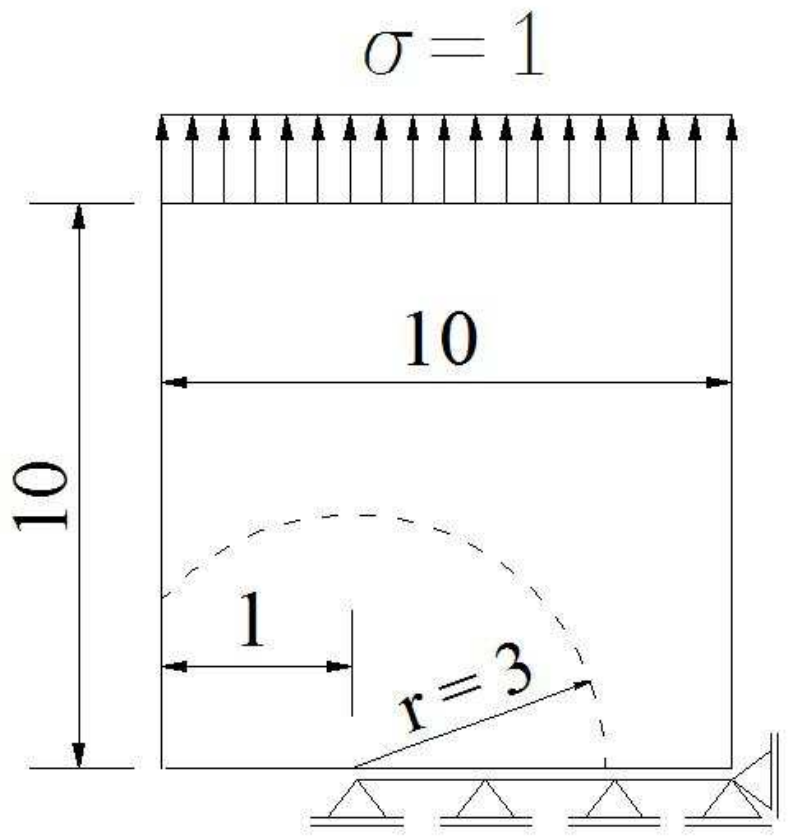

Figura 6.5 - Chapa Contendo Uma Fissura em Modo I de Abertura - Carregamento Constante

Primeiramente realiza-se uma análise convencional deste problema, via MEF, com uma malha bastante refinada. Tal malha possui 8310 elementos e 25107 nós, com um total de 50214 graus de liberdade nodais. $\mathrm{O}$ aspecto geral da malha está ilustrado na figura 6.6:

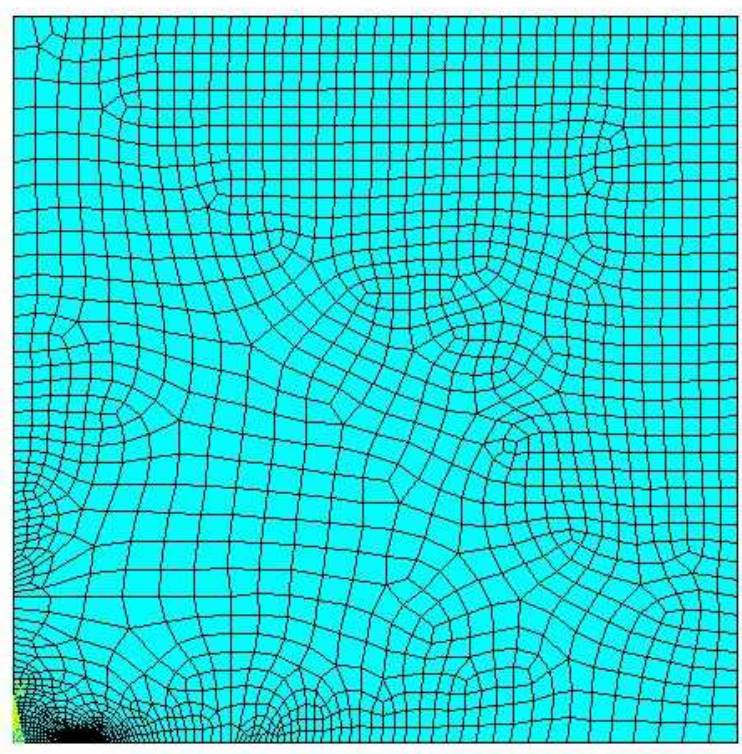

Figura 6.6 - Malha Refinada para o Caso de Uma Fissura 
A figura 6.7 apresenta os detalhes de refinamento da malha onde utilizam-se dois quadrantes contendo 60 elementos de lado. Tal malha permite a obtenção do fator de intensidade de tensão com uma precisão inferior a 1,0\%.

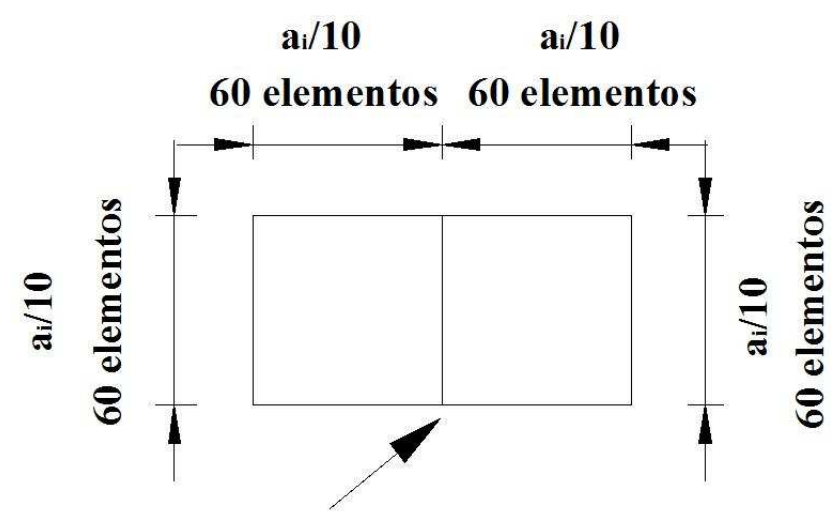

Ponta da fissura

Figura 6.7 - Detalhe do Refinamento na Ponta da Fissura

Em contrapartida, constrói-se uma malha com um número de nós bastante inferior ao da malha anterior contendo apenas 1835 nós e 576 elementos. O aspecto de tal malha é mostrado na figura 6.8. O objetivo é o de explorar a técnica de enriquecimento para obter um fator de intensidade de tensão com a mesma precisão, porém com um número de graus de liberdade nodais bem inferior ao da malha anterior.

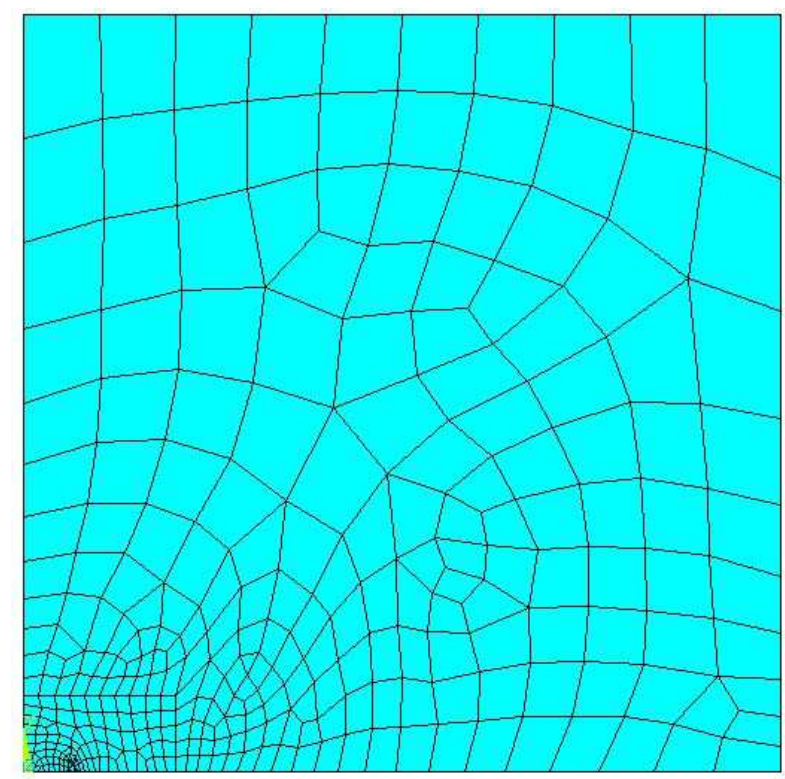

Figura 6.8 - Malha Graduada para o Caso de Uma Fissura 
As funções enriquecedoras da fratura utilizadas nas direções $\mathrm{X}$ e $\mathrm{Y}$ são respectivamente aquelas dadas pelas relações (6.40) e (6.41). Ambas as funções referem-se ao modo I de abertura (fissura horizontal):

$$
\begin{aligned}
& u_{x}^{(1)}=\frac{r^{\lambda_{1}}}{2 G}\left\{\left[k-Q_{1}^{(1)}\left(\lambda_{1}+1\right)\right] \cos \left(\lambda_{1} \theta\right)-\lambda_{1} \cos \left(\left(\lambda_{1}-2\right) \theta\right)\right\} \\
& u_{y}^{(1)}=\frac{r^{\lambda_{1}}}{2 G}\left\{\left[k+Q_{1}^{(1)}\left(\lambda_{1}+1\right)\right] \operatorname{sen}\left(\lambda_{1} \theta\right)+\lambda_{1} \operatorname{sen}\left(\left(\lambda_{1}-2\right) \theta\right)\right\}
\end{aligned}
$$

Estas funções enriquecedoras permitem capturar a parcela irregular da solução do problema referente à concentração de tensão na ponta da fissura. Já o enriquecimento por funções polinomiais têm o objetivo de capturar a parcela regular da solução do problema. Sendo assim, foram realizados vários testes com diferentes formas de enriquecimentos e os resultados de algumas combinações são dados na tabela 6.1 , onde indicam-se, também, os números de graus de liberdade (NGL) utilizados.

Tabela 6.1 - Valores de $\mathrm{K}_{\mathrm{I}}$ aproximados obtidos - via MEFG - Problema Original com 1 fissura

\begin{tabular}{|c|c|c|c|}
\hline \multicolumn{4}{|c|}{ 1 Fissura - Carregamento Constante : $\mathbf{K}_{\mathbf{R E F}}=\mathbf{2 , 0 9 8 1}$} \\
\hline Funções Enriquecedoras & $\mathbf{K}_{\text {APROx }}$ & $\mathbf{E}(\%)$ & NGL \\
\hline Nenhuma & 1,6887 & $-19,51$ & 3670 \\
\hline Polinomiais $\left[\mathrm{x}^{3}, \mathrm{y}^{3},\right]$ & 1,8318 & $-12,68$ & 6239 \\
\hline Fratura & 2,0568 & $-1,96$ & 6239 \\
\hline Polinomiais $\left[\mathbf{x}^{\mathbf{3}}, \mathbf{y}^{\mathbf{3}},\right]+$ Fratura & $\mathbf{2 , 0 7 6 7}$ & $\mathbf{- 1 , 0 4}$ & $\mathbf{8 8 0 8}$ \\
\hline
\end{tabular}

Com base nos resultados obtidos verifica-se que a melhor estratégia de enriquecimento refere-se àquela onde combinam-se as funções da fratura, com as funções polinomiais. Foi necessário um número bem inferior de graus de liberdade em relação àquele utilizado na abordagem convencional, apenas 17,5\% . 
b) Chapa Contendo Duas Fissuras - Carregamento Uniformemente Distribuído

A chapa mostrada na figura 6.9 possui as mesmas características de material e geometria do exemplo anterior, porém com uma segunda fissura horizontal (de comprimento unitário) no lado direito da chapa. $\mathrm{O}$ raio “ $\mathrm{r}$ " que define a região de enriquecimento tem o mesmo valor do exemplo anterior (igual a 3).

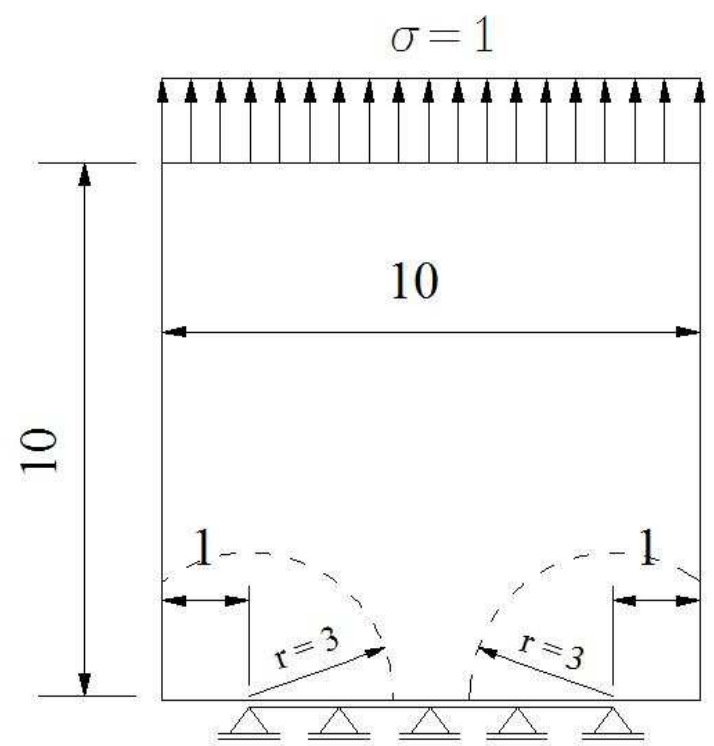

Figura 6.9 - Chapa Contendo Duas Fissuras em Modo I de Abertura - Carregamento Constante

Para a análise convencional deste problema, via MEF, constrói-se uma malha bastante refinada, ilustrada na figura 6.10. Tal malha possui 29177 elementos e 87988 nós, com um total de 175976 graus de liberdade nodais. A característica de refinamento na ponta da fissura é idêntica à da malha do exemplo anterior.

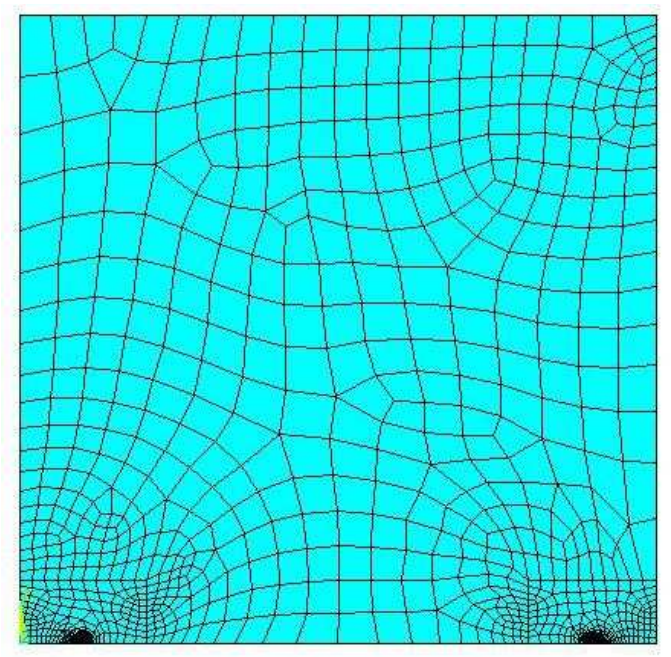

Figura 6.10 - Malha Refinada para o Caso de Duas Fissuras 
Para a utilização do MEFG foi construída uma malha bem menos refinada possuindo 910 elementos e 2875 nós (5750 graus de liberdade), conforme ilustram as figuras 6.11 e 6.12 .

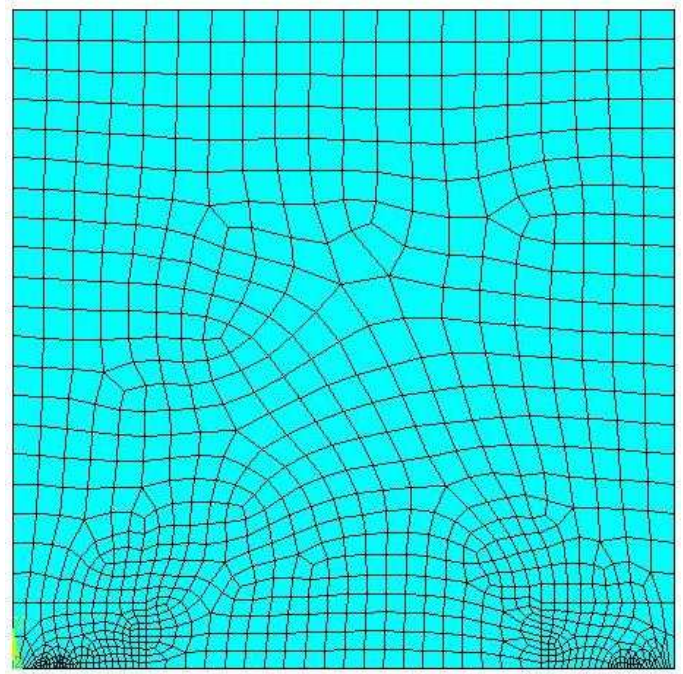

Figura 6.11 - Malha Graduada para o Caso de Duas Fissuras

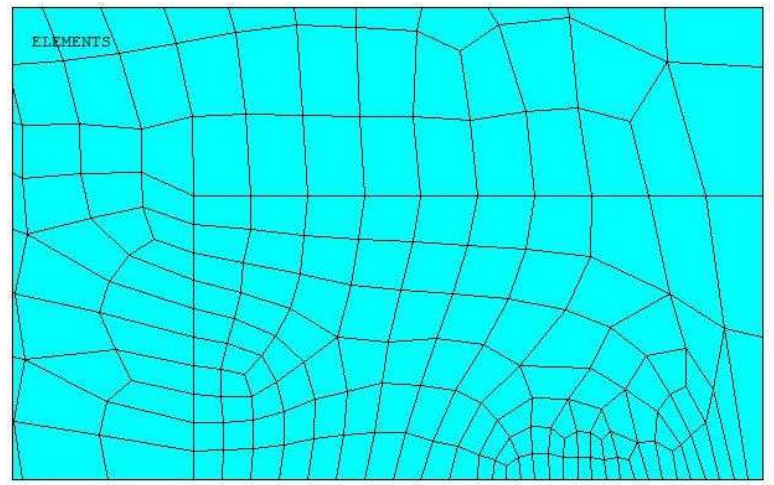

Figura 6.12 - Detalhe da Malha Graduada na Região da Ponta de Fissura - Modo I

Os valores obtidos com base no enriquecimento polinomial aliado ao enriquecimento com as funções da fratura são resumidos na tabela a 6.2 .

Tabela 6.2 - Valores de $\mathrm{K}_{\mathrm{I}}$ aproximados obtidos - via MEFG - Problema Original com 2 fissuras

\begin{tabular}{|c|c|c|c|}
\hline \multicolumn{4}{|c|}{ 2 Fissuras - Carregamento Constante : $K_{\text {REF }}=1,9884$} \\
\hline Funções Enriquecedoras & K $_{\text {APROx }}$ & E(\%) & NGL \\
\hline Nenhuma & 1,8571 & $-6,60$ & 5750 \\
\hline Polinomiais $\left[\mathrm{x}^{3}, \mathrm{y}^{3},\right]+$ Fratura & 1,9662 & $-1,10$ & 14375 \\
\hline
\end{tabular}


Assim como no exemplo anterior, verificou-se que o enriquecimento utilizando as funções polinomiais e da fratura foram capazes de propiciar resultado com mesma precisão daquele apresentado com malha muito refinada $(\approx 1 \%)$. Foram necessários apenas $8,2 \%$ dos graus de liberdade utilizados com a malha refinada.

Os próximos itens referem-se à análise do subproblema local do Método da Partição. O objetivo é alcançar bons resultados do subproblema local com uma malha bem menos refinada e conseqüentemente com um custo computacional bem mais reduzido em relação à abordagem convencional pelo MEF.

6.5.2 Método da Partição com Enriquecimento do Subproblema Local - Modo I de Abertura

a) Análise de Chapa Contendo Duas Fissuras - Carregamento Uniformemente Distribuído. A figura 6.13 representa o caso em estudo:

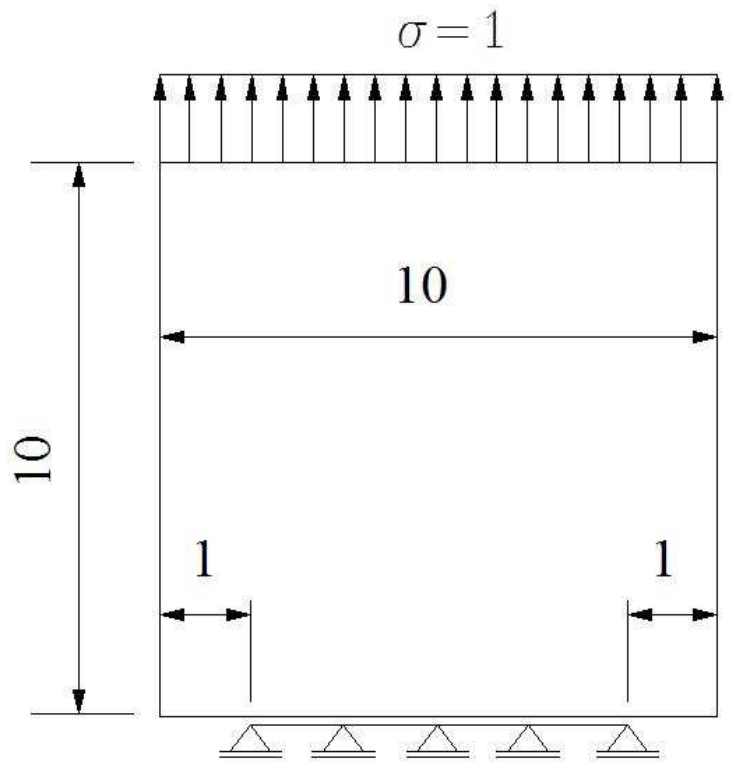

Figura 6.13 - Chapa Contendo Duas Fissuras em Modo I de Abertura - Carregamento Constante

O atual exemplo consiste na resolução de um caso do item anterior (chapa contendo duas fissuras), porém através da utilização do Método da Partição. Neste novo procedimento, 
o problema original é dividido em três subproblemas, sendo que a técnica de enriquecimento é utilizada apenas no subproblema local $\boldsymbol{P}_{L}^{(k)}$. Sendo assim, faz-se necessário comentar sobre a discretização das faces de fissura de $\boldsymbol{P}_{L}^{(k)}$. Como no problema original as forças externas estão aplicadas na borda superior da chapa, não há a necessidade de uma discretização considerável nas faces de fissuras. Em contrapartida, ao se utilizar o Método da Partição, fazse necessário uma discretização razoável das faces de fissura do subproblema $\boldsymbol{P}_{L}^{(k)}$, uma vez que as mesmas estão submetidas aos fluxos de tensão $\boldsymbol{Q}_{j}$ (base polinomial aproximativa). O objetivo de tal discretização é retratar com uma boa precisão a ação destes fluxos de tensão assim como o comportamento de abertura das faces de fissura.

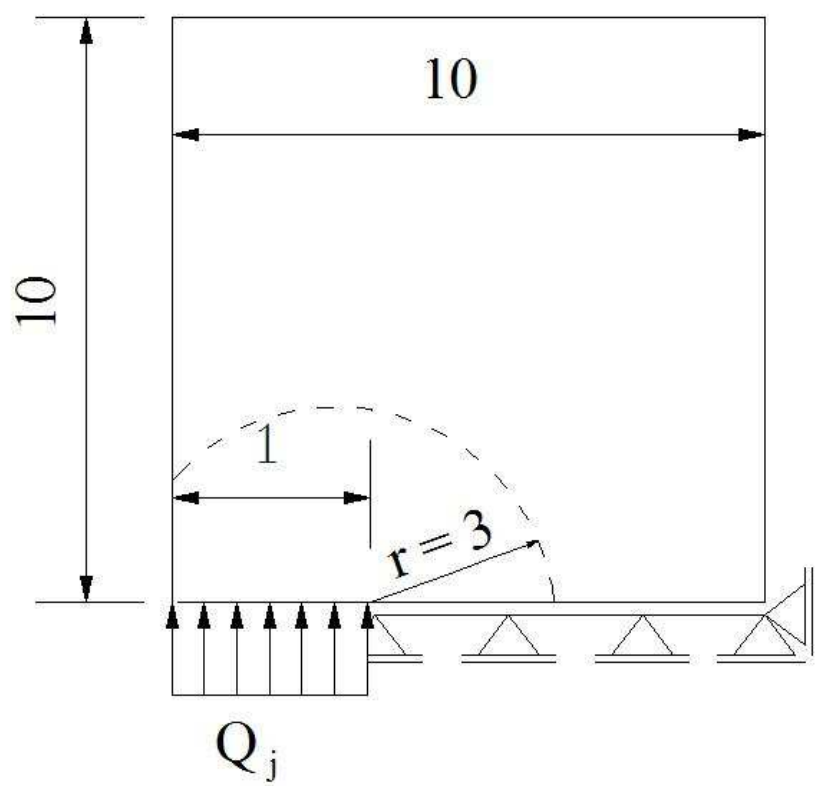

Figura 6.14 - Dimensões do Subproblema Local - Modo I de Abertura

Como o carregamento externo é constante, somente 1 termo da aproximação do campo de tensão na linha de fissura é necessário para que o Método da Partição possa capturar a solução do problema. Com isto, um único subproblema $\boldsymbol{P}_{L}^{(k)}$ faz-se necessário, sendo o carregamento $\boldsymbol{Q}_{j}$, aplicado na face da fissura, considerado unitário. Sobre a técnica de enriquecimento, os mesmos procedimentos adotados nos exemplos do item anterior foram seguidos, além da mesma malha graduada para 1 fissura (figura 6.8). Os resultados obtidos são resumidos na tabela 6.3 . 
Tabela 6.3 - Valores de $K_{I}$ aproximados obtidos - via MEFG - Método da Partição com 2 fissuras

\begin{tabular}{|c|c|c|c|}
\hline \multicolumn{3}{|c|}{2 Fissuras - Carregamento Constante : $\mathbf{K}_{\mathbf{R E F}}=\mathbf{1 , 9 8 8 4}$} \\
\hline Funções Enriquecedoras & $\mathbf{K}_{\text {APROx }}$ & $\mathbf{E}(\%)$ & NGL \\
\hline Nenhuma & 1,6161 & $-18,72$ & 3670 \\
\hline Polinomiais $\left[\mathrm{x}, \mathrm{x}^{2}, \mathrm{y}, \mathrm{y}^{2}\right]$ & 1,7219 & $-13,40$ & 6239 \\
\hline Fratura & 1,9571 & $-1,57$ & 6239 \\
\hline Polinomiais $\left[\mathrm{x}, \mathrm{x}^{2}, \mathrm{y}, \mathrm{y}^{2}\right]+$ Fratura & $\mathbf{1 , 9 7 3 8}$ & $\mathbf{- 0 , 7 3}$ & $\mathbf{8 8 0 8}$ \\
\hline
\end{tabular}

Assim como nos exemplos anteriores, a técnica oferecida pelo MEFG propiciou resultados bastante satisfatórios, utilizando-se somente $17,5 \%$ dos graus de liberdade necessários daqueles utilizados em malhas refinadas. Para ambos os casos a precisão se manteve abaixo de $1 \%$ em relação à solução analítica do fator de intensidade de tensão.

O próximo exemplo trata do mesmo caso, porém o carregamento externo possui variação quadrática. Desta maneira, busca-se realizar uma análise da efetividade do enriquecimento dos subproblemas locais que possuirão carregamentos $\boldsymbol{Q}_{j}$ constante, linear e quadrático.

b) Análise de Chapa Contendo Duas Fissuras - Carregamento Não-Uniforme. A figura 6.15 ilustra este caso:

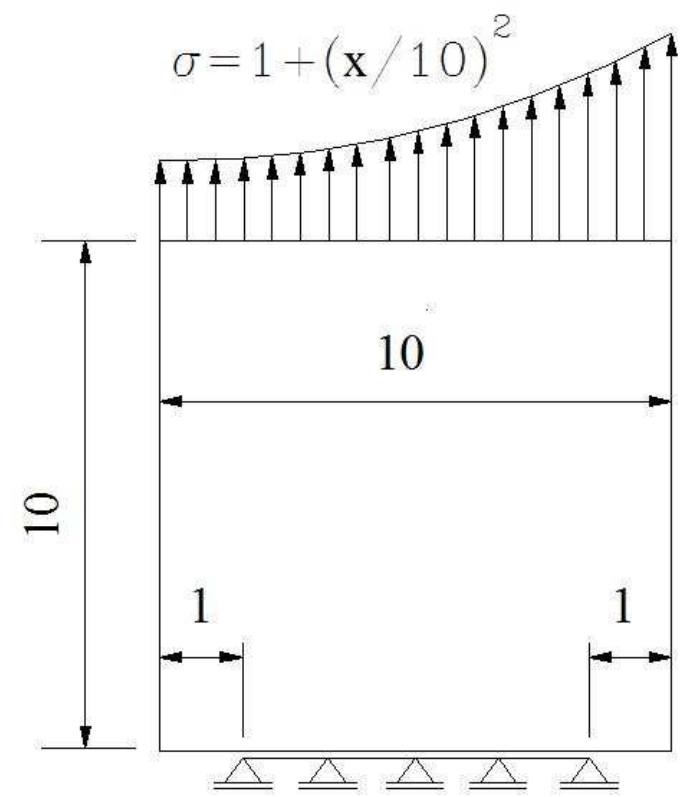

Figura 6.15 - Chapa Contendo Duas Fissuras em Modo I de Abertura - Carregamento Cte+Quadrático 
Neste exemplo, as mesmas estratégias de enriquecimento foram utilizadas nos três subproblemas locais, caracterizados por carregamentos nas faces das fissuras dos tipos: constante, linear e quadrático. Sendo assim, um aspecto bastante interessante refere-se aos subproblemas locais $\boldsymbol{P}_{L}^{(2)}$ e $\boldsymbol{P}_{L}^{(3)}$. Como evidencia a tabela 6.4, o tipo de enriquecimento que propiciou melhores resultados foi o polinomial. Tal fato se deve a que os carregamentos $\boldsymbol{Q}_{j}$ para os subproblemas $\boldsymbol{P}_{L}^{(2)}$ e $\boldsymbol{P}_{L}^{(3)}$ (linear e quadrático) possuírem valores nulos na ponta da fissura e unitários somente na raiz da fissura. Sendo assim, a concentração de tensão na ponta da fissura se torna bem menos intensa do que aquela existente no subproblema local $\boldsymbol{P}_{L}^{(\mathbf{1})}$. Neste, devido ao carregamento constante, a tensão aplicada na ponta da fissura é unitária e se estende com este valor até a raiz da fissura.

Tabela 6.4 - Valores de $K_{\mathrm{I}}$ aproximados obtidos - via MEFG - Subproblemas Locais

\begin{tabular}{|c|c|c|c|c|c|c|}
\hline \multirow{2}{*}{ Subproblema Local } & \multicolumn{2}{c|}{$\begin{array}{c}\text { PL (1) } \\
\text { PL (2) }\end{array}$} & \multicolumn{2}{c|}{ PL (3) } \\
& \multicolumn{2}{|c|}{$K_{\text {REF }}=\mathbf{2 , 0 9 8 1}$} & \multicolumn{2}{c|}{ K $_{\text {REF }}=\mathbf{0 , 8 5 6}$} & \multicolumn{2}{c|}{ K $_{\text {REF }}=\mathbf{0 , 5 5 6}$} \\
\hline F. Enriquecedoras & $K_{\text {APROx }}$ & E(\%) & $K_{\text {APROx }}$ & E(\%) & K $_{\text {APROx }}$ & E(\%) \\
\hline Nenhuma & 1,8121 & $-13,63$ & 0,8073 & $-5,24$ & 0,5145 & $-7,46$ \\
\hline Polinomiais $\left[\mathrm{x}, \mathrm{x}^{2}, \mathrm{y}, \mathrm{y}^{2}\right]$ & 1,9518 & $-6,97$ & $\mathbf{0 , 8 6 5}$ & $\mathbf{1 , 5 2}$ & $\mathbf{0 , 5 5 0 7}$ & $\mathbf{- 0 , 9 3}$ \\
\hline Fratura & 2,0708 & $-1,30$ & 0,9152 & 7,42 & 0,5834 & 4,94 \\
\hline Polinomiais $\left[\mathrm{x}, \mathrm{x}^{2}, \mathrm{y}, \mathrm{y}^{2}\right]+$ Fratura & $\mathbf{2 , 0 8 5 0}$ & $\mathbf{- 0 , 6 2}$ & 0,9167 & 7,60 & 0,5834 & 4,93 \\
\hline
\end{tabular}

Na tabela 6.5 são apresentados os valores dos fatores de intensidade de tensão finais obtidos aplicando-se enriquecimento polinomial dos subproblemas $\boldsymbol{P}_{L}^{(2)}$ e $\boldsymbol{P}_{L}^{(3)}$ e enriquecimento combinado, polinomial e da fratura, para o subproblema $\boldsymbol{P}_{L}^{(\mathbf{1})}$. Como a malha utilizada para os subproblemas locais refere-se à malha graduada para 1 fissura do item anterior, o número de graus de liberdade nodais se manteve em 17,5\% daqueles utilizados em analises via MEF convencional (com refinamento de malha). 
Tabela 6.5 - Valores de $K_{I}$ aproximados obtidos - via MEFG - Método da Partição com 2 fissuras

\begin{tabular}{|c|c|c|c|c|c|}
\hline \multicolumn{6}{|c|}{ Enriquecimento : PL(1)=Polinomial + Fratura ; PL(2 e 3)=Polinomial } \\
\hline \multicolumn{3}{|c|}{ Fissura 1: $K_{\mathrm{REF}}=1,633$} & \multicolumn{3}{|c|}{ Fissura 2: $K_{\mathrm{REF}}=3,642$} \\
\hline $\begin{array}{c}\mathbf{N}^{0} \text { termos } \\
\text { Aprox. }\end{array}$ & $\mathbf{K}_{\mathrm{APROX}}$ & $\mathbf{E}(\%)$ & $\begin{array}{c}\mathrm{N}^{0} \text { termos } \\
\text { Aprox. }\end{array}$ & $\mathbf{K}_{\text {APROX }}$ & $\mathbf{E}(\%)$ \\
\hline 1 & 3,5149 & $-3,49$ & 1 & 1,7480 & 7,04 \\
\hline 2 & 3,7035 & 1,69 & 2 & 1,5270 & $-6,49$ \\
\hline 3 & 3,6167 & 0,69 & 3 & 1,6235 & 0,58 \\
\hline
\end{tabular}

6.5.3 Método da Partição com Enriquecimento do Subproblema Local - Modo II de Abertura

a) Análise de Chapa Contendo Duas Fissuras - Carregamento Não-Uniforme. A figura 6.16 ilustra as características deste exemplo:

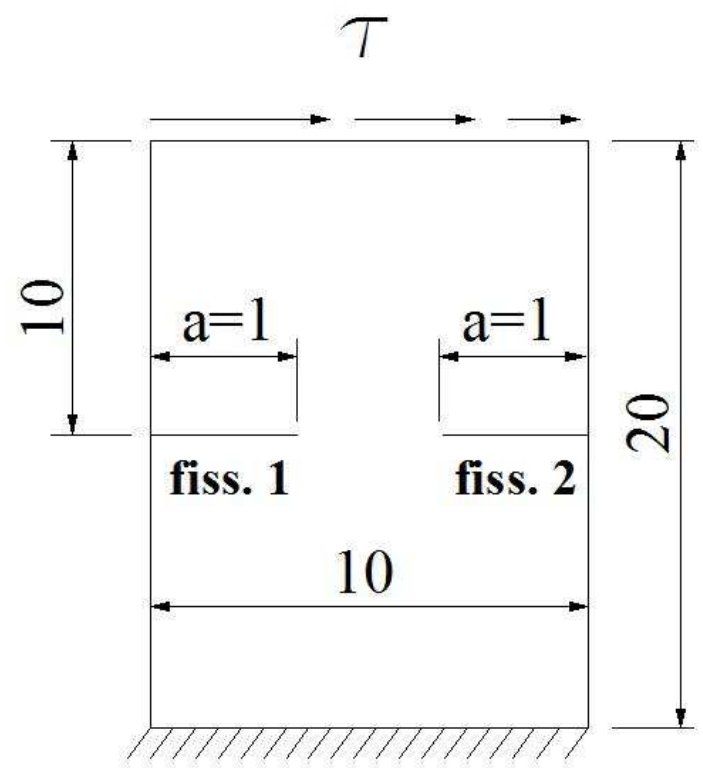

Figura 6.16 - Chapa Contendo Duas Fissuras em Modo II de Abertura - Carregamento Constante

Neste caso, utiliza-se uma chapa com dimensões 10x20 (W=10 e H=20), comprimento de fissura $\mathrm{a}=1$, e tensão constante+quadrática aplicada ao longo da borda superior da chapa. As seguintes características do material foram adotadas: 
$G=1,0$

$v=0,3$

Na figura 6.17 são apresentadas as dimensões do subproblema local.

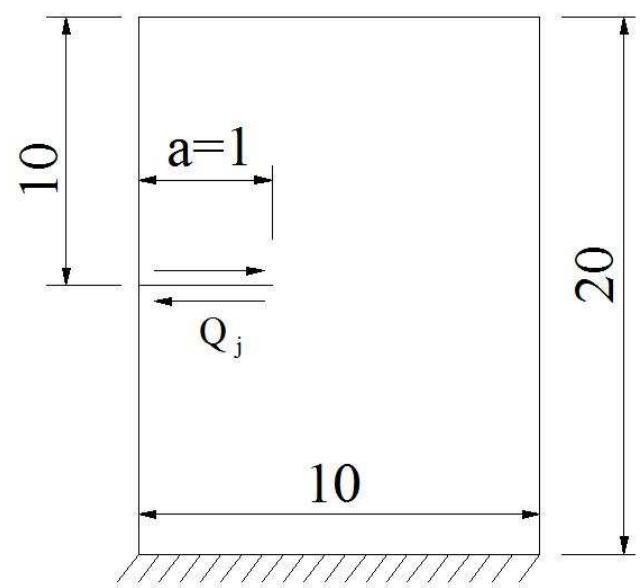

Figura 6.17 - Dimensões do Subproblema Local - Modo II de Abertura

Com o objetivo de realizar a extração dos fatores de intensidade de tensão dos subproblemas locais via MEF convencional, opta-se pela utilização de uma malha contendo 15219 elementos e 44363 nós (88726 graus de liberdade nodais), onde o critério de refinamento na região adjacente à ponta da fissura obedece os critérios do item 6.5.1. A figura 6.18 mostra a malha adotada:

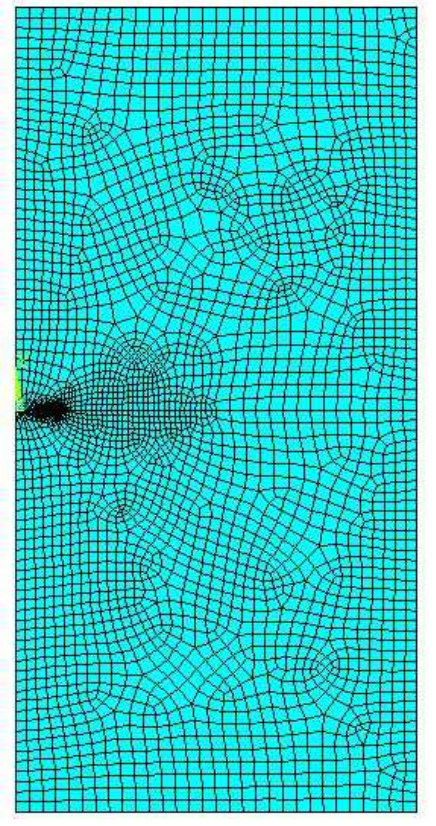

Figura 6.18 - Malha Refinada para o Subproblema Local - Modo II 
Para a utilização da técnica de enriquecimento via MEFG, utilizou-se uma malha graduada contendo somente 455 elementos e 525 nós (1050 graus de liberdade nodais) para o subproblema local $\boldsymbol{P}_{L}^{(k)}$. As figuras 6.19 e 6.20 ilustram as malhas dos subproblemas locais:

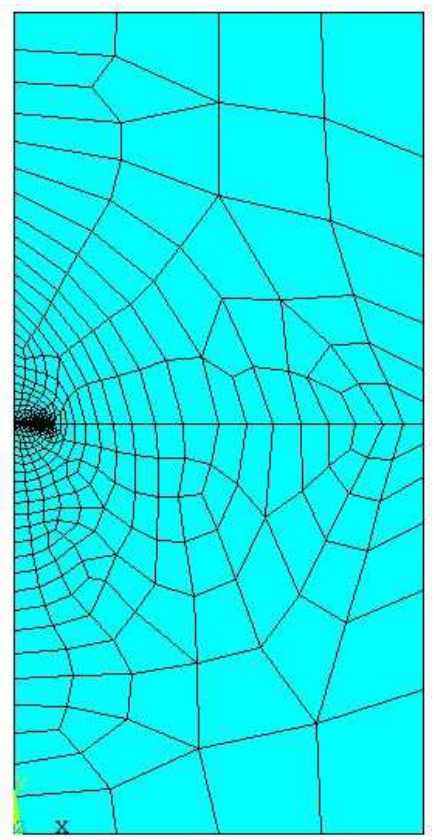

Figura 6.19 - Malha Graduada para o Subproblema Local - Modo II

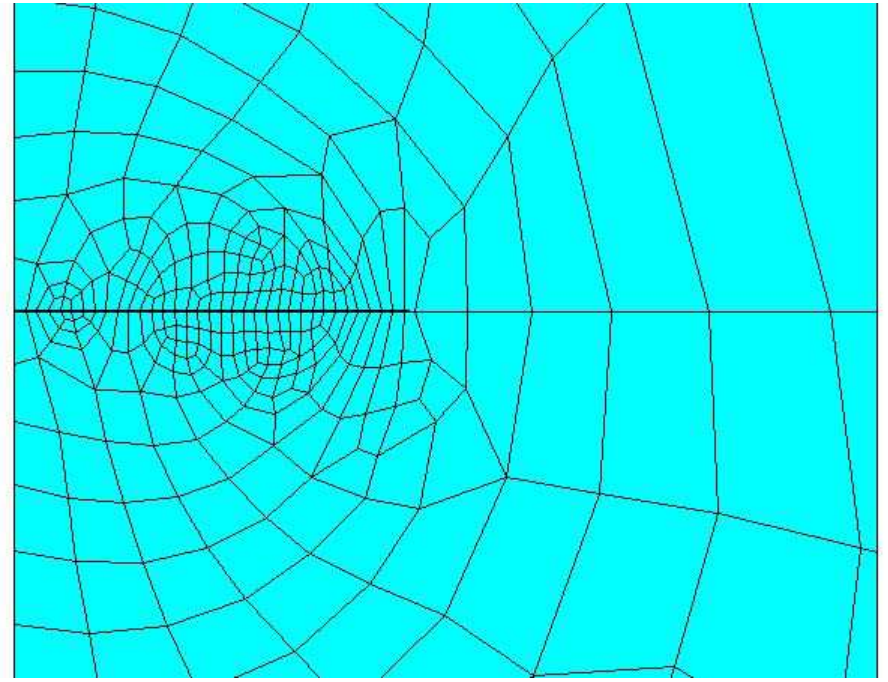

Figura 6.20 - Detalhe da Malha Graduada na Região da Ponta de Fissura - Modo II 
Aplicando-se a técnica de enriquecimento, foram obtidos resultados com a mesma precisão (aprox. 1\%) daqueles obtidos via MEF convencional (malha refinada), porém com um número de graus de liberdade bastante inferior (aproximadamente 10,2\% daqueles utilizados nas analises via MEF convencional). Neste exemplo o Método da Partição necessitou de 3 termos de aproximação e em todos os subproblemas foram utilizadas as mesmas bases polinomiais $\left(\mathrm{x}, \mathrm{x}^{2}, \mathrm{y}, \mathrm{y}^{2}\right)$ adicionadas das seguintes funções enriquecedoras da fratura para as direções $\mathrm{X}$ e $\mathrm{Y}$ respectivamente:

$$
\begin{aligned}
& u_{x}^{(2)}=\frac{r^{\lambda_{1}}}{2 G}\left\{\left[k-Q_{1}^{(2)}\left(\lambda_{1}+1\right)\right] \operatorname{sen}\left(\lambda_{1} \theta\right)-\lambda_{1} \operatorname{sen}\left(\left(\lambda_{1}-2\right) \theta\right)\right\} \\
& u_{y}^{(2)}=\frac{-r^{\lambda_{1}}}{2 G}\left\{\left[k+Q_{1}^{(2)}\left(\lambda_{1}+1\right)\right] \cos \left(\lambda_{1} \theta\right)+\lambda_{1} \cos \left(\left(\lambda_{1}-2\right) \theta\right)\right\}
\end{aligned}
$$

A tabela 6.6 reúne os resultados obtidos:

Tabela 6.6 - Valores de $\mathrm{K}_{\mathrm{II}}$ aproximados obtidos - via MEFG - Método da Partição com 2 fissuras

\begin{tabular}{|c|c|c|c|c|c|}
\hline \multicolumn{3}{|c|}{ Fissura 1: $\mathbf{K}_{\text {REF }}=\mathbf{0 , 8 8 2}$} & \multicolumn{3}{c|}{ Fissura 2: $\mathbf{K}_{\mathbf{R E F}}=\mathbf{0 , 9 0 4}$} \\
\hline $\begin{array}{c}\mathbf{N}^{\mathbf{0}} \text { termos } \\
\text { Aprox. }\end{array}$ & $\mathbf{K}_{\text {APROX }}$ & $\mathbf{E}(\%)$ & $\begin{array}{c}\mathbf{N}^{\mathbf{0}} \text { termos } \\
\text { Aprox. }\end{array}$ & $\mathbf{K}_{\text {APROx }}$ & $\mathbf{E}(\%)$ \\
\hline 2 & 0,8579 & $-2,73$ & 2 & 0,8774 & $-2,72$ \\
\hline 3 & 0,8788 & $-0,36$ & 3 & 0,9012 & $-0,31$ \\
\hline
\end{tabular}




\section{Considerações Finais e Conclusões}

Neste trabalho foram realizadas aplicações do Método da Partição objetivando a análise plana de problemas com múltiplas fissuras. Com relação ao método foram detalhadamente estudados e implementados alguns procedimentos preliminares fundamentais, entre eles destacando-se: a análise da base de funções para a representação do campo de tensões em uma linha de interesse e a determinação de fatores de intensidade de tensão.

É importante ressaltar que o procedimento adotado para a extração dos fatores de intensidade de tensão foi desenvolvido a partir de uma análise muito criteriosa; isto porque a precisão do Método da Partição quanto à solução final do problema é sensível a quaisquer variações nos valores de $\mathrm{K}$ dos problemas locais.

Os testes realizados com uma única fissura revelaram que o terceiro subproblema do Método da Partição, $P_{G}^{(k)}$, se implementado de acordo com a versão apresentada na referência original de Andersson, Babuška e Stehlin (1998), proporcionava erro na solução. Lembra-se que o objetivo deste subproblema, no caso geral de múltiplas fissuras, é o de reproduzir a 
influência do campo de tensão de uma fissura sobre as demais. Seguindo a proposição original, considerava-se também a influência de uma dada fissura sobre si mesma, o que é inconsistente. Depois de observado este erro, corrigiu-se o procedimento e, com isto, as respostas passaram a ser coerentes com os valores previstos analiticamente.

Em seguida, realizaram-se testes com a presença de duas fissuras, tomando-se o cuidado de corrigir o terceiro subproblema a fim de se avaliar a influência de uma fissura em relação à outra. Os testes apresentaram respostas, relativas ao fator de intensidade de tensão, com alta precisão e taxa de convergência exponencial na medida em que se tomaram mais termos aproximativos dos campos de tensão nas linhas das fissuras do subproblema $P_{G}^{(0)}$.

Outra classe de problemas testada tem motivação nas aplicações aeronáuticas relacionadas à integridade estrutural, como as ligações rebitadas dos elementos de revestimento de asas e fuselagens. Em tais problemas as fissuras podem se propagar por solicitação repetida a partir dos furos dos rebites e a possibilidade de interligação entre as diversas fissuras pode, naturalmente, comprometer a integridade de toda a ligação. No exemplo testado, considerou-se uma fissura com origem em furo circular, representando o furo do rebite. Numericamente o problema torna-se mais complexo porque o furo provoca perturbações iniciais significativas no campo de tensão do primeiro (e mais simples) subproblema do Método da Partição, e que devem ser aproximadas com precisão pela base de aproximação adotada.

Nos testes dessa classe de problemas, a base polinomial, inicialmente proposta no procedimento, mostrou-se limitada para a boa aproximação do campo de tensões nas linhas das fissuras. A base alternativa que proporcionou melhor resultado contêm a classe das racionais e foi capaz de recuperar a convergência exponencial para a solução exata do Método da Partição. No teste realizado nessa classe de problemas, cinco termos da base irracional proporcionaram uma solução aproximada com erro inferior a $0,5 \%$.

Outra classe de problemas explorados refere-se a chapas contendo fissuras internas. Como a formulação proposta por Andersson, Babuška e Stehlin (1998) contempla somente fissuras de borda, procurou-se estendê-la para a abordagem de situações contendo fissuras internas e de borda-furo. Neste sentido foram realizados exemplos contendo ambos os tipos de fissuras buscando-se desenvolver uma metodologia para a montagem da matriz de influência geral que permite a obtenção dos fatores de escala dos subproblemas que combinados propiciam a solução do problema. Os exemplos realizados, com a obtenção de 
resultados com alta precisão (em torno de 1\%), demonstraram a eficácia da metodologia do Método da Partição estendida aos casos contendo diversos tipos de fissuras.

Em uma última etapa, empregou-se a técnica de enriquecimento da aproximação fornecida pelo Método dos Elementos Finitos Generalizados (MEFG) na resolução dos subproblemas locais do Método da Partição. O enriquecimento seletivo da aproximação atrelada a determinados nós, sem necessidade de alteração nem refinamento excessivo da rede de elementos finitos adotada, proporciona melhores resultados com menor custo computacional. No caso, os nós selecionados para o enriquecimento referem-se àqueles das regiões adjacentes às pontas e faces de fissuras. Nos exemplos explorados, comprovou-se que a técnica de enriquecimento da aproximação propicia resultados com precisão similar àqueles obtidos via MEF convencional (malhas refinadas), porém com uma malha bem menos refinada e um número de graus de liberdade nodais bastante inferior. Um aspecto específico, porém importante a ressaltar, refere-se às estratégias de enriquecimento para os diferentes subproblemas locais (cada subproblema atrelado a um termo da base de aproximação). Notouse que o primeiro termo da aproximação (constante) proporciona uma concentração de tensão considerável na ponta da fissura, o que exige, no enriquecimento, além das funções polinomiais também as funções-solução da fratura. Já os demais termos da aproximação (linear, quadrático, etc.) proporcionam baixa concentração de tensão na região da ponta de fissura, sendo suficientes as funções enriquecedoras polinomiais. Concluindo, a combinação MEFG com o Método da Partição reduz o custo computacional preservando um ótimo nível de precisão.

Finalmente, entende-se que o presente trabalho abre claras perspectivas para a sua continuidade, inseridas no campo da Mecânica do Multi-Fraturamento. Entre as alternativas de extensão imediata estão aquelas de cunho computacional e de melhoria da modelagem e aproximação numérica, como por exemplo: a paralelização dos processamentos relativos aos problemas locais, a consideração de solicitações repetidas, a abordagem tridimensional e sua combinação com procedimentos de propagação de fissuras. 


\section{Referências Bibliográficas}

ALLIABADI, M. H.; ROOKE, D. P. (1991). Numerical fracture mechanics. Dordrecht: Kluwer Academic Publishers.

ALPA, G.; BOCCA, P.; CARPINTERI, A.; et al (1992). Mecanica de material e della frattura. Bologna: Pitagora Editrice s.r.l. .

ALVES, M. M. (2005). Emprego do método de resíduos ponderados para a análise de tubos. Dissertação (Mestrado). Escola de Engenharia de São Carlos, Universidade de São Paulo.

ANDERSON, T. L. (1994). Fracture mechanics: fundamentals and applications. 2.ed. CRC Press LCC. 
ANDERSSON, B.; BABUSKA, I.; STEHLIN, P. (1998). Reliable 3D multiple-site fracture mechanics analysis. The Aeronautical Research Institute of Sweden, document number: FFA TN - 1998-18.

ASSAN, A. E. (2003). Método dos elementos finitos: primeiros passos. 2.ed. Campinas: Editora da Unicamp.

ATLURI, S. N. (1986). Computational methods in the mechanics of fracture - Volume 2. Elsevier Science Publishers B. V.

BABUŠKA, I.; CALOZ, G.; OSBORN, J. E. (1994). Special finite element method for a classe of second order elliptic problems with rough coefficients. SIAM Journal on Numerical Analysis, vol. 31, n. 4, p. 727-981.

BABUSKA, I.; MELENK, J. M. (1997). The partition of unity method. International Journal for Numerical Methods in Engineering, v. 40, p. 727-758.

BABUSKA I.; ANDERSSON, B. (2005). The splitting method as a tool for multiple damage analysis. SIAM: Journal on Scientific Computing, vol. 26, n. 4, p. 1114-1145.

BARROS, F. B. (2002). Métodos sem malha e método dos elementos finitos generalizados em análise não-linear de estruturas. Tese (Doutorado). Escola de Engenharia de São Carlos, Universidade de São Paulo.

BATHE, K. J. (1996). Finite elements procedures. 2.ed. New Jersey: Prentice-Hall.

BITTENCOURT, T. N.; MIRANDA, A. C. O.; MEGIOLARO, M. A. et al (2003). Fatigue life and crack path predictions in generic 2D structural components. Engineering Fracture Mechanics, v. 70, p. 1259-1279.

BORTMAN, Y.; BANKS-SILLS, L. (1983). An extended weight function method for mixedmode elastic crack analysis. Journal of Applied Mechanics, vol. 50, p. 907-909. 
BOSE FILHO, W. W. (2006). Introdução a Mecânica da Fratura. Notas de Aula. Escola de Engenharia de São Carlos, Universidade de São Paulo.

BRANCO, C. M.; FERNANDES, A. A.; DE CASTRO, P. M. S. T. (1999). Fadiga de estruturas soldadas. 2.ed. Lisboa: Fundação Calouste Gulbenkian.

BROEK, D (1986). Elementary engineering fracture mechanics. 4.ed. Dordrecht: Kluwer Academic Publishers.

BUECKNER, H. F. (1958). The propagation of cracks and the energy of elastic deformation. Trans. ASME, n. 80, p. 1225-1230.

BUECKNER, H. F. (1970). A novel principle for the computation of stress intensity factors. ZAMM - Journal od Applied Mathematics and Mechanics / Zeitschrift für Angewandte Mathematik und Mechanik. Vol. 50, n. 4, p. 526-546.

BURNS, D. J.; GLINKA, G.; KICIAK, A. (2003). Calculation of Stress Intensity Factors and Crack Opening Displacements for Cracks Subjected to Complex Stress Fields. ASME \& Journal of Pressure Vessel Technology, vol. 125 (august), p. 260-265.

CARVALHO, C. V. A. (1998). Simulação bidimensional adaptativa por elementos finitos de processos de fraturamento por fadiga. Dissertação (Mestrado). Escola de Engenharia de São Carlos, Universidade de São Paulo.

CHELL, G. G. (1976). The Stress Intensity Factors and Crack Profiles for Centre and Edge Cracks in Plates Subjected to Arbitrary Stresses. International Journal of Fracture, vol. 12, p. 33-46.

DUARTE, C. A.; ODEN, J. T. (1995). Hp clouds - a meshless to solve boundary - value problem. The University of Texas at Austin. Technical Report. TICAM.

DUARTE, C. A. (1996). The hp-cloud method. Tese (Doutorado). The University of Texas at Austin. 
DUARTE, C. A.; ODEN, J. T. (1996). Hp clouds - an hp meshless method. Numerical Methods for Partial Differential Equations. John Wiley \& Sons, p. 1-34.

DUARTE, C. A.; BABUSKA, I.; ODEN, J. T. (2000). Generalized finite element methods for three-dimensional structural mechanics problems. Computers \& Structures, v. 77, n. 2 p. 215-232.

EDWALDS, H.L. WANHILL, R.J.H., (1986). Fracture mechanics. Delftse Uitgevers Maatschapij.

FERRANTE, M. (2002). Seleção de materiais. 2.ed. São Carlos: Ed. UFSCar.

FERREIRA, L. E. T. (2005). Tópicos especiais de engenharia de estruturas : mecânica do fraturamento aplicada à engenharia de estruturas. Notas de Aula. Escola de Engenharia de São Carlos, Universidade de São Paulo.

GDOUTOS, E. E., (1993). Fracture mechanics - an introduction. Dordrecht: Kluwer Academic Publishers.

GÓIS, W. (2004). Método dos elementos finitos generalizados em formulação variacional mista. Dissertação (Mestrado). Escola de Engenharia de São Carlos, Universidade de São Paulo.

GRANDT JÚNIOR, A. F. (1978). Stress intensity factors for cracked holes and rings loaded with polynomial crack face pressure distributions. International Journal of Fracture, vol. 14, n. 4, p. 221-229.

GRIFFITH, A. A. (1924). The theory of rupture. In: I INTERNATIONAL CONGRESS FOR APPLIED, 1924, Delft. Proceedings... Delft, C. B. BIEZENO \& BURGERS, J. M., 1925, p. $55-63$.

HSU, T. M.; RUDD, J. L. (1977). Green's function for thru-crack emanating from fastener holes. In: INTERNATIONAL CONFERENCE ON FRACTURE. 1977, Waterloo, Canada. Proceedings... Waterloo, Canada, D. M. R. TAPLIN, 1977, vol. 3. 
INGLIS, C. E. (1913). Stresses in a plate due to the presence of cracks and sharp corners. Transactions of the Institution of Naval Architects, vol. 55, p. 219-241.

IRWIN, G. (1957). Analysis of stresses and strain near the end of a crack traversing a plate. Journal of Applied Mechanics, vol. 24, p. 361-364.

JONES, D. S. (1974). Integral equations for the exterior acoustic problem Q J. Journal of Mechanics and Applied Mathematics, vol. 27, p. 129-142.

KAYA, A. C.; ERDOGAN, F. (1980). Stress intensity factor and COD in an orthotropic strip. International Journal of Fracture, vol. 16, p. 171-190.

LACHENBRUCH, A. H. (1961). Depth and spacing of tension cracks. Journal of Geophysical Research, vol. 66, p. 4273-4292.

LEONEL, E. D. (2006). Método dos elementos de contorno aplicado à análise de sólidos multi-fraturados. Dissertação (Mestrado). Escola de Engenharia de São Carlos, Universidade de São Paulo.

MEGUID, S. A. (1989). Engineering fracture mechanics. New York: Elsevier Science Publishers LTD.

MELENK, J. M. (1995). On generalized finite element methods. Tese (Doutorado). University of Maryland, College Park.

MELENK, J. M.; BABUSKA, I. (1996). The partition of unity finite element method: basic theory and applications. Computer Methods in Applied Mechanics and Engineering, v. 139, p. 289-314.

NAKASONE, N.; STOLARSKI, T. A., YOSHIMOTO, S. (2006). Engineeging analysis with ANSYS Software. Oxford: Elsevier Butterworth-Heinemann. 
NISHIOKA, T.; ATLURI, S. N. (1983). Analytical solution for embedded elliptical cracks, and finite element alternating method for elliptical surface cracks, subject to arbitrary loadings. Engineering Fracture Mechanics, vol. 17, n. 3, p. 247-268.

ODEN, J. T.; DUARTE, C. A. (1997). Clouds, cracks and fem's. In: INTERNATIONAL CENTER OF NUMERICAL METHODS IN ENGINEERING, 1997, Barcelona, Spain. Proceedings... Barcelona, Spain, REDDY, B. D., Recent developments in computational and applied mechanics and engineering, 1997, p. 302-321.

ODEN, J. T.; DUARTE, C. A. (1997). Solution of singular problems using hp clouds. In: THE MATHEMATICS OF FINITE ELEMENTS AND APPROXIMATIONS. 1997, New York. Proceedings... New York, WHITEMAN, J. R., 1997, p. 35-54.

ODEN, J. T.; DUARTE, C. A.; ZIENKIEWICZ, O. C. (1998). A new cloud - based hp finite element method. Computer Methods in Applied Mechanics and Engineering. vol. 153, p. 117-126.

OWEN, D. R. J.; FAWKES, A. J. (1983). Engineering fracture mechanics. Swansea: Pineridge Press Ltd.

PEREIRA, J. P. A. (2004). Extração de fatores de intensidade de tensão utilizando a solução do método dos elementos finitos generalizados. Dissertação (Mestrado). Escola de Engenharia de São Carlos, Universidade de São Paulo.

PROENÇA, S. P. B. (2008). Introdução aos métodos numéricos. Notas de Aula. Escola de Engenharia de São Carlos, Universidade de São Paulo.

PROPPE, C. (2003). Probabilistic Analysis of Multi-Site Damage in Aircraft Fuselagens. Computational Mechanics, n. 30, p. 323-329.

RICE, J. R. (1972). Some remarks on elastic crack-tip fields. International Journal of Solids and Structures, vol. 8, p. 751-758. 
ROOKE, D. P.; CARTWRIGHT, D. J. (1977). Compendium of stress intensity factors. London: H.M.S.O.

ROOKE, D. P.; TWEED, J. (1980). Stress intensity factors for a crack at the edge of pressurized hole. International Journal of Engineering Science, vol. 18, p. 109-121.

ROOKE, D. P.; HUTCHINS, S. M. (1984). Stress intensity factors for cracks at loaded holeseffect of load distribution. The Journal of Strain Analysis for Engineering Design, vol. 19, n. 2, p. 81-96.

SAVASSI, W. (1996). Introdução aos elementos finitos em análise linear de estruturas. São Carlos: Escola de Engenharia de São Carlos, Universidade de São Paulo.

SHAM, T. L. (1989). The theory of higher order weight functions for linear elastic plane problems. International Journal of Solids and Structures, vol. 25, p. 357-380.

SHILOV, G. E. (1996). Elementary real and complex analysis. New York: Dover.

SHIVAKUMAR, V.; FORMAN, R. G. (1980). Green's function for a crack emanating from a circular hole. in an infinite sheet. International Journal of Fracture, vol. 16, p. 305-316.

STEPHEN, J. M. (2001). Fractures and faults in the earth. Lecture Notes. School of Ocean and Earth Science and Technology, University of Hawaii.

STROUBOULIS, T.; BABUŠKA, I.; COPS, K. (2000). The design and analysis of the generalized finite element method. Computer Methods in Applied Mechanics and Engineering, vol. 181, n. 1-3, p. 43-69.

SURESH, S. (2001). Fatigue of materials. 2.ed. Cambridge: The Press Syndicate of the University of Cambridge.

SZABÓ, B.; BABUŠKA, I. (1991). Finite Element Analysis. John Wiley \& Sons, Inc. 
TADA, H.; PARIS, P.; IRWIN, G. (2000). The stress analysis of crack handbook. 3.ed. New York: The American Society of Mechanical Engineers.

TIMOSHENKO, S. P.; GOODIER, J. N. (1980). Teoria da elasticidade. Guanabara Dois S. A.

TORRES, I. R. F. (2003). Desenvolvimento e aplicação do método dos elementos finitos generalizados em análise tridimensional não-linear de sólidos. Tese (Doutorado). Escola de Engenharia de São Carlos, Universidade de São Paulo.

WANG, L.; ATLURI, S. N. (1996). Recent advances in the alternating method for elastic and inelastic fracture analyses. Computer Methods in Applied Mechanics and Engineering, vol. 137 , p. 1-58.

WANG, L.; BRUST, F. W.; ATLURI, S. N. (1997). The elastic-plastic finite element alternating method (EPFEAM) and the prediction of fracture under WFD conditions in aircraft structures. Part I: EPFEAM theory. Computational Mechanics, n. 19, p. 356-369.

WESTERGAARD, H. M. (1939). Bearing pressures and cracks. Journal of Applied Mechanics, vol. 6, p. 49-53.

ZIENKIEWICZ, O. C. (2000). The finite element method - Volume 1. : The basis. 5.ed. Butterworth Heinemann. 
Apêndice A

Equação Bi-Harmônica 
Thimoshenko e Goodier (1980) apresentam a função tensão de Airy $\psi$ utilizada na solução de problemas da elasticidade bidimensional, sendo as tensões expressas da seguinte forma:

$$
\sigma_{x}=\frac{\partial^{2} \psi}{\partial y^{2}} \quad \sigma_{y}=\frac{\partial^{2} \psi}{\partial x^{2}} \quad \tau_{x y}=-\frac{\partial^{2} \psi}{\partial x \cdot \partial y}
$$

Tendo em vista que as equações que definem a compatibilidade entre deslocamentos e deformações de um dado problema elástico são expressas da seguinte maneira:

$$
\varepsilon_{x}=\frac{\partial u_{x}}{\partial x} \quad \varepsilon_{y}=\frac{\partial u_{y}}{\partial y} \quad \gamma_{x y}=\left(\frac{\partial u_{x}}{\partial y}+\frac{\partial u_{y}}{\partial x}\right)
$$

Pode-se então relacionar a equação (A.1) com a equação (A.2) por meio da Lei de Hooke expressa abaixo:

$$
\varepsilon_{x}=\frac{1}{E}\left(\sigma_{x}-v \cdot \sigma_{y}\right) \quad \varepsilon_{y}=\frac{1}{E}\left(\sigma_{y}-v \cdot \sigma_{x}\right) \quad \gamma_{x y}=2\left(\frac{1+v}{E}\right) \cdot \tau_{x y}
$$

Ou seja, inserindo a função tensão de Airy (A.1) e as deformações expressas por suas derivadas direcionais (A.2) nas equações definidas por (A.3) tem-se:

$$
\begin{array}{ll}
\varepsilon_{x}=\frac{1}{E}\left(\sigma_{x}-v \cdot \sigma_{y}\right) \quad \Rightarrow \quad \frac{\partial u_{x}}{\partial x}=\frac{1}{E} \cdot\left(\frac{\partial^{2} \psi}{\partial y^{2}}-v \cdot \frac{\partial^{2} \psi}{\partial x^{2}}\right) \\
\varepsilon_{y}=\frac{1}{E}\left(\sigma_{y}-v \cdot \sigma_{x}\right) & \Rightarrow \frac{\partial u_{y}}{\partial y}=\frac{1}{E} \cdot\left(\frac{\partial^{2} \psi}{\partial x^{2}}-v \cdot \frac{\partial^{2} \psi}{\partial y^{2}}\right) \\
\gamma_{x y}=\left(\frac{1+v}{E}\right) \cdot \tau_{x y} & \Rightarrow\left(\frac{\partial u_{x}}{\partial y}+\frac{\partial u_{y}}{\partial x}\right)=2\left(\frac{1+v}{E}\right) \cdot\left(-\frac{\partial^{2} \psi}{\partial x \partial y}\right)
\end{array}
$$


Realizando então as derivadas das equações de (A.4) conforme abaixo obtêm-se:

$$
\begin{gathered}
\frac{\partial u_{x}}{\partial x}=\frac{1}{E} \cdot\left(\frac{\partial^{2} \psi}{\partial y^{2}}-v \cdot \frac{\partial^{2} \psi}{\partial x^{2}}\right) \Rightarrow \frac{\partial^{2}}{\partial y^{2}} \Rightarrow \quad \frac{\partial^{3} u_{x}}{\partial x \partial y^{2}}=\frac{1}{E} \cdot\left(\frac{\partial^{4} \psi}{\partial y^{4}}-v \cdot \frac{\partial^{4} \psi}{\partial x^{2} \partial y^{2}}\right) \\
\frac{\partial u_{y}}{\partial y}=\frac{1}{E} \cdot\left(\frac{\partial^{2} \psi}{\partial x^{2}}-v \cdot \frac{\partial^{2} \psi}{\partial y^{2}}\right) \Rightarrow \frac{\partial^{2}}{\partial x^{2}} \Rightarrow \quad \frac{\partial^{3} u_{y}}{\partial y \partial x^{2}}=\frac{1}{E} \cdot\left(\frac{\partial^{4} \psi}{\partial x^{4}}-v \cdot \frac{\partial^{4} \psi}{\partial x^{2} \partial y^{2}}\right) \\
\left(\frac{\partial u_{x}}{\partial y}+\frac{\partial u_{y}}{\partial x}\right)=2\left(\frac{1+v}{E}\right) \cdot\left(-\frac{\partial^{2} \psi}{\partial x \partial y}\right) \Rightarrow \frac{\partial^{2}}{\partial x \partial y} \Rightarrow \quad\left(\frac{\partial^{3} u_{x}}{\partial x \partial y^{2}}+\frac{\partial^{3} u_{y}}{\partial y \partial x^{2}}\right)=2\left(\frac{1+v}{E}\right) \cdot\left(-\frac{\partial^{4} \psi}{\partial x^{2} \partial y^{2}}\right)
\end{gathered}
$$

Bastando inserir as equações (A.5.a) e (A.5.b) na equação (A.5.c) obtêm-se a equação biharmônica representativa dos problemas bidimensionais da elasticidade plana:

$$
\begin{gathered}
\left(\frac{\partial^{3} u_{x}}{\partial x \partial y^{2}}+\frac{\partial^{3} u_{y}}{\partial y \partial x^{2}}\right)=2\left(\frac{1+v}{E}\right) \cdot\left(-\frac{\partial^{4} \psi}{\partial x^{2} \partial y^{2}}\right) \Rightarrow \\
\frac{1}{2} \cdot\left[\frac{1}{\Sigma} \cdot\left(\frac{\partial^{4} \psi}{\partial y^{4}}-v \cdot \frac{\partial^{4} \psi}{\partial x^{2} \partial y^{2}}\right)+\frac{1}{\Sigma} \cdot\left(\frac{\partial^{4} \psi}{\partial x^{4}}-v \cdot \frac{\partial^{4} \psi}{\partial x^{2} \partial y^{2}}\right)\right]=\left(\frac{1+v}{\Sigma}\right) \cdot\left(-\frac{\partial^{4} \psi}{\partial x^{2} \partial y^{2}}\right) \Rightarrow \frac{\partial^{4} \psi}{\partial y^{4}}+\frac{\partial^{4} \psi}{\partial x^{4}}=-2 \cdot \frac{\partial^{4} \psi}{\partial x^{2} \partial y^{2}} \Rightarrow \\
\frac{\partial^{4} \psi}{\partial y^{4}}+\frac{\partial^{4} \psi}{\partial x^{4}}-\left(2 v \cdot \frac{\partial^{4} \psi}{\partial x^{2} \partial x^{2}}\right)=-2(1+\downarrow) \cdot \frac{\partial^{4} \psi}{\partial x^{2} \partial y^{2}} \Rightarrow \\
\Rightarrow \frac{\partial^{4} \psi}{\partial y^{4}}+2 \cdot \frac{\partial^{4} \psi}{\partial x^{2} \partial y^{2}}+\frac{\partial^{4} \psi}{\partial x^{4}}=0
\end{gathered}
$$




\section{Apêndice B}

Condição de Cauchy-Riemann 
A condição de Cauchy-Riemann pode ser obtida realizando-se a verificação da continuidade da função analítica de uma variável complexa:

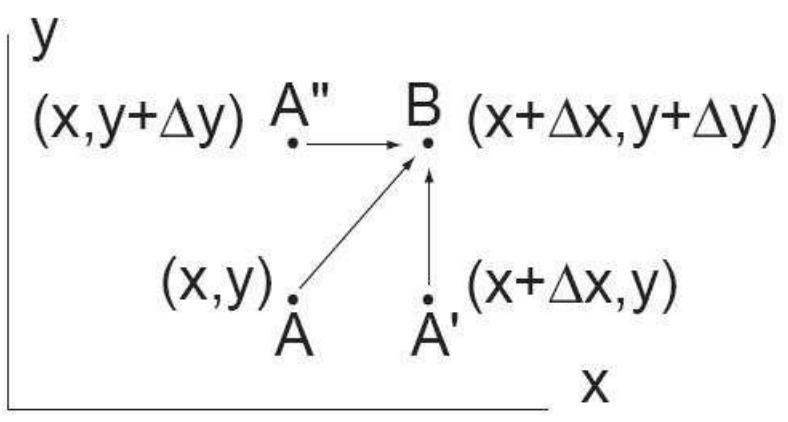

Figura B.1 - Condição de Cauchy-Riemann

$Z=Z(z) \quad \therefore z=f(x, y) \quad \Rightarrow \quad Z=Z(x, y)$

$Z(x, y)=\operatorname{Re} Z(x, y)+i \cdot \operatorname{Im} Z(x, y)$

Seguindo a definição de derivada parcial em um ponto, aplica-se então este conceito à função acima, tanto em relação à direção $x$ quanto à direção $y$, obtendo as seguintes expressões:

$$
\begin{aligned}
& \frac{\partial Z(x, y)}{\partial x}=\lim _{h \rightarrow 0} \frac{Z(x+h, y)-Z(x, y)}{h} \\
& \frac{\partial Z(x, y)}{\partial x}=\left[\lim _{h \rightarrow 0} \frac{\operatorname{Re} Z(x+h, y)+i \cdot \operatorname{Im} Z(x+h, y)}{h}\right]-\left[\lim _{h \rightarrow 0} \frac{\operatorname{Re} Z(x, y)+i \cdot \operatorname{Im} Z(x, y)}{h}\right] \\
& \frac{\partial Z(x, y)}{\partial x}=\left[\lim _{h \rightarrow 0} \frac{\operatorname{Re} Z(x+h, y)-\operatorname{Re} Z(x, y)}{h}\right]+i \cdot\left[\lim _{h \rightarrow 0} \frac{\operatorname{Im} Z(x+h, y)-\operatorname{Im} Z(x, y)}{h}\right] \\
& \frac{\partial Z(x, y)}{\partial x}=\frac{\partial \operatorname{Re} Z(x, y)}{\partial x}+i \cdot \frac{\partial \operatorname{Im} Z(x, y)}{\partial x} \Rightarrow Z^{\prime}=\frac{\partial \operatorname{Re} Z}{\partial x}+i \cdot \frac{\partial \operatorname{Im} Z}{\partial x}
\end{aligned}
$$




$$
\begin{aligned}
& \frac{\partial Z(x, y)}{d y}=\lim _{h \rightarrow 0} \frac{Z(x, y+h)-Z(x, y)}{h i} \\
& \frac{\partial Z(x, y)}{\partial y}=\left[\lim _{h \rightarrow 0} \frac{\operatorname{Re} Z(x, y+h)+i \cdot \operatorname{Im} Z(x, y+h)}{h i}\right]-\left[\lim _{h \rightarrow 0} \frac{\operatorname{Re} Z(x, y)+i \cdot \operatorname{Im} Z(x, y)}{h i}\right] \\
& \frac{\partial Z(x, y)}{\partial y}=\left[\lim _{h \rightarrow 0}\left(\frac{\operatorname{Re} Z(x, y+h)-\operatorname{Re} Z(x, y)}{h}\right) \cdot(-i)\right]+\left[\lim _{h \rightarrow 0} \frac{\operatorname{Im} Z(x, y+h)-\operatorname{Im} Z(x, y)}{h}\right] \\
& \frac{\partial Z(x, y)}{\partial y}=\frac{\partial \operatorname{Re} Z(x, y)}{\partial y} \cdot(-i)+\frac{\partial \operatorname{Im} Z(x, y)}{\partial y} \Rightarrow Z^{\prime}=-i \cdot \frac{\partial \operatorname{Re} Z}{\partial y}+\frac{\partial \operatorname{Im} Z}{\partial y}
\end{aligned}
$$

Shilov (1996) indica a função $Z(x, y)$ como sendo uma função complexa diferenciável, ou seja, possuindo a primeira derivada parcial contínua na vizinhança do ponto em questão (ponto A conforme figura B.1). Com isto, o valor da derivada de $Z(x, y)$ deve ser o mesmo para qualquer orientação. Desta maneira iguala-se as expressões (B.3) e (B.4) obtendo:

$$
Z^{\prime}=\underbrace{\underbrace{\frac{\partial \operatorname{Re} Z}{\partial x}}_{\text {parte real }}+i \cdot \underbrace{\frac{\partial \operatorname{Im} Z}{\partial x}}_{\text {parte imaginária }}}_{\text {equação }}=\underbrace{\frac{\partial \operatorname{Im} Z}{\partial y}}_{\text {equação B.4 }}+i \cdot \frac{\underbrace{\frac{\partial(-\operatorname{Re} Z)}{\partial y}}_{\text {parte real imaginária }}}{\underbrace{\frac{\partial \operatorname{lm}}{\partial y}}_{\text {parte }}}
$$

Sendo $Z^{\prime}=\operatorname{Re} Z^{\prime}+i \cdot \operatorname{Im} Z^{\prime}$

e observando que o primeiro membro das expressões igualadas em (B.5) equivale à parte real de $Z^{\prime}$ e que o segundo membro de cada uma destas expressões equivale à parte imaginária de $Z^{\prime}$, pode-se então igualar as partes real e imaginária de (B.5). Sendo assim, obtêm-se as duas expressões que definem a condição de Cauchy-Riemann:

$\operatorname{Re} Z^{\prime}=\frac{\partial \operatorname{Re} Z}{\partial x}=\frac{\partial \operatorname{Im} Z}{\partial y}$

$\operatorname{Im} Z^{\prime}=\frac{\partial \operatorname{Im} Z}{\partial x}=-\frac{\partial \operatorname{Re} Z}{\partial y}$ 


\section{Apêndice C}

\section{Funções Complexas de Westergaard}


O objetivo deste apêndice é a demonstração de que as funções $\left(\psi_{I}, \psi_{I I}\right)$ expressas por (2.18) e (2.19) representam a solução de problemas clássicos da Mecânica da Fratura, Stephen (2001). Em primeiro lugar apresenta-se então a seguinte função bi-harmônica $\psi$ através de uma combinação de funções harmônicas $\Omega_{i}$. Tal função $\psi$ é a "candidata" à solução da equação bi-harmônica (2.2), uma vez que as condições de contorno do problema (modo I de abertura) referem-se à cota $y=0$ (ver fig. 2.9):

$\psi=\Omega_{0}+\Omega_{1} \cdot y$

Posteriormente realizam-se as derivadas de (C.1) com o intuito de se obter as tensões baseando-se na função tensão de Airy.

$$
\begin{aligned}
& \frac{\partial \psi}{\partial x}=\frac{\partial\left(\Omega_{0}+\Omega_{1} \cdot y\right)}{\partial x}=\frac{\partial \Omega_{0}}{\partial x}+\frac{\partial\left(\Omega_{1} \cdot y\right)}{\partial x}=\frac{\partial \Omega_{0}}{\partial x}+\left(\frac{\partial \Omega_{1}}{\partial x} \cdot y+\Omega_{y} \frac{\partial y}{\partial x}\right)=\frac{\partial \Omega_{0}}{\partial x}+\frac{\partial \Omega_{1}}{\partial x} \cdot y \\
& \frac{\partial \psi}{\partial y}=\frac{\partial\left(\Omega_{0}+\Omega_{1} \cdot y\right)}{\partial y}=\frac{\partial \Omega_{0}}{\partial y}+\frac{\partial\left(\Omega_{1} \cdot y\right)}{\partial y}=\frac{\partial \Omega_{0}}{\partial y}+\left(\frac{\partial \Omega_{1}}{\partial y} \cdot y+\Omega_{1} \cdot \frac{\partial x}{\partial x}\right)=\frac{\partial \Omega_{0}}{\partial y}+\frac{\partial \Omega_{1}}{\partial y} \cdot y+\Omega_{1}
\end{aligned}
$$

Antes da realização das segundas derivadas para a obtenção das funções de Airy, são realizadas substituições com o intuito de simplificar os cálculos. Sendo as seguintes relações utilizadas neste procedimento:

$$
\Psi=\frac{\partial \Omega_{1}}{\partial x} \quad \phi=\frac{\partial \Omega_{1}}{\partial y} \quad \chi=\frac{\partial \Omega_{0}}{\partial x} \quad \varphi=\frac{\partial \Omega_{0}}{\partial y}
$$


Partindo então para as substituições de (C.3) em (C.2) além das segundas derivadas obtêm-se:

$$
\begin{aligned}
& \sigma_{x}=\frac{\partial^{2} \psi}{\partial y^{2}}=\frac{\partial}{\partial y}\left(\varphi+\phi \cdot y+\Omega_{1}\right)=\frac{\partial \varphi}{\partial y}+\frac{\partial(\phi \cdot y)}{\partial y}+\phi=\frac{\partial \varphi}{\partial y}+\left(\frac{\partial \phi}{\partial y} y+\phi \frac{\partial y}{\partial y}\right)+\phi=2 \phi+\frac{\partial \phi}{\partial y} y+\frac{\partial \varphi}{\partial y} \\
& \sigma_{y}=\frac{\partial^{2} \psi}{\partial x^{2}}=\frac{\partial}{\partial x}\left(\frac{\partial \Omega_{0}}{\partial x}+\frac{\partial \Omega_{1}}{\partial x} \cdot y\right)=\frac{\partial \chi}{\partial x}+\frac{\partial \Psi}{\partial x} y \\
& \tau_{x y}=-\frac{\partial^{2} \psi}{\partial x \partial y}=-\frac{\partial}{\partial y}\left(\frac{\partial \Omega_{0}}{\partial x}+\frac{\partial \Omega_{1}}{\partial x} \cdot y\right)=-\frac{\partial \chi}{\partial y}-\frac{\partial(\Psi \cdot y)}{\partial y}=-\frac{\partial \chi}{\partial y}-\frac{\partial \Psi}{\partial y} y-\Psi
\end{aligned}
$$

Tendo como base a condição de que este conjunto de funções harmônicas deve satisfazer à equação de Laplace e utilizando as relações de (C.3) obtêm-se que:

$$
\begin{gathered}
\nabla^{2}=\left(\frac{\partial^{2}}{\partial x^{2}}+\frac{\partial^{2}}{\partial y^{2}}\right) \Omega_{1}=0 \Rightarrow \frac{\partial^{2} \Omega_{1}}{\partial x^{2}}+\frac{\partial^{2} \Omega_{1}}{\partial y^{2}}=0 \Rightarrow \frac{\partial^{2} \Omega_{1}}{\partial x^{2}}=-\frac{\partial^{2} \Omega_{1}}{\partial y^{2}} \\
\Rightarrow \frac{\partial}{\partial x}\left(\frac{\partial \Omega_{1}}{\partial x}\right)=-\frac{\partial}{\partial y}\left(\frac{\partial \Omega_{1}}{\partial y}\right) \Rightarrow \frac{\partial \Psi}{\partial x}=-\frac{\partial \phi}{\partial y} e \frac{\partial \phi}{\partial x}=\frac{\partial \Psi}{\partial y} \\
\nabla^{2}=\left(\frac{\partial^{2}}{\partial x^{2}}+\frac{\partial^{2}}{\partial y^{2}}\right) \Omega_{0}=0 \Rightarrow \frac{\partial^{2} \Omega_{0}}{\partial x^{2}}+\frac{\partial^{2} \Omega_{0}}{\partial y^{2}}=0 \Rightarrow \frac{\partial^{2} \Omega_{0}}{\partial x^{2}}=-\frac{\partial^{2} \Omega_{0}}{\partial y^{2}} \\
\Rightarrow \frac{\partial}{\partial x}\left(\frac{\partial \Omega_{0}}{\partial x}\right)=-\frac{\partial}{\partial y}\left(\frac{\partial \Omega_{0}}{\partial y}\right) \Rightarrow \frac{\partial \varphi}{\partial x}=-\frac{\partial \chi}{\partial y}
\end{gathered}
$$

Utilizando algumas das relações expressas em (C.5) reescreve-se as funções (C.4) da seguinte maneira:

$$
\begin{aligned}
& \sigma_{x}=\frac{\partial^{2} \psi}{\partial y^{2}}=2 \phi+\frac{\partial \phi}{\partial y} y+\frac{\partial \varphi}{\partial y}=2 \phi+y \frac{\partial \phi}{\partial y}-\frac{\partial \chi}{\partial x} \\
& \sigma_{y}=\frac{\partial^{2} \psi}{\partial x^{2}}=y \frac{\partial \Psi}{\partial x}+\frac{\partial \chi}{\partial x}=-y \frac{\partial \phi}{\partial y}+\frac{\partial \chi}{\partial x} \\
& \tau_{x y}=-\frac{\partial^{2} \psi}{\partial x \partial y}=-\Psi-y \frac{\partial \Psi}{\partial y}-\frac{\partial \chi}{\partial y}=-\Psi-y \frac{\partial \phi}{\partial x}-\frac{\partial \chi}{\partial y}
\end{aligned}
$$


Um ponto importante a se destacar é que as equações expressas por (C.6) são genéricas em termos de comportamento da chapa. Uma vez que se deseja a obtenção das equações que descrevem o modo I de abertura, para isto, basta-se apenas a imposição das devidas condições de contorno. No caso, impõe-se que as tensões de cisalhamento na face da fissura $(y=0)$ sejam nulas:

$$
\begin{gathered}
\tau_{x y}=-\Psi-y \frac{\partial \phi}{\partial x}-\frac{\partial \chi}{\partial y}=-\Psi-(0) \frac{\partial \phi}{\partial x}-\frac{\partial \chi}{\partial y}=0 \quad \Rightarrow \quad \Psi=-\frac{\partial \chi}{\partial y} \\
\tau_{x y}=-\Psi-y \frac{\partial \phi}{\partial x}-\frac{\partial \chi}{\partial y}=-\left(\frac{\partial \chi}{\partial y}\right)-y \frac{\partial \phi}{\partial x}-\frac{\partial \chi}{\partial x} \Rightarrow \tau_{x y}=-y \frac{\partial \phi}{\partial x}
\end{gathered}
$$

Sendo assim, a mesma relação obtida em (C.7) é utilizada para a definição das tensões normais, sendo esta relacionada juntamente com aquelas obtidas em (C.5) proporcionando:

$$
\begin{aligned}
\frac{\partial \Psi}{\partial x}=-\frac{\partial \phi}{\partial y} \Leftrightarrow \Psi=-\frac{\partial \chi}{\partial y} & \Rightarrow \frac{\partial \Psi}{\partial x}=-\frac{\partial \phi}{\partial y} \Rightarrow \frac{\partial}{\partial x}\left(-\frac{\partial \chi}{\partial y}\right)=-\frac{\partial \phi}{\partial y} \Rightarrow \\
& \Rightarrow-\frac{\partial}{\partial y}\left(\frac{\partial \chi}{\partial x}\right)=-\frac{\partial}{\partial x}(\phi) \Rightarrow \phi=\frac{\partial \chi}{\partial x}
\end{aligned}
$$

Inserindo a equação (C.9) nas duas primeiras equações de (C.6) temos que:

$$
\begin{gathered}
\sigma_{x}=2 \phi+y \frac{\partial \phi}{\partial y}-\frac{\partial \chi}{\partial x}=2 \phi+y \frac{\partial \phi}{\partial y}-(\phi)=\phi+y \frac{\partial \phi}{\partial y} \\
\sigma_{y}=-y \frac{\partial \phi}{\partial y}+\frac{\partial \chi}{\partial x}=-y \frac{\partial \phi}{\partial y}+(\phi)=\phi-y \frac{\partial \phi}{\partial y}
\end{gathered}
$$


Utilizando uma única relação de (C.5) ficam definidas as equações das tensões para o modo I de abertura:

$$
\begin{aligned}
\sigma_{x}=\phi+y \frac{\partial \phi}{\partial y}=\phi+y\left(-\frac{\partial \Psi}{\partial x}\right) & \Rightarrow \sigma_{x}=\phi-y \frac{\partial \Psi}{\partial x} \\
\sigma_{y}=\phi-y \frac{\partial \phi}{\partial y}=\phi-y\left(-\frac{\partial \Psi}{\partial x}\right) & \Rightarrow \quad \sigma_{y}=\phi+y \frac{\partial \Psi}{\partial x}
\end{aligned}
$$

$$
\tau_{x y}=-y \frac{\partial \phi}{\partial x}
$$

Para a definição das equações das tensões referentes ao modo II de abertura, aplicamse as devidas condições de contorno às equações (C.6). Uma delas refere-se à tensão de tração nula na face da fissura $(y=0)$ :

$$
\sigma_{y}=y \frac{\partial \Psi}{\partial x}+\frac{\partial \chi}{\partial x}=-(0) \frac{\partial \phi}{\partial y}+\frac{\partial \chi}{\partial x}=0 \quad \Rightarrow \quad \frac{\partial \chi}{\partial x}=0
$$

Como conseqüência da relação obtida anteriormente (C.12), juntamente com as relações escritas em (C.5), tem-se também que:

$$
\begin{gathered}
\frac{\partial \chi}{\partial x}=-\frac{\partial \varphi}{\partial y}=0 \Leftrightarrow \frac{\partial \chi}{\partial y}=\frac{\partial \varphi}{\partial x} \Rightarrow \text { isolando } \partial \varphi \Rightarrow \\
\partial \varphi=-\frac{\partial \chi}{\partial x} \partial y \Leftrightarrow \partial \varphi=\frac{\partial \chi}{\partial y} \partial x \Rightarrow \text { igualando } \partial \varphi \Rightarrow \\
\Rightarrow-\frac{\partial \chi}{\partial x} \partial y=\frac{\partial \chi}{\partial y} \partial x \Rightarrow-\frac{\partial \chi}{\partial x} \frac{\partial y}{\partial x}=\frac{\partial \chi}{\partial y} \Rightarrow \frac{\partial \chi}{\partial y}=-\frac{\partial \chi}{\partial x} \frac{\partial y}{\partial x}=-(0) \frac{\partial y}{\partial x}=0 \Rightarrow \frac{\partial \chi}{\partial y}=0
\end{gathered}
$$

Impondo as condições (C.12) e (C.13) em (C.6) e utilizando algumas relações de (C.5) tem-se as equações que descrevem o comportamento das tensões atuando no modo II de abertura: 


$$
\begin{aligned}
& \sigma_{x}=2 \phi+y \frac{\partial \phi}{\partial y}-\frac{\partial \not \partial}{\partial x}=2 \phi+y\left(-\frac{\partial \Psi}{\partial x}\right)=2 \phi-y \frac{\partial \Psi}{\partial x} \\
& \sigma_{y}=-y \frac{\partial \phi}{\partial y}+\frac{\partial \chi}{\partial x}=-y\left(-\frac{\partial \Psi}{\partial x}\right)+\frac{\partial \not \partial}{\partial x}=y \frac{\partial \Psi}{\partial x} \\
& \tau_{x y}=-\Psi-y \frac{\partial \phi}{\partial x}-\frac{\partial \not \partial}{\partial y}=-\Psi-y \frac{\partial \phi}{\partial x}
\end{aligned}
$$

Deduzidas as equações de tensões (em função das equações harmônicas), sintetiza-se então no quadro a seguir estas equações (representativas dos modos I e II de abertura) além daquelas deduzidas por Westergaard:

Tabela C.1 - Comparativo entre as Funções-Tensão de Westergaard e as Tensões

\begin{tabular}{|c|c|c|}
\hline $\begin{array}{c}\text { Modo de } \\
\text { Abertura }\end{array}$ & $\begin{array}{c}\text { Funções Deduzidas } \\
\text { das Equações Harmônicas }\end{array}$ & $\begin{array}{c}\text { Funções Complexas de Tensão } \\
\text { Finais de Westergaard }\end{array}$ \\
\hline \multirow{3}{*}{ I } & $\sigma_{x}=\phi-y \frac{\partial \Psi}{\partial x}$ & $\sigma_{x}=\operatorname{Re} Z_{I}-y \cdot \operatorname{Im} Z_{I}^{\prime}$ \\
\cline { 2 - 3 } & $\sigma_{y}=\phi+y \frac{\partial \Psi}{\partial x}$ & $\sigma_{y}=\operatorname{Re} Z_{I}+y \cdot \operatorname{Im} Z_{I}^{\prime}$ \\
\hline \multirow{3}{*}{ II } & $\tau_{x y}=-y \frac{\partial \phi}{\partial x}$ & $\tau_{x y}=-y \cdot \operatorname{Re} Z_{I}^{\prime}$ \\
\cline { 2 - 3 } & $\sigma_{x}=2 \phi-y \frac{\partial \Psi}{\partial x}$ & $\sigma_{x}=2 \cdot \operatorname{Re} Z_{I I}-y \cdot \operatorname{Im} Z_{I I}^{\prime}$ \\
\cline { 2 - 3 } & $\sigma_{y}=y \frac{\partial \Psi}{\partial x}$ & $\sigma_{y}=y \cdot \operatorname{Im} Z_{I I}^{\prime}$ \\
\cline { 2 - 3 } & $\tau_{x y}=-\Psi-y \frac{\partial \phi}{\partial x}$ & $\tau_{x y}=-\operatorname{Im} Z_{I I}-y \cdot \operatorname{Re} Z_{I I}^{\prime}$ \\
\hline
\end{tabular}

Comparando-se as equações da primeira com a segunda coluna, observa-se uma semelhança no que se refere às ordens de derivadas, os termos multiplicativos e os sinais, onde podem-se dizer que, em virtude das seguintes semelhanças:

$$
\phi=\operatorname{Re} Z \quad \frac{\partial \phi}{\partial x}=\operatorname{Re} Z^{\prime} \quad \Psi=\operatorname{Im} Z \quad \frac{\partial \Psi}{\partial x}=\operatorname{Im} Z^{\prime}
$$


as equações desenvolvidas por Westergaard expressas por (2.18) e (2.19) são válidas uma vez que o seu desenvolvimento gera funções de tensão com as mesmas características daquelas desenvolvidas através de um conjunto de funções harmônicas e suas derivadas apresentadas neste apêndice. A demonstração é apresentada a seguir, sendo realizadas duas integrais da função $\sigma_{y}$ (de Airy ) em relação à variável $x$, tanto para o modo I quanto para o modo II de abertura, para a obtenção de $\psi_{I}$ e $\psi_{I I}$. Sendo assim, realizando a primeira integral e utilizando as relações de (C.15):

$\sigma_{y}=\frac{\partial^{2} \psi_{I}}{\partial x^{2}}=\frac{\partial}{\partial x}\left(\frac{\partial \psi_{I}}{\partial x}\right)=\phi+y \frac{\partial \Psi}{\partial x}=\operatorname{Re} Z_{I}+y \operatorname{Im} Z_{I}^{\prime} \quad \Rightarrow \quad \frac{\partial \psi_{I}}{\partial x}=\int \operatorname{Re} Z_{I} \partial x+\int y \operatorname{Im} Z_{I}^{\prime} \partial x$

Das condições de Cauchy-Riemann (ver Apêndice B) tem-se que:

$$
\begin{aligned}
& \operatorname{Re} Z_{I}^{\prime}=\frac{\partial \operatorname{Re} Z_{I}}{\partial z}=\frac{\partial \operatorname{Re} Z_{I}}{\partial x} \Rightarrow \operatorname{Re} Z_{I}=\int \frac{\partial \operatorname{Re} Z_{I}}{\partial x} \partial z \\
& \operatorname{Re} Z_{I}=\int \frac{\partial \operatorname{Re} Z_{I}}{\partial x} \partial z \Rightarrow \int \operatorname{Re} Z_{I} \partial z=\iint \frac{\partial \operatorname{Re} Z_{I}}{\partial x} \partial z \partial z \Rightarrow \operatorname{Re} \tilde{Z}_{I}=\iint \frac{\partial \operatorname{Re} Z_{I} \partial z \partial z}{\partial x}
\end{aligned}
$$

$\operatorname{Im} Z_{I}^{\prime}=\frac{\partial \operatorname{Im} Z_{I}}{\partial x}$

$\operatorname{Im} Z_{I}^{\prime}=\frac{\partial \operatorname{Im} Z_{I}}{\partial x} \Rightarrow \frac{\partial \operatorname{Im} Z_{I}}{\partial z}=\frac{\partial \operatorname{Im} Z_{I}}{\partial x} \Rightarrow \operatorname{Im} Z_{I}=\int \frac{\partial \operatorname{Im} Z_{I}}{\partial x} \partial z$ 
Inserindo as relações (C.17a) e (C.17c) em (C.16) tem-se:

$$
\begin{gathered}
\frac{\partial \psi_{I}}{\partial x}=\iint \frac{\operatorname{Re} Z_{I}}{\partial x} \partial x \partial z+\int y \frac{\partial \operatorname{Im} Z_{I}}{\partial x} \partial x=\iint \partial \operatorname{Re} Z_{I} \partial z+\int y \partial \operatorname{Im} Z_{I} \Rightarrow \\
\Rightarrow \frac{\partial \psi_{I}}{\partial x}=\int \operatorname{Re} Z_{I} \partial z+y \int \partial \operatorname{Im} Z_{I} \Rightarrow \frac{\partial \psi_{I}}{\partial x}=\operatorname{Re} \tilde{Z}_{I}+y \operatorname{Im} Z_{I}
\end{gathered}
$$

Inserindo as relações (C.17b) e (C.17d) em (C.18) e realizando a segunda integral em relação a $\boldsymbol{x}$ obtêm-se a primeira das duas funções de tensão de Westergaard (modo I de abertura):

$$
\begin{aligned}
\psi_{I} & =\int \operatorname{Re} \tilde{Z}_{I} \partial x+\int y \operatorname{Im} Z_{I} \partial x=\iiint \frac{\operatorname{Re} \tilde{Z}_{I}}{\partial x} \partial x \partial z \partial z+\iint y \frac{\partial \operatorname{Im} Z_{I}}{\partial x} \partial x \partial z \Rightarrow \\
& \Rightarrow \psi_{I}=\iint \operatorname{Re} Z_{I} \partial z \partial z+y \int \operatorname{Im} Z_{I} \partial z \Rightarrow \psi_{I}=\operatorname{Re} \tilde{\tilde{Z}}_{I}+y \operatorname{Im} \tilde{Z}_{I}
\end{aligned}
$$

Para a determinação da segunda equação de tensão de Westergaard parte-se da função $\sigma_{y}$ (modo II de abertura) realizando a sua primeira integral e em seguida utilizam-se as relações expressas em (C.15):

$$
\sigma_{y}=\frac{\partial^{2} \psi_{I I}}{\partial x^{2}}=\frac{\partial}{\partial x}\left(\frac{\partial \psi_{I I}}{\partial x}\right)=y \frac{\partial \Psi}{\partial x}=y \cdot \operatorname{Im} Z_{I I}^{\prime}=\quad \Rightarrow \quad \frac{\partial \psi_{I I}}{\partial x}=\int y \operatorname{Im} Z_{I I}^{\prime} \partial x
$$

Das condições de Cauchy-Riemann (ver Apêndice B) tem-se que:

$$
\begin{aligned}
& \operatorname{Im} Z_{I I}^{\prime}=\frac{\partial \operatorname{Im} Z_{I I}}{\partial x} \\
& \operatorname{Im} Z_{I I}^{\prime}=\frac{\partial \operatorname{Im} Z_{I I}}{\partial x} \Rightarrow \frac{\partial \operatorname{Im} Z_{I I}}{\partial z}=\frac{\partial \operatorname{Im} Z_{I I}}{\partial x} \Rightarrow \operatorname{Im} Z_{I I}=\int \frac{\partial \operatorname{Im} Z_{I I} \partial z}{\partial x}
\end{aligned}
$$


Retomando (C.20) para realizar a segunda integral e utilizando-se as relações de (C.21) obtêm-se a função tensão de Westergaard para o modo II de abertura:

$$
\begin{gathered}
\frac{\partial \psi_{I I}}{\partial x}=\int y \operatorname{Im} Z_{I I}^{\prime} \partial x \Rightarrow \psi_{I I}=\iint y \operatorname{Im} Z_{I I}^{\prime} \partial x \partial x=\iint y \frac{\partial \operatorname{Im} Z_{I I}}{\partial x} \partial x \partial x=\int y \operatorname{Im} Z_{I I} \partial x \\
\Rightarrow \psi_{I I}=\iint y \frac{\partial \operatorname{Im} Z_{I I}}{\partial x} \partial z \partial x=y \iint \partial \operatorname{Im} Z_{I I} \partial z=y \int \operatorname{Im} Z_{I I} \partial z \Rightarrow \psi_{I I}=y \operatorname{Im} \tilde{Z}_{I I}
\end{gathered}
$$




\section{Apêndice D}

Campo de Deslocamentos em Função de Z Considerando EPT e EPD 
Para a obtenção das expressões que relacionam deslocamentos e tensões, para o modo I de abertura e EPT ( $\left.\sigma_{Z}=0\right)$, necessário se faz inserir as equações (2.26) e (2.27) em (2.39) e (2.40):

$$
\begin{aligned}
& u=\frac{1}{E}\left[\int \sigma_{x} \partial x-v \int\left(\sigma_{y}+\phi_{z}^{\prime}\right) d x\right]=\frac{1}{E}\left[\int \sigma_{x} d x-v \int \sigma_{y} d x\right] \Rightarrow \\
& \Rightarrow=\frac{1}{E}\left[\int\left(\operatorname{Re} Z_{I}-y \cdot \operatorname{Im} Z_{I}^{\prime}\right) d x-v \int\left(\operatorname{Re} Z_{I}+y \cdot \operatorname{Im} Z_{I}^{\prime}\right) d x\right] \Rightarrow \\
& \Rightarrow(2 G) u=(2 G) \frac{1}{E}\left[\operatorname{Re} \tilde{Z}_{I}-y \cdot \operatorname{Im} Z_{I}-v \cdot \operatorname{Re} \tilde{Z}_{I}-y v \cdot \operatorname{Im} Z_{I}\right] \Rightarrow 2 G u=\frac{(1-v)}{(v+1)} \cdot \operatorname{Re} \tilde{Z}_{I}-y \cdot \operatorname{Im} Z_{I}
\end{aligned}
$$

$$
\begin{aligned}
& v=\frac{1}{E}\left[\int \sigma_{y} \partial y-v \int\left(\sigma_{x}+\phi_{z}^{\prime}\right) d y\right]=\frac{1}{E}\left[\int \sigma_{y} d y-v \int \sigma_{x} d y\right] \Rightarrow \\
& \Rightarrow v=\frac{1}{E}\left[\int\left(\operatorname{Re} Z_{I}+y \cdot \operatorname{Im} Z_{I}^{\prime}\right) d y-v \int\left(\operatorname{Re} Z_{I}-y \cdot \operatorname{Im} Z_{I}^{\prime}\right) d y\right] \Rightarrow \\
& \therefore \text { (int egração por partes) } \Rightarrow \int y \cdot \operatorname{Im} Z_{I}^{\prime} d y \Rightarrow \\
& \Rightarrow \varpi=y, \frac{d \Phi}{d y}=1, d \varsigma=\operatorname{Im} Z_{I}^{\prime} d y, \quad \varsigma=\int \operatorname{Im} Z_{I}^{\prime} d y=-\operatorname{Re} Z_{I} \\
& \Rightarrow \int y \cdot \operatorname{Im} Z_{I}^{\prime} d y=\int \varpi d \varsigma=\varpi \cdot \varsigma-\int \varsigma d \varpi=y \cdot\left(-\operatorname{Re} Z_{I}\right)-\int\left(-\operatorname{Re} Z_{I}\right) d y=-y \cdot \operatorname{Re} Z_{I}+\operatorname{Im} \tilde{Z}_{I} \\
& \Rightarrow 2 G v=\frac{1}{(v+1)} \cdot\left[-y \cdot \operatorname{Re} Z_{I}(1+v)+2 \operatorname{Im} \tilde{Z}_{I}\right] \Rightarrow 2 G v=\frac{2}{(1+v)} \cdot \operatorname{Im} \tilde{Z}_{I}-\operatorname{Re} Z_{I} \cdot y
\end{aligned}
$$

Considerando-se estado plano de deformação (EPD) onde temos $\sigma_{z}=\left(\sigma_{x}+\sigma_{y}\right) / 2$, as equações de deslocamento para o modo I de abertura são:

$$
\begin{aligned}
u & =\frac{1}{E}\left[\int \sigma_{x} d x-v \int\left(\sigma_{y}+\sigma_{z}\right) d x\right]=\frac{\left(1-v^{2}\right)}{E}\left[\int \sigma_{x} \partial x-\frac{v}{(1-v)} \int \sigma_{y} d x\right] \Rightarrow \\
& \Rightarrow 2 G u=\frac{2 G \cdot\left(1-v^{2}\right)}{E}\left[\int\left(\operatorname{Re} \tilde{Z}_{I}-y \cdot \operatorname{Im} Z_{I}\right) d x-\frac{v}{(1-v)} \int\left(\operatorname{Re} \tilde{Z}_{I}+y \cdot \operatorname{Im} Z_{I}\right) d x\right] \Rightarrow \\
& \Rightarrow 2 G u=\frac{2 G \cdot(1+v)}{E}\left[(1-2 v) \cdot \operatorname{Re} \tilde{Z}_{I}-y \cdot \operatorname{Im} Z_{I}\right] \Rightarrow 2 G u=(1-2 v) \cdot \operatorname{Re} \tilde{Z}_{I}-y \cdot \operatorname{Im} Z_{I}
\end{aligned}
$$




$$
\begin{aligned}
& v=\frac{1}{E}\left[\int \sigma_{y} d y-v \int\left(\sigma_{x}+\sigma_{z}\right) d y\right]=\frac{\left(1-v^{2}\right)}{E}\left[\int \sigma_{y} d y-\frac{v}{(1-v)} \int \sigma_{x} d y\right] \Rightarrow \\
& v=\frac{\left(1-v^{2}\right)}{E}\left[\int\left(\operatorname{Re} Z_{I}+y \cdot \operatorname{Im} Z_{I}^{\prime}\right) d y-\frac{v}{(1-v)} \int\left(\operatorname{Re} Z_{I}-y \cdot \operatorname{Im} Z_{I}^{\prime}\right) d y\right] \Rightarrow \\
& v=\frac{\left(1-v^{2}\right)}{E}\left\{\operatorname{Im} \tilde{Z}_{I}+\left(-y \cdot \operatorname{Re} Z_{I}+\operatorname{Im} \tilde{Z}_{I}\right)-\frac{v}{(1-v)}\left[\operatorname{Im} \tilde{Z}_{I}-\left(-y \cdot \operatorname{Re} Z_{I}+\operatorname{Im} \tilde{Z}_{I}\right]\right\} \Rightarrow\right. \\
& \Rightarrow 2 G v=2 G \cdot(1-v) \cdot\left[2 \operatorname{Im} \tilde{Z}_{I}-\frac{\operatorname{Re} Z_{I} \cdot y}{(1-v)}\right] \Rightarrow 2 G v=2 \cdot(1-v) \cdot \operatorname{Im} \tilde{Z}_{I}-\operatorname{Re} Z_{I} \cdot y
\end{aligned}
$$

Para o modo II de abertura, inserindo as equações (2.29), (2.30) e (2.31) em (2.39) e (2.40), tem-se as seguintes equações de deslocamentos para EPT ( $\left.\sigma_{Z}=0\right)$ :

$$
\begin{aligned}
& 2 G u=\frac{2}{(v+1)} \cdot \operatorname{Re} \tilde{Z}_{I I}+y \cdot \operatorname{Im} Z_{I I} \\
& 2 G v=\frac{(1-v)}{(1+v)} \cdot \operatorname{Im} \tilde{Z}_{I I}-y \cdot \operatorname{Re} Z_{I I}
\end{aligned}
$$

Considerando ainda o modo II de abertura, têm-se as seguintes expressões de deslocamentos para $\operatorname{EPD}\left(\sigma_{z}=\left(\sigma_{x}+\sigma_{y}\right) / 2\right)$ :

$2 G u=2(1-v) \cdot \operatorname{Re} \tilde{Z}_{I I}-y \cdot \operatorname{Im} Z_{I I}$

$$
2 G v=(1-2 v) \cdot \operatorname{Im} \tilde{Z}_{I I}-y \cdot \operatorname{Re} Z_{I I}
$$


Apêndice $E$

Campo de Tensões em Função de r e $\theta$ Modos I e II de Abertura 
Para a obtenção das equações que definem o campo de tensão próximo à ponta da fissura, em função de $\boldsymbol{r}$ e $\boldsymbol{\theta}$, consideram-se primeiramente estas sete relações:

$e^{i \theta}=\sum_{k=0}^{\infty} \frac{(i \theta)^{k}}{k !}=\sum_{k=0}^{\infty} \frac{\theta^{2 k}}{(2 k) !}+i \cdot \sum_{k=0}^{\infty} \frac{\theta^{2 k+1}}{(2 k+1) !}=\cos \theta+i \cdot \operatorname{sen} \theta$

$\xi=a+b \cdot i=(r \cdot \cos \theta)+(r \cdot \operatorname{sen} \theta) \cdot i=r \cdot(\cos \theta+i \cdot \operatorname{sen} \theta)=r \cdot e^{i \theta}$

(onde " $a$ " $\mathrm{e} " ~ b$ " representam as coordenadas dos eixos real e imaginário, respectivamente)

$\cos \theta=\frac{e^{i \theta}+e^{-i \theta}}{2}$

$\operatorname{sen} \theta=\frac{e^{i \theta}-e^{-i \theta}}{2 i}$

$\cos \theta=\frac{e^{i \theta}+e^{-i \theta}}{2} \Rightarrow e^{-i \theta}=2 \cdot \cos \theta-e^{i \theta}=2 \cdot \cos \theta-(\cos \theta+i \cdot \operatorname{sen} \theta) \Rightarrow e^{-i \cdot \theta}=\cos \theta-i \cdot \operatorname{sen} \theta$

$e^{-i \cdot \frac{3 \theta}{2}}=\cos \left(\frac{3 \theta}{2}\right)-i \cdot \operatorname{sen}\left(\frac{3 \theta}{2}\right) \quad$ (análoga à anterior)

$\operatorname{sen} \theta=2 \cdot \operatorname{sen}\left(\frac{\theta}{2}\right) \cdot \cos \left(\frac{\theta}{2}\right)$

Utilizando-se a relação (E.2) na equação (2.44) obtêm-se a equação de tensão em função das variáveis $\boldsymbol{r}$ e $\boldsymbol{\theta}$ :

$$
Z_{I}=\frac{K_{I}}{\sqrt{2 \pi}} \cdot \xi^{-1 / 2}=\frac{K_{I}}{\sqrt{2 \pi}} \cdot\left(r e^{i \theta}\right)^{-1 / 2}=\frac{K_{I}}{\sqrt{2 \pi r}} \cdot e^{-i \frac{\theta}{2}} \Rightarrow Z_{I}=\frac{K_{I}}{\sqrt{2 \pi r}} \cdot\left[\cos \left(\frac{\theta}{2}\right)-i \cdot \operatorname{sen}\left(\frac{\theta}{2}\right)\right]
$$


Como as equações (2.26) até (2.31) que definem as tensões $\sigma_{x}, \sigma_{y}, \tau_{x y}$ encontramse em função de $Z_{I}$ e $Z_{I}^{\prime}$, importante se faz obter a primeira derivada da equação (E.8), conforme mostrado abaixo:

$$
\begin{aligned}
Z_{I}^{\prime} & =\frac{\partial Z_{I}}{\partial \xi}=\frac{\partial}{\partial \xi}\left(\frac{K_{I}}{\sqrt{2 \pi}} \cdot \xi^{-1 / 2}\right)=\left(-\frac{1}{2}\right) \cdot \frac{K_{I}}{\sqrt{2 \pi}} \cdot \xi^{\frac{-1}{2}-1}=\frac{-K_{I}}{2 \sqrt{2 \pi}} \cdot \xi^{\frac{-3}{2}}=\frac{-K_{I}}{2 \sqrt{2 \pi}} \cdot\left(r \cdot e^{i \theta}\right)^{\frac{-3}{2}} \Rightarrow \\
& \Rightarrow \frac{\partial Z_{I}}{\partial \xi}=\frac{-K_{I}}{2 r^{3 / 2} \sqrt{2 \pi}} \cdot e^{\frac{-3 i \theta}{2}}=\frac{-K_{I}}{2 r^{3 / 2} \sqrt{2 \pi}} \cdot e^{\frac{-3 i \theta}{2}} \Rightarrow \\
\Rightarrow & \frac{\partial Z_{I}}{\partial \xi}=Z_{I}^{\prime}=\frac{-K_{I}}{2 r^{3 / 2} \sqrt{2 \pi}} \cdot\left[\cos \left(\frac{3 \theta}{2}\right)-i \cdot \operatorname{sen}\left(\frac{3 \theta}{2}\right)\right]
\end{aligned}
$$

Sendo as funções $Z_{I}$ e $Z_{I}^{\prime}$ constituídas de partes reais e imaginárias, tem-se que $Z_{I}=\operatorname{Re} Z_{I}+i \cdot \operatorname{Im} Z_{I} \quad$ e $\quad Z_{I}^{\prime}=\operatorname{Re} Z_{I}^{\prime}+i \cdot \operatorname{Im} Z_{I}^{\prime}$. Desta forma, definem-se abaixo as expressões dos termos reais e imaginários de (E.8) e (E.9):

$\operatorname{Re} Z_{I}=\frac{K_{I}}{\sqrt{2 \pi r}} \cdot \cos \left(\frac{\theta}{2}\right)$

$\operatorname{Im} Z_{I}=\frac{K_{I}}{\sqrt{2 \pi r}} \cdot\left[-\operatorname{sen}\left(\frac{\theta}{2}\right)\right]$

$\operatorname{Re} Z_{I}^{\prime}=\frac{-K_{I}}{2 r^{3 / 2} \sqrt{2 \pi}} \cdot \cos \left(\frac{3 \theta}{2}\right)=\frac{-K_{I}}{2 r \sqrt{2 \pi r}} \cdot \cos \left(\frac{3 \theta}{2}\right)$

$\operatorname{Im} Z_{I}^{\prime}=\frac{-K_{I}}{2 r^{3 / 2} \sqrt{2 \pi}} \cdot\left[-\operatorname{sen}\left(\frac{3 \theta}{2}\right)\right]=\frac{K_{I}}{2 r \sqrt{2 \pi r}} \cdot \operatorname{sen}\left(\frac{3 \theta}{2}\right)$ 
Inserindo as expressões (E.10), (E.11), (E.12) e (E.13) em (2.26), (2.27), (2.28) e utilizando a relação abaixo:

$y=r \cdot \operatorname{sen} \theta=r \cdot\left[2 \operatorname{sen}\left(\frac{\theta}{2}\right) \cdot \cos \left(\frac{\theta}{2}\right)\right]$

têm-se as expressões para a obtenção dos valores das tensões próximas à região da ponta da fissura, considerando-se o modo I de abertura:

$$
\begin{aligned}
& \sigma_{x}=\frac{K_{I}}{\sqrt{2 \pi r}} \cdot \cos \left(\frac{\theta}{2}\right) \cdot\left[1-\operatorname{sen}\left(\frac{\theta}{2}\right) \cdot \operatorname{sen}\left(\frac{3 \theta}{2}\right)\right] \\
& \sigma_{y}=\frac{K_{I}}{\sqrt{2 \pi r}} \cdot \cos \left(\frac{\theta}{2}\right) \cdot\left[1+\operatorname{sen}\left(\frac{\theta}{2}\right) \cdot \operatorname{sen}\left(\frac{3 \theta}{2}\right)\right] \\
& \tau_{x y}=\frac{K_{I}}{\sqrt{2 \pi r}} \cdot \cos \left(\frac{\theta}{2}\right) \cdot\left[\operatorname{sen}\left(\frac{\theta}{2}\right) \cdot \cos \left(\frac{3 \theta}{2}\right)\right]
\end{aligned}
$$

As expressões das tensões para o modo II de abertura são obtidas inserindo (E.10), (E.11), (E.12), (E.13) em (2.29), (2.30) e (2.31), e utilizando a relação (E.14):

$$
\begin{aligned}
& \sigma_{x}=\frac{-K_{I I}}{\sqrt{2 \pi r}} \cdot \operatorname{sen}\left(\frac{\theta}{2}\right) \cdot\left[2+\cos \left(\frac{\theta}{2}\right) \cdot \cos \left(\frac{3 \theta}{2}\right)\right] \\
& \sigma_{y}=\frac{K_{I I}}{\sqrt{2 \pi r}} \cdot \operatorname{sen}\left(\frac{\theta}{2}\right) \cdot \cos \left(\frac{\theta}{2}\right) \cdot \cos \left(\frac{3 \theta}{2}\right) \\
& \tau_{x y}=\frac{K_{I I}}{\sqrt{2 \pi r}} \cdot \cos \left(\frac{\theta}{2}\right) \cdot\left[1-\operatorname{sen}\left(\frac{\theta}{2}\right) \cdot \operatorname{sen}\left(\frac{3 \theta}{2}\right)\right]
\end{aligned}
$$


Para a determinação das equações que descrevem o campo de deslocamentos próximo à ponta da fissura, faz-se necessário inserir as equações (E.10), (E.11), (E.12), (E.13) e (E.14) nas expressões que definem o modo I de abertura (tabela 2.1). Com isto, adotando-se $\kappa=(3-4 v)$ para EPD e $\kappa=(3-v) /(1+v)$ para EPT, tem-se que:

$$
\begin{aligned}
& u=\frac{K_{I}}{2 G} \cdot \sqrt{\frac{r}{2 \pi}} \cdot \cos \left(\frac{\theta}{2}\right) \cdot(\kappa-\cos \theta) \\
& v=\frac{K_{I}}{2 G} \cdot \sqrt{\frac{r}{2 \pi}} \cdot \operatorname{sen}\left(\frac{\theta}{2}\right) \cdot(\kappa-\cos \theta)
\end{aligned}
$$

As equações de deslocamentos para o modo II de abertura são obtidas inserindo as equações (E.10), (E.11), (E.12), (E.13) e (E.14) nas expressões que definem o modo II de abertura (tabela 2.1). As mesmas considerações para EPD e EPT são adotadas de modo que:

$$
\begin{aligned}
& u=\frac{K_{I I}}{2 G} \cdot \sqrt{\frac{r}{2 \pi}} \cdot \operatorname{sen}\left(\frac{\theta}{2}\right) \cdot(2+\kappa+\cos \theta) \\
& v=\frac{K_{I I}}{2 G} \cdot \sqrt{\frac{r}{2 \pi}} \cdot \cos \left(\frac{\theta}{2}\right) \cdot(2-\kappa-\cos \theta)
\end{aligned}
$$




\section{Apêndice F}

Campo de

Deslocamentos e Tensões

- Modo III de Abertura 
Para o modo III de abertura temos as seguintes condições para tensões, deformações e deslocamentos:

$$
\begin{aligned}
& \sigma_{x}=\sigma_{y}=\sigma_{z}=\tau_{x y}=0 \\
& \varepsilon_{x}=\varepsilon_{y}=\varepsilon_{z}=\gamma_{x y}=0 \\
& u=v=0
\end{aligned}
$$

As relações entre deslocamento e deformação podem ser descritas como a seguir:

$$
\begin{aligned}
& \gamma_{x z}=\frac{\partial y}{\partial z}+\frac{\partial w}{\partial x}=\frac{\partial w}{\partial x} \\
& \gamma_{y z}=\frac{\partial y}{\partial z}+\frac{\partial w}{\partial y}=\frac{\partial w}{\partial y}
\end{aligned}
$$

Considerando-se a lei de Hooke:

$$
\begin{aligned}
& \tau_{x z}=G \cdot \gamma_{x z}=G \cdot\left(\frac{\partial w}{\partial x}\right) \\
& \tau_{y z}=G \cdot \gamma_{y z}=G \cdot\left(\frac{\partial w}{\partial y}\right)
\end{aligned}
$$

e utilizando uma função analítica que satisfaça a equação de Laplace $(\nabla w=0)$ :

$$
w=\frac{\operatorname{Im} Z_{I I I}}{G}
$$

pode-se então definir as tensões em relação à função analítica $Z_{I I I}$ :

$$
\begin{aligned}
& \tau_{x z}=G \cdot \frac{\partial w}{\partial x}=G \cdot \frac{\partial\left(\operatorname{Im} Z_{I I I} / G\right)}{\partial x}=\frac{\partial \operatorname{Im} Z_{I I I}}{\partial x}=\operatorname{Im} Z_{I I I}{ }^{\prime} \\
& \tau_{y z}=G \cdot \frac{\partial w}{\partial y}=G \cdot \frac{\partial\left(\operatorname{Im} Z_{I I I} / G\right)}{\partial y}=\frac{\partial \operatorname{Im} Z_{I I I}}{\partial y}=\operatorname{Re} Z_{I I I}{ }^{\prime}
\end{aligned}
$$


Desta forma, é possível utilizar a função tensão de Westergaard de maneira análoga à função (2.6):

$$
Z_{I I I}{ }^{\prime}=\frac{\tau \cdot z}{\sqrt{z^{2}-a^{2}}}=\frac{K_{I I I}}{\sqrt{2 \pi}} \cdot \xi^{-1 / 2}=\frac{K_{I I I}}{\sqrt{2 \pi r}} \cdot\left[\cos \left(\frac{\theta}{2}\right)-i \cdot \operatorname{sen}\left(\frac{\theta}{2}\right)\right] \quad \therefore \quad K_{I I I}=\tau \sqrt{\pi a}
$$

Como a equação de deslocamentos (F.4) depende de $Z_{I I I}$, esta pode ser obtida integrando a equação (F.6):

$$
\begin{aligned}
& Z_{I I I}=\int Z_{I I I}{ }^{\prime} \partial \xi=\int \frac{K_{I I I}}{\sqrt{2 \pi}} \cdot \xi^{-1 / 2} \partial \xi=\frac{K_{I I I}}{\sqrt{2 \pi}} \int \xi^{-1 / 2} \partial \xi=\frac{K_{I I I}}{\sqrt{2 \pi}} \cdot\left[\frac{\xi^{\frac{-1}{2}+1}}{\left(\frac{-1}{2}+1\right)} \cdot\right]=\frac{2 K_{I I I}}{\sqrt{2 \pi}} \cdot \xi^{1 / 2} \\
& \Rightarrow \quad=\frac{2 K_{I I I}}{\sqrt{2 \pi}} \cdot\left(r \cdot e^{i \theta}\right)^{1 / 2}=K_{I I I} \cdot \sqrt{\frac{2 r}{\pi}} \cdot e^{i \frac{\theta}{2}}=K_{I I I} \cdot \sqrt{\frac{2 r}{\pi}} \cdot\left[\cos \left(\frac{\theta}{2}\right)+i \cdot \operatorname{sen}\left(\frac{\theta}{2}\right)\right]
\end{aligned}
$$

Obtendo-se as partes reais e imaginárias das equações (F.6) e (F.7), tem-se que:

$$
\begin{aligned}
& \operatorname{Re} Z_{I I I}=K_{I I I} \cdot \sqrt{\frac{2 r}{\pi}} \cdot \cos \left(\frac{\theta}{2}\right) \\
& \operatorname{Im} Z_{I I I}=K_{I I I} \cdot \sqrt{\frac{2 r}{\pi}} \cdot \operatorname{sen}\left(\frac{\theta}{2}\right) \\
& \operatorname{Re} Z_{I I I}{ }^{\prime}=\frac{K_{I I I}}{\sqrt{2 \pi r}} \cdot \cos \left(\frac{\theta}{2}\right) \\
& \operatorname{Im} Z_{I I I}{ }^{\prime}=-\frac{K_{I I I}}{\sqrt{2 \pi r}} \cdot \operatorname{sen}\left(\frac{\theta}{2}\right)
\end{aligned}
$$


Utilizando as equações acima, pode-se então determinar as equações de tensão e deslocamento em função de $\boldsymbol{r}$ e $\boldsymbol{\theta}$ para modo III de abertura, inserindo-as em (F.4) e (F.5):

$$
\begin{aligned}
& \tau_{x z}=\operatorname{Im} Z_{I I I}^{\prime}=-\frac{K_{I I I}}{\sqrt{2 \pi r}} \cdot \operatorname{sen}\left(\frac{\theta}{2}\right) \\
& \tau_{y z}=\operatorname{Re} Z_{I I I}^{\prime}=\frac{K_{I I I}}{\sqrt{2 \pi r}} \cdot \cos \left(\frac{\theta}{2}\right) \\
& w=\frac{\operatorname{Im} Z_{I I I}}{G}=\frac{K_{I I I}}{G} \cdot \sqrt{\frac{2 r}{\pi}} \cdot \operatorname{sen}\left(\frac{\theta}{2}\right)
\end{aligned}
$$

